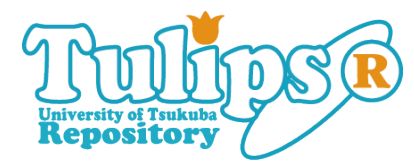

Properties of high-mass multijet events at the fermil ab proton-anti proton colli der

\begin{tabular}{|l|l|}
\hline 著者 & Asakawa Takashi \\
\hline 内容記述 & $\begin{array}{l}\text { Thesi s (Ph. D. i n Sci ence) - Uni ver si ty of } \\
\text { Tsukuba, (A) , no. 1598, 1996. 7. 25 }\end{array}$ \\
\hline year & 1996 \\
\hline URL & ht t p: //hdl . handl e. net /2241/00149165 \\
\hline
\end{tabular}




\section{Properties of High-Mass Multijet Events at the Fermilab Proton-Antiproton Collider}

Takashi ASAKAWA

A dissertation submitted to the Doctoral Program in Physics, the University of Tsukuba in partial fulfillment of the requirements for the degree of Doctor of Philosophy (Science)

February 1996 


\begin{abstract}
The properties of high-mass multijet events produced at the Fermilab proton-antiproton collider are compared with NJETS leading order QCD matrix element predictions, HERWIG QCD parton shower Monte Carlo predictions, and predictions from a model in which events are distributed uniformly over the available multibody phase-space. Multijet distributions corresponding to $(4 \mathrm{~N}-4)$ variables that span the $\mathrm{N}$-body parameter space are found to be well described by both the NJETS and HERWIG predictions for inclusive three-jet, four-jet, and five-jet events. The agreement between data, NJETS, and HERWIG suggests that $2 \rightarrow 2$ scattering plus gluon radiation provides a good first approximation to the full LO QCD matrix element for events with three, four, or even five jets in the final state. We see no clear evidence for any deviation from the predicted multijet distributions that might indicate new phenomena associated with the presence of many hard partons in the final state.
\end{abstract}




\section{Acknowledgements}

I want to thank Professor Kunitaka Kondo for his support throughout my graduate career. I was fortunate to have him as my advisor. He gave me the opportunity to go on the doctor course and then to work at CDF. He has always encouraged me since the beginning of my studies in 1990. I learned much from his serious attitude to physics research. I am also grateful for his careful reading of the text and his suggestions. I want to thank Professor Koji Takikawa for his continued encouragement and his support throughout my graduate career. Discussions on physics and my analysis with him were very precious.

I want to thank Steve Geer for his support and encouragement. He coordinated this analysis and provided me a lot of suggestions at crucial points. I learned a great deal from his precise way of thinking. I could not have finished this work on time without his continuous support. Many thanks go to Elizabeth Buckley Geer, Robert Harris and Brenna Flaugher, who gave me many useful suggestions and advice for this analysis. I am grateful to Andrea Castro and Nikos Giokaris for their careful reading of our draft paper on this work for the publication and providing us many precious comments. I also thank Chang Paoti and other members of the QCD analysis group for their helpful advice.

Howard Budd, Yasuo Fukui, Tomohiro Kaneko, Masanori Mishina and Fan Qun are appreciated for their help to my work on the detector at CDF.

Discussion on physics and other various things with Shinhong Kim, Satoru Ogawa, Ryutaro Oishi, Yoshihiro Seiya, Mikio Takano and Fumihiko Ukegawa were very beneficial for me. Conversations with Junsuke Iwai, Kaori Maeshima and Nobuaki Oshima made my stay at Fermilab unforgettable. I was very fortunate to have a good time with Tsuyoshi Takada and Eiichiro Hayashi in Aurora.

I should thank Shin Aota, Hosai Nakada, Junichi Suzuki, Hirotoshi Toyoda and other my colleagues staying at Fermilab for their help. I also wish to thank other members of the Tsukuba high energy physics group for their constant help. They include Kazuhiko Hara, Shigeyuki Miyashita, Itsuo Nakano, Kiyoshi Yasuoka and other colleagues.

I thank the Fermilab staffs and the technical staffs of the participating institutions for their vital contributions. I thank many CDF collaborators who have developed the 
detector and other analysis environment.

I want to express my great appreciation to Carol Picciolo, Kyoko Kunori, Mutsumi Uenishi and Kazuko Kumashiro for their help through their secretary works.

Finally and most importantly I thank my parents and my brother for their constant support and encouragement.

This work was supported by the U.S. Department of Energy and National Science Foundation; the Italian Istituto Nazionale di Fisica Nucleare; the Ministry of Education, Science and Culture of Japan; the Natural Sciences and Engineering Research Council of Canada; the National Science Council of the Republic of China; and the A. P. Sloan Foundation. 


\section{Contents}

1 Introduction $\quad \mathbf{2 2}$

1.1 Quantum Chromodynamics .................. 23

1.2 Theoretical Models for Multijet Production . . . . . . . . . . 25

1.3 Analysis Overview .................... 25

2 Apparatus $\quad 31$

2.1 The Fermilab Tevatron $\bar{p} p$ Collider . . . . . . . . . . . . . . . 31

2.2 The CDF Detector . . . . . . . . . . . . . . . . 32

2.2.1 Beam-Beam Counter . . . . . . . . . . . . . . 33

2.2 .2 Tracking Detector . . . . . . . . . . . . . . 33

2.2 .3 Muon Detector . . . . . . . . . . . . . . . . . 34

2.2 .4 Calorimetry . . . . . . . . . . . . . . . 34

3 Data Set 41

3.1 Data Acquisition . . . . . . . . . . . . . . . 41

3.1 .1 CDF Data Flow . . . . . . . . . . . . . . . . . 41

3.1 .2 Definition of Jet . . . . . . . . . . . . . . 41

$3.1 .3 \sum E_{T}$ trigger . . . . . . . . . . . . . . 43

$3.2 \sum E_{T}$ Data Sample . . . . . . . . . . . . . . . 44

3.2 .1 Jet Energy Corrections . . . . . . . . . . . . . . 45

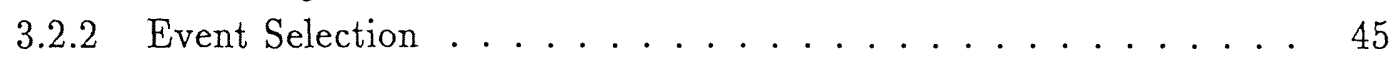

3.2.3 Further Selection of Multijet Events . . . . . . . . . . . . 47

4 Theoretical Predictions $\quad 55$

4.1 HERWIG parton shower Monte Carlo calculation . . . . . . . . 55

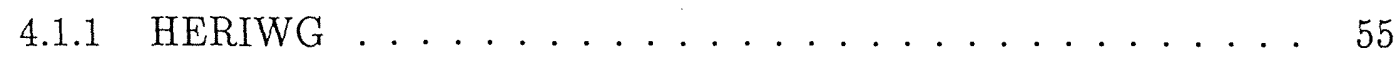

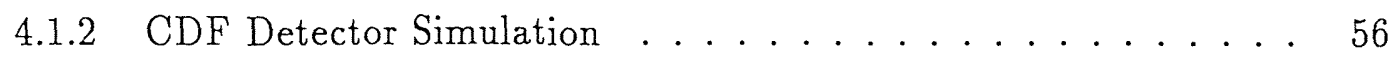

4.2 NJETS QCD matrix element calculation . . . . . . . . . . 57

4.3 Phase-Space Model . . . . . . . . . . . . . . . 57 
5 Analysis $\quad 59$

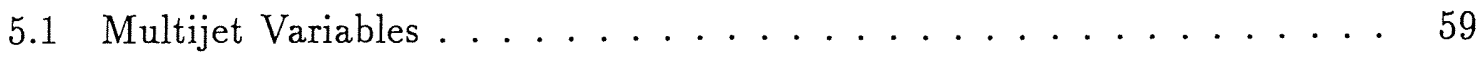

5.1 .1 Average Beam Direction . . . . . . . . . . . . 60

5.1 .2 Three-jet Variables . . . . . . . . . . . . . . . . . 60

5.1 .3 Four-jet Variables . . . . . . . . . . . . . 62

5.1 .4 Five-jet Variables . . . . . . . . . . . . . . . 64

5.1.5 Generalization to Events with Six or More Jets . . . . . . . . . 67

5.2 Event Topology Cuts . . . . . . . . . . . . . . . . . 69

5.2.1 Leading Body Scattering Angle and Multijet Mass . . . . . . . . 69

5.2 .2 Dalitz Variable . . . . . . . . . . . . . . . 70

6 Results and Discussion $\quad 78$

6.1 Multijet-mass Distributions . . . . . . . . . . . 78

6.2 Three-Body Distributions . . . . . . . . . . . . . . 79

6.2.1 Three-Body Dalitz Distributions . . . . . . . . . . . . 79

6.2.2 Three-Body Angular Distributions . . . . . . . . . . . 81

6.2.3 Single-Body Mass Distributions for Three-Body Systems . . . . 82

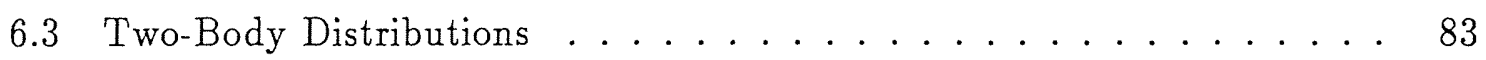

6.3.1 Two-Body Energy Sharing Distributions . . . . . . . . 83

6.3.2 Two-Body Angular Distributions ... . . . . . . . 83

6.3.3 Single-Body Mass Distributions for Two-Body Systems . . . . . 84

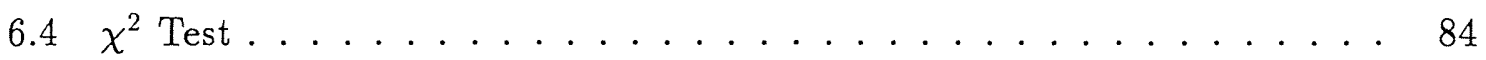

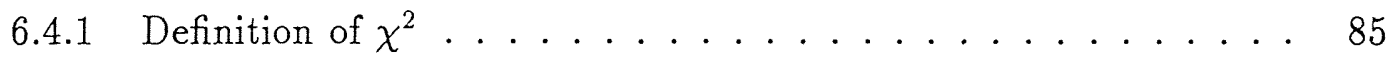

6.4 .2 Results of the $\chi^{2}$ Test . . . . . . . . . . . 85

6.4 .3 Event Rates ................... 86

7 Systematic Studies $\quad 108$

7.1 Theoretical Uncertainties . . . . . . . . . . . . . . . 108

7.2 Influence from Multiple Interactions . . . . . . . . . . . . . . 110

7.3 Jet Energy Scale . . . . . . . . . . . . . . . . . . . . 112

8 Conclusions $\quad 145$

$\begin{array}{lr}\text { A Event Pictures } & 149\end{array}$

B Validity of our $\chi^{2}$ test 155

$\begin{array}{ll}\text { C Analytic Form of } X \text { Variable } & 157\end{array}$ 


\section{List of Figures}

1.1 Examples of the Feynman diagrams for $2 \rightarrow 3$ subprocesses. . . . . . 28

1.2 Examples of the Feynman diagrams for $2 \rightarrow 4$ subprocesses. . . . . . 29

1.3 Examples of the Feynman diagrams for $2 \rightarrow 5$ subprocesses. . . . . . 30

2.1 The perspective view of the Tevatron Proton-Antiproton Collider at Fermi National Accelerator Laboratory. . . . . . . . . . . . . . . . 38

2.2 Perspective view of the Collider Detector at Fermilab. . . . . . . . . . 39

2.3 The $\eta-\phi$ segmentation of the CDF calorimeter. Also shown is the size of a cone cluster with a 0.7 radius. This is the cone clustering radius used in our analysis. . . . . . . . . . . . . . 40

3.1 The observed distribution of $\Delta R$ between all jet-jet pairs in inclusive five-jet events. . . . . . . . . . . . . . . . . 49

3.2 The cross-section distributions of the Level 2 and Level $3 \sum E_{T}$ triggers as a fuction of run number. . . . . . . . . . . . 50

3.3 The observed missing $E_{T}$ distributions: (a) the observed missing $E_{T}$ significance distribution (points) and (b) the observed missing $E_{T}$ distribution (points) compared with the HERWIG predictions (histogram). 51

3.4 The observed $\sum E_{T}$ distributions: (a) the observed distribution (points) and (b) the observed distribution (points) compared with the HERWIG prediction (histograms) after applying the requirement of $\sum E_{T}>420$

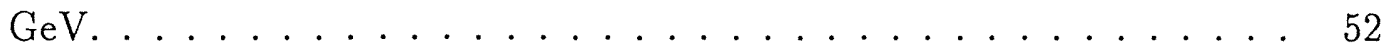

3.5 The reconstructed vertex ( $z$ position) distribution of the observed events. 53

3.6 The scatter plots of the corrected and uncorrrected $\sum E_{T} \ldots \ldots . . .54$

5.1 Schematic definition of angles used to describe the three-jet system in the three-jet rest-frame. . . . . . . . . . . . . 72 
5.2 Schematic definition of angles used to describe the four-jet system in the four-jet rest-frame. . . . . . . . . . . . . .

5.3 Schematic definition of angles used to describe the five-jet system in the five-jet rest-frame. . . . . . . . . . . . . . . .

5.4 Observed distributions of (a) $m_{3 J}$ vs. $\left|\cos \theta_{3}\right|$, (b) $m_{4 J}$ vs. $\left|\cos \theta_{3^{\prime}}\right|$, and (c) $m_{5 J}$ vs. $\left|\cos \theta_{3^{\prime \prime}}\right|$ after applying the $\sum E_{T}$ requirement. . . . . . .

5.5 Observed distributions of inclusive multijet mass for topologies with (a) three jets, (b) four jets, and (c) five jets. . . . . . . . . . .

5.6 Observed distributions of Dalitz variables: (a) $X_{3^{\prime}}$, (b) $X_{3^{\prime \prime}}$, and (c) $X_{3^{\prime \prime}}$ after applying the requirements on $m_{N J}$ and $|\cos \theta|$ variables. . . . 77

6.1 Inclusive multijet mass distributions for topologies with (a) three jets, (b) four jets, and (c) five jets. Observed distributions (points) are compared with HERWIG predictions (triangles) and NJETS predictions (squares). . . . . . . . . . . . . . . .

6.2 Three-jet Dalitz distributions after imposing the requirements $m_{3 J}>$ $600 \mathrm{GeV} / c^{2}, X_{3}<0.9$, and $\left|\cos \theta_{3}\right|<0.6$, shown for (a) data, (b) NJETS, (c) HERWIG, and (d) the phase-space model. . . . . . . . . .

6.3 Inclusive three-jet Dalitz distributions for events that satisfy the requirements $m_{3 J}>600 \mathrm{GeV} / c^{2}, X_{3}<0.9$, and $\left|\cos \theta_{3}\right|<0.6$. Data (points) are compared with HERWIG predictions (triangles), NJETS predictions (squares), and phase-space model predictions (curves) for (a) $X_{3}$, and (b) $X_{4} \ldots \ldots \ldots \ldots \ldots \ldots \ldots \ldots \ldots \ldots \ldots \ldots \ldots \ldots \ldots \ldots \ldots$

6.4 Inclusive four-jet Dalitz distributions for events that that satisfy the requirements $m_{4 J}>650 \mathrm{GeV} / c^{2}, X_{3^{\prime}}<0.9$, and $\left|\cos \theta_{3^{\prime}}\right|<0.8$, shown for (a) data, (b) NJETS, (c) HERWIG, and (d) phase-space model predictions. 92

6.5 Dalitz distributions for inclusive four-jet topologies that satisfy the requirements $m_{4 J}>650 \mathrm{GeV} / c^{2}, X_{3^{\prime}}<0.9$, and $\left|\cos \theta_{3^{\prime}}\right|<0.8$. Data (points) are compared with HERWIG predictions (triangles), NJETS predictions (squares), and phase-space model predictions (curves) for (a) $X_{3^{\prime}}$, and (b) $X_{4^{\prime}} \ldots \ldots \ldots \ldots \ldots \ldots$

6.6 Inclusive five-jet Dalitz distributions for events that satisfy the requirement $m_{5 J}>750 \mathrm{GeV} / c^{2}$, shown for (a) data, (b) NJETS, (c) HERWIG, and $(\mathrm{d})$ phase-space model predictions. 
6.7 Dalitz distributions for inclusive five-jet topologies that satisfy the requirement $m_{5 J}>750 \mathrm{GeV} / c^{2}$. Data (points) are compared with HERWIG predictions (triangles), NJETS predictions (squares), and phasespace model predictions (curves) for (a) $X_{3^{\prime \prime}}$, and (b) $X_{4^{\prime \prime}}$. . . . . .

6.8 Inclusive three-jet angular distributions for events that satisfy the requirements $m_{3 J}>600 \mathrm{GeV} / c^{2}, X_{3}<0.9$, and $\left|\cos \theta_{3}\right|<0.6$. Event populations in the $\left(\cos \theta_{3}, \psi_{3}\right)$-plane are shown for (a) data, (b) NJETS, (c) HERWIG, and (d) phase-space model predictions. . . . . . . . .

6.9 Inclusive three-jet angular distributions for events that satisfy the requirements $m_{3 J}>600 \mathrm{GeV} / c^{2}, X_{3}<0.9$, and $\left|\cos \theta_{3}\right|<0.6$. Data (points) are compared with HERWIG predictions (triangles), NJETS predictions (squares), and phase-space model predictions (curves) for (a) $\cos \theta_{3}$ and (b) $\psi_{3}$. The broken curve in the $\cos \theta_{3}$ figure is the LO QCD prediction for $q \bar{q} \rightarrow q \bar{q}$ scattering. . . . . . . . . . .

6.10 Inclusive four-jet angular distributions for events that satisfy the requirements $m_{4 J}>650 \mathrm{GeV} / c^{2}, X_{3^{\prime}}<0.9$, and $\left|\cos \theta_{3^{\prime}}\right|<0.8$. Event populations in the $\left(\cos \theta_{3^{\prime}}, \psi_{3^{\prime}}\right)$-plane are shown for (a) data, (b) NJETS, (c) HERWIG, and (d) phase-space model predictions. . . . . . . . . .

6.11 Inclusive four-jet angular distributions for events that satisfy the requirements $m_{4 J}>650 \mathrm{GeV} / c^{2}, X_{3^{\prime}}<0.9$, and $\left|\cos \theta_{3^{\prime}}\right|<0.8$. Data (points) are compared with HERWIG predictions (triangles), NJETS predictions (squares), and phase-space model predictions (curves) for (a) $\cos \theta_{3^{\prime}}$ and (b) $\psi_{3^{\prime}}$. The broken curve in the $\cos \theta_{3^{\prime}}$ figure is the LO QCD prediction for $q \bar{q} \rightarrow q \bar{q}$ scattering. . . . . . . . . . . . . .

6.12 Inclusive five-jet angular distributions for events that satisfy the requirement $m_{5 J}>750 \mathrm{GeV} / c^{2}$. Event populations in the $\left(\cos \theta_{3^{\prime \prime}}, \psi_{3^{\prime \prime}}\right)$-plane are shown for (a) data, (b) NJETS, (c) HERWIG, and (d) phase-space model predictions. . . . . . . . . . . . . . . 100

6.13 Inclusive five-jet angular distributions for events that satisfy the requirement $m_{5 J}>750 \mathrm{GeV} / c^{2}$. Data (points) are compared with HERWIG predictions (triangles), NJETS predictions (squares), and phase-space model predictions (curves) for (a) $\cos \theta_{3^{\prime \prime}}$ and (b) $\psi_{3^{\prime \prime}}$. The broken curve in the $\cos \theta_{3^{\prime \prime}}$ figure is the LO QCD prediction for $q \bar{q} \rightarrow q \bar{q}$ scattering. . 101 
6.14 Single-jet mass fraction distributions for inclusive three-jet events. Data (points) compared with HERWIG predictions (triangles), shown for (a) the highest energy jet in the three-jet rest-frame, (b) the second-tohighest energy jet, and (c) the third-to-highest energy jet. . . . . . . 102

6.15 Single-body mass fraction distributions for inclusive four-jet events. Data (points) compared with HERWIG predictions (triangles), and NJETS predictions (histograms), shown for (a) the highest energy body in the three-body rest-frame, (b) the second-to-highest energy body, and (c) the third-to-highest energy body. . . . . . . . . . . . . 103

6.16 Single-body mass fraction distributions for inclusive five-jet events. Data (points) compared with HERWIG predictions (triangles), and NJETS predictions (histograms), shown for (a) the highest energy body in the three-body rest-frame, (b) the second-to-highest energy body, and (c) the third-to-highest energy body. . . . . . . . . . . . . . . 104

6.17 The two-body energy sharing distributions for inclusive four-jet and five-jet events. Data (points) are compared with HERWIG predictions (triangles), NJETS predictions (squares), and phase-space predictions (curves) for (a) $X_{A}$, (b) $X_{A}$ after dividing by the phase-space model predictions, (c) $X_{A^{\prime}}$, (d) $X_{A^{\prime}}$ after dividing by the phase-space model predictions, (e) $X_{C}$, and (f) $X_{C}$ after dividing by the phase-space model predictions. . . . . . . . . . . . . . 105

6.18 Two-body angular distributions for inclusive four-jet and five-jet events. Data (points) are compared with HERWIG predictions (triangles), NJETS predictions (squares), and phase-space predictions (curves) for (a) $\psi_{A B}^{\prime}$, (b) $\psi_{A B}^{\prime}$ after dividing by the phase-space model predictions, (c) $\psi_{A^{\prime} B^{\prime}}^{\prime \prime}$, (d) $\psi_{A^{\prime} B^{\prime}}^{\prime \prime}$ after dividing by the phase-space model predictions, (e) $\psi_{C D}^{\prime \prime}$, and (f) $\psi_{C D}^{\prime \prime}$ after dividing by the phase-space model predictions. . . . . 106

6.19 Single-body mass fraction distributions for two-body systems in inclusive four-jet and five-jet events. Data (points) are compared with HERWIG predictions (triangles), and NJETS predictions (histograms) for (a) $f_{A}$, (b) $f_{B}$, (c) $f_{A^{\prime}}$, (d) $f_{B^{\prime}}$, (e) $f_{C}$, and (f) $f_{D} \ldots \ldots \ldots$ 
7.1 The NJETS predictions of the leading 3-jet mass distribution for various choices of structure functions, $Q^{2}$-scales, and $\Delta R_{\min }$. Eight curves in the figure correspond to the eight choices listed in Table 7.1. The histogram is the measured $m_{3 J}$ distribution. . . . . . . . . . . .

7.2 The NJETS predictions of the three-body distributions of 3 -jet events for various choices of structure functions, $Q^{2}$-scales, and $\Delta R_{\min }$. Eight curves in each figure correspond to the eight choices listed in Table 7.1. Histograms are the measured distribution. (a) $X_{3}$, (b) $X_{4}$, (c) $\cos \theta_{3}$, and $(\mathrm{d}) \psi_{3} \ldots \ldots \ldots \ldots \ldots \ldots \ldots$

7.3 The NJETS predictions of the $m_{4 J}$ distribution of 4-jet events for various choices of structure functions, $Q^{2}$-scales, and $\Delta R_{\min }$. Eight curves in the figure correspond to the eight choices listed in Table 7.1. The histogram is the measured $m_{4 J}$ distribution. . . . . . . . . . . . . . . 116

7.4 The NJETS predictions of the three-body distributions of 4-jet events for various choices of structure functions, $Q^{2}$-scales, and $\Delta R_{\min }$. Eight curves in each figure correspond to the eight choices listed in Table 7.1. Histograms are the measured distribution. (a) $X_{3^{\prime}}$, (b) $X_{4^{\prime}}$, (c) $\cos \theta_{3^{\prime}}$,

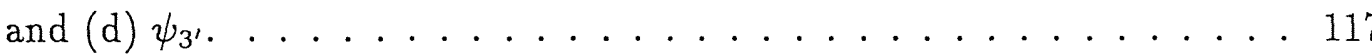

7.5 The NJETS predictions of the $f_{J^{\prime}}\left(J^{\prime}=3^{\prime}, 4^{\prime}, 5^{\prime}\right)$ distributions of 4 -jet events for various choices of structure functions, $Q^{2}$-scales, and $\Delta R_{\min }$. Eight curves in each figure correspond to the eight choices listed in Table 7.1. Histograms are the measured distribution. (a) $f_{3^{\prime}}$, (b) $f_{4^{\prime}}$, and (c)

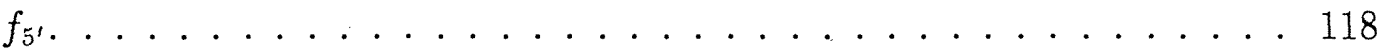

7.6 The NJETS predictions of the two $(A+B)$-body distributions of 4-jet events for various choices of structure functions, $Q^{2}$-scales, and $\Delta R_{\min }$. Eight curves in each figure correspond to the eight choices listed in Table 7.1. Histograms are the measured distribution. (a) $X_{A^{\prime}}$, (b) $\psi_{A B}^{\prime}$. . . 119

7.7 The instantaneous luminosity distribution. The multijet data sample was separated into three subsamples containing events detected at low luminosities $\left(\mathcal{L}<4 \times 10^{30} \mathrm{~cm}^{-2} \mathrm{~s}^{-1}\right)$, intermediate luminosities $(4<\mathcal{L}<$ $\left.8 \times 10^{30} \mathrm{~cm}^{-2} \mathrm{~s}^{-1}\right)$, and high luminosities $\left(\mathcal{L}>8 \times 10^{30} \mathrm{~cm}^{-2} \mathrm{~s}^{-1}\right) . \ldots 120$ 
7.8 The observed $m_{3 J}$ distributions of 3 -jet events in three different ranges of instantaneous luminosities. In the figure the histogram corresponds to the lowest luminosity range $\left(\mathcal{L}<4 \times 10^{30} \mathrm{~cm}^{-2} \mathrm{~s}^{-1}\right)$, points correspond to the intermediate luminosity range $\left(4<\mathcal{L}<8 \times 10^{30} \mathrm{~cm}^{-2} \mathrm{~s}^{-1}\right)$, and squares correspond to the highest luminosity range $\left(\mathcal{L}>8 \times 10^{30}\right.$ $\mathrm{cm}^{-2} \mathrm{~s}^{-1}$ ), respectively. . . . . . . . . . . . . . 121

7.9 The observed three-body distributions of 3 -jet events in three different ranges of instantaneous luminosities. In the figure the histograms correspond to the lowest luminosity range $\left(\mathcal{L}<4 \times 10^{30} \mathrm{~cm}^{-2} \mathrm{~s}^{-1}\right)$, points correspond to the intermediate luminosity range $\left(4<\mathcal{L}<8 \times 10^{30}\right.$ $\left.\mathrm{cm}^{-2} \mathrm{~s}^{-1}\right)$, and squares correspond to the highest luminosity range $(\mathcal{L}>$ $8 \times 10^{30} \mathrm{~cm}^{-2} \mathrm{~s}^{-1}$ ), respectively. (a) $X_{3}$, (b) $X_{4}$, (c) $\cos \theta_{3}$, and (d) $\psi_{3}$. .

7.10 The observed $f_{J}(J=3,4,5)$ distributions of 3 -jet events in three different ranges of instantaneous luminosities. In the figure the histograms correspond to the lowest luminosity range $\left(\mathcal{L}<4 \times 10^{30} \mathrm{~cm}^{-2} \mathrm{~s}^{-1}\right)$, points correspond to the intermediate luminosity range $\left(4<\mathcal{L}<8 \times 10^{30}\right.$ $\left.\mathrm{cm}^{-2} \mathrm{~s}^{-1}\right)$, and squares correspond to the highest luminosity range $(\mathcal{L}>$ $8 \times 10^{30} \mathrm{~cm}^{-2} \mathrm{~s}^{-1}$ ), respectively. (a) $f_{3}$, (b) $f_{4}$, and (c) $f_{5} \ldots \ldots$

7.11 The observed $m_{4 J}$ distributions in three different ranges of instantaneous luminosities. In the figure the histogram corresponds to the lowest luminosity range $\left(\mathcal{L}<4 \times 10^{30} \mathrm{~cm}^{-2} \mathrm{~s}^{-1}\right)$, points correspond to the intermediate luminosity range $\left(4<\mathcal{L}<8 \times 10^{30} \mathrm{~cm}^{-2} \mathrm{~s}^{-1}\right)$, and squares correspond to the highest luminosity range $\left(\mathcal{L}>8 \times 10^{30} \mathrm{~cm}^{-2} \mathrm{~s}^{-1}\right)$, respectively. . . . . . . . . . . . . . . . . . . . . . . 124

7.12 The observed three-body distributions of 4-jet events in three different ranges of instantaneous luminosities. In the figure the histograms correspond to the lowest luminosity range $\left(\mathcal{L}<4 \times 10^{30} \mathrm{~cm}^{-2} \mathrm{~s}^{-1}\right)$, points correspond to the intermediate luminosity range $\left(4<\mathcal{L}<8 \times 10^{30}\right.$ $\left.\mathrm{cm}^{-2} \mathrm{~s}^{-1}\right)$, and squares correspond to the highest luminosity range $(\mathcal{L}\rangle$ $8 \times 10^{30} \mathrm{~cm}^{-2} \mathrm{~s}^{-1}$ ), respectively. (a) $X_{3^{\prime}}$, (b) $X_{4^{\prime}}$, (c) $\cos \theta_{3^{\prime}}$, and (d) $\psi_{3^{\prime}}$. 
7.13 The observed $f_{J^{\prime}}\left(J^{\prime}=3^{\prime}, 4^{\prime}, 5^{\prime}\right)$ distributions of 4 -jet events in three different ranges of instantaneous luminosities. In the figure the histograms correspond to the lowest luminosity range $\left(\mathcal{L}<4 \times 10^{30} \mathrm{~cm}^{-2} \mathrm{~s}^{-1}\right)$, points correspond to the intermediate luminosity range $\left(4<\mathcal{L}<8 \times 10^{30}\right.$ $\left.\mathrm{cm}^{-2} \mathrm{~s}^{-1}\right)$, and squares correspond to the highest luminosity range $(\mathcal{L}>$ $8 \times 10^{30} \mathrm{~cm}^{-2} \mathrm{~s}^{-1}$ ), respectively. (a) $f_{3^{\prime}}$, (b) $f_{4^{\prime}}$, and (c) $f_{5^{\prime}} \ldots \ldots$

7.14 The observed two $(A+B)$-body distributions of 4-jet events in three different ranges of instantaneous luminosities. In the figure the histograms correspond to the lowest luminosity range $\left(\mathcal{L}<4 \times 10^{30} \mathrm{~cm}^{-2} \mathrm{~s}^{-1}\right)$, points correspond to the intermediate luminosity range $\left(4<\mathcal{L}<8 \times 10^{30}\right.$ $\left.\mathrm{cm}^{-2} \mathrm{~s}^{-1}\right)$, and squares correspond to the highest luminosity range $(\mathcal{L}>$ $8 \times 10^{30} \mathrm{~cm}^{-2} \mathrm{~s}^{-1}$ ), respectively. (a) $X_{A}$, (b) $\psi_{A B}^{\prime}$, (c) $f_{A}$, and (d) $f_{B}$. .

7.15 The observed $m_{5 J}$ distributions in three different ranges of instantaneous luminosities. In the figure the histogram corresponds to the lowest luminosity range $\left(\mathcal{L}<4 \times 10^{30} \mathrm{~cm}^{-2} \mathrm{~s}^{-1}\right)$, points correspond to the intermediate luminosity range $\left(4<\mathcal{L}<8 \times 10^{30} \mathrm{~cm}^{-2} \mathrm{~s}^{-1}\right)$, and squares correspond to the highest luminosity range $\left(\mathcal{L}>8 \times 10^{30} \mathrm{~cm}^{-2} \mathrm{~s}^{-1}\right)$, respectively. . . . . . . . . . . . . . . . 128

7.16 The observed three-body distributions of 5 -jet events in three different ranges of instantaneous luminosities. In the figure the histograms correspond to the lowest luminosity range $\left(\mathcal{L}<4 \times 10^{30} \mathrm{~cm}^{-2} \mathrm{~s}^{-1}\right)$, points correspond to the intermediate luminosity range $\left(4<\mathcal{L}<8 \times 10^{30}\right.$ $\left.\mathrm{cm}^{-2} \mathrm{~s}^{-1}\right)$, and squares correspond to the highest luminosity range $(\mathcal{L}>$ $8 \times 10^{30} \mathrm{~cm}^{-2} \mathrm{~s}^{-1}$ ), respectively. (a) $X_{3^{\prime \prime}}$, (b) $X_{4^{\prime \prime}}$, (c) $\cos \theta_{3^{\prime \prime}}$, and (d) $\psi_{3^{\prime \prime}} .129$

7.17 The observed $f_{J^{\prime \prime}}\left(J^{\prime \prime}=3^{\prime \prime}, 4^{\prime \prime}, 5^{\prime \prime}\right)$ distributions of 5 -jet events in three different ranges of instantaneous luminosities. In the figure the histograms correspond to the lowest luminosity range $\left(\mathcal{L}<4 \times 10^{30} \mathrm{~cm}^{-2} \mathrm{~s}^{-1}\right)$, points correspond to the intermediate luminosity range $\left(4<\mathcal{L}<8 \times 10^{30}\right.$ $\left.\mathrm{cm}^{-2} \mathrm{~s}^{-1}\right)$, and squares correspond to the highest luminosity range $(\mathcal{L}>$ $8 \times 10^{30} \mathrm{~cm}^{-2} \mathrm{~s}^{-1}$ ), respectively. (a) $f_{3^{\prime \prime}}$, (b) $f_{4^{\prime \prime}}$, and (c) $f_{5^{\prime \prime}} \ldots \ldots 130$ 
7.18 The observed two $\left(A^{\prime}+B^{\prime}\right)$-body distributions of 5-jet events in three different ranges of instantaneous luminosities. In the figure the histograms correspond to the lowest luminosity range $\left(\mathcal{L}<4 \times 10^{30} \mathrm{~cm}^{-2} \mathrm{~s}^{-1}\right)$, points correspond to the intermediate luminosity range $\left(4<\mathcal{L}<8 \times 10^{30}\right.$ $\left.\mathrm{cm}^{-2} \mathrm{~s}^{-1}\right)$, and squares correspond to the highest luminosity range $(\mathcal{L}>$ $8 \times 10^{30} \mathrm{~cm}^{-2} \mathrm{~s}^{-1}$ ), respectively. (a) $X_{A^{\prime}}$, (b) $\psi_{A^{\prime} B^{\prime}}^{\prime \prime}$, (c) $f_{A^{\prime}}$, and (d) $f_{B^{\prime}} .131$

7.19 The observed two $(C+D)$-body distributions of 5 -jet events in three different ranges of instantaneous luminosities. In the figure the histograms correspond to the lowest luminosity range $\left(\mathcal{L}<4 \times 10^{30} \mathrm{~cm}^{-2} \mathrm{~s}^{-1}\right)$, points correspond to the intermediate luminosity range $\left(4<\mathcal{L}<8 \times 10^{30}\right.$ $\left.\mathrm{cm}^{-2} \mathrm{~s}^{-1}\right)$, and squares correspond to the highest luminosity range $(\mathcal{L}>$ $8 \times 10^{30} \mathrm{~cm}^{-2} \mathrm{~s}^{-1}$ ), respectively. (a) $X_{C},(\mathrm{~b}) \psi_{C D}^{\prime \prime}$, (c) $f_{C}$, and (d) $f_{D} \cdot 132$

7.20 The observed $m_{3 J}$ distributions for three data samples in which the jet energies were scaled by $-7 \%, 0 \%$, and $+7 \%$. In the figure the histograms corresponds to events with the nominal energy scale, points and squares correspond to events with energies shifted by $-7 \%$ and $-7 \%$, respectively. 133

7.21 The observed three-body distributions of 3 -jet events for three data samples in which the jet energies were scaled by $-7 \%, 0 \%$, and $+7 \%$. In the figure histograms correspond to events with the nominal energy scale, points and squares correspond to events with energies shifted by $-7 \%$ and $-7 \%$, respectively. (a) $X_{3}$, (b) $X_{4}$, (c) $\cos \theta_{3}$, and (d) $\psi_{3} \ldots \ldots 134$

7.22 The observed $f_{J}(J=3,4,5)$ distributions of 3 -jet events for three data samples in which the jet energies were scaled by $-7 \%, 0 \%$, and $+7 \%$. In the figure histograms correspond to events with the nominal energy scale, points and squares correspond to events with energies shifted by $-7 \%$ and $-7 \%$, respectively. (a) $f_{3}$, (b) $f_{4}$, and (c) $f_{5} \ldots \ldots 135$

7.23 The observed $m_{4 J}$ distributions for three data samples in which the jet energies were scaled by $-7 \%, 0 \%$, and $+7 \%$. In the figure the histogram corresponds to events with the nominal energy scale, points and squares correspond to events with energies shifted by $-7 \%$ and $-7 \%$, respectively. 136 
7.24 The observed three-body distributions of 4-jet events for three data samples in which the jet energies were scaled by $-7 \%, 0 \%$, and $+7 \%$. In the figure the histograms correspond to events with the nominal energy scale, points and squares correspond to events with energies shifted by $-7 \%$ and $-7 \%$, respectively. (a) $X_{3^{\prime}}$, (b) $X_{4^{\prime}}$, (c) $\cos \theta_{3^{\prime}}$, and (d) $\psi_{3^{\prime}}$.

7.25 The observed $f_{J^{\prime}}\left(J^{\prime}=3^{\prime}, 4^{\prime}, 5^{\prime}\right)$ distributions of 4 -jet events for three data samples in which the jet energies were scaled by $-7 \%, 0 \%$, and $+7 \%$. In the figure the histograms correspond to events with the nominal energy scale, points and squares correspond to events with energies shifted by $-7 \%$ and $-7 \%$, respectively. (a) $f_{3^{\prime}}$, (b) $f_{4^{\prime}}$, and (c) $f_{5^{\prime}} \ldots$

7.26 The observed two $(A+B)$-body distributions of 4 -jet events for three data samples in which the jet energies were scaled by $-7 \%, 0 \%$, and $+7 \%$. In the figure the histograms correspond to events with the nominal energy scale, points and squares correspond to events with energies shifted by $-7 \%$ and $-7 \%$, respectively. (a) $X_{A}$, (b) $\psi_{A B}^{\prime}$, (c) $f_{A}$, and (d) $f_{B} \ldots \ldots \ldots \ldots \ldots \ldots \ldots$

7.27 The observed $m_{5 J}$ distributions of 5 -jet events for three data samples in which the jet energies were scaled by $-7 \%, 0 \%$, and $+7 \%$. In the figure the histogram corresponds to events with the nominal energy scale, points and squares correspond to events with energies shifted by $-7 \%$

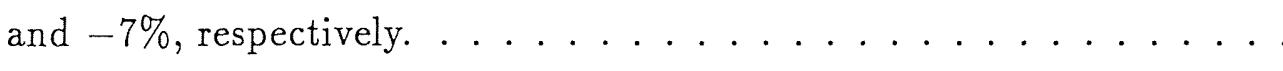

7.28 The observed three-body distributions of 5-jet events for three data samples in which the jet energies were scaled by $-7 \%, 0 \%$, and $+7 \%$. In the figure the histograms correspond to events with the nominal energy scale, points and squares correspond to events with energies shifted by $-7 \%$ and $-7 \%$, respectively. (a) $X_{3^{\prime \prime}}$, (b) $X_{4^{\prime \prime}}$, (c) $\cos \theta_{3^{\prime \prime}}$, and (d) $\psi_{3^{\prime \prime}}$.

7.29 The observed $f_{J^{\prime \prime}}\left(J^{\prime \prime}=3^{\prime \prime}, 4^{\prime \prime}, 5^{\prime \prime}\right)$ distributions of 5 -jet events for three data samples in which the jet energies were scaled by $-7 \%, 0 \%$, and $+7 \%$. In the figure the histograms correspond to events with the nominal energy scale, points and squares correspond to events with energies shifted by $-7 \%$ and $-7 \%$, respectively. (a) $f_{3^{\prime \prime}}$, (b) $f_{4^{\prime \prime}}$, and (c) $f_{5^{\prime \prime}}$. . 142 
7.30 The observed two $\left(A^{\prime}+B^{\prime}\right)$-body distributions of 5 -jet events for three data samples in which the jet energies were scaled by $-7 \%, 0 \%$, and $+7 \%$. In the figure the histograms correspond to events with the nominal energy scale, points and squares correspond to events with energies shifted by $-7 \%$ and $-7 \%$, respectively. (a) $X_{A^{\prime}}$, (b) $\psi_{A^{\prime} B^{\prime}}^{\prime \prime}$, (c) $f_{A^{\prime}}$, and

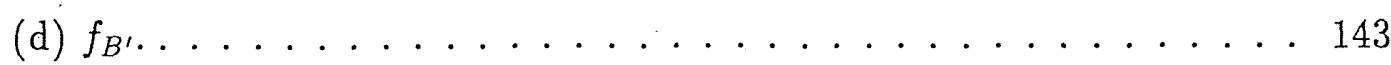

7.31 The observed two $(C+D)$-body distributions of 5 -jet events for three data samples in which the jet energies were scaled by $-7 \%, 0 \%$, and $+7 \%$. In the figure the histograms correspond to events with the nominal energy scale, points and squares correspond to events with energies shifted by $-7 \%$ and $-7 \%$, respectively. (a) $X_{C}$, (b) $\psi_{C D}^{\prime \prime}$, (c) $f_{C}$, and

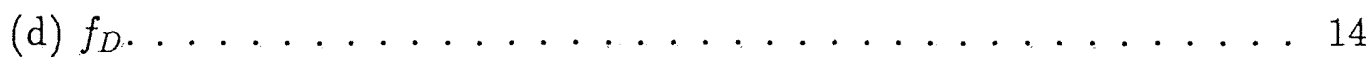

A.1 Event Pictures for events with 3 Jets and (a) $X_{3}=0.89$ and $X_{4}=0.89$, (b) $X_{3}=0.89$ and $X_{4}=0.57$, (c) $X_{3}=0.68$ and $X_{4}=0.66$, and (d) $X_{3}$ $=0.85$ and $X_{4}=0.70 \ldots \ldots \ldots \ldots$

A.2 Event Pictures for events with 4 Jets and (a) $X_{3^{\prime}}=0.90$ and $X_{4^{\prime}}=0.88$, (b) $X_{3^{\prime}}=0.87$ and $X_{4^{\prime}}=0.57$, (c) $X_{3^{\prime}}=0.68$ and $X_{4^{\prime}}=0.67$, and (d) $X_{3^{\prime}}=0.84$ and $X_{4^{\prime}}=0.71 \ldots \ldots \ldots \ldots \ldots$

A.3 Event Pictures for events with 5 Jets and (a) $X_{3^{\prime \prime}}=0.99$ and $X_{4^{\prime \prime}}=$ 0.90 , (b) $X_{3^{\prime \prime}}=0.99$ and $X_{4^{\prime \prime}}=0.55$, (c) $X_{3^{\prime \prime}}=0.71$ and $X_{4^{\prime \prime}}=0.67$, and (d) $X_{3^{\prime \prime}}=0.87$ and $X_{4^{\prime \prime}}=0.70$............... 154

B.1 The $\chi^{2}$ distributions. $\chi_{2}^{2}$ (points) compared with $\chi_{1}^{2}$ (histogram) and $\chi_{\exp }^{2}$ (curve). (a), (b), (c) and (d) are for the number of bins $=5,10$,

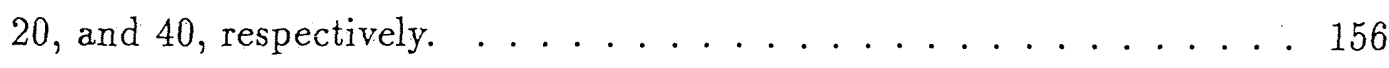




\section{List of Tables}

1.1 Lowest order parton-parton cross-sections. . . . . . . . . . . . 26

2.1 Summary of CDF calorimeter properties. The symbol $\oplus$ signifies that the constant term is added in quadrature in the resolution. Energy resolutions for the electromagnetic calorimeters are for incident electrons and photons, and for hadronic calorimeters are for incident isolated pions. Energy given in GeV. Thicknesses are given in radiation lengths $\left(X_{0}\right)$ and interaction lengths $(\lambda)$ for the electromagnetic and hadronic calorimeters, respectively.

3.1 Numbers of events remaining after the multijet event selection (1st column) and after applying the cuts on multijet variables (2nd column).

5.1 Summary of the (4N-4) multijet variables for $\mathrm{N}=3,4,5$, and $6 \ldots \ldots 8$

5.2 Event topology cuts on three-, four-, and five-jet variables. These requirements reduce, (but do not completely eliminate), the regions of low experimental acceptance. . . . . . . . . . . . 71

6.1 Fit results from the exponential fits to the multijet mass distributions.

6.2 Statistical comparison of agreement between observed and predicted distributions of the three-jet variables. The $\chi^{2}$ s per degree of freedom are listed. In calculating the over all $\chi^{2} \mathrm{~s}$, the single body mass fraction variables, for which NJETS does not provide the full distributions, are excluded. . . . . . . . . . . . . . . . . . 8 
6.3 Statistical comparison of agreement between observed and predicted distributions of the four-jet variables. The $\chi^{2}$ s per degree of freedom are listed. In calculating the over all $\chi^{2} s$, the single body mass fraction variables, for which NJETS does not provide the full distributions, are

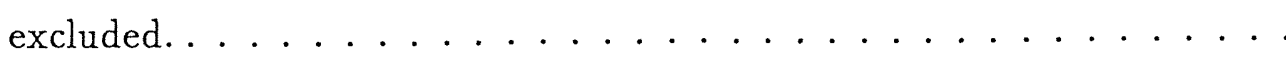

6.4 Statistical comparison of agreement between observed and predicted distributions of the five-jet variables. The $\chi^{2}$ s per degree of freedom are listed. In calculating the over all $\chi^{2}$ s, the single body mass fraction variables, for which NJETS does not provide the full distributions, are excluded. . . . . . . . . . . . . . . . .

6.5 The observed and predicted cross-sections for inclusive three-jet, fourjet, and five-jet events. Errors are statistical only. . . . . . . . . .

7.1 Parameter choices used for the eight NJETS calculations.

7.2 Statistical comparison of agreement between observed 3-jet distributions and distributions predicted by NJETS for eight parameter choices. The $\chi^{2}$ s per degree of freedom are listed. . . . . . . . . . . . .

7.3 Statistical comparison of agreement between observed 4-jet distributions and distributions predicted by NJETS for eight parameter choices. The $\chi^{2}$ s per degree of freedom are listed. . . . . . . . . . . . .

7.4 Statistical comparison of agreement between NJETS predictions and observed distributions of 3 -jet events in three different luminosity ranges. low: $\mathcal{L}<4 \times 10^{30} \mathrm{~cm}^{-2} \mathrm{~s}^{-1}$, mid: $4<\mathcal{L}<8 \times 10^{30} \mathrm{~cm}^{-2} \mathrm{~s}^{-1}$, and high: $\mathcal{L}>8 \times 10^{30} \mathrm{~cm}^{-2} \mathrm{~s}^{-1}$ respectively. The $\chi^{2}$ s per degree of freedom are listed.

7.5 Statistical comparison of agreement between NJETS predictions and observed distributions of 4-jet events in three different luminosity ranges. low: $\mathcal{L}<4 \times 10^{30} \mathrm{~cm}^{-2} \mathrm{~s}^{-1}$, mid: $4<\mathcal{L}<8 \times 10^{30} \mathrm{~cm}^{-2} \mathrm{~s}^{-1}$, and high: $\mathcal{L}>8 \times 10^{30} \mathrm{~cm}^{-2} \mathrm{~s}^{-1}$ respectively. The $\chi^{2}$ s per degree of freedom are listed.

7.6 Statistical comparison of agreement between NJETS predictions and observed distributions of 5 -jet events in three different luminosity ranges. low: $\mathcal{L}<4 \times 10^{30} \mathrm{~cm}^{-2} \mathrm{~s}^{-1}$, mid: $4<\mathcal{L}<8 \times 10^{30} \mathrm{~cm}^{-2} \mathrm{~s}^{-1}$, and high: $\mathcal{L}>8 \times 10^{30} \mathrm{~cm}^{-2} \mathrm{~s}^{-1}$ respectively. The $\chi^{2}$ 's per degree of freedom are listed. 
7.7 Statistical comparison of agreement between NJETS predictions and observed distributions of 3 -jet events with energies shifted by $-7 \%, 0 \%$, and $+7 \%$. The $\chi^{2}$ s per degree of freedom are listed. . . . . . . . 112

7.8 Statistical comparison of agreement between NJETS predictions and observed distributions of 4 -jet events with energies shifted by $-7 \%, 0 \%$, and $+7 \%$. The $\chi^{2}$ s per degree of freedom are listed. . . . . . . . . 113

7.9 Statistical comparison of agreement between NJETS predictions and observed distributions of 5 -jet events with energies shifted by $-7 \%, 0 \%$, and $+7 \%$. The $\chi^{2}$ s per degree of freedom are listed. . . . . . . . 113

A.1 Parameters of three-jet events shown in Figure A.1. . . . . . . . . . 150

A.2 Parameters of four-jet events shown in Figure A.2 . . . . . . . . 150

A.3 Parameters of five-jet events shown in Figure A.3 . . . . . . . . . . 151 


\section{The CDF Collaboration}

F. Abe, ${ }^{14}$ H. Akimoto, ${ }^{32}$ A. Akopian, ${ }^{27}$ M. G. Albrow, ${ }^{7}$ S. R. Amendolia ${ }^{23}$ D. Amidei, ${ }^{17}$ J. Antos, ${ }^{29}$ C. Anway-Wiese,${ }^{4}$ S. Aota, ${ }^{32}$ G. Apollinari, ${ }^{27}$ T. Asakawa ${ }^{32}$ W. Ashmanskas, ${ }^{15}$ M. Atac,${ }^{7}$ P. Auchincloss, ${ }^{26}$ F. Azfar, ${ }^{22}$ P. Azzi-Bacchetta ${ }^{21}$ N. Bacchetta, ${ }^{21}$ W. Badgett, ${ }^{17}$ S. Bagdasarov, ${ }^{27}$ M. W. Bailey, ${ }^{19}$ J. Bao, ${ }^{35}$ P. de Barbaro, ${ }^{26}$ A. Barbaro-Galtieri, ${ }^{15}$ V. E. Barnes, ${ }^{25}$ B. A. Barnett, ${ }^{13}$ E. Barz i, G. Bauer,${ }^{16}$ T. Baumann, ${ }^{9}$ F. Bedeschi, ${ }^{23}$ S. Behrends ${ }^{3}$ S. Belforte, ${ }^{23}$ G. Bellettini,${ }^{23}$ J. Bellinger, ${ }^{34}$ D. Benjamin, ${ }^{31}$ J. Benlloch, ${ }^{16}$ J. Bensinger, ${ }^{3}$ D. Benton, ${ }^{22}$ A. Beretva: ${ }^{3}{ }^{7}$ J. P. Berge ${ }^{7}$ J. Berryhill, ${ }^{5}$ S. Bertolucci, ${ }^{8}$ A. Bhatti, ${ }^{27}$ K. Biery, ${ }^{12}$ M. Binkler ${ }^{\gamma}{ }^{7}$ D. Bisello, ${ }^{21}$ R. E. Blair, ${ }^{1}$ C. Blocker ${ }^{3}$ A. Bodek, ${ }^{26}$ W. Bokhari, ${ }^{16}$ V. Bolognes i, ${ }^{7}$ D. Bortoletto, ${ }^{25}$ J. Boudreau, ${ }^{24}$ L. Breccia, ${ }^{2}$ C. Bromberg, ${ }^{18}$ N. Bruner, ${ }^{19}$ E. Buckle $\mathrm{y}-$ Geer, ${ }^{7}$ H. S. Budd, ${ }^{26}$ K. Burkett, ${ }^{17}$ G. Busetto, ${ }^{21}$ A. Byon-Wagner,${ }^{7}$ K. L. Byrum $1,{ }^{1}$ J. Cammerata, ${ }^{13}$ C. Campagnari, ${ }^{7}$ M. Campbell, ${ }^{17}$ A. Caner,${ }^{7}$ W. Carithers: ${ }^{15}$ D. Carlsmith, ${ }^{34}$ A. Castro, ${ }^{21}$ D. Cauz, ${ }^{23}$ Y. Cen, ${ }^{26}$ F. Cervelli, ${ }^{23}$ H. Y. Chao, ${ }^{29}$ J. Chapman, ${ }^{17}$ M.-T. Cheng, ${ }^{29}$ G. Chiarelli, ${ }^{23}$ T. Chikamatsu, ${ }^{32}$ C. N. Chiou, ${ }^{29}$ L. Christofek, ${ }^{11}$ S. Cihangir, ${ }^{7}$ A. G. Clark, ${ }^{23}$ M. Cobal, ${ }^{23}$ M. Contreras, ${ }^{5}$ J. Conway: ${ }^{28}$ J. Cooper ${ }^{7}$ M. Cordelli, ${ }^{8}$ C. Couyoumtzelis, ${ }^{23}$ D. Crane, ${ }^{1}$ D. Cronin-Hennessy $r,{ }^{6}$ R. Culbertson, ${ }^{5}$ J. D. Cunningham, ${ }^{3}$ T. Daniels,${ }^{16}$ F. DeJongh, ${ }^{7}$ S. Delchamps $;{ }^{7}$ S. Dell'Agnello, ${ }^{23}$ M. Dell'Orso, ${ }^{23}$ L. Demortier, ${ }^{27}$ B. Denby, ${ }^{23}$ M. Deninnc ${ }^{2}{ }^{2}$ P. F. Derwent ${ }^{17}$ T. Devlin, ${ }^{28}$ M. Dickson, ${ }^{26}$ J. R. Dittmann, ${ }^{6}$ S. Donati, ${ }^{23}$ J. Done,,${ }^{30}$ T. Dorigo, ${ }^{21}$ A. Dunn, ${ }^{17}$ N. Eddy ${ }^{17}$ K. Einsweiler ${ }^{15}$ J. E. Elias, ${ }^{7}$ R. Ely, ${ }^{15}$ E. E ngels, Jr. ${ }^{24}$ D. Errede, ${ }^{11}$ S. Errede ${ }^{11}$ Q. Fan,${ }^{26}$ I. Fiori, ${ }^{2}$ B. Flaugher,${ }^{7}$ G. W. Foster $:{ }^{7}$ M. Franklin, ${ }^{9}$ M. Frautschi, ${ }^{31}$ J. Freeman, ${ }^{7}$ J. Friedman, ${ }^{16}$ H. Frisch, ${ }^{5}$ T. A. Fuess $;{ }^{1}$ Y. Fukui, ${ }^{14}$ S. Funaki, ${ }^{32}$ G. Gagliardi, ${ }^{23}$ S. Galeotti, ${ }^{23}$ M. Gallinaro, ${ }^{21}$ M. Garci aSciveres ${ }^{15}$ A. F. Garfinkel, ${ }^{25}$ C. Gay, ${ }^{9}$ S. Geer,${ }^{7}$ D. W. Gerdes, ${ }^{17}$ P. Giannetti, ${ }^{23}$ N. Giokaris, ${ }^{27}$ P. Giromini, ${ }^{8}$ L. Gladney, ${ }^{22}$ D. Glenzinski, ${ }^{13}$ M. Gold, ${ }^{19}$ J. Gonzalez, ${ }^{22}$ A. Gordon, ${ }^{9}$ A. T. Goshaw, ${ }^{6}$ K. Goulianos, ${ }^{27}$ H. Grassmann, ${ }^{23}$ L. Groer,${ }^{28}$ C. Gross oPilcher, ${ }^{5}$ G. Guillian, ${ }^{17}$ R. S. Guo, ${ }^{29}$ C. Haber, ${ }^{15}$ E. Hafen, ${ }^{16}$ S. R. Hahn, ${ }^{7}$ R. Hamilton ${ }^{9}$, R. Handler, ${ }^{34}$ R. M. Hans, ${ }^{35}$ K. Hara, ${ }^{32}$ A. D. Hardman, ${ }^{25}$ B. Harral, ${ }^{22}$ R. M. Harris ; ${ }^{7}$ S. A. Hauger, ${ }^{6}$ J. Hauser ${ }^{4}$ C. Hawk, ${ }^{28}$ E. Hayashi, ${ }^{32}$ J. Heinrich, ${ }^{22}$ K. D. Hoffman, ${ }^{25}$ M. Hohlmann, ${ }^{1,5}$ C. Holck, ${ }^{22}$ R. Hollebeek, ${ }^{22}$ L. Holloway, ${ }^{11}$ A. Hölscher, ${ }^{12}$ S. Hong, ${ }^{17}$ 
G. Houk, ${ }^{22}$ P. Hu, ${ }^{24}$ B. T. Huffman, ${ }^{24}$ R. Hughes,${ }^{26}$ J. Huston, ${ }^{18}$ J. Huth, ${ }^{9}$ J. Hylen, ${ }^{7}$ H. Ikeda, ${ }^{32} \mathrm{M}$. Incagli, ${ }^{23} \mathrm{~J}$. Incandela,${ }^{7} \mathrm{G}$. Introzzi, ${ }^{23} \mathrm{~J}$. Iwai, ${ }^{32} \mathrm{Y}$. Iwata, ${ }^{10} \mathrm{H}$. Jensen, ${ }^{7}$ U. Joshi, ${ }^{7}$ R. W. Kadel, ${ }^{15}$ E. Kajfasz ${ }^{7 a}$ T. Kamon, ${ }^{30}$ T. Kaneko, ${ }^{32}$ K. Karr, ${ }^{33}$ H. Kasha, ${ }^{35}$ Y. Kato, ${ }^{20}$ T. A. Keaffaber ${ }^{25}$ L. Keeble,${ }^{8}$ K. Kelley, ${ }^{16}$ R. D. Kennedy, ${ }^{28}$ R. Kephart,${ }^{7}$ P. Kesten, ${ }^{15}$ D. Kestenbaum, ${ }^{9}$ R. M. Keup,${ }^{11}$ H. Keutelian, ${ }^{7}$ F. Keyvan,${ }^{4}$ B. Kharadia, ${ }^{11}$ B. J. Kim, ${ }^{26}$ D. H. Kim, ${ }^{7 a}$ H. S. Kim, ${ }^{12}$ S. B. Kim,${ }^{17}$ S. H. Kim, ${ }^{32}$ Y. K. Kim, ${ }^{15}$ L. Kirsch, ${ }^{3}$ P. Koehn, ${ }^{26}$ K. Kondo, ${ }^{32}$ J. Konigsberg, ${ }^{9}$ S. Kopp ${ }^{5}$ K. Kordas, ${ }^{12}$ W. Koska, ${ }^{7}$ E. Kovacs, ${ }^{7 a}$ W. Kowald, ${ }^{6}$ M. Krasberg, ${ }^{17}$ J. Kroll, ${ }^{7}$ M. Kruse, ${ }^{25}$ T. Kuwabara, ${ }^{32}$ S. E. Kuhlmann, ${ }^{1}$ E. Kuns, ${ }^{28}$ A. T. Laasanen, ${ }^{25}$ N. Labanca, ${ }^{23}$ S. Lammel, ${ }^{7}$ J. I. Lamoureux, ${ }^{3}$ T. LeCompte, ${ }^{11}$ S. Leone, ${ }^{23}$ J. D. Lewis ${ }^{7}$ P. Limon, ${ }^{7}$ M. Lindgren, ${ }^{4}$ T. M. Liss,${ }^{11}$ N. Lockyer, ${ }^{22}$ O. Long, ${ }^{22}$ C. Loomis ${ }^{28}$ M. Loreti, ${ }^{21} \mathrm{~J} . \mathrm{Lu},{ }^{30}$ D. Lucchesi,${ }^{23}$ P. Lukens ${ }^{7}$ S. Lusin,${ }^{34} \mathrm{~J}$. Lys,${ }^{15} \mathrm{~K}$. Maeshima,${ }^{7}$ A. Maghakian, ${ }^{27}$ P. Maksimovic, ${ }^{16}$ M. Mangano, ${ }^{23}$ J. Mansour, ${ }^{18}$ M. Mariotti, ${ }^{21}$ J. P. Marriner, ${ }^{7}$ A. Martin, ${ }^{11}$ J. A. J. Matthews ${ }^{19}$ R. Mattingly, ${ }^{16}$ P. McIntyre, ${ }^{30}$ P. Melese, ${ }^{27}$ A. Menzione, ${ }^{23}$ E. Meschi, ${ }^{23}$ S. Metzler, ${ }^{22}$ C. Miao, ${ }^{17}$ G. Michail ${ }^{9}$ R. Miller ${ }^{18} \mathrm{H}$. Minato, ${ }^{32}$ S. Miscetti, ${ }^{8}$ M. Mishina, ${ }^{14} \mathrm{H}$. Mitsushio, ${ }^{32}$ T. Miyamoto ${ }^{32}$ S. Miyashita, ${ }^{32}$ Y. Morita, ${ }^{14}$ J. Mueller,${ }^{24}$ A. Mukherjee, ${ }^{7}$ T. Muller,${ }^{4}$ P. Murat,${ }^{23}$ H. Nakada, ${ }^{32}$ I. Nakano, ${ }^{32}$ C. Nelson, ${ }^{7}$ D. Neuberger ${ }^{4}$ C. Newman-Holmes, ${ }^{7}$ M. Ninomiya, ${ }^{32}$ L. Nodulman, ${ }^{1}$ S. H. Oh, ${ }^{6}$ K. E. Ohl, ${ }^{35}$ T. Ohmoto, ${ }^{10}$ T. Ohsugi, ${ }^{10}$ R. Oishi, ${ }^{32}$ M. Okabe, ${ }^{32}$ T. Okusawa, ${ }^{20}$ R. Oliver, ${ }^{22}$ J. Olsen, ${ }^{34}$ C. Pagliarone, ${ }^{2}$ R. Paoletti ${ }^{23}$ V. Papadimitriou, ${ }^{31}$ S. P. Pappas ${ }^{35}$ S. Park, ${ }^{7}$ A. Parri, ${ }^{8}$ J. Patrick, ${ }^{7}$ G. Pauletta, ${ }^{23}$ M. Paulini, ${ }^{15}$ A. Perazzo,${ }^{23}$ L. Pescara, ${ }^{21}$ M. D. Peters, ${ }^{15}$ T. J. Phillips, ${ }^{6}$ G. Piacentino, ${ }^{2}$ M. Pillai, ${ }^{26}$ K. T. Pitts,${ }^{7}$ R. Plunkett,${ }^{7}$ L. Pondrom,${ }^{34}$ J. Proudfoot ${ }^{1}$ F. Ptohos, ${ }^{9}$ G. Punzi, ${ }^{23}$ K. Ragan, ${ }^{12}$ A. Ribon, ${ }^{21}$ F. Rimondi, ${ }^{2}$ L. Ristori, ${ }^{23}$ W. J. Robertson, ${ }^{6}$ T. Rodrigo, ${ }^{7 a}$ S. Rolli, ${ }^{23}$ J. Romano, ${ }^{5}$ L. Rosenson, ${ }^{16}$ R. Roser,${ }^{11}$ W. K. Sakumoto, ${ }^{26}$ D. Saltzberg, ${ }^{5}$ A. Sansoni, ${ }^{8}$ L. Santi, ${ }^{23}$ H. Sato, ${ }^{32}$ V. Scarpine, ${ }^{30}$ P. Schlabach, ${ }^{9}$ E. E. Schmidt, ${ }^{7}$ M. P. Schmidt, ${ }^{35}$ A. Scribano, ${ }^{23}$ S. Segler, ${ }^{7}$ S. Seidel, ${ }^{19}$ Y. Seiya, ${ }^{32}$ G. Sganos, ${ }^{12}$ A. Sgolacchia, ${ }^{2}$ M. D. Shapiro, ${ }^{15}$ N. M. Shaw ${ }^{25}$ Q. Shen, ${ }^{25}$ P. F. Shepard, ${ }^{24}$ M. Shimojima, ${ }^{32}$ M. Shochet,${ }^{5}$ J. Siegrist, ${ }^{15}$ A. Sill, ${ }^{31}$ P. Sinervo, ${ }^{12}$ P. Singh, ${ }^{24}$ J. Skarha, ${ }^{13}$ K. Sliwa, ${ }^{33}$ F. D. Snider, ${ }^{13}$ T. Song, ${ }^{17}$ J. Spalding, ${ }^{7}$ P. Sphicas, ${ }^{16}$ F. Spinella, ${ }^{23}$ M. Spiropulu, ${ }^{9}$ L. Spiegel, ${ }^{7}$ L. Stanco, ${ }^{21}$ J. Steele, ${ }^{34}$ A. Stefanini, ${ }^{23}$ K. Strahl, ${ }^{12}$ J. Strait, ${ }^{7}$ R. Ströhmer, ${ }^{9}$ D. Stuart, ${ }^{7}$ G. Sullivan, ${ }^{5}$ A. Soumarokov, ${ }^{29}$ K. Sumorok, ${ }^{16}$ J. Suzuki, ${ }^{32}$ T. Takada, ${ }^{32}$ T. Takahashi, ${ }^{20}$ T. Takano, ${ }^{32}$ K. Takikawa, ${ }^{32}$ N. Tamura, ${ }^{10}$ F. Tartarelli, ${ }^{23}$ W. Taylor, ${ }^{12}$ P. K. Teng, ${ }^{29}$ Y. Teramoto, ${ }^{20}$ 
S. Tether ${ }^{16}$ D. Theriot, ${ }^{7}$ T. L. Thomas, ${ }^{19}$ R. Thun, ${ }^{17}$ M. Timko, ${ }^{33}$ P. Tipton, ${ }^{26}$ A. Titov, ${ }^{27}$ S. Tkaczyk, ${ }^{7}$ D. Toback, ${ }^{5}$ K. Tollefson, ${ }^{26}$ A. Tollestrup, ${ }^{7}$ J. Tonnison, ${ }^{25}$ J. F. de Troconiz, ${ }^{9}$ S. Truitt, ${ }^{17}$ J. Tseng, ${ }^{13}$ N. Turini, ${ }^{23}$ T. Uchida, ${ }^{32}$ N. Uemura, ${ }^{32}$ F. Ukegawa, ${ }^{22}$ G. Unal, ${ }^{22}$ S. C. van den Brink, ${ }^{24}$ S. Vejcik, III, ${ }^{17}$ G. Velev, ${ }^{23}$ R. Vidal, ${ }^{7}$ M. Vondracek, ${ }^{11}$ D. Vucinic, ${ }^{16}$ R. G. Wagner, ${ }^{1}$ R. L. Wagner, ${ }^{7}$ J. Wahl, ${ }^{5}$ C. Wang, ${ }^{6}$ C. H. Wang, ${ }^{29}$ G. Wang, ${ }^{23}$ J. Wang, ${ }^{5}$ M. J. Wang, ${ }^{29}$ Q. F. Wang, ${ }^{27}$ A. Warburton, ${ }^{12}$ G. Watts,${ }^{26}$ T. Watts,${ }^{28}$ R. Webb,${ }^{30}$ C. Wei, ${ }^{6}$ C. Wendt,${ }^{34}$ H. Wenzel,${ }^{15}$ W. C. Wester, III, ${ }^{7}$ A. B. Wicklund, ${ }^{1}$ E. Wicklund, ${ }^{7}$ R. Wilkinson, ${ }^{22}$ H. H. Williams, ${ }^{22}$ P. Wilson, ${ }^{5}$ B. L. Winer,${ }^{26}$ D. Wolinski, ${ }^{17} \mathrm{~J}$. Wolinski, ${ }^{18} \mathrm{X} . \mathrm{Wu},{ }^{23} \mathrm{~J}$. Wyss ${ }^{21}$ A. Yagil, ${ }^{7}$ W. Yao, ${ }^{15}$ K. Yasuoka, ${ }^{32}$ Y. Ye, ${ }^{12}$ G. P. Yeh, ${ }^{7}$ P. Yeh, ${ }^{29}$ M. Yin, ${ }^{6}$ J. Yoh, ${ }^{7}$ C. Yosef,,${ }^{18}$ T. Yoshida, ${ }^{20}$ D. Yovanovitch, ${ }^{7}$ I. Yu, ${ }^{35}$ L. Yu, ${ }^{19}$ J. C. Yun,${ }^{7}$ A. Zanetti, ${ }^{23}$ F. Zetti, ${ }^{23}$ L. Zhang, ${ }^{34}$ W. Zhang, ${ }^{22}$ and S. Zucchelli ${ }^{2}$

(CDF Collaboration)

1 Argonne National Laboratory, Argonne, Illinois 60439

2 Istituto Nazionale di Fisica Nucleare, University of Bologna, I-40126 Bologna, Italy

3 Brandeis University, Waltham, Massachusetts 02254

4 University of California at Los Angeles, Los Angeles, California 90024

5 University of Chicago, Chicago, Illinois 60637

6 Duke University, Durham, North Carolina 27708

7 Fermi National Accelerator Laboratory, Batavia, Illinois 60510

8 Laboratori Nazionali di Frascati, Istituto Nazionale di Fisica Nucleare, I-00044 Frascati, Italy

9 Harvard University, Cambridge, Massachusetts 02138

10 Hiroshima University, Higashi-Hiroshima 724, Japan

11 University of Illinois, Urbana, Illinois 61801

12 Institute of Particle Physics, McGill University, Montreal H3A 2T8, and University of Toronto,

Toronto M5S 1A7, Canada

13 The Johns Hopkins University, Baltimore, Maryland 21218

14 National Laboratory for High Energy Physics (KEK), Tsukuba, Ibaraki 305, Japan

15 Lawrence Berkeley Laboratory, Berkeley, California 94720

16

Massachusetts Institute of Technology, Cambridge, Massachusetts 02139

17 University of Michigan, Ann Arbor, Michigan 48109

18 Michigan State University, East Lansing, Michigan 48824 
19 University of New Mexico, Albuquerque, New Mexico 87131

20 Osaka City University, Osaka 588, Japan

21 Universita di Padova, Istituto Nazionale di Fisica Nucleare, Sezione di Padova, I-35131 Padova, Italy

22 University of Pennsylvania, Philadelphia, Pennsylvania 19104

23 Istituto Nazionale di Fisica Nucleare, University and Scuola Normale Superiore of Pisa, I-56100 Pisa, Italy

24 University of Pittsburgh, Pittsburgh, Pennsylvania 15260

25 Purdue University, West Lafayette, Indiana 47907

26 University of Rochester, Rochester, New York 14627

27 Rockefeller University, New York, New York 10021

28

Rutgers University, Piscataway, New Jersey 08854

29 Academia Sinica, Taipei, Taiwan 11529, Republic of China

30 Texas A $8 M$ University, College Station, Texas 77843

31 Texas Tech University, Lubbock, Texas 79409

32 University of Tsukuba, Tsukuba, Ibaraki 305, Japan

33 Tufts University, Medford, Massachusetts 02155

34 University of Wisconsin, Madison, Wisconsin 53706

35 Yale University, New Haven, Connecticut 06511 


\section{Chapter 1}

\section{Introduction}

The Fermilab Tevatron Proton-Antiproton Collider provides a unique opportunity to study the properties of strong interactions in $\bar{p} p$ collisions at short distance. Large samples of events containing two or more jets have recently been collected with the Collider Detector at Fermilab (CDF). Many of the observed events contain three-, four, or even five-or-more jets [1]. A comprehensive analysis of these multijet events would provide an interesting test of the QCD calculations. In particular the analysis can provide a test of leading order (LO) perturbative Quantum Chromodynamics (QCD) $2 \rightarrow N$ calculations. In the last few years complete LO QCD matrix elements have become available for $N=3[2], N=4$ [3], and $N=5$ [4]. Partial calculations exist for $N>5$ [5]. The $2 \rightarrow N$ calculations are complicated, and have required the development of new techniques [6]. Unfortunately the computing resources needed to evaluate the matrix elements increase rapidly with $\mathrm{N}$. A considerable effort has therefore been devoted to finding approximations to the exact LO matrix elements that permit faster calculations [7]. A comprehensive analysis of multijet events at high energy hadron colliders can provide a test of any approximations (e.g. parton shower model) that may be used in present or future $2 \rightarrow N$ calculations. Finally, in addition to providing a test of the QCD calculations, a detailed understanding of the properties of multijet events produced in high energy hadron-hadron collisions is important because multijet production is expected to be prolific in future high luminosity running at the Fermilab Proton-Antiproton Collider and at the Large Hadron Collider at CERN. A comprehensive understanding of QCD multijet production is therefore required to 
facilitate searches for more exotic processes producing multijet events. For example, the properties of six-jet events at the Fermilab Collider are likely to be important in the near future for the study of top-quark pair production and their decays in the all hadronic channel.

\subsection{Quantum Chromodynamics}

Quantum Chromodynamics (QCD) is regarded as the best viable theory of the strong interactions. Recent theoretical and experimental developments have significantly increased our ability to test QCD and have deepened our understanding of hadronic interactions. This is particularly true for higher energy processes where the decreasing value of the coupling constant $\alpha_{s}(\mu)$ allows reliable results from a perturbative expansion.

Quarks and gluons alone experience and transmit strong forces. A theoretical concept called "color" is required to describe these forces. Historically, color was introduced to explain the existence of the $\Delta^{++}$baryon which is composed of three $u$ quarks and has $J^{P}=\frac{3}{2}^{+}$, hence the spin assignments of the quarks must be $(u \uparrow, u \uparrow, u \uparrow)$ which clearly violates the Pauli principle. To overcome this difficulty in the theory of QCD each quark carries a color charge that can take on three values; $R=$ red, $G=$ green, and $B=$ blue, and each antiquark carries an anti-color charge; $\bar{R}, \bar{G}$, and $\bar{B}$. Then the quark assignments for the $\Delta^{++}$become $\left(u_{R} \uparrow, u_{G} \uparrow, u_{B} \uparrow\right)$ and the Pauli principle is no longer violated. The field quantum (gluon) carries two labels of colors (one color and one anti-color) such that color is conserved at each quark-quark-gluon vertex. When the gluon is emitted, the quark color changes giving eight ways of coupling a gluon between an initial and a final quark. The strong forces transmitted by gluons differ significantly from the electromagnetic forces transmitted by photons, because gluons carry color. For example, gluons can couple directly to other gluons whereas photons cannot couple directly to photons. The most striking consequence is color confinement; experiment and theory both suggest that only colorless states are allowed as physical hadrons. This means that neither quarks nor gluons can appear in isolation; they can only exist within colorless composite hadrons. In electromagnetic terms, this is like 
allowing only neutral atoms to exist and forbidding all ionization. The strong forces between these hadrons are then like the Van der Waals forces between electrically neutral atoms, which are suppressed at large distance.

The strength of the strong interaction between the quarks is characterized by the strong coupling constant $\alpha_{s}$. In fact this coupling is not a constant but actually depends on the momentum transfer squared $Q^{2}$ in the particular process. In a model with $n_{f}$ quark flavors, $\alpha_{s}\left(Q^{2}\right)$ is written as

$$
\alpha_{s}\left(Q^{2}\right)=\frac{\alpha_{s}\left(\mu^{2}\right)}{1+B \alpha_{s}(\mu) \log \left(Q^{2} / \mu^{2}\right)}=\frac{1}{1+B \log \left(Q^{2} / \Lambda^{2}\right)},
$$

where $B=\left(33-2 n_{f}\right) / 12 \pi, n_{f}$ is a number of quark flavors, and $\Lambda$ is a scale parameter given by

$$
\Lambda^{2}=\mu^{2} \exp \left(\frac{-1}{B \alpha_{a}\left(\mu^{2}\right)}\right) .
$$

The function (1.1) suggests that the perturbation theory for QCD should work well at high $Q^{2}$ (short distance) where $\alpha_{s}$ becomes small. The property, $\alpha_{s} \rightarrow 0$ for $Q^{2} \rightarrow \infty$, is called "asymptotic freedom". The perturbation theory breaks down at small $Q^{2}$ (large distance) where $\alpha_{s}$ becomes large. The property, $\alpha_{s} \rightarrow$ large for $Q^{2} \rightarrow$ small, suggests that quarks are confined within hadrons. The constant $\Lambda$ characterizes the scale of $Q^{2}$ at which perturbation theory breaks down. The characteristic of asymptotic freedom is opposite to the effect that occurs in Quantum Electrodynamics (QED) where $\alpha$ decreases as $Q^{2}$ increases.

Neither quarks nor gluons can exist in isolation. They are usually observed as "jets", a collection of many observable (colorless) hadrons, through the hadronization process. The four momentum of a parent parton is approximately equal to one in the observed jet in high energy collisions, hence one can survey the QCD parton-level processes through the study of jet production. 


\subsection{Theoretical Models for Multijet Production}

The cross-section for the production of the $\mathrm{N}$-parton final state, $1+2 \rightarrow 3+4+\cdots+$ $(\mathrm{N}+2)$, in $p \bar{p}$ collisions is described by the expression:

$$
\sigma_{N}=\sum_{l} \int f_{1}^{l}\left(x_{1}\right) f_{2}^{l}\left(x_{2}\right)\left|\mathcal{M}_{l}^{N}\right|^{2} \Phi_{N} d x_{1} d x_{2}
$$

where the sum is over all possible $1+2 \rightarrow N$ subprocesses. The functions $f_{1}^{l}\left(x_{1}\right)$ and $f_{2}^{l}\left(x_{2}\right)$ are the parton density (structure) functions of the incoming partons, $\left|\mathcal{M}_{l}^{N}\right|^{2}$ represents the matrix elements of the subprocesses, and $\Phi_{N}$ is the $N$-body phase-space.

The number of subprocesses increases rapidly with N. In Figures 1.1, 1.2, and 1.3 shown are small subsets of the allowed Feynman diagrams for three-parton final states (at $\mathcal{O}\left(\alpha_{s}^{3}\right)$ ), four-parton final states $\left(\right.$ at $\left.\mathcal{O}\left(\alpha_{s}^{4}\right)\right)$, and five-parton final states (at $\mathcal{O}\left(\alpha_{s}^{5}\right)$ ) respectively.

The explicit forms of $|\mathcal{M}|^{2}$ for processes for parton-parton scattering are given in Table 1.1 as a function of the Mandelstam variables:

$$
\begin{aligned}
& \hat{s}=\left(p_{1}+p_{2}\right)^{2} \\
& \hat{t}=\left(p_{1}-p_{3}\right)^{2}=-\frac{\hat{s}}{2}(1-\cos \theta) \\
& \hat{u}=\left(p_{1}-p_{4}\right)^{2}=-\frac{\hat{s}}{2}(1+\cos \theta)
\end{aligned}
$$

where $p_{1}$ and $p_{2}$ are the incoming parton four momenta, $p_{3}$ and $p_{4}$ are the outgoing parton momenta, $\hat{s}$ corresponds to the center-of-mass energy squared, and $\theta$ is the scattering angle in the center-of-mass system.

There presently exist two approaches for modeling perturbative QCD for multijet productions. The first is the matrix element method, in which Feynman diagrams are calculated order-by-order in $\alpha_{s}$. The second approach is based on a parton shower scheme. Both methods will be briefly described in Chapter 4.

\subsection{Analysis Overview}

In the past, elegant analyses of two-jet and three-jet production have been carried out by the UA1 $[8,9,10]$ and UA2 $[11,12]$ collaborations (1982-1991) at the CERN $S \bar{p} p S$ 


\begin{tabular}{|c|c|c|c|}
\hline \multirow{2}{*}{$\frac{\text { Parton }}{q_{1} q_{2}}$} & \multicolumn{2}{|c|}{ Subprocess } & $|\mathcal{M}|^{2}$ \\
\hline & $\rightarrow$ & $q_{1} q_{2}$ & $4 \hat{s}^{2}+\hat{u}^{2}$ \\
\hline$q_{1} \bar{q}_{2}$ & $\rightarrow$ & $q_{1} \bar{q}_{2}$ & $\overline{9} \hat{t}^{2}$ \\
\hline$q_{1} q_{1}$ & $\rightarrow$ & $q_{1} q_{1}$ & $\frac{4}{9}\left(\frac{\hat{s}^{2}+\hat{u}^{2}}{\hat{t}^{2}}+\frac{\hat{s}^{2}+\hat{t}^{2}}{\hat{u}^{2}}\right)-\frac{8}{27} \frac{\hat{s}^{2}}{\hat{u} \hat{t}}$ \\
\hline$q_{1} \bar{q}_{1}$ & $\rightarrow$ & $q_{2} \bar{q}_{2}$ & $\frac{4}{9} \frac{\hat{t}^{2}+\hat{u}^{2}}{\hat{s}^{2}}$ \\
\hline$q_{1} \bar{q}_{1}$ & $\rightarrow$ & $q_{1} \bar{q}_{1}$ & $\frac{4}{9}\left(\frac{\hat{s}^{2}+\hat{u}^{2}}{\hat{t}^{2}}+\frac{\hat{t}^{2}+\hat{u}^{2}}{\hat{s}^{2}}\right)-\frac{8}{27} \frac{\hat{u}^{2}}{\hat{s} \hat{t}}$ \\
\hline$q \bar{q}$ & $\rightarrow$ & $g g$ & $\frac{32}{27} \frac{\hat{u}^{2}+\hat{t}^{2}}{\hat{u} \hat{t}}-\frac{8}{3} \frac{\hat{u}^{2}+\hat{t}^{2}}{\hat{s}^{2}}$ \\
\hline$g g$ & $\rightarrow$ & $q \bar{q}$ & $\frac{1}{6} \frac{\hat{u}^{2}+\hat{t}^{2}}{\hat{u} \hat{t}}-\frac{8}{3} \frac{\hat{u}^{2}+\hat{t}^{2}}{\hat{s}^{2}}$ \\
\hline$q g$ & $\rightarrow$ & $q g$ & $-\frac{4}{9} \frac{\hat{u}^{2}+\hat{s}^{2}}{\hat{u} \hat{s}}+\frac{\hat{u}^{2}+\hat{s}^{2}}{\hat{t}^{2}}$ \\
\hline$g g$ & $\rightarrow$ & $g g$ & $-\frac{9}{2}\left(\frac{\hat{u} \hat{t}}{\hat{s}^{2}}+\frac{\hat{u} \hat{s}}{\hat{t}^{2}}+\frac{\hat{s} \hat{t}}{\hat{u}^{2}}\right)+\frac{27}{2}$ \\
\hline
\end{tabular}

Table 1.1: Lowest order parton-parton cross-sections.

Collider and by the CDF (1989-1993) [13, 14] and D0 (1995) [15] collaborations at the Fermilab Tevatron Collider. These studies suggested that QCD predictions give a reasonable description of two-jet and three-jet production in hadron-hadron collisions.

There have also been analyses of events with more than three jets $[15,16,17]$. However, the analyses of events with four or more jets have not used a simple set of independent variables that (i) span the multijet parameter space, (ii) make it simple to interpret the observed distributions within the framework of perturbative QCD, and (iii) make it easy to compare the characteristics of events having $N$ jets with the characteristics of events having for example $(N+1)$ jets. 
In our analysis observed jets are thought of as massive objects. In choosing a set of multijet variables that span the multijet parameter space it should be noted that we can completely define a system of $\mathrm{N}$ massive bodies in the $\mathrm{N}$-body rest-frame by specifying the $4 \mathrm{~N}$ components of four-momentum. The $\mathrm{N}$-body system would then be overspecified since momentum conservation provides us with three constraints. Furthermore, we can rotate the $\mathrm{N}$-body system about the incoming beam direction without losing any information. Therefore, to describe the system we need only specify ( $4 \mathrm{~N}-4)$ parameters. We will take these parameters to be the $\mathrm{N}$-body mass and $(4 \mathrm{~N}-5)$ additional variables. We therefore introduce and discuss a set of $(4 \mathrm{~N}-5)$ dimensionless variables which, with the addition of the multijet mass, span the multijet parameter space. Our $(4 \mathrm{~N}-5)$ multijet variables will provide a simple framework within which the properties of observed multijet events can be compared with QCD predictions. To illustrate the use of our multijet variables we compare the predictions from exact LO QCD matrix element calculations with the corresponding predictions from a QCD parton shower Monte Carlo program, and from a model in which the events are uniformly distributed over the available $\mathrm{N}$-body phase-space.

In Chapter 2 we briefly summarize the apparatus including the Tevatron collider and the major components of the detectors. Both QCD predictions, (1) the exact LO matrix element calculations and (2) the parton shower calculations, and phase-space model predictions used in this analysis are described in Chapter 4. In Chapter 5 we introduce our multijet variables; the standard three-jet variables are reviewed and extended, and then four-jet and five-jet variables are introduced and discussed. The observed distributions of the multijet variables are shown and compared with the theoretical predictions in Chapter 6. In Chapter 7 we show a number of studies performed to check both theoretical and experimental uncertainties. Finally, conclusions are given in Chapter 8. 

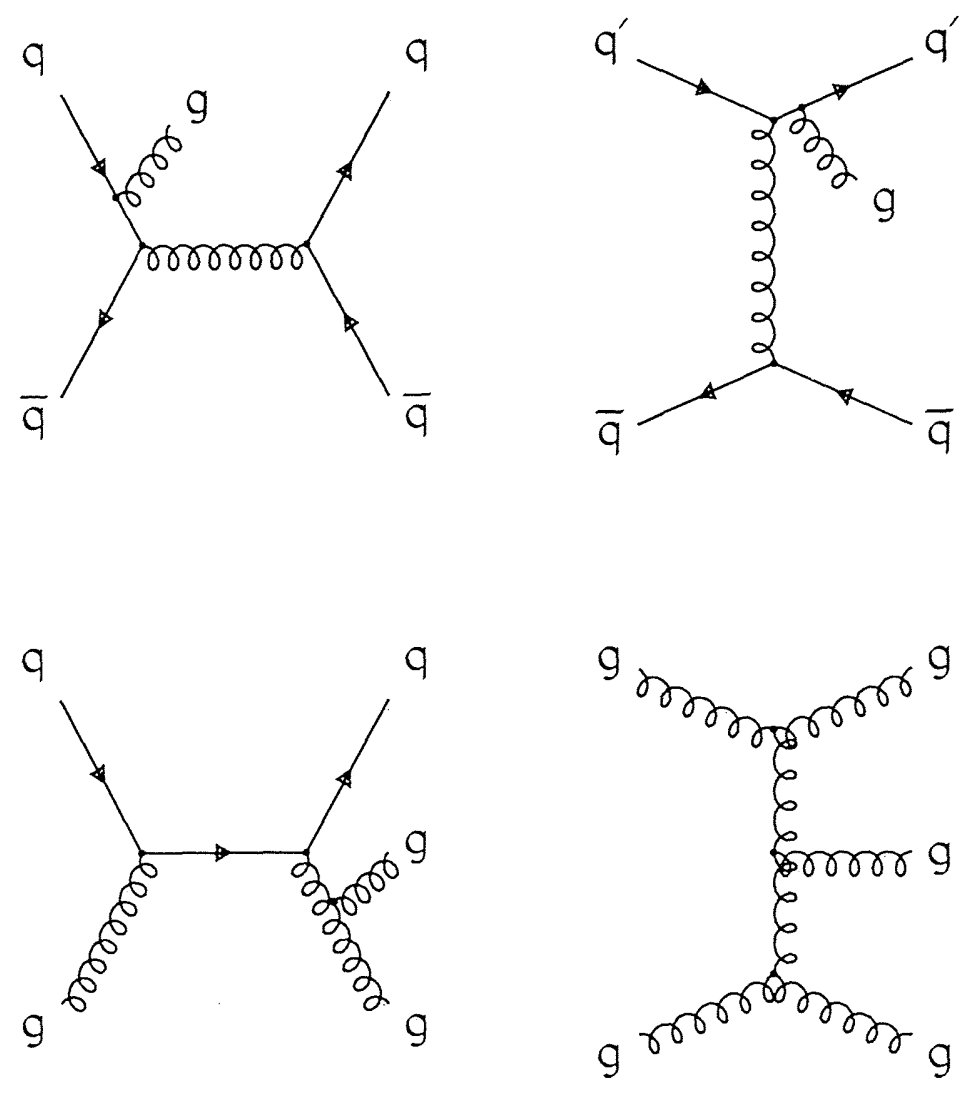

Figure 1.1: Examples of the Feynman diagrams for $2 \rightarrow 3$ subprocesses. 

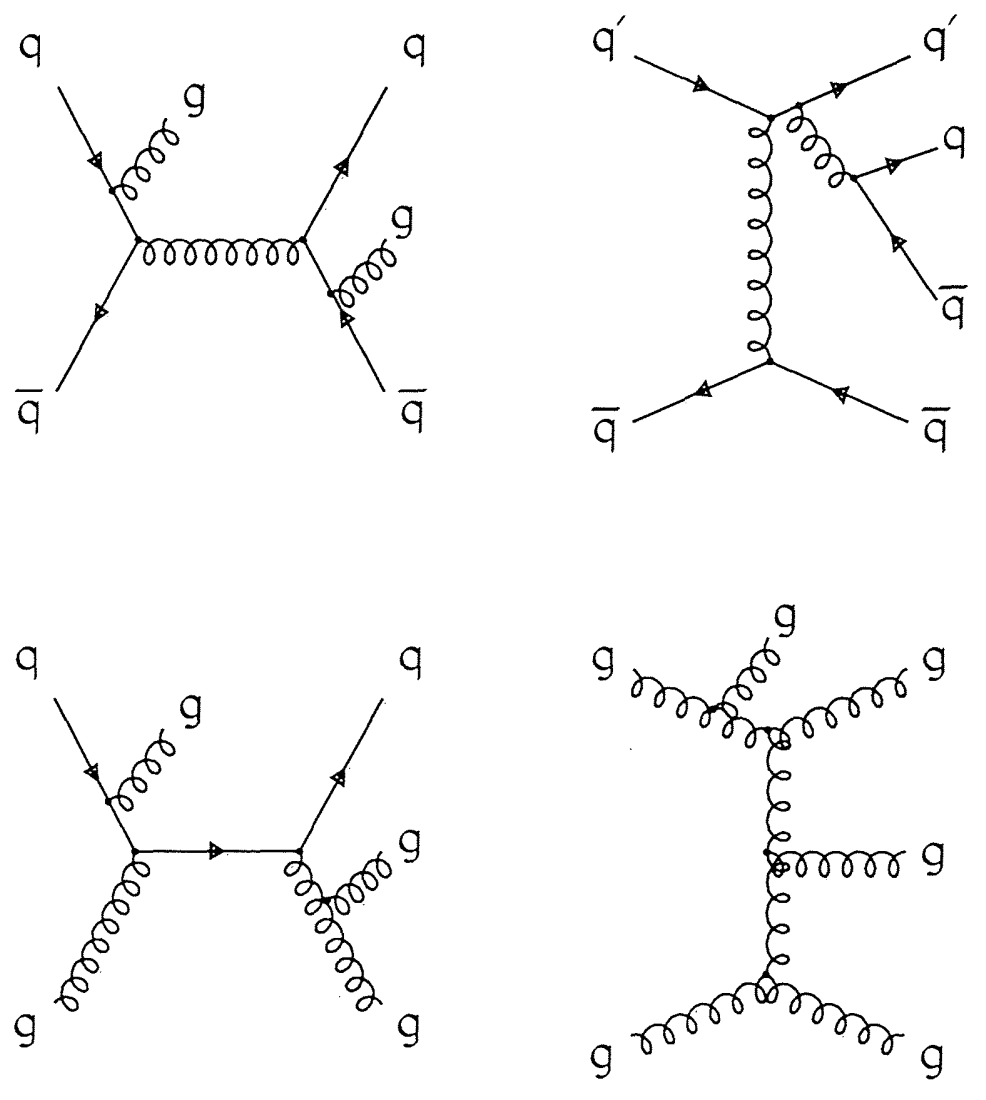

Figure 1.2: Examples of the Feynman diagrams for $2 \rightarrow 4$ subprocesses. 

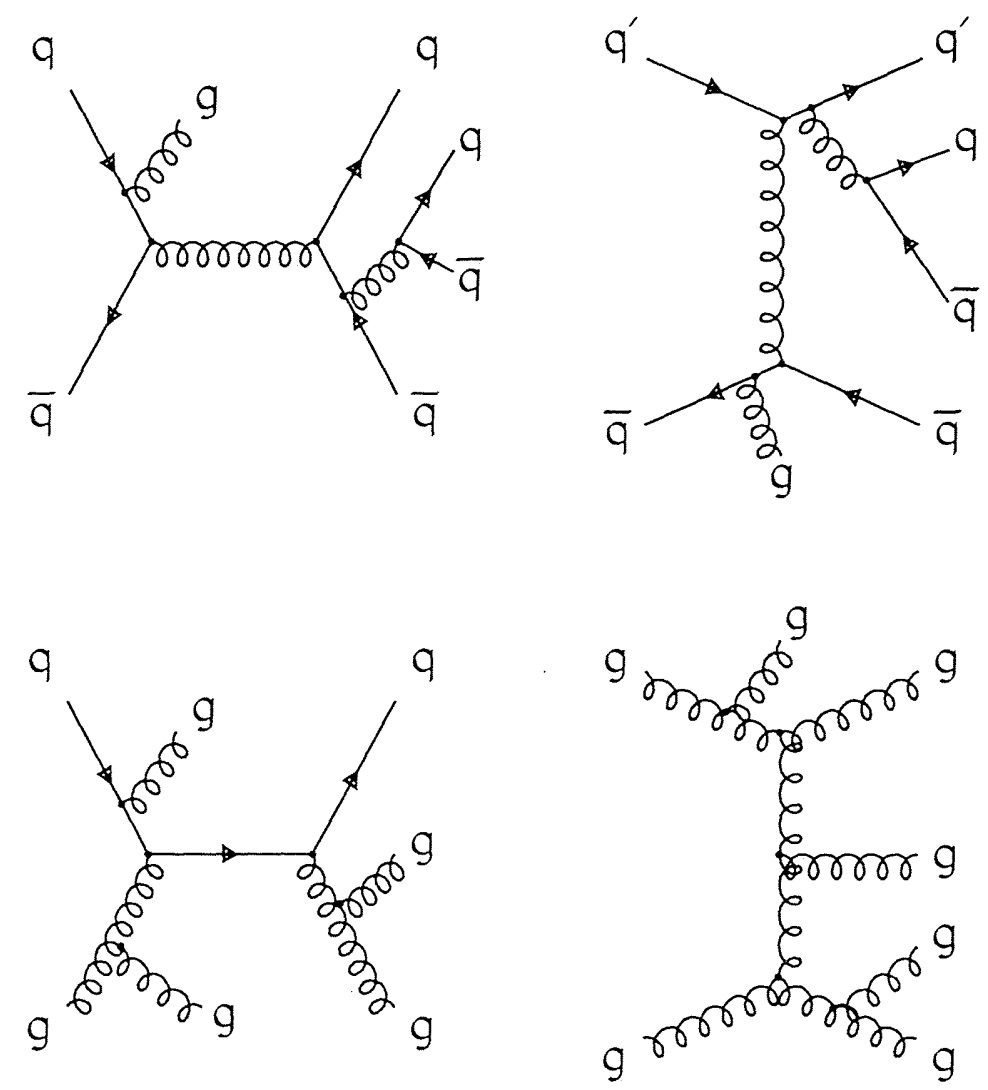

Figure 1.3: Examples of the Feynman diagrams for $2 \rightarrow 5$ subprocesses. 


\section{Chapter 2}

\section{Apparatus}

\subsection{The Fermilab Tevatron $\bar{p} p$ Collider}

The Tevatron collider has provided the highest energy protons (900 GeV) and antiprotons $(900 \mathrm{GeV})$ colliding at a center of mass energy of $1.8 \mathrm{TeV}$ since 1985 . The Tevatron collider complex consists of five stages of accelerators as illustrated in Figure 2.1. First negatively ionized hydrogen ions are injected into a DC voltage accelerator (CockcroftWalton electrostatic accelerator). They are accelerated up to $750 \mathrm{keV}$, then passed to a 500-foot-long linear accelerator (Linac). In the next stage the Linac accelerates these particles to an energy of $200 \mathrm{MeV}$. (The energy will be raised soon to $400 \mathrm{MeV}$.) The two electrons are then stripped off the hydrogen ions, leaving bare protons, which are injected to the third stage known as the Booster, a synchrotron with a diameter of approximately 500 feet. The Booster accelerates the protons to $8 \mathrm{GeV}$. The protons are then injected into the Main Ring, a synchrotron with a diameter of $2 \mathrm{~km}$, which is composed of water-cooled magnets. The protons are accelerated to $150 \mathrm{GeV}$ in the Main Ring, and finally transferred to the Tevatron Ring, a synchrotron with a diameter of two $\mathrm{km}$, composed of superconducting magnets, where they are accelerated to $900 \mathrm{GeV}$. Protons accelerated to $150 \mathrm{GeV}$ are also used to initiate production of the antiprotons. Protons provided by the Main Ring strike a tungsten target and produce antiprotons. The antiprotons are collected in the Debuncher Ring which is operated at $8 \mathrm{GeV}$. The captured beam of antiprotons, circulating the Debuncher Ring, is then made more dense by a process called stochastic cooling. The antiprotons are then transferred to the Accumulator Ring where the antiprotons are merged into a single 
beam, cooled further and stored.

The product of luminosity and cross section give the number of events produced:

$$
N=\mathcal{L} \cdot \sigma
$$

where the luminosity $\mathcal{L}$ is given by

$$
\mathcal{L}=\frac{N_{p} N_{\bar{p}} f B}{4 \pi \epsilon \beta}
$$

\begin{tabular}{lllc} 
& & typical value \\
\hline$N_{p}:$ number of protons per bunch & $1 \times 10^{11}$ \\
$N_{\bar{p}}:$ number of antiprotons per bunch & $7 \times 10^{10}$ \\
$f$ & $:$ revolution frequency $(\mathrm{kHz})$ & 50 \\
$B$ & $:$ number of bunches & 6 \\
$\epsilon$ & $:$ emittance (mm mrad) & $2.6 \times 10^{-3}$ \\
$\beta$ & $:$ betatron oscillation length $(\mathrm{m})$ & 0.5 \\
\hline
\end{tabular}

A large number of particles in a bunch or small beam size provides a large luminosity. The Tevatron provided an instantaneous luminosity of $\mathcal{L} \sim 1 \times 10^{31} \mathrm{~cm}^{-2} \mathrm{~s}^{-1}$ during the period of 1992-1995 run. An integrated luminosity of $105 \mathrm{pb}^{-1}$ has been collected by the CDF.

\subsection{The CDF Detector}

The CDF detector is a general-purpose detector designed to study the physics of $\bar{p} p$ collisions. In this section we briefly summarize the major components of the detector. A detailed description of the CDF detector can be found in [18]. The CDF has both azimuthal and forward-backward symmetry. A super conducting solenoid of length 4.8 $\mathrm{m}$ and radius $1.5 \mathrm{~m}$ generates a $1.4-\mathrm{T}$ magnetic field and contains tracking chambers used to detect charged particles, and measure the signs of their charges and their momenta. Surrounding the solenoid are calorimeters used to measure the electromagnetic and hadronic energy of jets and electrons. Outside the calorimeters are drift chambers used for muon detection. A side-view cross-section of the CDF detector is shown in 
Figure 2.2. The polar angle $(\theta)$ in spherical coordinates is measured from the proton beam axis, and the azimuthal angle $(\phi)$ from the plane of the Tevatron.

In the following subsections, we will briefly summarize the CDF detector elements. The CDF detector consists of beam-beam counters, tracking detectors, muon detectors, and calorimeters. We will begin by noting the beam-beam counter. Following that the tracking detectors and the muon detectors will be briefly described. Finally, we will describe the calorimeters which are most relevant to this analysis.

\subsubsection{Beam-Beam Counter}

There are planes of scintillation counters on the front face of each of the forward and backward shower calorimeters. These scintillators, called the beam-beam counters (BBC), provide a "minimum-bias" trigger initiating the data acquisition for the detector. The BBC counters are also used as the primary luminosity monitor.

\subsubsection{Tracking Detector}

The CDF uses three types of tracking systems which detect tracks of charged particles within the magnetic field for different purposes:

SVX: Immediately outside of the beam pipe is a system of four-layer silicon microstrip vertex chambers (SVX). The SVX is surrounding the 1.9-cm radius beryllium beampipe and consists of two identical cylindrical modules $51 \mathrm{~cm}$ long. The four layers of the SVX are at distances of 3.0, 4.2, 5.7 and $7.9 \mathrm{~cm}$ from the beamline. Using this system one can measure tracks of charged particles near the primary vertex precisely and therefore can measure impact parameters of particles with long lifetimes.

VTX: Outside of the SVX is a vertex drift chamber (VTX), which provides tracking information up to a radius of $22 \mathrm{~cm}$ in the pseudorapidity region, $|\eta|<3.25$, where $\eta \equiv-\ln (\tan (\theta / 2))$. The VTX is used to measure the $\bar{p} p$ interaction vertex along the $z$-axis with a resolution of $1 \mathrm{~mm}$.

CTC: Both the SVX and VTX are mounted inside the central tracking chamber (CTC), which is a $3.2-\mathrm{m}$-long drift chamber with an outer radius of $132 \mathrm{~cm}$ containing 
84 concentric, cylindrical layers of sense wires. Sixty layers have wires parallel to the beam direction (axial wires) and provide tracking in the $\eta-\phi$ plane. Twenty-four layers (stereo wires) are titled at +3 degrees or -3 degrees with respect to the beam direction. Together, the axial and stereo wires provide tracking in the $r-z$ plane. The CTC provides a precision momentum determination of charged particles. The momentum resolution of the SVX-CTC system is $\sigma_{p_{T}} / p_{T}=\left[\left(0.0009 p_{T}\right)^{2}+(0.0066)^{2}\right]^{1 / 2}$, where $p_{T}$ has units of $\mathrm{GeV} / c$, with a resolution of $\sigma_{p_{T}} / p_{T}^{2}<0.002(\mathrm{GeV} / c)^{-1}$ for isolated charged particles.

\subsubsection{Muon Detector}

Sets of muon detection systems are located outside of the calorimeters:

CMU: The central muon detection system consists of the four layers of drift chambers located outside the central hadronic calorimeter. This system covers approximately 84 $\%$ of the solid angle for $|\eta|<0.6$ and can be reached by muons with $p_{T}$ in excess of 1.4 $\mathrm{GeV} / c$.

CMP: This system consists of four layers of drift chambers located behind the CMU and is referred to as the central muon upgrade. This system covers $63 \%$ of the solid angle for $|\eta|<0.6$.

CMX: This system is called the central muon extension and consists of drift chambers and scintillation counters. This system covers approximately $71 \%$ of the solid angle for $0.6<|\eta|<1.0$.

\subsubsection{Calorimetry}

The solenoid and tracking volume of CDF is surrounded by calorimeters, which measure the energy and position of leptons and jets. They cover $2 \pi$ in azimuthal angle, and in pseudorapidity $\eta$ from -4.2 to 4.2 . In our multijet analysis we have used the energies and momenta of outgoing jets measured by the calorimeters. There are three separate regions of calorimeters, the so-called "central", "plug", and "forward" calorimeters. Each calorimeter consists of both an electromagnetic calorimeter and 
behind it a hadronic calorimeter. They are segmented into "towers" of 0.1 units of pseudorapidity as projected from the detector center. The towers are segmented azimuthally by $15^{\circ}$ in the central region and by $5^{\circ}$ in the plug and forward regions as shown in Figure 2.3.

CEM: The central electromagnetic calorimeter. This system uses a hybrid design with scintillator and wavelength shifter sandwiched with lead absorber plates for energy measurement and an embedded proportional strip chamber (CES) for position determination and lateral shower shape. The CEM covers a polar angle region of $39^{\circ}<\theta<141^{\circ}(|\eta|<1.1)$, and is azimuthally segmented into $15^{\circ}$ wedges mounted surrounding the solenoid. It consists of 21-31 layers of $5 \mathrm{~mm}$ polystyrene scintillator interleaved with 20-30 layers of 1/8-inch lead sheet. The CEM has a total of 965 photomultiplier tubes (PMT). The energy resolution for incident electrons and photons is given by

$$
\left(\frac{\sigma}{E}\right)^{2}=\left(\frac{13.7 \%}{\sqrt{E \sin \theta}}\right)^{2}+(2 \%)^{2}
$$

where $E$ is in $\mathrm{GeV}$ and the $\sin \theta$ factor reflects increased sampling thickness seen by electrons entering the calorimeter at an angle.

The CES contains 128 strips aligned in $\phi$ and 64 wires along the $z$-axis. It determines the electron hit position within $\pm 2 \mathrm{~mm}$ for $50 \mathrm{GeV} / c$ electrons.

CHA/WHA: The central and wall hadronic calorimeters. Both these systems overlap the CEM calorimeter, and use scintillator with wavelength shifter sandwiched with steel absorber plates for energy measurement. The CHA consists of 43 layers of $10 \mathrm{~mm}$ plastic scintillator interleaved with 32 layers of $25 \mathrm{~mm}$ iron. The CHA uses a total of 768 PMT's. The WHA consists of 15 layers of $10 \mathrm{~mm}$ plastic scintillator interleaved with 15 layers of $50 \mathrm{~mm}$ iron. The energy resolutions for incident isolated pions are approximately given by

$$
\left(\frac{\sigma}{E}\right)^{2}=\left(\frac{50 \%}{\sqrt{E \sin \theta}}\right)^{2}+(3 \%)^{2}
$$

for the CHA, and

$$
\left(\frac{\sigma}{E}\right)^{2}=\left(\frac{75 \%}{\sqrt{E}}\right)^{2}+(4 \%)^{2}
$$


for the WHA.

PEM: The plug electromagnetic calorimeter. This system uses conductive plastic proportional tube arrays sandwiched with lead absorber panels, and covers polar angle regions $10^{\circ}<\theta<36^{\circ}$ and $144^{\circ}<\theta<170^{\circ}(1.1<|\eta|<2.4)$. The PEM consists of 34 layers of gas proportional tubes, alternated with 34 layers of $2.69 \mathrm{~mm}$ thick lead absorber. The PEM has a total of 5760 channels. The energy resolution is given by

$$
\left(\frac{\sigma}{E}\right)^{2}=\left(\frac{22 \%}{\sqrt{E}}\right)^{2}+(2 \%)^{2} .
$$

PHA: The plug hadronic calorimeter. The PHA overlaps the PEM calorimeters and consists of 20 layers of gas proportional tubes interleaved with 20 layers of $50.0 \mathrm{~mm}$ thick steel absorber. The PHA has a total of 1728 channels. The energy resolution is given by

$$
\left(\frac{\sigma}{E}\right)^{2}=\left(\frac{106 \%}{\sqrt{E}}\right)^{2}+(6 \%)^{2} .
$$

FEM: The forward electromagnetic calorimeter. The FEM calorimeters are for the small angle regions and are located approximately $6.5 \mathrm{~m}$ from the interaction point in both the proton and the antiproton beam directions. The FEM covers polar angle regions $2^{\circ}<\theta<13^{\circ}$ and $167^{\circ}<\theta<178^{\circ}(2.2<|\eta|<4.2)$. The FEM calorimeter module is segmented into projective towers, whose size is $5^{\circ}$ in $\phi$ and 0.1 units in $\eta$. The FEM module has 30 sampling layers interleaved with layers of lead absorber, and a total of 5760 channels. The energy resolution is given by

$$
\left(\frac{\sigma}{E}\right)^{2}=\left(\frac{26 \%}{\sqrt{E}}\right)^{2}+(2 \%)^{2} .
$$

FHA: The forward hadronic calorimeter. The FHA overlaps the FEM calorimeters and consists of 27 sampling layers of $51 \mathrm{~mm}$ iron plate alternated with 27 lead layers. The FHA has a total of 2880 channels. The energy resolution is given by

$$
\left(\frac{\sigma}{E}\right)^{2}=\left(\frac{137 \%}{\sqrt{E}}\right)^{2}+(3 \%)^{2} .
$$

In all cases, the absorber in the hadronic calorimeter is iron, that in the electromagnetic calorimeter is lead. The $\eta$ coverages, energy resolutions, and thickness of absorbers in the calorimeter components are summarized in Table 2.1. 


\begin{tabular}{l|c|c|c}
\hline \hline System & $\eta$ range & Energy resolution & Thickness \\
\hline CEM & $|\eta|<1.1$ & $13.7 \% \sqrt{E_{T}} \oplus 2 \%$ & $18 X_{0}$ \\
PEM & $1.1<|\eta|<2.4$ & $22 \% \sqrt{E} \oplus 2 \%$ & $18-21 X_{0}$ \\
FEM & $2.2<|\eta|<4.2$ & $26 \% \sqrt{E} \oplus 2 \%$ & $25 X_{0}$ \\
CHA & $|\eta|<0.9$ & $50 \% \sqrt{E_{T}} \oplus 3 \%$ & $4.5 \lambda_{0}$ \\
WHA & $0.7<|\eta|<1.3$ & $75 \% \sqrt{E} \oplus 4 \%$ & $4.5 \lambda_{0}$ \\
PHA & $1.3<|\eta|<2.4$ & $106 \% \sqrt{E} \oplus 6 \%$ & $5.7 \lambda_{0}$ \\
FHA & $2.4<|\eta|<4.2$ & $137 \% \sqrt{E} \oplus 3 \%$ & $7.7 \lambda_{0}$ \\
\hline \hline
\end{tabular}

Table 2.1: Summary of CDF calorimeter properties. The symbol $\oplus$ signifies that the constant term is added in quadrature in the resolution. Energy resolutions for the electromagnetic calorimeters are for incident electrons and photons, and for hadronic calorimeters are for incident isolated pions. Energy given in $\mathrm{GeV}$. Thicknesses are given in radiation lengths $\left(X_{0}\right)$ and interaction lengths $(\lambda)$ for the electromagnetic and hadronic calorimeters, respectively. 


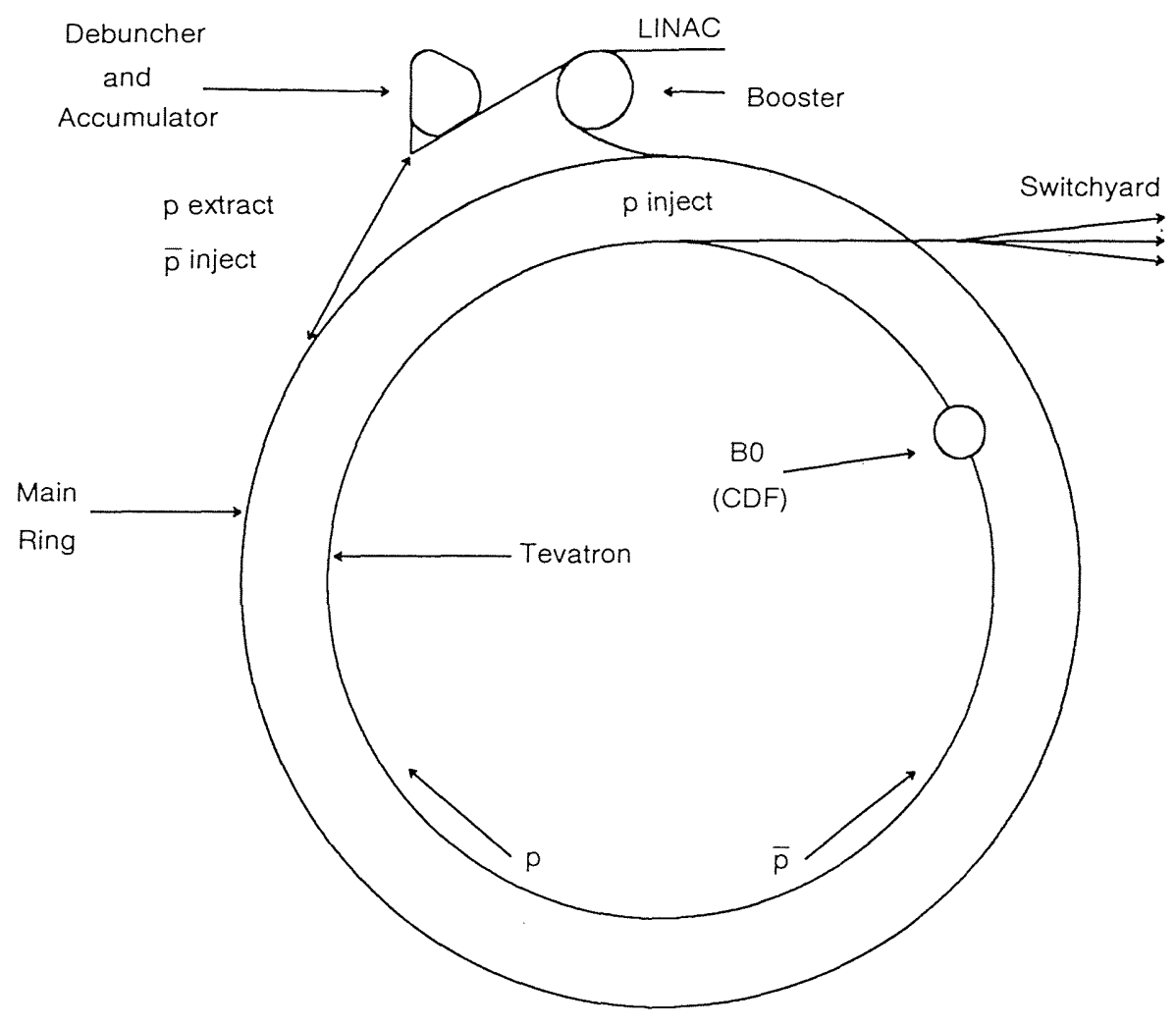

Figure 2.1: The perspective view of the Tevatron Proton-Antiproton Collider at Fermi National Accelerator Laboratory. 


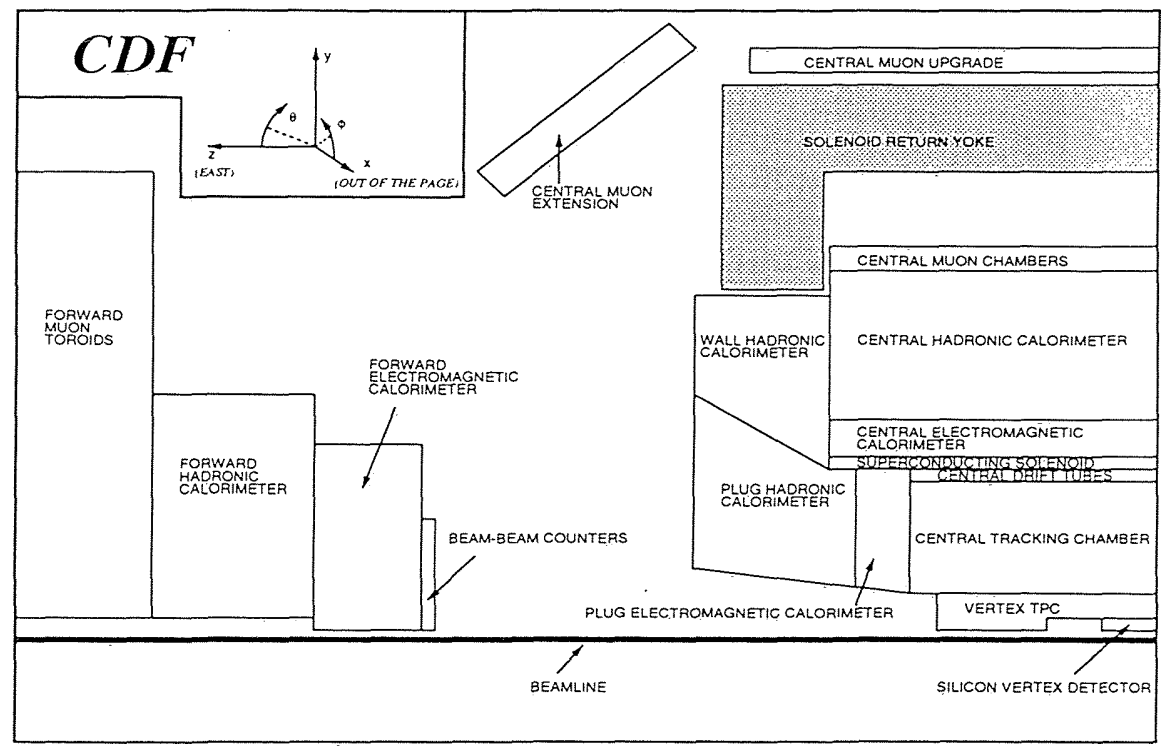

Figure 2.2: Perspective view of the Collider Detector at Fermilab. 


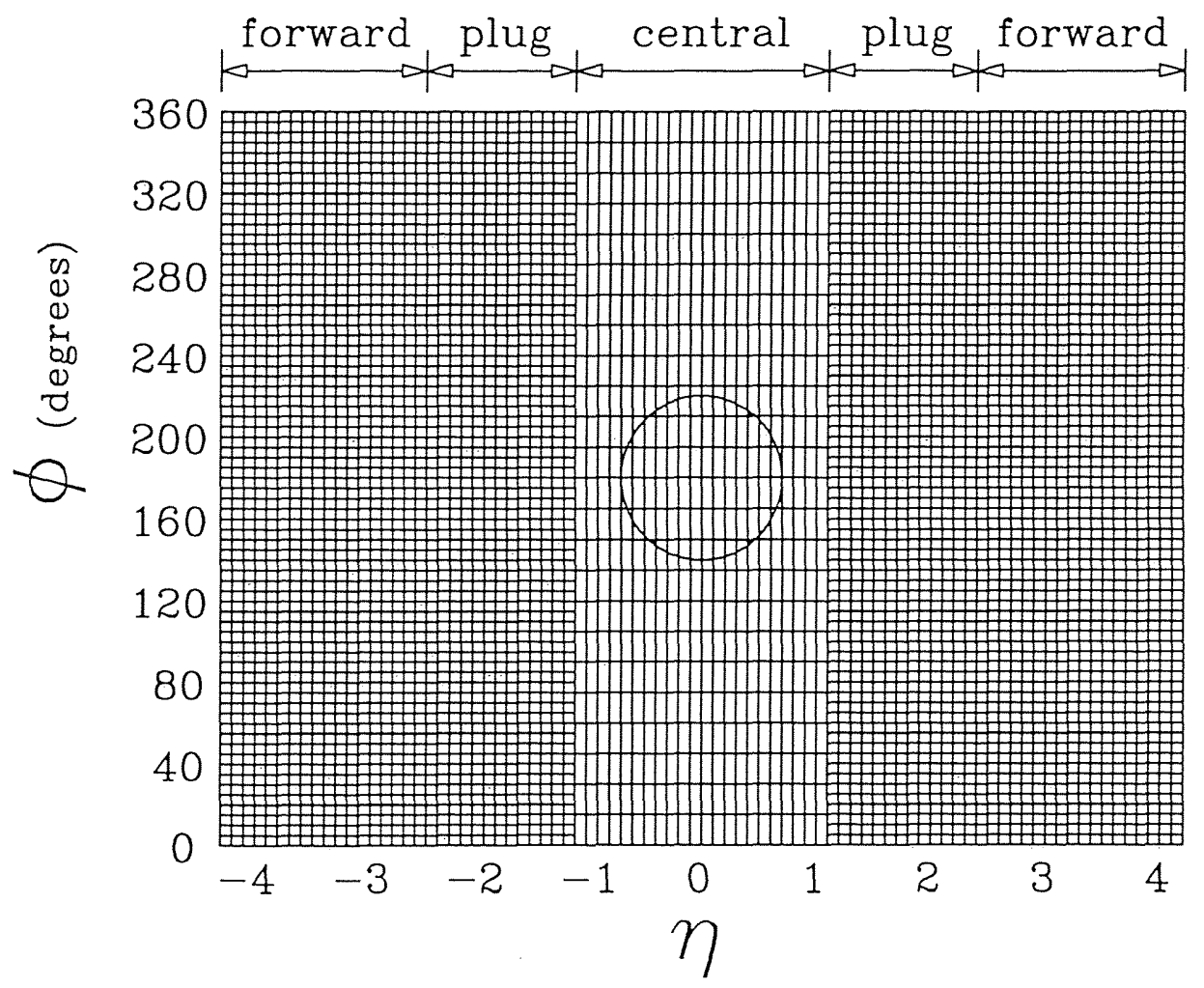

Figure 2.3: The $\eta-\phi$ segmentation of the CDF calorimeter. Also shown is the size of a cone cluster with a 0.7 radius. This is the cone clustering radius used in our analysis. 


\section{Chapter 3}

\section{Data Set}

The data which has been used in our analysis were recorded by the CDF collaboration during the period 1992-1995, and corresponds to an integrated luminosity of $105 \mathrm{pb}^{-1}$. In this chapter we will describe the CDF data acquisition and triggers (Section 3.1), and the selection of events with large total transverse energies (Section 3.2).

\subsection{Data Acquisition}

\subsubsection{CDF Data Flow}

The CDF uses 4 levels of trigger stages, numbered 0 to 3 . The first stage, Level 0 , requires a coincidence between hits of the east and west beam-beam counters. The Level 0 trigger outputs signals at a rate of $\sim 50 \mathrm{kHz}$. The Level 1-3 triggers consist of a logical "OR" of several requirements which are defined to detect electrons, photons, muons, missing energy, tau leptons and jets, and select events with physics interest. The data used in our analysis were collected with a $\sum E_{T}$ trigger. This trigger asks an event to have a very large total transverse energy, where the sum is over all outgoing jets. We begin by noting the CDF jet clustering algorithm. Then the $\sum E_{T}$ triggers at Level 2 and 3 will be described.

\subsubsection{Definition of Jet}

The CDF jet clustering algorithm uses a cone of a fixed radius to define a jet [14]. This definition is similar to that used by the UA1 experiment [19]. The jet-finding 
algorithm begins by creating a list of towers above a fixed $E_{T}$ threshold (=1.0 GeV) to be used as "seeds" for the jet finder. In the plug and forward calorimeter regions, towers are grouped together in sets of three in $\phi$, spanning 15 degrees to correspond to the central segmentation. Preclusters are formed from an unbroken chain of contiguous seed towers with a continuously decreasing tower $E_{T}$. If a tower is outside a window of $7 \times 7$ towers surrounding the seed, it is used to form a new precluster. These preclusters are used as a starting point for cone clustering. The preclusters then are grown into clusters using the true tower segmentation (i.e. no ganging). First, the $E_{T}$ weighted centroid of the precluster is found and a cone of radius $R_{0}$ in $\eta$ - $\phi$ space is formed around the centroid. In our analysis the cone radius $R_{0}=0.7$ was chosen (see Figure 2.3). The choice of $R_{0}=0.7$ is based partly on the distribution of energy flow with respect to the jet axis in events dominated by two jets. The CDF group studied and found that cone sizes as small as 0.4 , or as large as 1.0 may be sensible to the azimuthal energy flow with the jet axis in two-jet events [14]. Other studies, for example UA2 [20], give evidence that a range of $0.4<R_{0}<1.0$ yield good resolution.

Then, all towers with an $E_{T}$ of at least $100 \mathrm{MeV}$ are incorporated into the cluster. A tower is included in a cluster if its centroid is inside the cone; otherwise it is excluded. A new cluster center is calculated from the set of towers within the clustering cone, again using an $E_{T}$ weighted centroid, and a new cone is drawn about this position. The process of recomputing a centroid and finding new or deleting old towers is iterated until the tower list remains unchanged. For our multijet studies, it is important to handle properly conditions where two clusters overlap, particularly for final-state gluon emission where the gluon can merge into the jets. There are four possible overlap conditions: If two clusters are distinct, they are left alone. If one cluster is completely contained in another, the smaller of the two is dropped. If the towers have some finite overlap, then an overlap fraction is computed as the sum of the $E_{T}$ of the common towers divided by the $E_{T}$ of the smaller cluster. If the fraction is above a cutoff $(0.75)$ then the two clusters are combined. If the fraction is less than the cut, the clusters are kept intact. In this case, each tower in the overlap region is assigned to the cluster closest in $\eta$ - $\phi$ space. After the clusters are uniquely assigned to towers, the centroids are recomputed. As with the original cluster finding, the process of centroid computation 
and tower reshuffling is iterative, and ends when the tower lists remain fixed.

The separation between jets $\Delta R$ is usually defined in $\eta-\phi$ space as

$$
\Delta R \equiv \sqrt{(\Delta \eta)^{2}+(\Delta \phi)^{2}},
$$

where $\Delta \eta$ and $\Delta \phi$ are distances of two jets in $\eta$ and $\phi$, respectively. Figure 3.1 shows the observed $\Delta R$ distribution of inclusive five-jet events with the clustering cone radius $R_{0}=0.7$. As shown in the figure, a plot of the separation between all jet-jet pairs observed in the data sample reveals that to a good approximation clusters with separations $\Delta R<0.8$ are always merged by the jet algorithm into a single jet, and clusters with separations $\Delta R>1.0$ are never merged $[1,14]$. Thus, the effective minimum observable separation between jets is

$$
\Delta R_{\min }=0.9 \pm 0.1
$$

From the towers associated with the cluster, the quantities $\left(p_{x}, p_{y}, p_{z}, E\right)$ are calculated. The electromagnetic and hadronic compartments of each tower are assigned massless four-vectors with magnitude equal to the energy deposited in the tower and with the direction defined by a unit vector pointing from the event origin to the center of the face of the calorimeter tower (calculated at the depth that corresponds to shower maximum). $E$ is the scalar sum of tower energies, $p_{x}$ is the sum of $p_{x_{i}}$ where $i$ is the tower index. Other quantities, for example the jet transverse energy $E_{T}$ and the jet momentum $\vec{P}$, can then be determined:

$$
\begin{aligned}
& E_{T} \equiv E \sin \theta=E \frac{\sqrt{p_{x}^{2}+p_{y}^{2}}}{\sqrt{p_{x}^{2}+p_{y}^{2}+p_{z}^{2}}} \text { and } \\
& \vec{P} \equiv \sum_{i} \overrightarrow{n_{i}} E_{i},
\end{aligned}
$$

where $\theta$ is the angle between the beam axis and the jet direction, $\overrightarrow{n_{i}}$ is the unit vector pointing from the event origin to the $i$-th tower, and $E_{i}$ is the energy deposited in the $i$-th tower.

\subsection{3 $\sum E_{T}$ trigger}

At Level 2, the trigger hardware performs clustering in $\eta$ - $\phi$ space of the energy depositions in the calorimeters. A cluster is initiated by a seed tower with $E_{T}$ above 
$3 \mathrm{GeV}$, and consists of all contiguous towers in $\eta$ and $\phi$ with $E_{T}$ above $1 \mathrm{GeV}$. The $\sum E_{T}$ trigger is formed by summing over these clusters and asking

$$
\sum E_{T}>175 \mathrm{GeV}
$$

where the sum is over all calorimeter clusters with $E_{T}>10 \mathrm{GeV}$ in the central, plug and forward calorimeters.

At Level 3, a subset of the events that passed the Level 2 trigger is selected by an online computer farm by requiring

$$
\sum E_{T}>300 \mathrm{GeV}
$$

where the sum is over all calorimeter clusters with $E_{T}>10 \mathrm{GeV}$ reconstructed with the CDF jet clustering algorithm described above. Transverse energies were computed assuming that the event-vertex was at $z=0$. The high- $\sum E_{T}$ in many of these events can be attributed to a high energy cosmic-ray interaction, a beam halo, or a detector malfunction. They are expected to exhibit a large transverse-energy imbalance. To suppress these events, we have applied the following requirement:

The $E_{T}$ significance

$$
S \equiv \frac{E_{T}}{\sqrt{\sum E_{T}}}<10
$$

where the missing transverse energy $E_{T}$ is given by

$$
E_{T} \equiv\left|\sum_{i} \vec{E}_{T_{i}}\right|
$$

and $\vec{E}_{T_{i}}$ is the vector which points from the interaction point (at $z=0$ ) to the calorimeter tower with magnitude equal to $E_{T_{i}}$.

The Figure 3.2 shows the dependence of the $\sum E_{T}$ Level 2 and Level 3 trigger cross-sections on the run number. In the figure we find that both trigger cross-sections are stable with run numbers in which events were detected at various instantaneous luminosities.

\section{2 $\sum E_{T}$ Data Sample}

This section describes jet energy corrections and the further selection of high- $\sum E_{T}$ events. 


\subsubsection{Jet Energy Corrections}

The transverse energies and momenta of jets defined with the CDF jet clustering algorithm depend only on the energy deposition observed in the calorimeters. These quantities differ from the true values because:

(i) The calorimeter response for low-energy charged pions with momenta below 10 $\mathrm{GeV} / c$ is non-linear.

(ii) Charged particles with transverse momenta below $400 \mathrm{MeV} / \mathrm{c}$ bend in the magnetic field and do not reach the calorimeter.

(iii) Particles that shower in boundary regions of the calorimeter, on average, will have a smaller energy reported than for regions of uniform response.

(iv) Energy not associated with the hard-scattering process will be collected within the clustering cone (so-called "underlying event").

(v) Transverse spreading of the jet due to fragmentation effects will cause particles to be lost outside the clustering cone.

A correction function which takes into account these effects is generated and applied to jets in the data sample [14]. The jet corrections are larger for lower $E_{T}$ jets, and typically increase jet energies by $30 \%$ (40\%) for jets with $E_{T}=40 \mathrm{GeV}(20 \mathrm{GeV})$. After the correction, jet energies are measured with a precision $\sigma_{E} / E$ of approximately 0.1 and multijet masses calculated from the jet four-vectors are measured with a precision $\sigma_{m} / m$ of approximately 0.1 . The systematic uncertainty on the jet energy scale is approximately $7 \%[14]$.

\subsubsection{Event Selection}

The initial sample which passed the trigger requirements described in the previous section still contains some cosmic-ray interactions and events with noise in the calorimeters. The cosmic-rays or detector noise provide events with large total transverse energies independent of the proton-antiproton collisions. Such events are expected 
sometime to have a large energy deposit which exceeds the total energy of the incoming particles $(1.8 \mathrm{TeV})$, to have no reconstructed primary vertex near the center $(z=0)$ of the detector, and/or to have a large transverse-energy imbalance. To further reject these backgrounds, events are rejected if:

(i) There is significant energy deposited in the hadron calorimeter out-of-time with the proton-antiproton collision.

(ii) The total energy deposited in the calorimeters exceeds $2000 \mathrm{GeV}$.

(iii) There is no reconstructed primary vertex with $|z|<60 \mathrm{~cm}$.

(iv) $S>6$.

Figure 3.3-(a) shows the observed $E_{T}$ significance distribution. It rapidly decreases as $S$ increases and has a long tail associated with cosmic-rays or detector noise. Figure 3.3(b) shows the observed the $E_{T}$ distribution for $S<6$ compared with the predicted distributions by the HERWIG + the CDF detector simulation (described in Chapter 4). The observed $E_{T}$ distribution after applying the cut of $S<6$ is seen to be in fair agreement with the HERWIG prediction. The predicted distribution in the figures can be thought of as reflects the experimental resolution on the measurement of the $E_{T}$.

We wish to select events based on their total transverse energies after the reconstructed jets have been corrected for calorimeter non-linearities, energy lost in uninstrumented regions and outside of the clustering cone, and energy gained from the underlying event. The $\sum E_{T}$ was recalculated using the reconstructed vertex position and corrected jet energies, and summing over all jets with $E_{T}>20 \mathrm{GeV}$. The resulting $\sum E_{T}$ distribution peaks around $400 \mathrm{GeV}$ as shown in Figure 3.4-(a). At lower $\sum E_{T}$ the $\sum E_{T}$ trigger requirements are no longer fully efficient. This is because in the online computer farm we calculate the $\sum E_{T}$ using the center of the detector as the vertex position whereas offline we use the reconstructed event vertex. Figure 3.5 shows that reconstructed vertex distribution. It has a peak at $z=0$ and has long tails up to $|z|=100 \mathrm{~cm}$. In Figure 3.6 shown the scatter plots of the corrected and the uncorrected $\sum E_{T}$. Typically the jet corrections increase $\sum E_{T}$ by $25 \%$. We then 
apply a cut of

$$
\sum E_{T}>420 \mathrm{GeV}
$$

Figure 3.4-(b) shows the observed $\sum E_{T}$ distribution compared with the HERWIG prediction for $\sum E_{T}>420 \mathrm{GeV}$. The parton shower Mote Carlo prediction gives a fair description of the observed distribution, although there is some indication that it underestimates the observed rate at high $\sum E_{T}$. More details of the CDF high- $\sum E_{T}$ data sample are described in Ref. [21]. Finally, a total of 30707 events survived the selection described above.

\subsubsection{Further Selection of Multijet Events}

In our analysis all events are inclusive. If there are more than $\mathrm{N}$ jets in an event, the $\mathrm{N}$ highest- $E_{T}$ jets are used to define the $\mathrm{N}$-jet system for $\mathrm{N}=3,4$, and 5 . It should be noted that at fixed multijet mass the following requirements place restrictions on the available multijet parameter space:

$$
\sum E_{T}>420 \mathrm{GeV}
$$

where the sum is over the leading $\mathrm{N}$ jets with $E_{T}>20 \mathrm{GeV}$, AND

$$
\Delta R_{\min }>0.9
$$

where $\Delta R_{\min }$ is the minimum $\Delta R$ between two jets among the leading $\mathrm{N}$ jets. The numbers of three-, four-, and five-jet inclusive events after applying the above selection cuts are summarized in Table 3.1 (first column). 


\begin{tabular}{c|c|c}
\hline \multirow{2}{*}{$\begin{array}{c}\text { Data } \\
\text { Sample }\end{array}$} & $\begin{array}{c}\mid c \\
\text { number of events } \\
\text { selection }\end{array}$ & $\begin{array}{l}\text { after the cuts on } \\
\text { multijet variables }\end{array}$ \\
\hline 3-jet Inclusive & 21830 & 1053 \\
4-jet Inclusive & 10928 & 1298 \\
5-jet Inclusive & 4009 & 804 \\
\hline \hline
\end{tabular}

Table 3.1: Numbers of events remaining after the multijet event selection (1st column) and after applying the cuts on multijet variables (2nd column). 


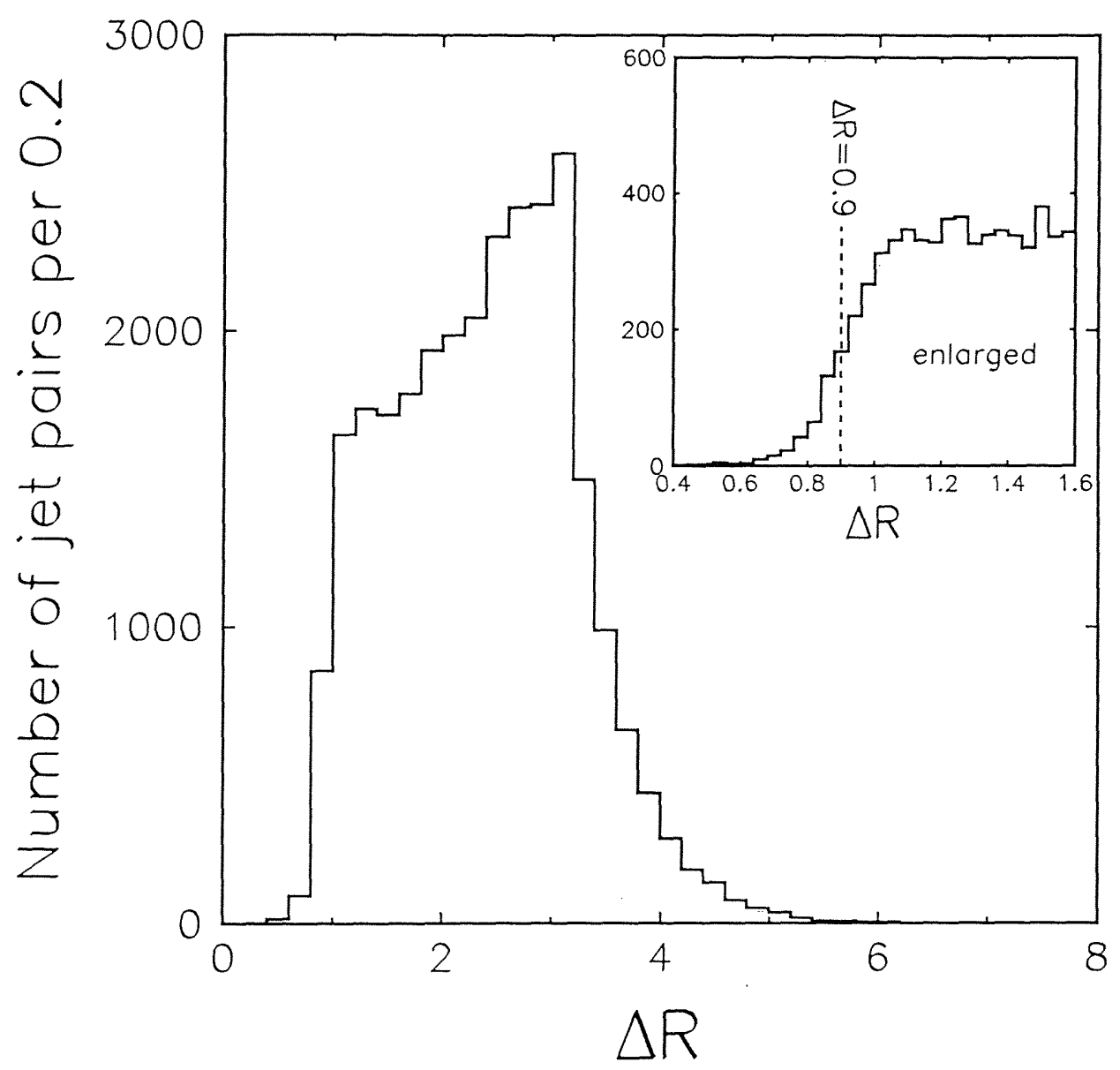

Figure 3.1: The observed distribution of $\Delta R$ between all jet-jet pairs in inclusive five-jet events. 
( $\left.\Sigma E_{\mathrm{T}}\right)$ Cross-section vs. Run Number
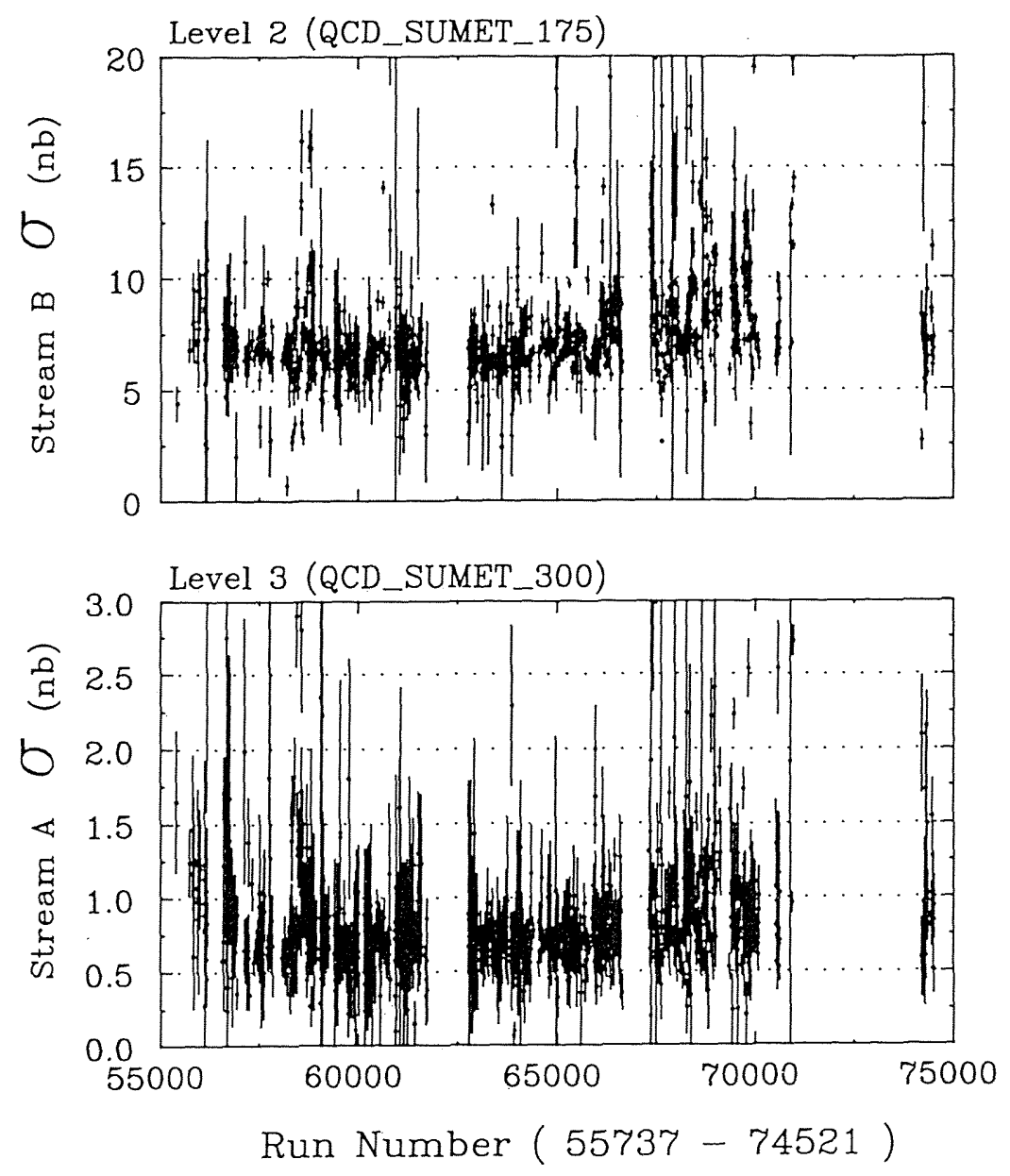

Figure 3.2: The cross-section distributions of the Level 2 and Level $3 \sum E_{T}$ triggers as a fuction of run number. 

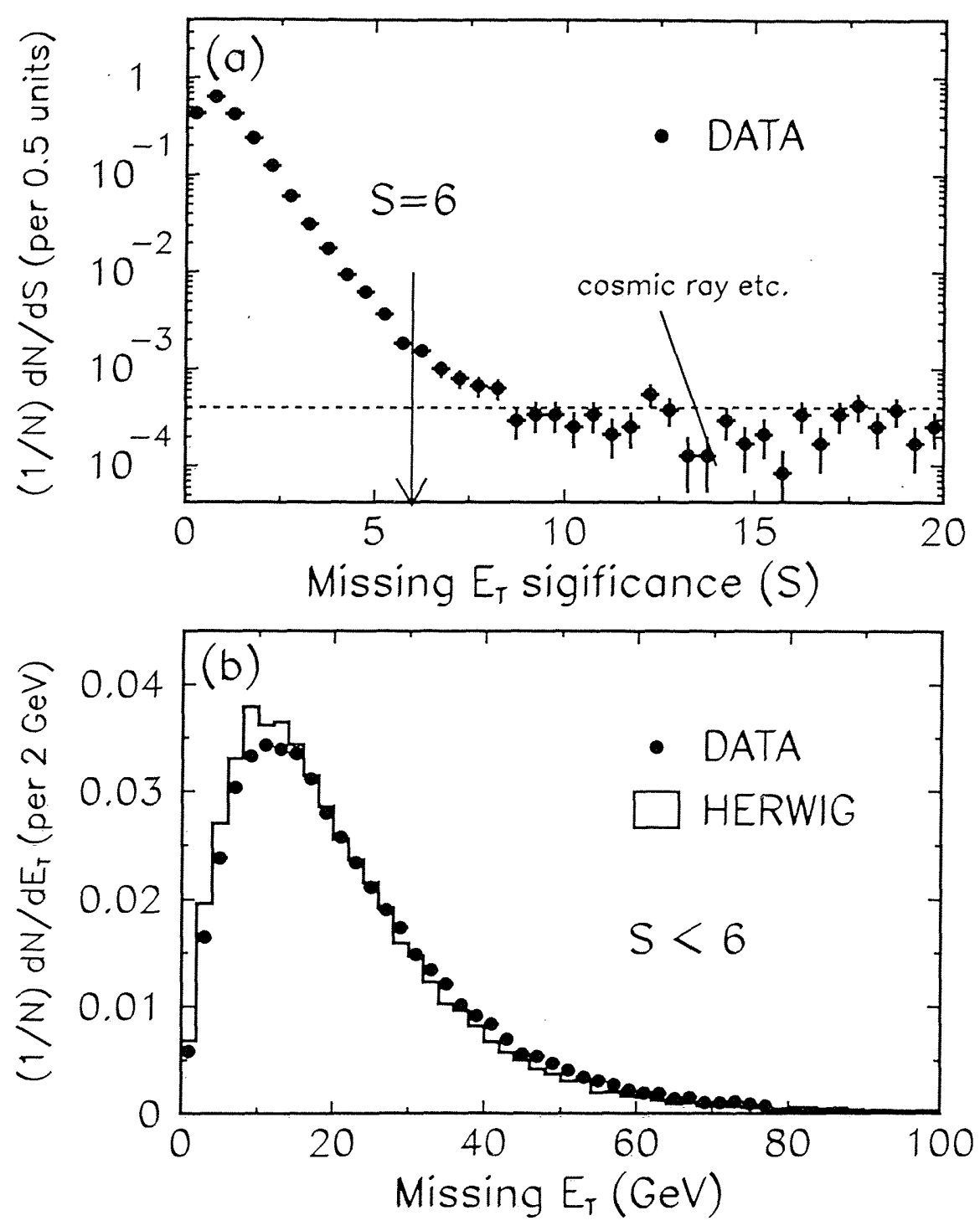

Figure 3.3: The observed missing $E_{T}$ distributions: (a) the observed missing $E_{T}$ significance distribution (points) and (b) the observed missing $E_{T}$ distribution (points) compared with the HERWIG predictions (histogram). 

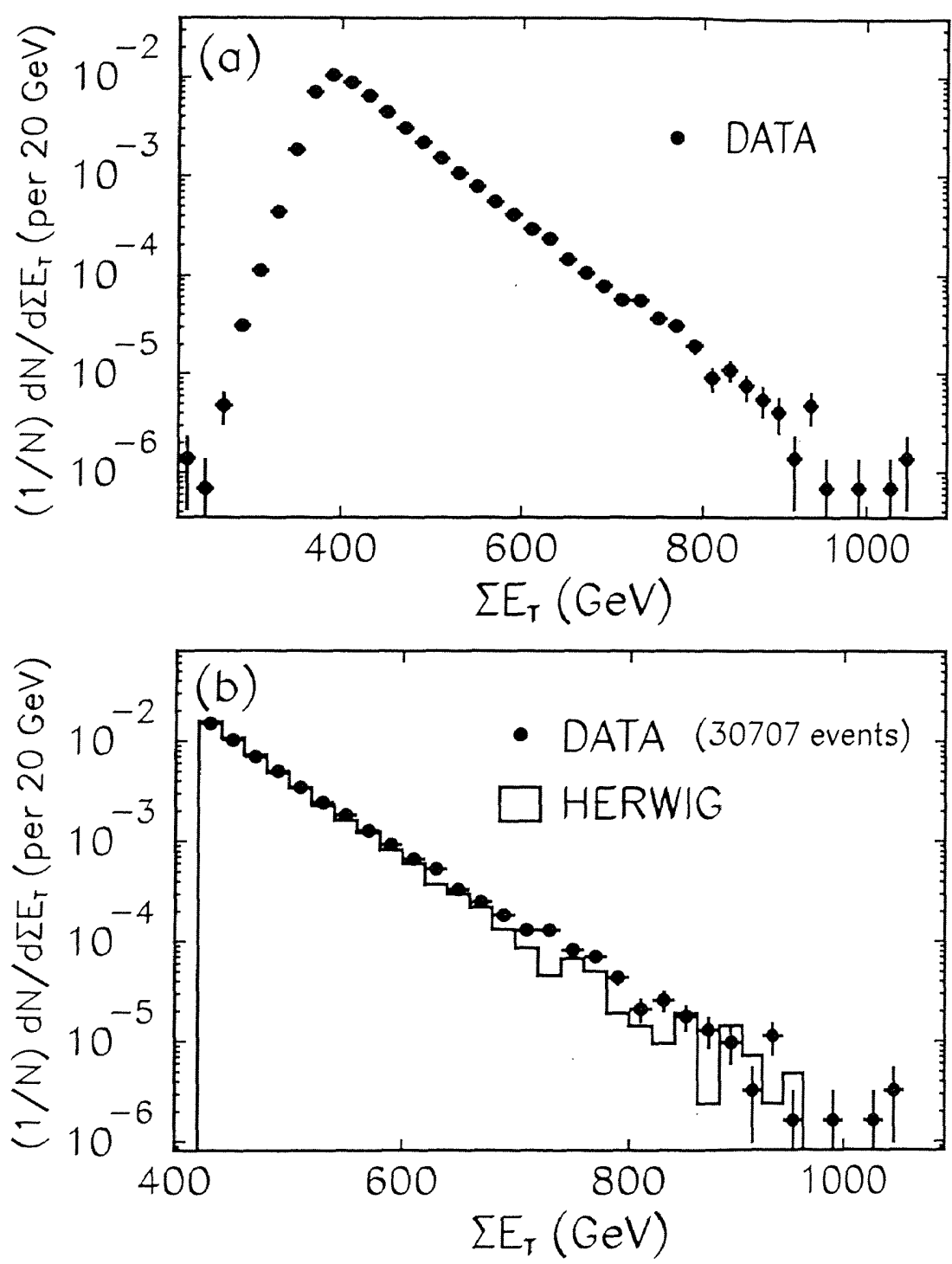

Figure 3.4: The observed $\sum E_{T}$ distributions: (a) the observed distribution (points) and (b) the observed distribution (points) compared with the HERWIG prediction (histograms) after applying the requirement of $\sum E_{T}>420 \mathrm{GeV}$. 


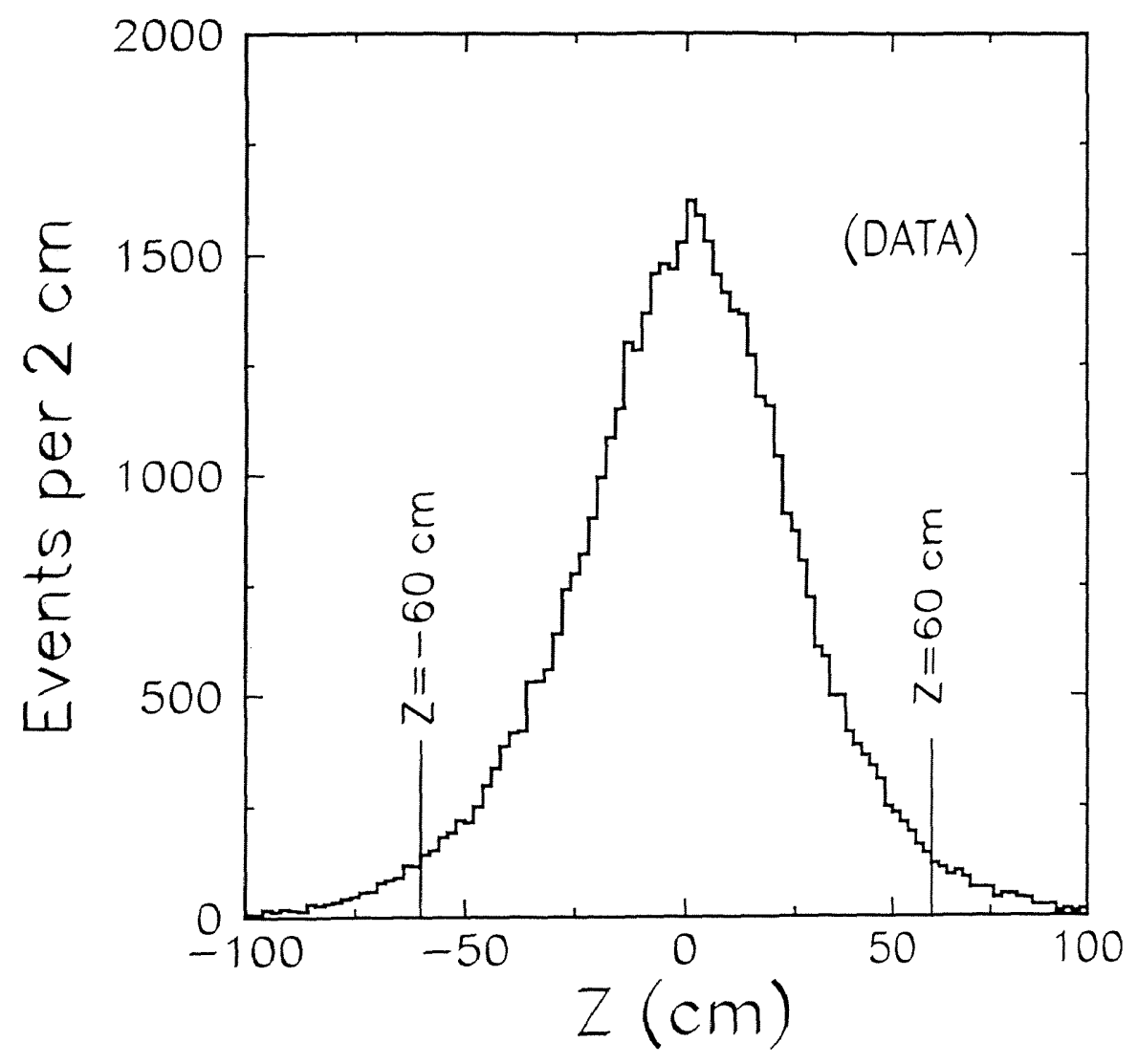

Figure 3.5: The reconstructed vertex ( $z$ position) distribution of the observed events. 


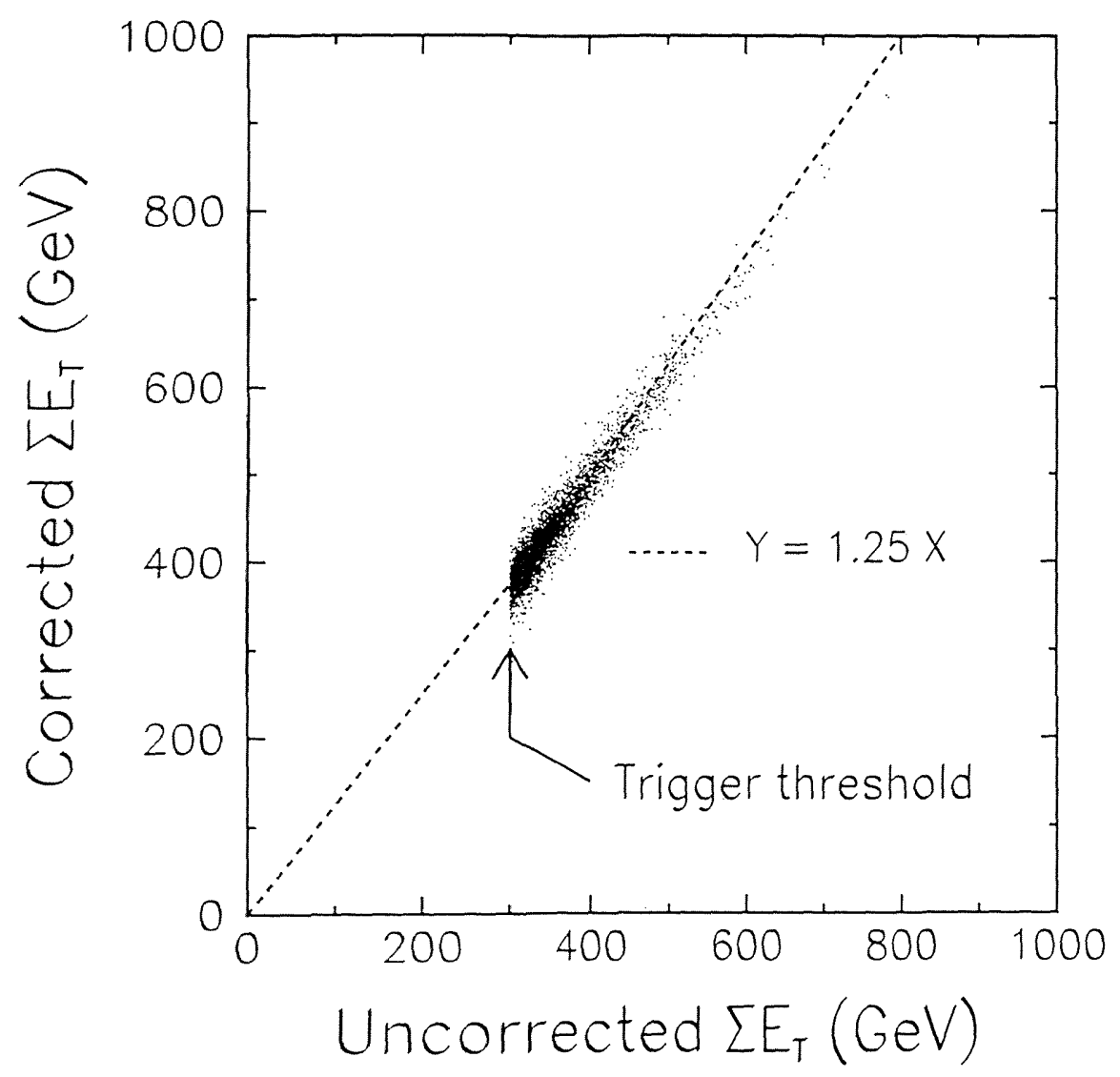

Figure 3.6: The scatter plots of the corrected and uncorrrected $\sum E_{T}$. 


\section{Chapter 4}

\section{Theoretical Predictions}

To illustrate the use of our multijet variables we will present and discuss various predictions for the distribution of multijet events in the multijet parameter space. In particular we will consider two-jet, three-jet, four-jet, and five-jet events produced at the Fermilab Proton-Antiproton Collider operating at a center of mass energy of $1.8 \mathrm{TeV}$, and compare predictions obtained from: (a) the HERWIG [22] QCD parton shower Monte Carlo program, (b) the NJETS [4] LO QCD $2 \rightarrow N$ matrix element Monte Carlo program, and (c) a model in which events are distributed uniformly over the available $\mathrm{N}$-body phase-space.

\subsection{HERWIG parton shower Monte Carlo calcu- lation}

\subsubsection{HERIWG}

HERWIG [22] is a QCD parton shower Monte Carlo program that includes both initialand final-state gluon radiation. HERWIG predictions can be thought of as LO QCD $2 \rightarrow 2$ predictions with gluon radiation and QCD jet evolution in which soft gluon interference is implemented via angular ordering. The HERWIG Monte Carlo program also includes color coherence of the initial- and final-state hard partons, backward evolution of initial-state partons including interference, hadronization of jets via nonperturbative gluon splitting, and an underlying event. We have used version 5.6 of the HERWIG Monte Carlo program, and defined jets by using a cone algorithm with 
a cone radius $R_{0}=0.7$. With this choice of cone radius we are effectively requiring that the minimum separation between jets $\Delta R_{\min }=0.9$, which is well matched to the explicit requirement $\Delta R>0.9$ described earlier.

After using a cone algorithm to define jets we use a simple detector simulation (described later) that modifies the jet energies with a Gaussian resolution function with

$$
\sigma_{E}=0.1 E
$$

This is similar to the jet energy resolution function reported by the CDF collaboration [1].

In our HERWIG calculations we have used the CTEQ1M [23] structure functions and the scale $\mathrm{Q}^{2}=\mathrm{stu} / 2\left(\mathrm{~s}^{2}+\mathrm{u}^{2}+\mathrm{t}^{2}\right)$. HERWIG generates $2 \rightarrow 2$ processes above a specified $p_{T}^{\text {hard }}$ where $p_{T}^{\text {hard }}$ is the $p_{T}$ of the outgoing partons from the hard scatter before any radiation has occurred. We have set the minimum $p_{T}^{\text {hard }}$ to $60 \mathrm{GeV} / c$. Finally, the HERWIG Monte Carlo distributions discussed in this paper are inclusive. Hence, for a given jet multiplicity $N$, the generated events contribute to the distributions if they have at least $\mathrm{N}$ jets that pass the jet requirements. If there are more than $\mathrm{N}$ jets in a generated event, the multijet system is defined using the $\mathrm{N}$ highest $E_{T}$ jets.

\subsubsection{CDF Detector Simulation}

The observed energy depositions in the detector are on average less than the true energies of the associated particles for a variety of reasons:

- The calorimeter response to low-energy charged pions is nonlinear.

- The radius of curvature of charged particles with $p_{T}<400 \mathrm{MeV} / c$ in the CDF magnetic field is such that they do not reach the calorimeter.

- The energy of particles showering in uninstrumented regions of the calorimeter is fully or partially lost (for example at the $\phi$ boundaries between calorimeter modules in the central region, or at the $\eta$ boundaries between two halves of the central calorimeter). 
- Energy taken away by neutrinos is not detected in the calorimeter, and only a small fraction of the energy of muons with $p_{T}>>2 \mathrm{GeV} / c$ is detected.

The detector simulation program extrapolates the final-state particle trajectories through the magnetic field to the calorimeter cells. The average calorimeter responses and resolution for charged pions, photons, electrons, and muons have been parameterized and tuned to reproduce:

(1) test-beam measurements for particles with momenta from a few $\mathrm{GeV} / c$ up to about $200 \mathrm{GeV} / \mathrm{c}$.

(2) studies of isolated charged particles produced in proton-antiproton collisions.

The simulation includes the variation of response across boundaries between calorimeter cells, zero response in uninstrumented regions, calorimeter non-linearities, and the observed distribution of vertex positions about the mean position at the center of the detector.

\subsection{NJETS QCD matrix element calculation}

The NJETS Monte Carlo program [4] provides parton-level predictions based on the LO QCD $2 \rightarrow N$ matrix elements. We have used the KMRSD0 structure function parameterization's [24] with the renormalization scale chosen to be the average $p_{T}$ of the outgoing partons. NJETS does not use a parton fragmentation model. Jet definitions and selection cuts are therefore applied to the final state partons. To enable a direct comparison between NJETS and HERWIG predictions we have smeared the final state parton energies in our NJETS calculations with the jet energy resolution function described above.

\subsection{Phase-Space Model}

We have used the GENBOD phase-space generator [25] to generate samples of Monte Carlo events for which the multijet systems uniformly populate the $\mathrm{N}$-body phasespace. These phase-space Monte Carlo events were generated with single-jet masses 
distributed according to the single-jet mass distribution predicted by the HERWIG Monte Carlo program. In addition, the multijet mass distributions were generated according to the corresponding distributions obtained from the HERWIG Monte Carlo calculation. Comparisons between the resulting phase-space model distributions and the corresponding HERWIG and NJETS Monte Carlo distributions help us to understand which multijet parameters are most sensitive to the behaviour of QCD multijet matrix elements. 


\section{Chapter 5}

\section{Analysis}

\subsection{Multijet Variables}

In our analysis observed jets are thought of as massive objects. Using the four momentum measured with the calorimeters the jet mass $m_{j}$ can be defined by

$$
m_{j} \equiv \sqrt{E_{j}^{2}-P_{j}^{2}}
$$

where $E_{j}$ and $P_{j}$ are the jet energy and the jet momentum. To completely describe a system of $\mathrm{N}$ massive bodies, we need to specify $4 \mathrm{~N}$ independent parameters. The topological parameters used in this analysis are defined in the $\mathrm{N}$-jet center-of-mass system (CMS), and the number of independent parameters is reduced to $4 \mathrm{~N}-3$ by the momentum conservation. Furthermore, the $\mathrm{N}$-jet system can be rotated about the incoming beam direction without losing any interesting information. Hence, we need only specify $(4 \mathrm{~N}-4)$ parameters. We will take these parameters to be the $\mathrm{N}$-jet mass and the $(4 \mathrm{~N}-5)$ dimensionless variables introduced and discussed in Ref.[26]. In choosing a set of multijet variables the criteria to be taken into account are multijet variables:

(i) that span the multijet parameter space,

(ii) make it simple to interpret the observed event distributions within the framework of perturbative QCD, and

(iii) make it is easy to compare the characteristics of events having $N$ jets with characteristics of events having for example $(\mathrm{N}+1)$ jets. 
Our $(4 \mathrm{~N}-5)$ multijet variables will provide a simple framework within which the properties of multijet events can be compared with QCD predictions. We will compare the observed distributions of these variables with corresponding distributions on QCD predictions and phase-space predictions. We begin by defining an average beam direction which will be used to define several multijet angular variables. Following that sets of three-, four-, and five-jet variables will be discussed.

\subsubsection{Average Beam Direction}

We will use the incoming beam direction as a reference in defining several angular variables. In defining the variables, it must be remembered that an $\mathrm{N}$-jet system will always be produced together with a spectator system, and the incoming partons will not be collinear in the $\mathrm{N}$-body rest frame. Hence, the following the convention of Collins and Soper [27], we will define an average beam direction $\vec{P}_{A V}$ as follows:

$$
\vec{P}_{A V} \equiv \vec{P}_{1}-\vec{P}_{2}
$$

where $\vec{P}_{1}$ and $\vec{P}_{2}$ are the momentum of the incoming particles 1 and 2 in the $N$-jet rest frame, and the incoming particle 1 is defined as the incoming interacting particle with the highest energy in the laboratory frame.

\subsubsection{Three-jet Variables}

In the standard three-jet analysis used by the UA1 collaboration[9], and later by CDF[14] and D0[15] collaborations, five variables are chosen that specify the system of three massless particles in the three-body rest frame. We have taken the first of these variables to be the three-jet mass $\left(m_{3 J}\right)$. Four additional dimensionless variables are defined that, together with $m_{3 J}$, span the three-body parameter space. In defining the three-jet parameters it is traditional to label the outgoing jets 3, 4, and 5, and order the objects according to their energies such that:

$$
E_{3}>E_{4}>E_{5}
$$

where $E_{j}$ is the energy of jet $j$ in the three-body rest frame. The traditional three-jet variables employed are $X_{3}, X_{4}, \cos \theta_{3}$, and $\psi_{3}$ : 
(i) $X_{3}$, the leading-jet energy fraction, normalized:

$$
X_{3} \equiv \frac{2 E_{3}}{E_{3}+E_{4}+E_{5}}=\frac{2 E_{3}}{m_{3 J}} .
$$

(ii) $X_{4}$, the next-to-leading-jet energy fraction, normalized:

$$
X_{4} \equiv \frac{2 E_{4}}{E_{3}+E_{4}+E_{5}}=\frac{2 E_{4}}{m_{3 J}} \text {. }
$$

Historically, R. H. Dalitz introduced a two-dimensional plot (Dalitz plot) which span the three-body parameter space in $1 \rightarrow 3$ or $2 \rightarrow 3$ decay processes using two independent variables (so-called "Dalitz variables") [28]. $X_{3}$ and $X_{4}$ are sometime taken and used as Dalitz variables. Note for three massless bodies momentum conservation restricts ranges of the Dalitz variables $X_{3}$ and $X_{4}$ :

$$
\frac{2}{3}<X_{3}<1
$$

and

$$
\frac{1}{2}<X_{4}<1
$$

(iii) $\cos \theta_{3}$, defined in the three-jet rest frame as the cosine of the leading-jet scattering angle (see Figure 5.1) :

$$
\cos \theta_{3} \equiv \frac{\vec{P}_{A V} \cdot \vec{P}_{3}}{\left|\vec{P}_{A V}\right|\left|\vec{P}_{3}\right|}
$$

(iv) $\psi_{3}$, defined in the three-jet rest frame as the angle between the three-jet plane and the plane containing jet 3 (the leading jet) and the average beam direction (see Figure 5.1):

$$
\cos \psi_{3} \equiv \frac{\left(\vec{P}_{3} \times \vec{P}_{A V}\right) \cdot\left(\vec{P}_{4} \times \vec{P}_{5}\right)}{\left|\vec{P}_{3} \times \vec{P}_{A V}\right|\left|\vec{P}_{4} \times \vec{P}_{5}\right|}
$$

To prepare for the analysis of events with more than three jets, we now wish to extend the three-jet variables to describe a system of three massive particles in the three-body rest frame. To do this, we must specify additional three parameters, which we take to be the single-jet mass $\left(m_{j}\right)$ divided by the three-jet mass: 
(v) $f_{3}$, the leading-jet mass-fraction: mass:

$$
f_{3} \equiv \frac{m_{3}}{m_{3 J}}
$$

(vi) $f_{4}$, the next-to-leading jet mass-fraction: three-body mass:

$$
f_{4} \equiv \frac{m_{4}}{m_{3 J}}
$$

(vii) $f_{5}$, the third-to-leading jet mass fraction:

$$
f_{5} \equiv \frac{m_{5}}{m_{3 J}}
$$

where the single-jet masses $m_{j}$ are defined by Equation (5.1). We conclude by noting that we have defined a set of eight variables that specify a three-jet system in the three-body rest frame:

- $\left(m_{3 J}, X_{3}, X_{4}, \cos \theta_{3} \psi_{3}, f_{3}, f_{4} \text {, and } f_{5}\right)_{3 \text {-body system }}$

\subsubsection{Four-jet Variables}

To completely describe a system of four jets in the four-body rest frame, we must specify twelve variables. We will choose the four-jet mass $m_{4 J}$ and eleven dimensionless variables that span the four-body parameter space. We have chosen a set of four-jet variables that, for four-jet configurations that approach a three-body topology, reduce to the three-jet system. This will make it possible to compare the characteristics of four-jet events with the corresponding characteristics of three-jet events.

The four-jet variables are shown schematically in Figures 5.2-(a) and -(b). We begin by reducing the four-jet system to a three-body system by combining the two jets with the lowest two-jet mass. We will label the two jets we combine $A$ and $B$ with $E_{A}>E_{B}$, where $E_{A}$ and $E_{B}$ are the jet energies in the four-jet rest frame. The resulting threebody system can be completely specified using our three-jet variables: $X_{3^{\prime}}, X_{4^{\prime}}, \cos \theta_{3^{\prime}}$, $\psi_{3^{\prime}}, f_{3^{\prime}}, f_{4^{\prime}}$ and $f_{5^{\prime}}$. Note that we order the three bodies in the three-body rest-frame so that:

$$
E_{3^{\prime}}>E_{4^{\prime}}>E_{5^{\prime}},
$$


and use a nomenclature in which primed labels denote objects after two jets have been combined. Hence, one of the three primed objects will be the two-jet system $(A B)$. Explicitly, the four-jet variables are defined:

(i) $X_{3^{\prime}}$, the fraction of the three-body energy taken by the leading object, normalized:

$$
X_{3^{\prime}} \equiv \frac{2 E_{3^{\prime}}}{E_{3^{\prime}}+E_{4^{\prime}}+E_{5^{\prime}}}=\frac{2 E_{3^{\prime}}}{m_{4 J}} .
$$

(ii) $X_{4^{\prime}}$, the fraction of the three-body energy taken by the next-to-leading object, normalized:

$$
X_{4^{\prime}} \equiv \frac{2 E_{4^{\prime}}}{E_{3^{\prime}}+E_{4^{\prime}}+E_{5^{\prime}}}=\frac{2 E_{4^{\prime}}}{m_{4 J}} .
$$

(iii) $\cos \theta_{3^{\prime}}$, the cosine of the leading-body scattering angle:

$$
\cos \theta_{3^{\prime}} \equiv \frac{\vec{P}_{A V} \cdot \vec{P}_{3^{\prime}}}{\left|\vec{P}_{A V}\right|\left|\vec{P}_{3^{\prime}}\right|}
$$

(iv) $\psi_{3^{\prime}}$, the angle between the three-body plane and the plane containing object $3^{\prime}$ (the leading body) and the average beam direction:

$$
\cos \psi_{3^{\prime}} \equiv \frac{\left(\vec{P}_{3^{\prime}} \times \vec{P}_{A V}\right) \cdot\left(\vec{P}_{4^{\prime}} \times \vec{P}_{5^{\prime}}\right)}{\left|\vec{P}_{3^{\prime}} \times \vec{P}_{A V}\right|\left|\vec{P}_{4^{\prime}} \times \vec{P}_{5^{\prime}}\right|}
$$

(v) $f_{3^{\prime}}$, the mass of the leading object divided by the four-jet mass:

$$
f_{3^{\prime}} \equiv \frac{m_{3^{\prime}}}{m_{4 J}}
$$

(vi) $f_{4^{\prime}}$, the mass of the next-to-leading object divided by the four-jet mass:

$$
f_{4^{\prime}} \equiv \frac{m_{4^{\prime}}}{m_{4 J}}
$$

(vii) $f_{5^{\prime}}$, the mass of the third-to-leading object divided by the four-jet mass:

$$
f_{5^{\prime}} \equiv \frac{m_{5^{\prime}}}{m_{4 J}}
$$

To complete our description of the four-jet system we must now specify four additional parameters that describe the two-jet $(A B)$-system. To describe the $(A B)$-system we choose: 
(i) $f_{A}$, the mass of jet $A$ divided by the four-jet mass:

$$
f_{A} \equiv \frac{m_{A}}{m_{4 J}}
$$

(ii) $f_{B}$, the mass of jet $B$ divided by the four-jet mass:

$$
f_{B} \equiv \frac{m_{B}}{m_{4 J}}
$$

(iii) $X_{A}$, defined in the four-jet rest-frame as the fraction of the energy of the $(A B)$ system taken by the leading jet:

$$
X_{A} \equiv \frac{E_{A}}{E_{A}+E_{B}}
$$

(iv) $\psi_{A B}^{\prime}$, defined in the four-jet rest-frame as the angle between (1) the plane containing the $(A B)$-system and the average beam direction, and (2) the plane containing $A$ and $B$ (see Figure 5.2-(b)). The prime reminds us that in order to define $\psi_{A B}^{\prime}$ we have combined two jets to obtain the $(A B)$-system. Note that:

$$
\cos \psi_{A B}^{\prime} \equiv \frac{\left(\vec{P}_{A} \times \vec{P}_{B}\right) \cdot\left(\vec{P}_{A B} \times \vec{P}_{A V}\right)}{\left|\vec{P}_{A} \times \vec{P}_{B}\right|\left|\vec{P}_{A B} \times \vec{P}_{A V}\right|}
$$

We conclude by noting that we have defined a set of twelve variables that specify a four-jet system in the four-body rest frame:

- $\left(m_{3 J}, X_{3^{\prime}}, X_{4^{\prime}}, \cos \theta_{3^{\prime}} \psi_{3^{\prime}}, f_{3^{\prime}}, f_{4^{\prime}}, \text { and } f_{5^{\prime}}\right)_{3 \text {-body system }}$

- $\left(X_{A}, \psi_{A B}^{\prime}, f_{A} \text {, and } f_{B}\right)_{A+B}$ system

\subsubsection{Five-jet Variables}

To completely describe a system of five jets in the five-body rest frame, we must specify sixteen variables. We will choose the five-jet mass $m_{5 J}$ and fifteen dimensionless variables that span the five-body parameter space. We have chosen a set of five-jet variables that, for five-jet configurations that approach a four-body topology, reduce to the 
four-jet variables discussed in the previous subsection. Furthermore, for five-body configurations that approach a three-body topology, our five-jet parameters reduce to the three-body variables discussed previously. The five-jet variables are shown schematically in Figures 5.3-(a) and (b). We begin by reducing the five-jet system to a four-body system by combining the two jets with the lowest two-jet mass. We will label the two jets we combine $C$ and $D$ with $E_{C}>E_{D}$, where $E_{C}$ and $E_{D}$ are the jet energies in the five-jet rest frame. We can then further reduce the resulting four-body system to a three-body system by combining two bodies with the lowest two-body mass. We will label the two objects we combine $A^{\prime}$ and $B^{\prime}$ with $E_{A^{\prime}}>E_{B^{\prime}}$. The resulting three-body system can be completely specified using our three-jet variables: $X_{3^{\prime \prime}}, X_{4^{\prime \prime}}, \cos \theta_{3^{\prime \prime}}, \psi_{3^{\prime \prime}}$, $f_{3^{\prime \prime}}, f_{4^{\prime \prime}}$ and $f_{5^{\prime \prime}}$. Note that we order the three bodies in the three-body rest-frame such that:

$$
E_{3^{\prime \prime}}>E_{4^{\prime \prime}}>E_{5^{\prime \prime}}
$$

and use a nomenclature in which doubly primed labels denote objects after two operations in which the two bodies with the lowest two-body mass have been combined. Hence, one of the three doubly primed objects will be the $\left(A^{\prime} B^{\prime}\right)$-system. Explicitly, the five-jet variables are defined:

(i) $X_{3^{\prime \prime}}$, the fraction of the three-body energy taken by the leading object, normalized:

$$
X_{3^{\prime \prime}} \equiv \frac{2 E_{3^{\prime \prime}}}{E_{3^{\prime \prime}}+E_{4^{\prime \prime}}+E_{5^{\prime \prime}}}=\frac{2 E_{3^{\prime \prime}}}{m_{5 J}} .
$$

(ii) $X_{4^{\prime \prime}}$, the fraction of the three-body energy taken by the next-to-leading object, normalized:

$$
X_{4^{\prime \prime}} \equiv \frac{2 E_{4^{\prime \prime}}}{E_{3^{\prime \prime}}+E_{4^{\prime \prime}}+E_{5^{\prime \prime}}}=\frac{2 E_{4^{\prime \prime}}}{m_{5 J}} .
$$

(iii) $\cos \theta_{3^{\prime \prime}}$, the cosine of the leading-body scattering angle:

$$
\cos \theta_{3^{\prime \prime}} \equiv \frac{\vec{P}_{A V} \cdot \vec{P}_{3^{\prime \prime}}}{\left|\vec{P}_{A V} \| \vec{P}_{3^{\prime \prime}}\right|}
$$

(iv) $\psi_{3^{\prime \prime}}$, the angle between the three-body plane and the plane containing object $3^{\prime \prime}$ (the leading body) and the average beam direction:

$$
\cos \psi_{3^{\prime \prime}} \equiv \frac{\left(\vec{P}_{3^{\prime \prime}} \times \vec{P}_{A V}\right) \cdot\left(\vec{P}_{4^{\prime \prime}} \times \vec{P}_{5^{\prime \prime}}\right)}{\left|\vec{P}_{3^{\prime \prime}} \times \vec{P}_{A V} \| \vec{P}_{4^{\prime \prime}} \times \vec{P}_{5^{\prime \prime}}\right|}
$$


(v) $f_{3^{\prime \prime}}$, the mass of the leading object divided by the five-jet mass:

$$
f_{3^{\prime \prime}} \equiv \frac{m_{3^{\prime \prime}}}{m_{5 J}}
$$

(vi) $f_{4^{\prime \prime}}$, the mass of the next-to-leading object divided by the five-jet mass:

$$
f_{4^{\prime \prime}} \equiv \frac{m_{4^{\prime \prime}}}{m_{5 J}}
$$

(vii) $f_{5^{\prime \prime}}$, the mass of the third-to-leading object divided by the five-jet mass:

$$
f_{5^{\prime \prime}} \equiv \frac{m_{5^{\prime \prime}}}{m_{5 J}}
$$

We must now specify the intermediate four-body system. In analogy with the fourjet analysis we will do this by specifying four additional dimensionless variables that describe the $\left(A^{\prime} B^{\prime}\right)$-system. We choose $f_{A^{\prime}}, f_{B^{\prime}}, X_{A^{\prime}}$, and $\psi_{A^{\prime} B^{\prime}}^{\prime \prime}$, defined:

(i) $f_{A^{\prime}}$, the mass of object $A^{\prime}$ divided by the five-jet mass:

$$
f_{A^{\prime}} \equiv \frac{m_{A^{\prime}}}{m_{5 J}}
$$

(ii) $f_{B^{\prime}}$, the mass of object $B^{\prime}$ divided by the five-jet mass:

$$
f_{B^{\prime}} \equiv \frac{m_{B^{\prime}}}{m_{5 J}}
$$

(iii) $X_{A^{\prime}}$, defined in the five-jet rest-frame as the fraction of the energy of the $\left(A^{\prime} B^{\prime}\right)$ system taken by the leading object:

$$
X_{A^{\prime}} \equiv \frac{E_{A^{\prime}}}{E_{A^{\prime}}+E_{B^{\prime}}}
$$

(iv) $\psi_{A^{\prime} B^{\prime}}^{\prime \prime}$, defined in the five-jet rest-frame as the angle between (1) the plane containing the $\left(A^{\prime} B^{\prime}\right)$-system and the average beam direction, and (2) the plane containing $A^{\prime}$ and $B^{\prime}$ (see Figure 5.3-(b)). Note that:

$$
\cos \psi_{A^{\prime} B^{\prime}}^{\prime \prime} \equiv \frac{\left(\vec{P}_{A^{\prime}} \times \vec{P}_{B^{\prime}}\right) \cdot\left(\vec{P}_{A^{\prime} B^{\prime}} \times \vec{P}_{A V}\right)}{\left|\vec{P}_{A^{\prime}} \times \vec{P}_{B^{\prime}}\right|\left|\vec{P}_{A^{\prime} B^{\prime}} \times \vec{P}_{A V}\right|}
$$


Finally, to complete our specification of the five-jet system we must define further four variables that describe the two-body $(C D)$-system. We choose $f_{C}, f_{D}, X_{C}$, and $\psi_{C D}^{\prime \prime}$ defined:

(i) $f_{C}$, the mass of jet $C$ divided by the five-jet mass:

$$
f_{C} \equiv \frac{m_{C}}{m_{5 J}}
$$

(ii) $f_{D}$, the mass of jet $D$ divided by the five-jet mass:

$$
f_{D} \equiv \frac{m_{D}}{m_{5 J}}
$$

(iii) $X_{C}$, defined in the five-jet rest-frame as the fraction of the energy of the $(C D)$ system taken by the leading jet:

$$
X_{C} \equiv \frac{E_{C}}{E_{C}+E_{D}}
$$

(iv) $\psi_{C D}^{\prime \prime}$, defined in the five-jet rest-frame as the angle between (1) the plane containing the $(C D)$-system and the average beam direction, and (2) the plane containing $C$ and $D$ (see Figure 5.3-(b)). Note that:

$$
\cos \psi_{C D}^{\prime \prime} \equiv \frac{\left(\vec{P}_{C} \times \vec{P}_{D}\right) \cdot\left(\vec{P}_{C D} \times \vec{P}_{A V}\right)}{\left|\vec{P}_{C} \times \vec{P}_{D} \| \vec{P}_{C D} \times \vec{P}_{A V}\right|}
$$

We conclude by noting that we have defined a set of sixteen variables that specify a five-jet system in the five-body rest frame:

- $\left(m_{3 J}, X_{3^{\prime \prime}}, X_{4^{\prime \prime}}, \cos \theta_{3^{\prime \prime}} \psi_{3^{\prime \prime}}, f_{3^{\prime \prime}}, f_{4^{\prime \prime}}, \text { and } f_{5^{\prime \prime}}\right)_{3 \text {-body system }}$

- $\left(X_{A^{\prime}}, \psi_{A^{\prime} B^{\prime}}^{\prime \prime}, f_{A^{\prime}}\right.$, and $\left.f_{B^{\prime}}\right){ }_{A^{\prime}+B^{\prime} \text { system }}$

- $\left(X_{C}, \psi_{C D}^{\prime \prime}, f_{C} \text {, and } f_{D}\right)_{C+D \text { system }}$

\subsubsection{Generalization to Events with Six or More Jets}

A list of the multijet variables described in the preceeding subsections is given in Table 5.1. The extension of the variables to describe multijet systems with more than 


\begin{tabular}{|c|c|c|c|}
\hline \hline Three-jet & Four-jet & Five-jet & Six-jet \\
\hline$m_{3 J}$ & $m_{4 J}$ & $m_{5 J}$ & $m_{6 J}$ \\
$\cos \theta_{3}$ & $\cos \theta_{3^{\prime}}$ & $\cos \theta_{3^{\prime \prime}}$ & $\cos \theta_{3^{\prime \prime \prime}}$ \\
$f_{3}$ & $f_{3^{\prime}}$ & $f_{3^{\prime \prime}}$ & $f_{3^{\prime \prime \prime}}$ \\
$f_{4}$ & $f_{4^{\prime}}$ & $f_{4^{\prime \prime}}$ & $f_{4^{\prime \prime \prime}}$ \\
\hline$f_{5}$ & $f_{5^{\prime}}$ & $f_{5^{\prime \prime}}$ & $f_{5^{\prime \prime \prime}}$ \\
$\psi_{3}$ & $\psi_{3^{\prime}}$ & $\psi_{3^{\prime \prime}}$ & $\psi_{3^{\prime \prime \prime}}$ \\
$X_{3}$ & $X_{3^{\prime}}$ & $X_{3^{\prime \prime}}$ & $X_{3^{\prime \prime \prime}}$ \\
$X_{4}$ & $X_{4^{\prime}}$ & $X_{4^{\prime \prime}}$ & $X_{4^{\prime \prime \prime}}$ \\
\hline & $f_{A}$ & $f_{A^{\prime}}$ & $f_{A^{\prime \prime}}$ \\
& $f_{B}$ & $f_{B^{\prime}}$ & $f_{B^{\prime \prime}}$ \\
& $X_{A}$ & $X_{A^{\prime}}$ & $X_{A^{\prime \prime}}$ \\
& $\psi_{A B}^{\prime}$ & $\psi_{A^{\prime} B^{\prime}}^{\prime \prime}$ & $\psi_{A^{\prime \prime} B^{\prime \prime}}^{\prime \prime \prime}$ \\
\hline & & $f_{C}$ & $f_{C^{\prime}}$ \\
& & $f_{D}$ & $f_{D^{\prime}}$ \\
& & $X_{C}$ & $X_{C^{\prime}}$ \\
& & $\psi_{C D}^{\prime \prime}$ & $\psi_{C^{\prime} D^{\prime}}$ \\
& & $\psi_{C D}^{\prime \prime}$ & $\psi_{C^{\prime} D^{\prime}}^{\prime \prime}$ \\
\hline & & & $f_{E}$ \\
& & & $f_{F}$ \\
& & & $X_{E}$ \\
& & & $\psi_{E F}^{\prime \prime \prime}$ \\
\hline \hline
\end{tabular}

Table 5.1: Summary of the (4N-4) multijet variables for $N=3,4,5$, and 6 .

five jets is straight forward. As an example the variables required to describe a six-jet event are also listed in Table 5.1. In general, to describe an event containing $N$ jets we use the mass of the $\mathrm{N}$-jet system plus $(4 \mathrm{~N}-5)$ dimensionless variables. To define the dimensionless variables we proceed by reducing the $\mathrm{N}$-jet system to a three-body system. This is done in $(\mathrm{N}-3)$ steps. In each step the two bodies with the lowest two-body mass are combined by adding the two four-vectors. The resulting three-body system is described by specifying seven parameters, namely the normalized masses of the three bodies (e.g. $f_{3}, f_{4}$, and $f_{5}$ ), the Dalitz variables for the two leading bodies (e.g. $X_{3}$ and $X_{4}$ ), the cosine of the leading-body scattering angle (e.g. $\cos \theta_{3}$ ), and the angle between the three-body plane and the beam direction (e.g. $\psi_{3}$ ). To complete the description of the $\mathrm{N}$-jet system we must then specify additional four parameters for each step in which two bodies were combined. These parameters are the normalized 
masses of the two bodies (e.g. $f_{A}$ and $f_{B}$ ), the fraction of the two-body energy taken by the leading body (e.g. $X_{A}$ ), and the angle defined in the $\mathrm{N}$-jet rest-frame between the plane containing the two-body system and the beam direction and the plane defined by the two bodies (e.g $\left.\psi_{A B}^{\prime}\right)$.

\subsection{Event Topology Cuts}

We left with 21830 events with three-or-more jets, 10928 events with four-or-more jets, and 4009 events with five-or-more jets after applying the multijet event selection cuts described in Chapter 4. In this section we describe the topological requirements which are applied to restrict the $\mathrm{N}$-body parameter space to the region for which the $\sum E_{T}$ requirement is efficient and to ensure that the jets in the $\mathrm{N}$-jet sample are well measured.

\subsubsection{Leading Body Scattering Angle and Multijet Mass}

Consider an event having two jets (jet 3 and 4 ) in their center of mass system. The kinematically allowed region of the leading jet scattering angle with respect to the beam particle direction $(\theta)$ is restricted by the $\sum E_{T}$ and the multijet mass requirements as follows:

$$
\begin{aligned}
m & =E_{3}+E_{4} \\
& \simeq 2 E_{3},
\end{aligned}
$$

where $m$ is the two jet mass, and $E_{3}$ and $E_{4}$ are energies of the two jets. And

$$
\begin{aligned}
\sum E_{T} & =E_{3} \sin \theta+E_{4} \sin \theta \\
& \simeq 2 E_{3} \sin \theta \\
& \simeq m \sin \theta \\
& >420 \mathrm{GeV}
\end{aligned}
$$

Hence,

$$
|\cos \theta|<\sqrt{1-\left(\frac{420}{m}\right)^{2}}
$$


and

$$
|\cos \theta|_{\max }=\sqrt{1-\left(\frac{420}{m_{\min }}\right)^{2}} .
$$

where $m_{\min }$ is the minimum value of the two-jet mass, and $|\cos \theta|_{\max }$ is the maximum value of $|\cos \theta|$. For events with relatively low energy jets in addition to two high energy jets, Equation (5.47) provides approximately the same restriction. In Figure 5.4 the scatter plots of (a) $m_{3 J}$ versus $\left|\cos \theta_{3}\right|$, (b) $m_{4 J}$ versus $\left|\cos \theta_{3^{\prime}}\right|$, and (c) $m_{5 J}$ versus $\left|\cos \theta_{3^{\prime \prime}}\right|$ are respectively shown together with the form (5.47) as a function of $m_{N J}$ (curve). It should be noted that the expression for $|\cos \theta|_{\max }$ given by (5.48) is only strictly valid for two-jet events. Indeed a close inspection of the figures reveals that at low mass the inefficient region extends a little beyond the boundary curve. For threejet events the data are clearly suppressed above the $|\cos \theta|_{\max }$ bound (Figure 5.4-(a)). For four-jet events the calculated boundary $|\cos \theta|_{\max }$ gives a poor description of the edge of the inefficient region (Figure 5.4-(b)).

The $\sum E_{T}$ requirement also does bias the multijet mass space because always

$m_{N J}>\sum E_{T}$. We are requiring $\sum E_{T}>420 \mathrm{GeV}$, hence this requires $m_{N J}>420$ $\mathrm{GeV} / \mathrm{c}^{2}$ implicitly. Fig 5.5(a)-(c) show the observed three-, four-, and five-jet mass distributions. In the figures all of the multijet mass distributions are seen to be affected by the $\sum E_{T}$ requirement. The three-jet mass distribution has a peak at $m_{3 J} \sim 500$ $\mathrm{GeV} / c^{2}$. The four- and five-jet mass distributions have peaks at $m_{4 J} \sim 550 \mathrm{GeV} / \mathrm{c}^{2}$ and $m_{5 J} \sim 600 \mathrm{GeV} / c^{2}$ respectively.

We have required $m_{3 J}>600 \mathrm{GeV} / c^{2}$ and $\left|\cos \theta_{3}\right|<0.6$ for three-jet events and $m_{4 J}>650 \mathrm{GeV} / c^{2}$ and $\left|\cos \theta_{3^{\prime}}\right|<0.8$ for four-jet events, respectively.

The inclusive five-jet data sample has very limited statistics, and the calculated boundary $|\cos \theta|_{\max }$ for five-jet events no longer describes the edge of the inefficient region well ((Figure 5.4-(c)), therefore we have only applied the requirement of $m_{5} \mathrm{~J}>$ $750 \mathrm{GeV} / c^{2}$.

\subsubsection{Dalitz Variable}

In Figures 5.6(a)-(c) shown are the observed distributions of Dalitz variables $X_{3}, X_{3^{\prime}}$, and $X_{3^{\prime \prime}}$ after applying the requirements on $m_{N J}$ and $|\cos \theta|$ discussed above. For the 


\begin{tabular}{c|c|c}
\hline \hline Three-jet analysis & Four-jet analysis & Five-jet analysis \\
\hline$m_{3 J}<600 \mathrm{GeV} / c^{2}$ & $m_{4 J}<650 \mathrm{GeV} / c^{2}$ & $m_{5 J}<750 \mathrm{GeV} / c^{2}$ \\
$X_{3}<0.9$ & $X_{3^{\prime}}<0.9$ & - \\
$\left|\cos \theta_{3}\right|<0.6$ & $\left|\cos \theta_{3^{\prime}}\right|<0.8$ & - \\
\hline \hline
\end{tabular}

Table 5.2: Event topology cuts on three-, four-, and five-jet variables. These requirements reduce, (but do not completely eliminate), the regions of low experimental acceptance.

three- and four-jet distributions, the data are clearly suppressed above $X_{3}=0.9$. This can be thought to be affected by the jet separation requirement $\Delta R_{\min }>0.9$ based on the limitation of the jet separation efficiency by the jet clustering algorithm. We require $X_{3}<0.9$ and $X_{3^{\prime}}<0.9$ for three- and four-jet events. These requirements keep us away from two-body topologies as $X_{3}$ approaches 1 , partons 4 and 5 become collinear. These topologies are poorly defined experimentally. Furthermore the QCD predictions become less reliable as we approach the collinear singularity. For five-jet events we do not apply any requirement for $X_{3^{\prime \prime}}$.

Note that the four- and five-jet requirements discussed above reduce, but do not completely eliminate, the regions of low experimental acceptance. Given the limited statistics of the present data sample, we have chosen to tolerate some regions of low experimental acceptance and use the phase-space model predictions to understand which regions of parameter space are affected. Finally we summarize the event topology cuts described above in Table 5.2. After applying these requirements we left with 1053 events with three-or-more jets, 1298 events with four-or-more jets, and 804 events five-or-more jets (Table 3.1 ( 2 nd column)). 


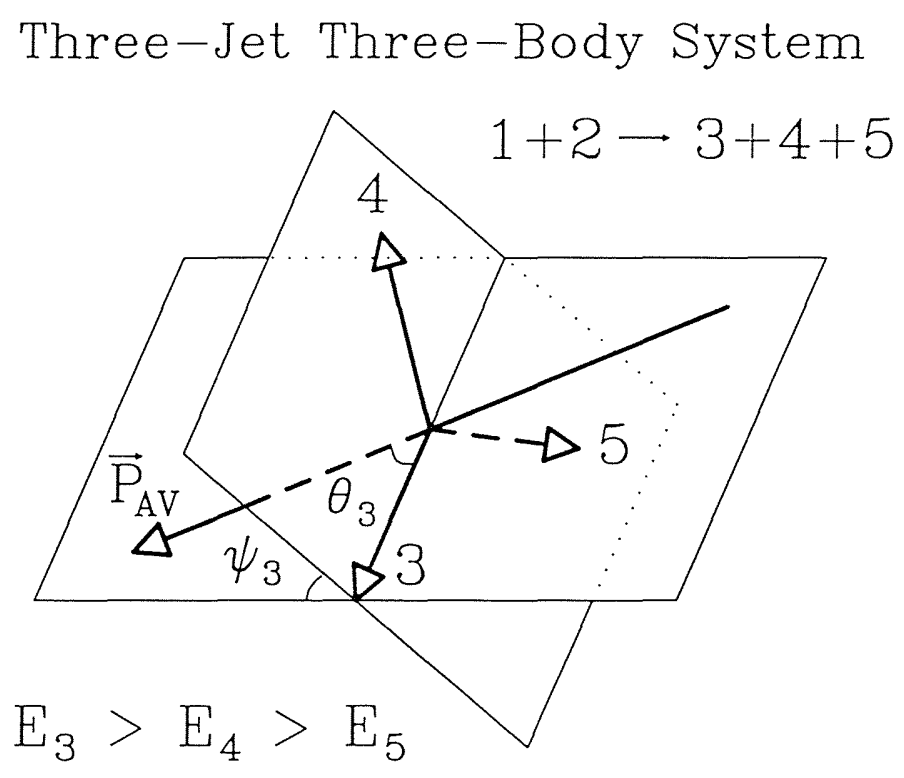

Figure 5.1: Schematic definition of angles used to describe the three-jet system in 1 three-jet rest-frame. 
(a) Four-Jet Three-Body System

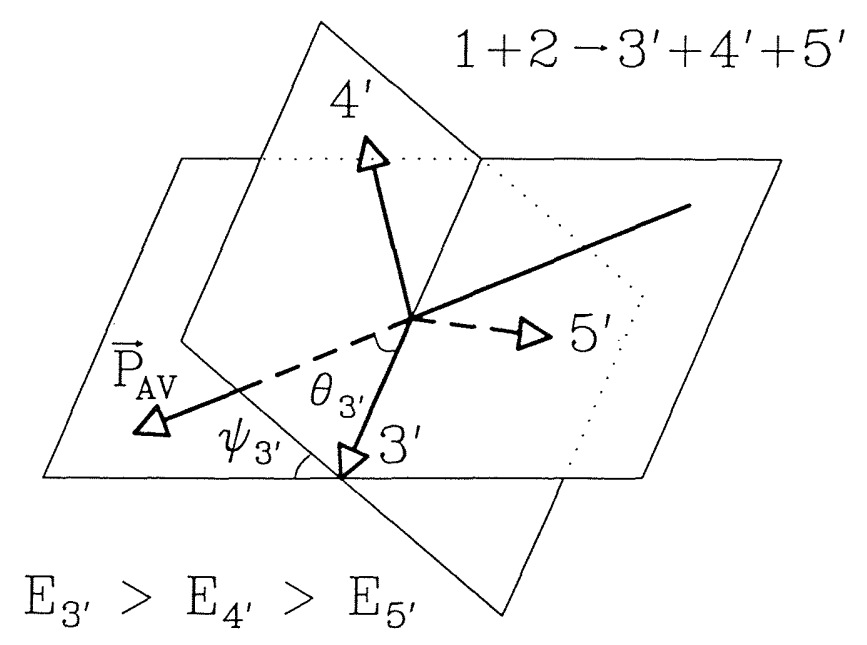

(b) Four-Jet $(A+B)$-System

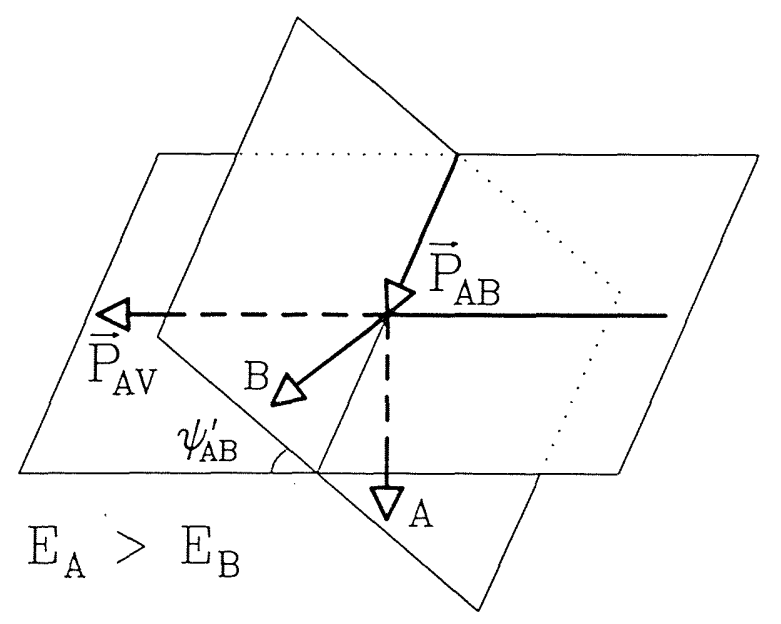

Figure 5.2: Schematic definition of angles used to describe the four-jet system in the four-jet rest-frame. 
(a) Five-Jet Three-Body System

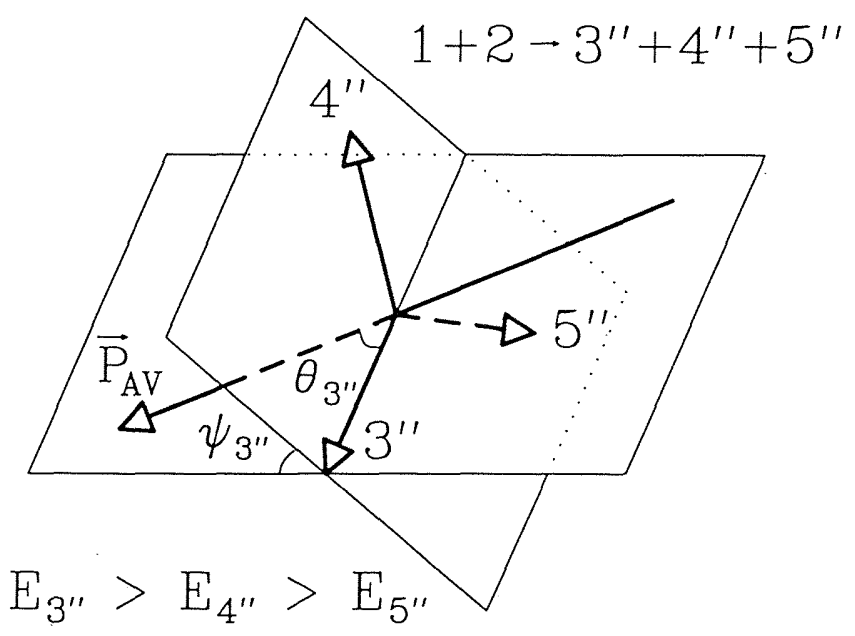

(b) Five-Jet $\left(\mathrm{A}^{\prime}+\mathrm{B}^{\prime}\right)$-System

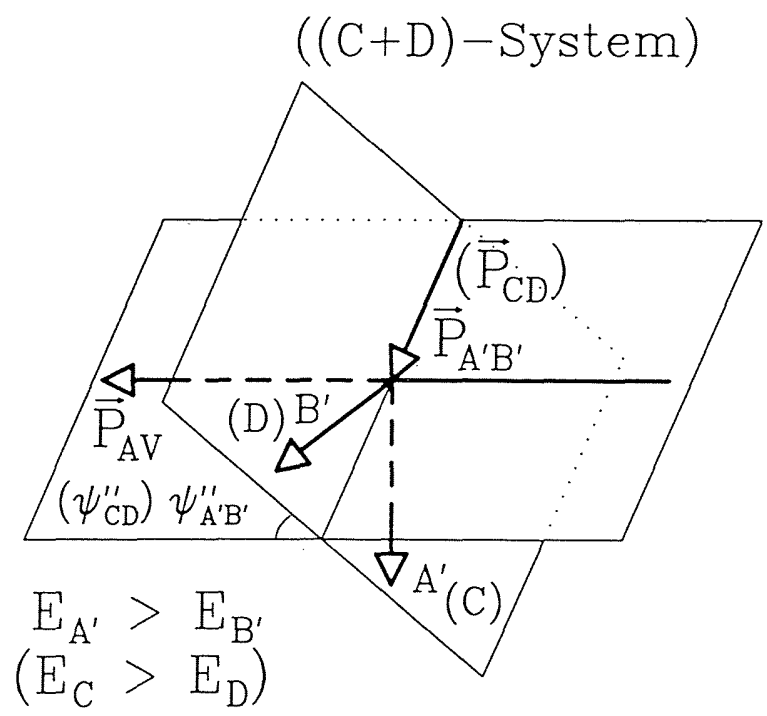

Figure 5.3: Schematic definition of angles used to describe the five-jet system in the five-jet rest-frame. 


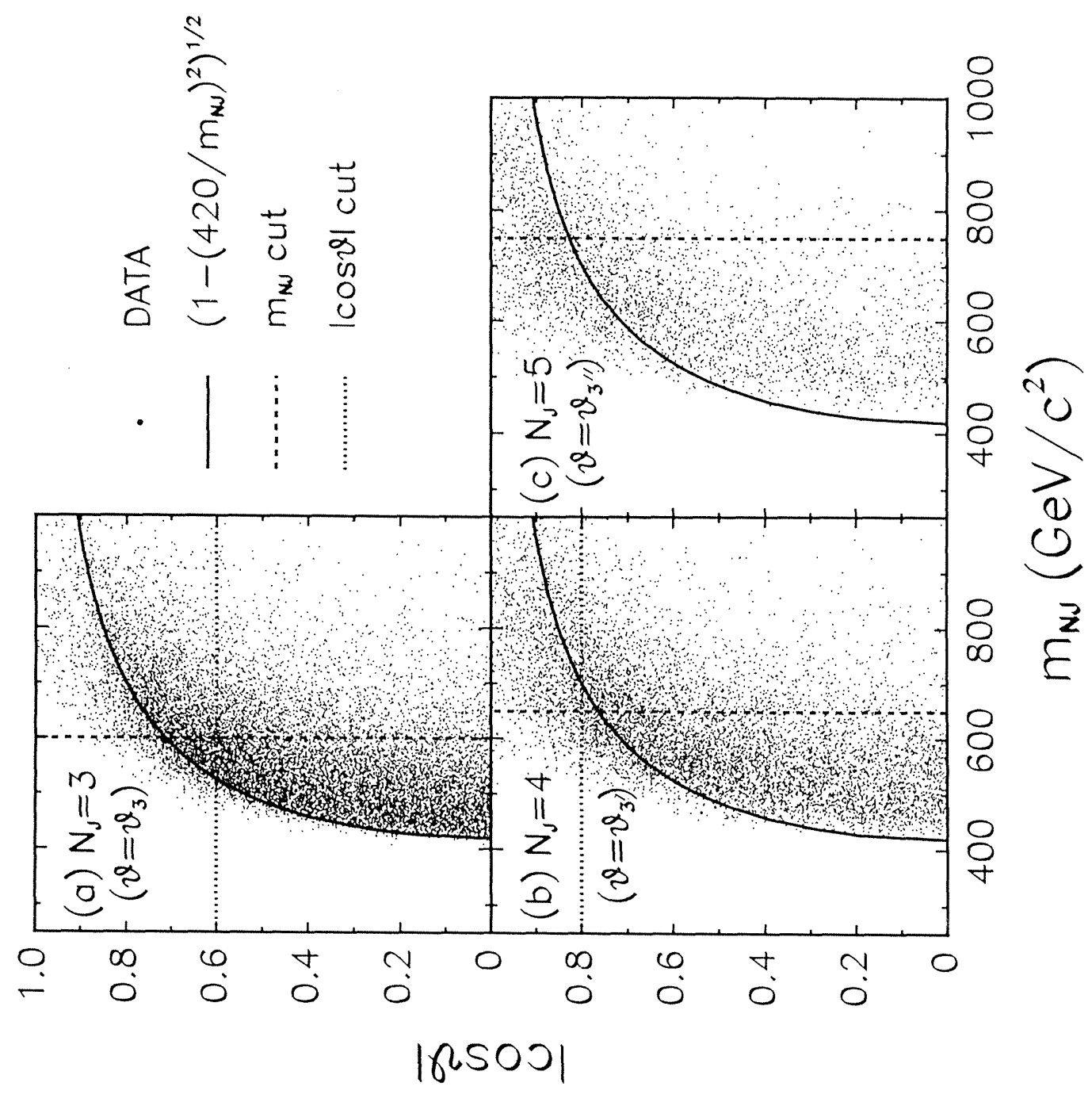

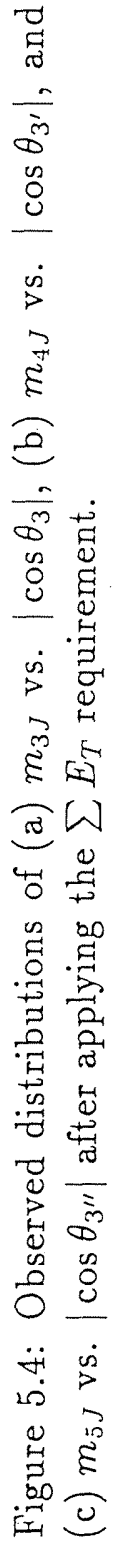



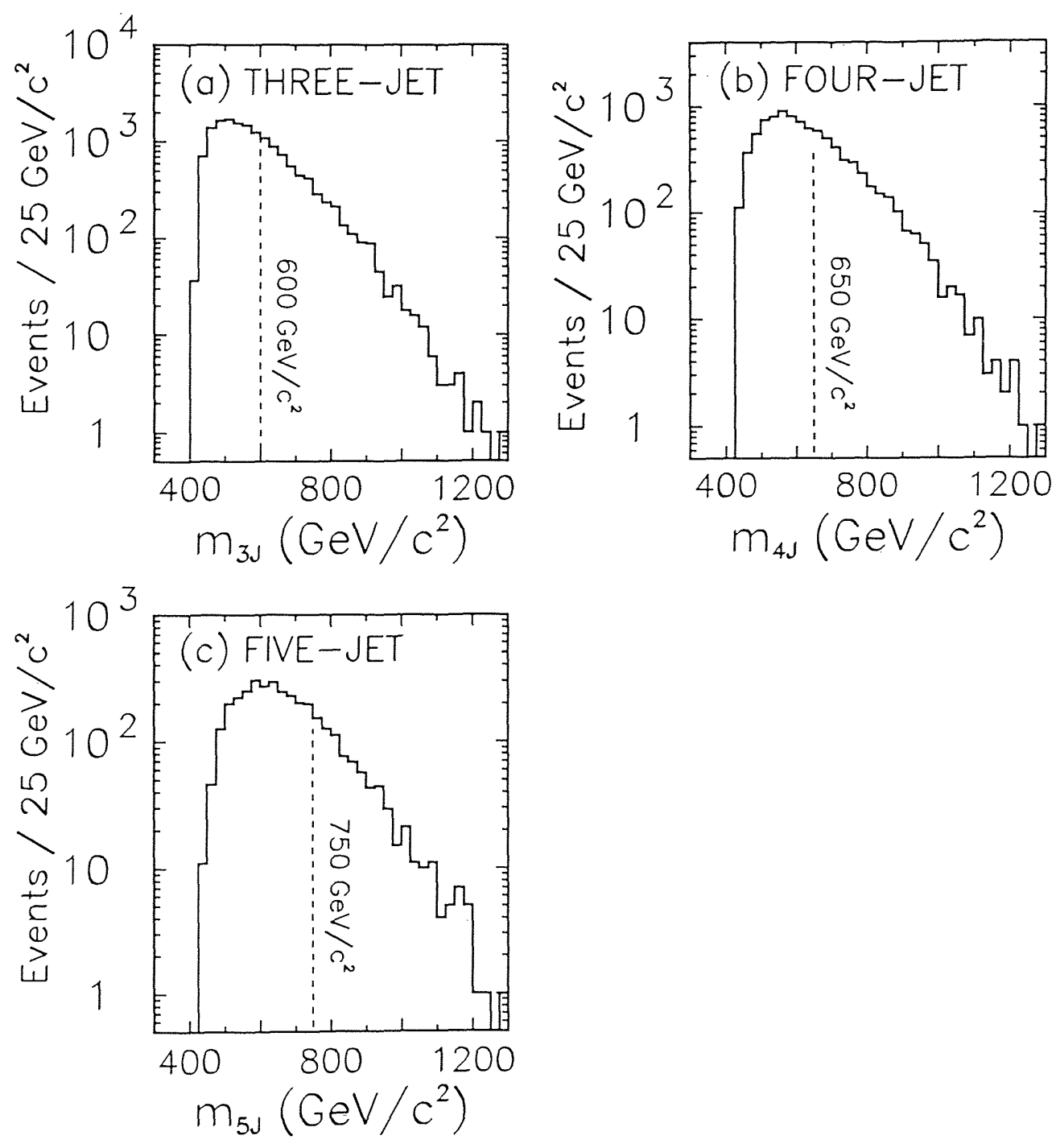

Figure 5.5: Observed distributions of inclusive multijet mass for topologies with (a) three jets, (b) four jets, and (c) five jets. 

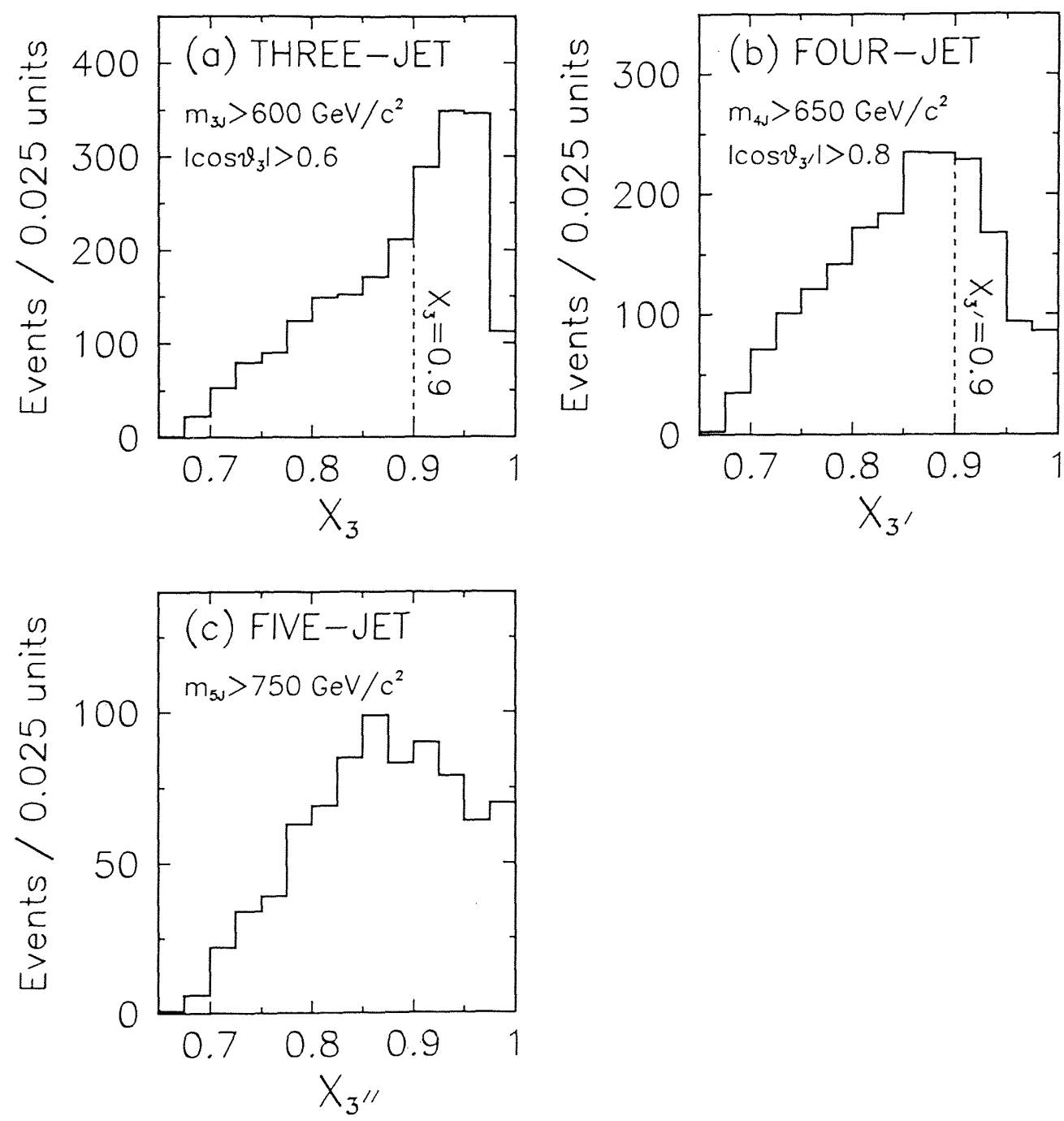

Figure 5.6: Observed distributions of Dalitz variables: (a) $X_{3^{\prime}}$, (b) $X_{3^{\prime \prime}}$, and (c) $X_{3^{\prime \prime}}$ after applying the requirements on $m_{N J}$ and $|\cos \theta|$ variables. 


\section{Chapter 6}

\section{Results and Discussion}

In the previous chapter we have defined sets of three-, four-, and five-jet variables which span the multijet parameter space in the multijet rest frame. We have applied a number of kinematical requirements to restrict the $\mathrm{N}$-body parameter space to the region for which the $\sum E_{T}$ requirement is efficient and to ensure that the jets in the $\mathrm{N}$-jet sample are well measured.

In this chapter the observed distributions of the multijet variables will be shown and compared with the QCD expectations and the phase-space model calculations. We wish to compare the shapes of the measured distributions with the predictions. Therefore, all of the distributions shown and discussed in this chapter have been normalized to unity.

We begin by comparing the observed multijet mass distributions with the QCD predictions (Section 6.1). Following that the observed distributions of the three-body variables and the two-body variables will be compared with the predictions (Sections 6.2 and 6.3). Finally we will show a result of a $\chi^{2}$ test which quantitatively provides the levels of agreement between the shapes of the observed distributions and the predicted distributions (Section 6.4).

\subsection{Multijet-mass Distributions}

All the multijet variables have been defined using the four momenta of the outgoing jets. Except for the multijet mass variables all of the multijet variables are dimensionless. Therefore the multijet masses can be thought to be most sensitive to the precision of 
the measurement of jet energies and momenta among all the multijet variables.

The CDF collaboration have reported HERWIG and NJETS QCD predictions give a good description of the shapes of the observed multijet mass distributions for exclusive samples of high-mass multijet events (1995) [1]. In Figure 6.1-(a), -(b), and -(c) both the HERWIG and NJETS predictions are shown to give good descriptions of the shapes of the inclusive $m_{3 J}, m_{4 J}$, and $m_{5 J}$ distributions for the high-mass multijet samples. Note that over the limited range of the present data sample, to a good approximation the $m_{N J}$ distributions are falling exponentially with increasing mass. The shapes of the distributions are almost independent of $N$ and are well described by an exponentially falling distribution. We have fitted the multijet mass distributions with an exponential function. The fit results are listed in Table 6.1.

\begin{tabular}{ccc}
\hline \hline topology & slope & fit $\chi^{2} /$ n.d.f. \\
\hline 3-jet & $-0.0136 \pm 0.0001$ & $6.1 / 6$ \\
4-jet & $-0.0124 \pm 0.0004$ & $5.9 / 5$ \\
5-jet & $-0.0089 \pm 0.0004$ & $4.3 / 6$ \\
\hline \hline
\end{tabular}

Table 6.1: Fit results from the exponential fits to the multijet mass distributions.

\subsection{Three-Body Distributions}

In this section we will compare the distributions of the three-body variables for three-, four-, and five-jet events with the QCD and the phase-space model predictions.

\subsubsection{Three-Body Dalitz Distributions}

We begin by considering the inclusive three-jet Dalitz distributions. Event populations in the $\left(X_{3}, X_{4}\right)$-plane are shown in Figure 6.2 for (a) data, (b) NJETS, (c) HERWIG, and (d) phase-space model predictions. The phase-space population is uniform over the kinematically allowed region. Neither the data nor the QCD predictions exhibit large density variations in the $\left(X_{3}, X_{4}\right)$-plane in the region of interest $\left(X_{3}<0.9\right)$, although with the relatively high statistical precision of the NJETS predictions the tendency for 
the predicted event density to increase as $X_{4} \rightarrow 1$ (and hence $X_{5} \rightarrow 0$ ) is apparent. The observed $X_{3}$ distribution is compared with phase-space model and QCD predictions in Figure 6.3(a). The corresponding comparisons for the $X_{4}$ distribution are shown in Figure 6.3(b). The HERWIG and NJETS predictions give reasonable descriptions of the observed distributions. Note that the observed distributions are not very different from the phase-space model predictions.

We next consider the inclusive four-jet distributions. Event populations in the $\left(X_{3^{\prime}}, X_{4^{\prime}}\right)$-plane are shown in Figure 6.4 for (a) data, (b) NJETS, (c) HERWIG, and (d) phase-space model predictions. The phase-space population is not uniform over the kinematically allowed region. Care must therefore be taken in interpreting the distributions. The data and the QCD predictions exhibit a more uniform event density over the $\left(X_{3^{\prime}}, X_{4^{\prime}}\right)$-plane. The observed $X_{3^{\prime}}$ distribution is compared with phase-space model and QCD predictions in Figure 6.5(a). The corresponding comparisons for the $X_{4^{\prime}}$ distribution are shown in Figure 6.5(b). The HERWIG and NJETS predictions give reasonable descriptions of the observed distributions. Note that compared to the phase-space model predictions, the data and $\mathrm{QCD}$ predictions prefer topologies with $X_{3^{\prime}} \rightarrow 1$ (for which the three-body topology approaches a two-body configuration) and $X_{4^{\prime}} \rightarrow 1$ (for which $X_{5^{\prime}} \rightarrow 0$ ).

Finally, consider the inclusive five-jet distributions. Event populations in the $\left(X_{3^{\prime \prime}}\right.$, $X_{4^{\prime \prime}}$ )-plane are shown in Figure 6.6 for (a) data, (b) NJETS, (c) HERWIG, and (d) phase-space model predictions. Again, the phase-space population is not uniform over the kinematically allowed region, and care must be taken in interpreting the distributions. The observed event population and the QCD predictions are more uniformly distributed over the $\left(X_{3^{\prime}}, X_{4^{\prime}}\right)$-plane. However, all distributions are depleted as $X_{3^{\prime \prime}} \rightarrow 1$ and $X_{4^{\prime \prime}} \rightarrow 1$. The observed $X_{3^{\prime \prime}}$ distribution is compared with phase-space model and QCD predictions in Figure 6.7(a). The corresponding comparisons for the $X_{4^{\prime \prime}}$ distribution are shown in Figure 6.7(b). The HERWIG and NJETS predictions give reasonable descriptions of the observed distributions. Note that compared to the phasespace model predictions, the data and QCD predictions prefer topologies with $X_{3^{\prime \prime}} \rightarrow 1$ and $X_{4^{\prime \prime}} \rightarrow 1$. 


\subsubsection{Three-Body Angular Distributions}

We begin by considering the inclusive three-jet angular distributions. Event populations in the $\left(\cos \theta_{3}, \psi_{3}\right)$-plane are shown in Figure 6.8 for (a) data, (b) NJETS, (c) HERWIG, and (d) phase-space model predictions. The phase-space population is approximately uniform. In contrast both the observed distribution and the QCD predictions exhibit large density variations over the $\left(\cos \theta_{3}, \psi_{3}\right)$-plane, with the event density increasing as $\left|\cos \theta_{3}\right| \rightarrow 1$ and $\psi_{3} \rightarrow 0$ or $\pi$. The increase in event rate as $\left|\cos \theta_{3}\right| \rightarrow 1$ is similar to the behaviour of the leading-jet angular distribution resulting from the $2 \rightarrow 2 \mathrm{LO}$ QCD matrix element. The increase in event rate as $\psi_{3} \rightarrow 0$ or $\pi$ reflects the preference of the three-jet matrix element for topologies which are planar. It is interesting to note that as $\cos \theta_{3} \rightarrow 1$ the NJETS calculation shows a preference for configurations with $\psi_{3} \rightarrow 0$ rather than $\pi$ and as $\cos \theta_{3} \rightarrow-1$ the NJETS calculation shows a preference for configurations with $\psi_{3} \rightarrow \pi$ rather than 0 . These preferred regions of the parameter space correspond to configurations in which jet 5 is closer to the beam direction, and therefore reflect the initial state radiation pole in the matrix element. The observed $\cos \theta_{3}$ distribution is compared with phase-space model and QCD predictions in Figure 6.9(a). The corresponding comparisons for the $\psi_{3}$ distribution are shown in Figure 6.9(b). Both HERWIG and NJETS predictions give reasonable descriptions of the observed distributions, which are very different from the phase-space model predictions. Note that the observed $\cos \theta_{3}$ distribution is also very similar to the LO prediction for $q \bar{q} \rightarrow q \bar{q}$ scattering.

Next, consider the inclusive four-jet angular distributions. Event populations in the $\left(\cos \theta_{3^{\prime}}, \psi_{3^{\prime}}\right)$-plane are shown in Figure 6.10 for (a) data, (b) NJETS, (c) HERWIG, and (d) phase-space model predictions. The phase-space population is approximately uniform. In contrast both the observed distribution and the QCD predictions exhibit large density variations over the $\left(\cos \theta_{3^{\prime}}, \psi_{3^{\prime}}\right)$-plane, with the event density increasing as $\left|\cos \theta_{3^{\prime}}\right| \rightarrow 1$ and $\psi_{3^{\prime}} \rightarrow 0$ or $\pi$. This behaviour is similar to the behaviour of the corresponding three-jet distributions. The observed $\cos \theta_{3^{\prime}}$ distribution is compared with phase-space model and QCD predictions in Figure 6.11(a). The corresponding comparisons for the $\psi_{3^{\prime}}$ distribution are shown in Figure 6.11(b). Both HERWIG and 
NJETS predictions give reasonable descriptions of the observed distributions, which are very different from the phase-space model predictions. Note that the observed $\cos \theta_{3^{\prime}}$ distribution is also very similar to the LO prediction for $q \bar{q} \rightarrow q \bar{q}$ scattering.

Finally, consider the inclusive five-jet angular distributions. Event populations in the $\left(\cos \theta_{3^{\prime \prime}} ; \psi_{3^{\prime \prime}}\right)$-plane are shown in Figure 6.12 for (a) data, (b) NJETS, (c) HERWIG, and (d) phase-space model predictions. The phase-space population is not uniform, and care must therefore be taken in interpreting the distributions. However, both the observed distribution and the QCD predictions exhibit much larger density variations over the $\left(\cos \theta_{3^{\prime \prime}}, \psi_{3^{\prime \prime}}\right)$-plane, with the event density increasing as $\left|\cos \theta_{3^{\prime \prime}}\right| \rightarrow 1$ and $\psi_{3^{\prime \prime}} \rightarrow 0$ or $\pi$. This behaviour is similar to the behaviour of the corresponding three-jet distributions. The observed $\cos \theta_{3^{\prime \prime}}$ distribution is compared with phase-space model and QCD predictions in Figure 6.13(a). The corresponding comparisons for the $\psi_{3^{\prime \prime}}$ distribution are shown in Figure 6.13(b). Both HERWIG and NJETS predictions give reasonable descriptions of the observed distributions, which are very different from the phase-space model predictions. Note that the observed $\cos \theta_{3^{\prime \prime}}$ distribution is also very similar to the $\mathrm{LO}$ prediction for $q \bar{q} \rightarrow q \bar{q}$ scattering even though there are now five jets in the final state.

\subsubsection{Single-Body Mass Distributions for Three-Body Sys- tems}

The normalized single-jet mass distributions are shown in Figure 6.14 for inclusive three-jet events. The $f_{j}$ distributions are reasonably well described by the HERWIG Monte Carlo predictions, although there is a tendency for the HERWIG fragmentation model to slightly overestimate the fraction of low-mass jets. The observed distributions peak at $f_{j} \sim 0.05$ or less. Hence, for many purposes, jets at high energy can be considered to be massless. Note that since jets are massless in the matrix element calculations, there are no NJETS predictions for the $f_{j}$ distributions.

The $f_{j^{\prime}}$ and $f_{j^{\prime \prime}}$ distributions are shown for inclusive four-jet and inclusive five-jet events in Figures 6.15 and 6.16 respectively. These exhibit a single-jet peak at low massfractions (less than 0.05), and have a long tail associated with two-jet $j^{\prime}$ systems, and two-jet or three-jet $j^{\prime \prime}$ systems. The HERWIG predictions give a good description of all 
the distributions except perhaps at very low mass fractions (less than 0.05) where there is tendency to overestimate the observed jet rate. Although the NJETS calculations do not provide predictions for the single-jet part of the $f_{j^{\prime}}$ and $f_{j^{\prime \prime}}$ distributions, they are seen to correctly predict the tail associated with multijet $j^{\prime}$ and $j^{\prime \prime}$ systems.

\subsection{Two-Body Distributions}

\subsubsection{Two-Body Energy Sharing Distributions}

The observed $X_{A}$ distribution is shown in Figures 6.17(a) and (b) to be reasonably well described by the HERWIG and NJETS predictions. To gain some insight into the shape of the phase-space model predictions for the $X_{A}$ distribution consider a system of four-massless particles labeled randomly $\mathrm{i}, \mathrm{j}, \mathrm{k}$, and l. If we define $X_{i} \equiv E_{i} /\left(E_{i}+E_{j}\right)$, then the phase-space prediction for the distribution of events as a function of $X_{i}$ is given by

$$
\frac{d \Phi_{4}}{d X} \sim-\frac{1}{X^{3}}+\frac{3}{X^{2}}-2 \quad(\text { See Appendix C })
$$

This function is already quite similar to the phase-space model prediction shown in Figure 6.17(a), which is obtained by requiring that the $(A B)$-system is the lowest mass pair, and taking account of finite single-jet masses and experimental selection requirements. The data and the QCD predictions favor a more asymmetric sharing of energy between the two jets $A$ and $B$ than predicted by the phase-space model. This presumably reflects the presence of the soft gluon radiation pole in the QCD matrix element. In Figures 6.17(c) and (d) the $X_{A^{\prime}}$ distributions are shown to be qualitatively similar to the corresponding $X_{A}$ distributions, and also similar to the corresponding $X_{C}$ distributions shown in Figures 6.17(e) and (f). In all cases the data are reasonably well described by the QCD predictions and are very different from the phase-space model predictions.

\subsubsection{Two-Body Angular Distributions}

The observed $\psi_{A B}^{\prime}$ distribution is shown in Figures 6.18(a) and (b) to be well described by the HERWIG and NJETS predictions. The phase-space model prediction is also 
approximately uniform, but underestimates the fraction of events in which the plane of the two-body system is close to the plane containing the two-body system and the beam direction $\left(\psi_{A B}^{\prime} \rightarrow 0\right.$ or $\pi$ ). This difference between phase-space model and QCD predictions presumably reflects the presence of the initial-state radiation pole in the QCD matrix element. In Figures 6.18(c) and (d) the $\psi_{A^{\prime} B^{\prime}}^{\prime \prime}$ distributions are shown to be qualitatively similar to the corresponding $\psi_{A B}^{\prime}$ distributions. The $\psi_{C D}^{\prime \prime}$ distributions shown in Figures 6.18(e) and (f) to be very similar to the phase-space model predictions. In all cases the data are well described by the QCD predictions. None of the observed distributions are very different from the phase-space model predictions, although the phase-space model calculation does underestimate the event rate as $\psi_{A B}^{\prime} \rightarrow 0$ or $\pi$, or as $\psi_{A^{\prime} B^{\prime}}^{\prime \prime} \rightarrow 0$ or $\pi$.

\subsubsection{Single-Body Mass Distributions for Two-Body Systems}

The observed $f_{A}, f_{B}, f_{A^{\prime}}, f_{B^{\prime}}, f_{C}$, and $f_{D}$ distributions are shown in Figures 6.19(a),(b), $(\mathrm{c}),(\mathrm{d}),(\mathrm{e})$, and (f) respectively to be reasonably well described by the HERWIG predictions although there is a tendency for the HERWIG predictions to overestimate the jet rate at very small single-jet masses. In all cases the distributions exhibit a single-jet mass peak at small mass fractions ( 0.02 or less). The $f_{A^{\prime}}$ and $f_{B^{\prime}}$ have a long high-mass tail which corresponds to two-jet $A^{\prime}$ and $B^{\prime}$ systems. This tail is well described by the NJETS predictions.

\section{$6.4 \chi^{2}$ Test}

In general HERWIG and NJETS QCD predictions appear to give a good first description of the observed multijet distributions, which correspond to $(4 \mathrm{~N}-4)$ variables that span the $\mathrm{N}$-body parameter space. A more quantitative assessment can be made by computing the $\chi^{2}$ per degree of freedom that characterizes the agreement between the observed distributions and the QCD predictions. 


\subsubsection{Definition of $\chi^{2}$}

Our HERWIG Monte Carlo distributions have statistical uncertainties which are comparable to those of the observed data samples. In comparing an observed distribution to a corresponding prediction, the uncertainties on both the distributions must be taken into account. Therefore we defined " $\chi$ " as follows:

$$
\chi^{2} \equiv \sum_{i} \frac{\left(X_{\text {data }}(i)-X_{M . C .}(i)\right)^{2}}{\sigma_{\text {data }}^{2}(i)+\sigma_{M . C .}^{2}(i)}
$$

where $X_{\text {data }}(i)$ ( $\left.X_{\text {M.C. }}(i)\right)$ is the content of a histogram of the observed data (the M.C. predictions $)$ in the $i$-th bin, and $\sigma_{\text {data }}(i)\left(\sigma_{M . C .}(i)\right)$ is the error for $X_{\text {data }}(i)\left(X_{M . C .}(i)\right)$. Note that we have only taken into account of statistical uncertainties in this test. The validity of this definition was studied by a Monte Carlo technique (See Appendix B).

\subsubsection{Results of the $\chi^{2}$ Test}

The $\chi^{2}$ is listed for each distribution in Table 6.2-6.4. The computed $\chi^{2}$ 's take into account only statistical uncertainties on both measured points and the QCD Monte Carlo predictions. The theoretical uncertainties on the predictions arising from choice of structure function, $Q^{2}$-scale ..etc., and the experimental uncertainties associated with the uncertainties on the jet energy scale ..etc. will be discussed in Chapter 7 . However, even in the absence of a full evaluation of the systematic uncertainties, an examination of the tables shows that NJETS provides a reasonable description of all of the observed multijet distributions except perhaps the $X_{A}$ distribution. The combined $\chi^{2}$ for the NJETS description of all of the three-jet distributions $\chi^{2} / \mathrm{NDF}=0.93(45$ degrees of freedom). The corresponding result for the four-jet distributions is $\chi^{2} / N D F$ $=1.47$ (64 degrees of freedom) if the $X_{A}$ distribution is included in the comparison, and $\chi^{2} / \mathrm{NDF}=1.13$ (56 degrees of freedom) if the $X_{A}$ distribution is not included. The result for the combined five-jet distributions is $\chi^{2} / \mathrm{NDF}=1.11$ (63 degrees of freedom). The observed distributions are described less well by the HERWIG parton shower Monte Carlo predictions, for which the $X_{4}, \psi_{3^{\prime}}$, and $\cos \theta_{3^{\prime \prime}}$ distributions have $\chi^{2} \mathrm{~s}$ significantly poorer than those for the corresponding NJETS predictions. Restricting the comparison to those distributions predicted by both the NJETS and HERWIG 
calculations (i.e. all distributions except the single-body mass fraction distributions) we find the overall $\chi^{2}$ per degree of freedom for the HERWIG comparison of the combined three-jet distributions is $\chi^{2} / \mathrm{NDF}=1.54$ (45 degrees of freedom), for the combined four-jet distributions $\chi^{2} / \mathrm{NDF}=1.64$ (64 degrees of freedom, and for the combined five-jet distributions $\chi^{2} / \mathrm{NDF}=1.36$ (63 degrees of freedom).

\subsubsection{Event Rates}

Finally we compare the measured event rate of multijet events with the corresponding predictions for each topology. The cross-section $\sigma_{N J}$ for $\mathrm{N}$-jet inclusive events is simply defined using the integrated luminosity and the number of $\mathrm{N}$-jet inclusive events $N_{\mathrm{NJ}}$ getting into multijet distributions:

$$
\sigma_{N J} \equiv \frac{N_{\mathrm{NJ}}}{\int \mathcal{L} d t}
$$

The observed cross-sections and the corresponding NJETS and HERIWG predictions are listed in Table 6.5. The errors are statistical only. Neither theoretical nor experimental uncertainties were not taken into account. Both NJETS and HERIWG are seen to underestimate the measured cross-sections for three-, four-, and five-jet inclusive events. 


\begin{tabular}{c|ccc|c}
\hline \hline \multirow{2}{*}{$\begin{array}{c}3 \text {-jet } \\
\text { variables }\end{array}$} & \multicolumn{3}{|c}{$\chi^{2} /$ n.d.f. } & \\
\cline { 2 - 4 } & NJETS-DATA & HERWIG-DATA & NJETS-HERWIG & n.d.f. \\
\hline$m_{3 J}$ & 1.27 & 1.07 & 0.41 & 6 \\
$X_{3}$ & 0.41 & 1.41 & 2.67 & 8 \\
$X_{4}$ & 1.42 & 3.66 & 4.08 & 6 \\
$\cos \theta_{3}$ & 0.95 & 0.77 & 1.04 & 11 \\
$\psi_{3}$ & 0.84 & 1.52 & 2.12 & 14 \\
$f_{3}$ & - & 2.85 & - & 9 \\
$f_{4}$ & - & 8.70 & - & 9 \\
$f_{5}$ & - & 0.14 & - & 6 \\
\hline over all & 0.93 & 1.54 & 1.99 & 45 \\
\hline \hline
\end{tabular}

Table 6.2: Statistical comparison of agreement between observed and predicted distributions of the three-jet variables. The $\chi^{2}$ s per degree of freedom are listed. In calculating the over all $\chi^{2}$ s, the single body mass fraction variables, for which NJETS does not provide the full distributions, are excluded.

\begin{tabular}{c|ccc|c}
\hline \hline \multirow{2}{*}{$\begin{array}{c}4 \text {-jet } \\
\text { variables }\end{array}$} & \multicolumn{3}{|c|}{$\chi^{2} /$ n.d.f. } & \multirow{2}{*}{ n.d.f. } \\
\cline { 2 - 4 } & NJETS-DATA & HERWIG-DATA & NJETS-HERWIG & 6 \\
$m_{4 J}$ & 0.66 & 0.45 & 0.15 & 8 \\
$X_{3^{\prime}}$ & 0.66 & 0.82 & 1.60 & 6 \\
$x_{4^{\prime}}$ & 0.40 & 0.92 & 2.36 & 15 \\
$\cos \theta_{3^{\prime}}$ & 1.70 & 1.94 & 1.87 & 14 \\
$\psi_{3^{\prime}}$ & 1.36 & 2.63 & 1.65 & 7 \\
$\psi_{A B}^{\prime}$ & 1.01 & 1.98 & 1.85 & 8 \\
$X_{A}$ & 3.80 & 1.31 & 2.06 & 11 \\
$f_{3^{\prime}}$ & - & 2.85 & - & 8 \\
$f_{4^{\prime}}$ & - & 3.42 & - & 8 \\
$f_{5^{\prime}}$ & - & 2.38 & - & 13 \\
$f_{A}$ & - & 1.85 & - & 11 \\
$f_{B}$ & - & 4.16 & - & 64 \\
\hline over all & 1.47 & 1.64 & 1.70 & \\
\hline \hline
\end{tabular}

Table 6.3: Statistical comparison of agreement between observed and predicted distributions of the four-jet variables. The $\chi^{2}$ s per degree of freedom are listed. In calculating the over all $\chi^{2}$ s, the single body mass fraction variables, for which NJETS does not provide the full distributions, are excluded. 


\begin{tabular}{c|ccc|c}
\hline \hline \multirow{2}{*}{$\begin{array}{c}5 \text {-jet } \\
\text { variables }\end{array}$} & \multicolumn{3}{|c|}{$\chi^{2} /$ n.d.f. } & \\
\cline { 2 - 4 } & NJETS-DATA & HERWIG-DATA & NJETS-HERWIG & n.d.f. \\
\hline$m_{5 J}$ & 0.52 & 0.83 & 1.57 & 8 \\
$X_{3^{\prime \prime}}$ & 1.08 & 0.66 & 1.90 & 7 \\
$X_{4^{\prime \prime}}$ & 0.42 & 0.85 & 0.72 & 6 \\
$\cos \theta_{3^{\prime \prime}}$ & 1.12 & 2.84 & 7.74 & 7 \\
$\psi_{3^{\prime \prime}}$ & 1.49 & 2.02 & 2.02 & 7 \\
$\psi_{A^{\prime} B^{\prime}}^{\prime \prime}$ & 0.95 & 1.13 & 0.35 & 7 \\
$\psi_{C D}^{\prime \prime}$ & 1.16 & 1.11 & 0.22 & 7 \\
$X_{A^{\prime}}$ & 1.76 & 2.38 & 1.96 & 7 \\
$X_{C}$ & 1.47 & 0.43 & 1.22 & 7 \\
$f_{3^{\prime \prime}}$ & - & 3.32 & - & 12 \\
$f_{4^{\prime \prime}}$ & - & 6.68 & - & 8 \\
$f_{5^{\prime \prime}}$ & - & 2.76 & - & 8 \\
$f_{C}$ & - & 1.85 & - & 10 \\
$f_{D}$ & - & 6.37 & - & 7 \\
$f_{A^{\prime}}$ & - & 3.26 & - & 12 \\
$f_{B^{\prime}}$ & - & 1.22 & - & 12 \\
\hline over all & 1.11 & 1.36 & 1.98 & 63 \\
\hline \hline
\end{tabular}

Table 6.4: Statistical comparison of agreement between observed and predicted distributions of the five-jet variables. The $\chi^{2}$ s per degree of freedom are listed. In calculating the over all $\chi^{2}$ s, the single body mass fraction variables, for which NJETS does not provide the full distributions, are excluded.

\begin{tabular}{lccc}
\hline \hline & DATA & NJETS & HERWIG \\
\hline$\sigma_{3 J}(\mathrm{pb})$ & $10.03 \pm 0.31$ & $4.88 \pm 0.04$ & $5.92 \pm 0.21$ \\
$\sigma_{4 J}(\mathrm{pb})$ & $12.36 \pm 0.34$ & $5.80 \pm 0.16$ & $6.59 \pm 0.22$ \\
$\sigma_{5 J}(\mathrm{pb})$ & $7.66 \pm 0.27$ & $2.88 \pm 0.10$ & $5.62 \pm 0.20$ \\
\hline \hline
\end{tabular}

Table 6.5: The observed and predicted cross-sections for inclusive three-jet, four-jet, and five-jet events. Errors are statistical only. 


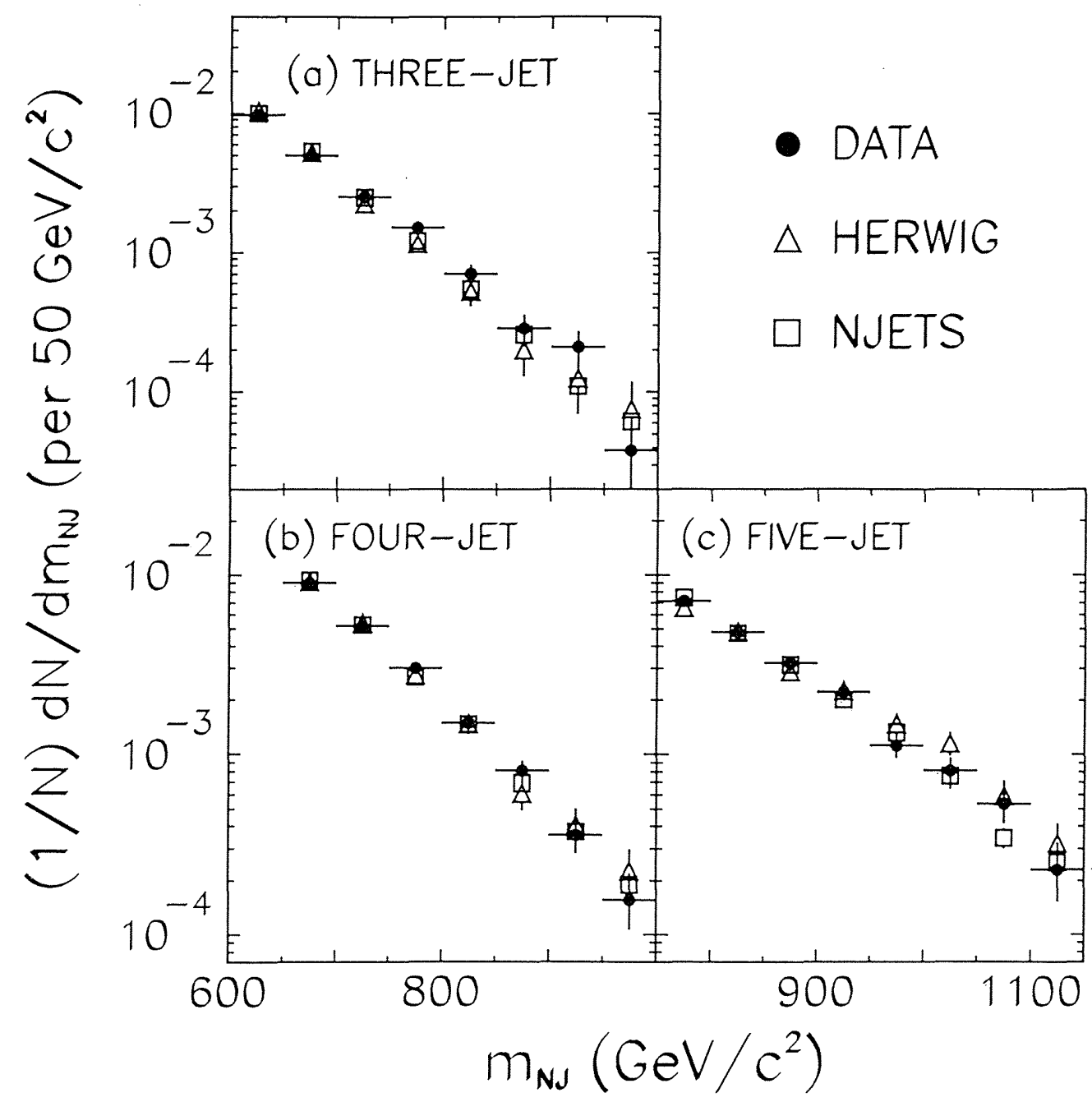

Figure 6.1: Inclusive multijet mass distributions for topologies with (a) three jets, (b) four jets, and (c) five jets. Observed distributions (points) are compared with HERWIG predictions (triangles) and NJETS predictions (squares). 

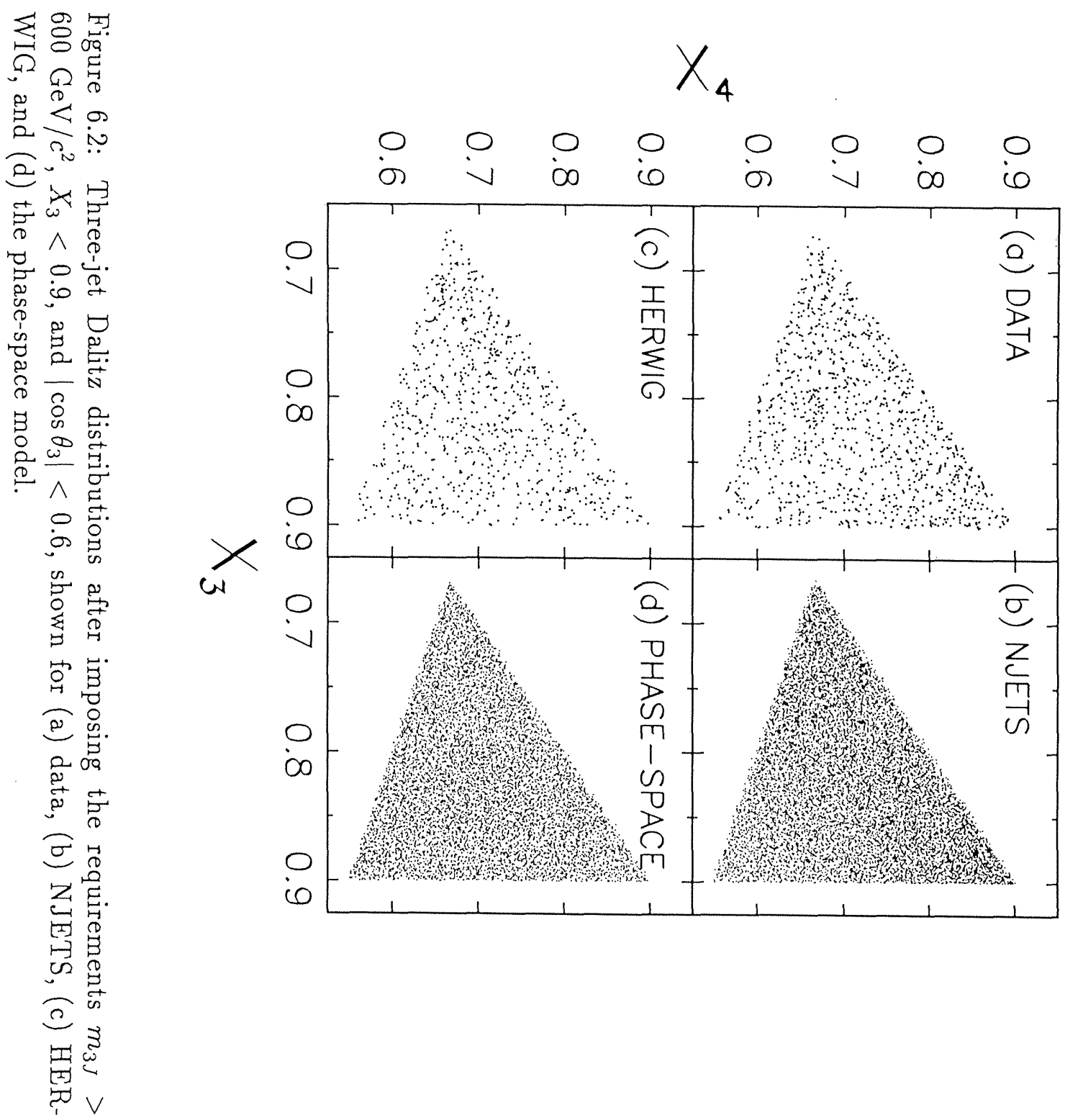

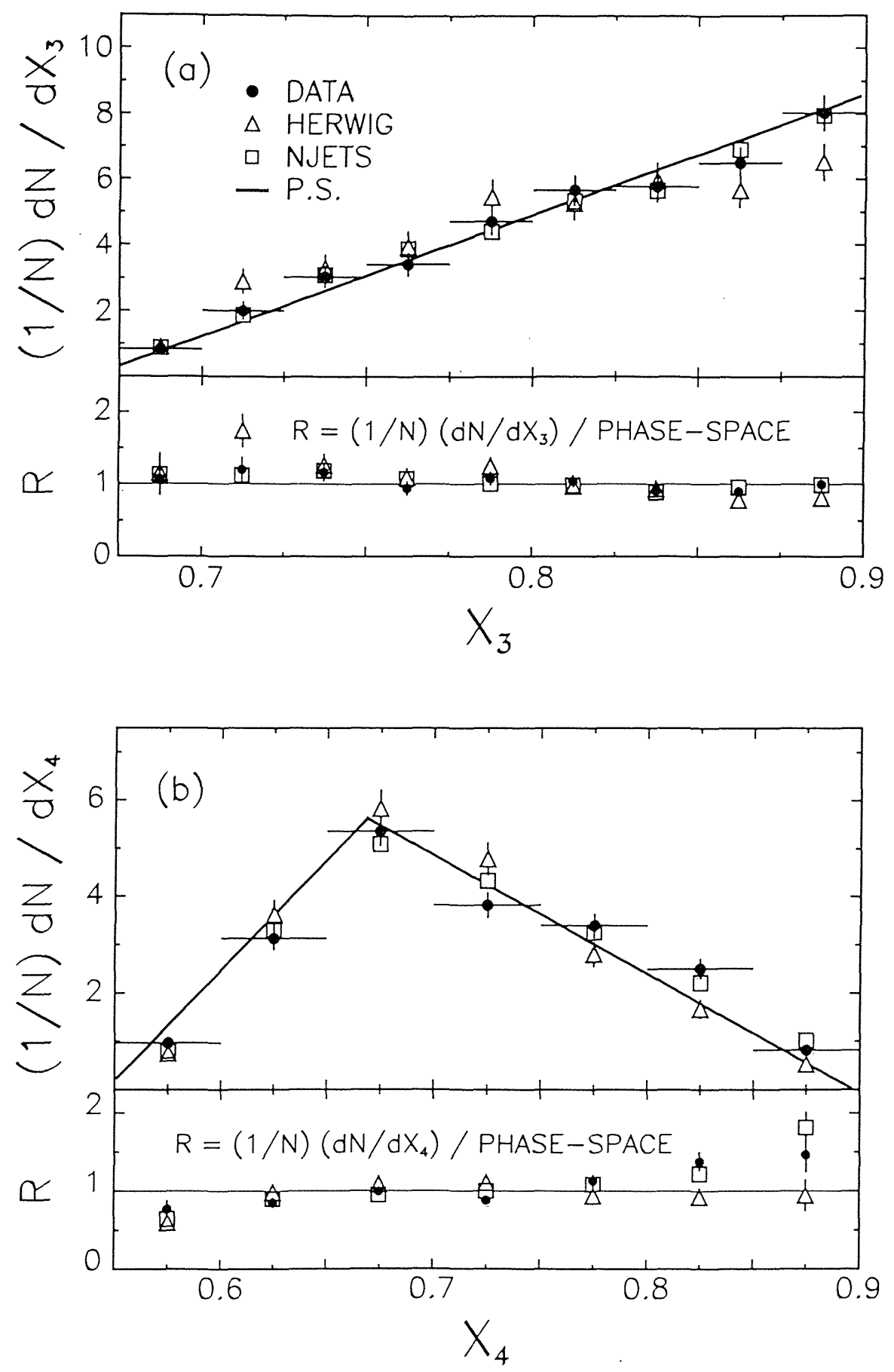

Figure 6.3: Inclusive three-jet Dalitz distributions for events that satisfy the requirements $m_{3 J}>600 \mathrm{GeV} / c^{2}, X_{3}<0.9$, and $\left|\cos \theta_{3}\right|<0.6$. Data (points) are compared with HERWIG predictions (triangles), NJETS predictions (squares), and phase-space model predictions (curves) for (a) $X_{3}$, and (b) $X_{4}$. 


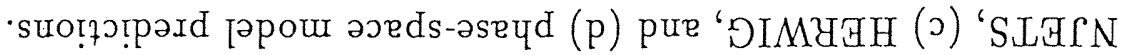

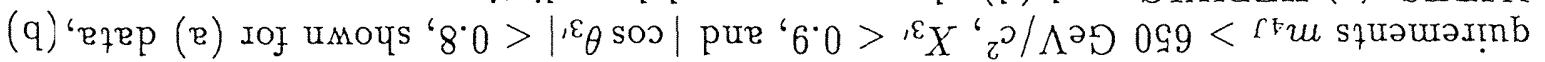

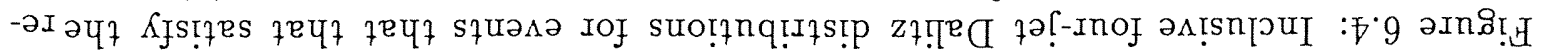

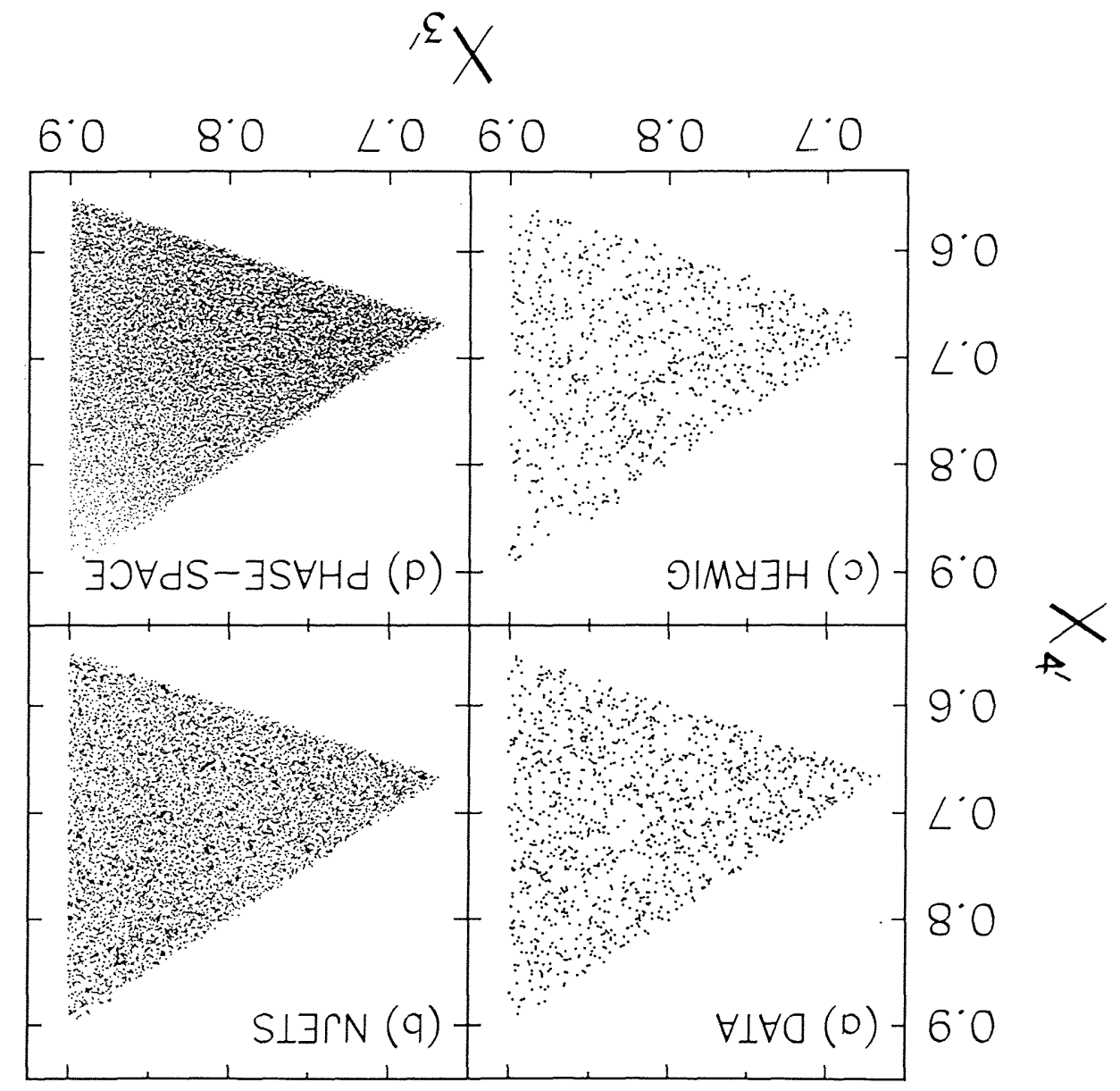



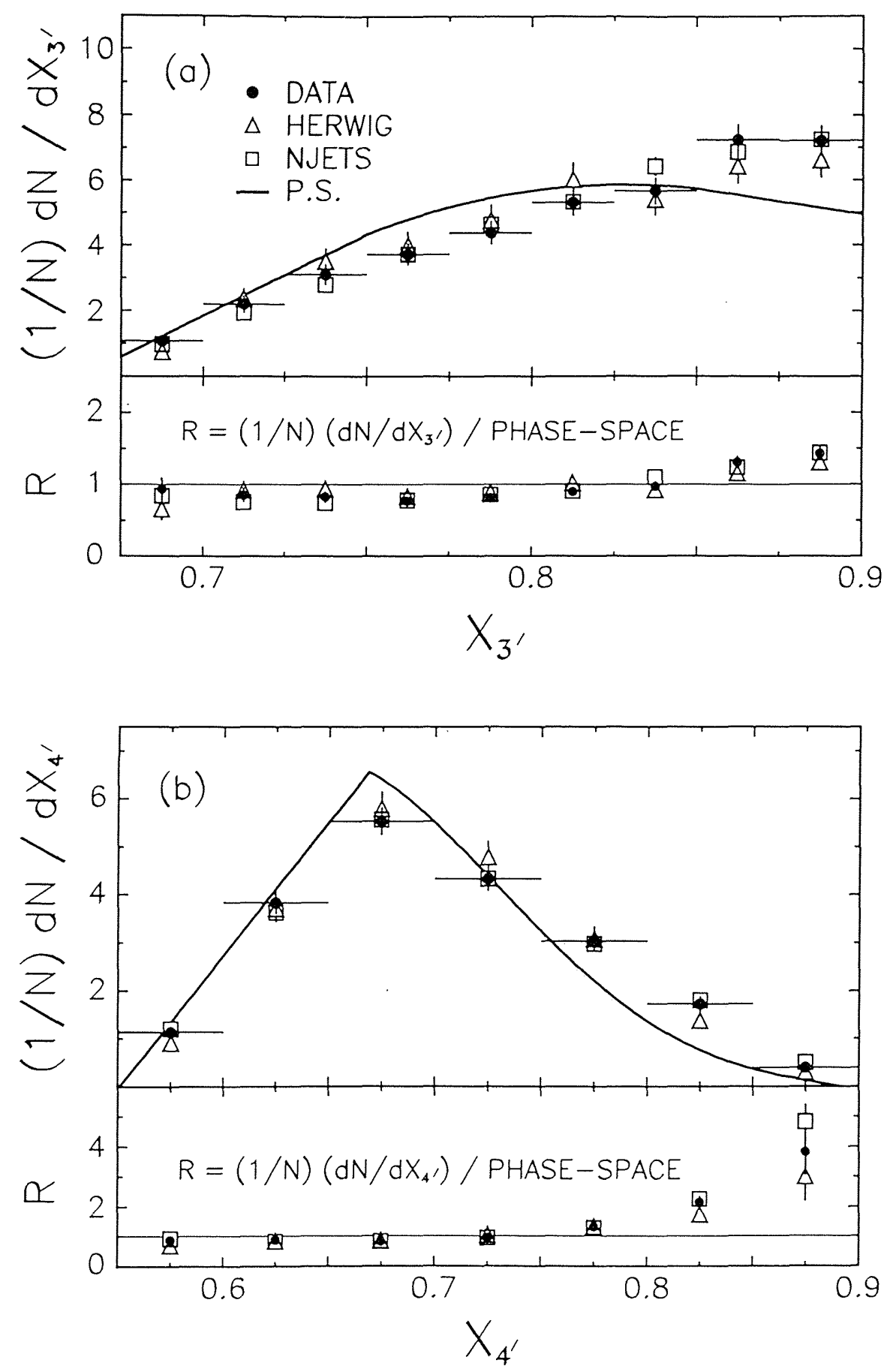

Figure 6.5: Dalitz distributions for inclusive four-jet topologies that satisfy the requirements $m_{4 J}>650 \mathrm{GeV} / c^{2}, X_{3^{\prime}}<0.9$, and $\left|\cos \theta_{3^{\prime}}\right|<0.8$. Data (points) are compared with HERWIG predictions (triangles), NJETS predictions (squares), and phase-space model predictions (curves) for (a) $X_{3^{\prime}}$, and (b) $X_{4^{\prime}}$. 


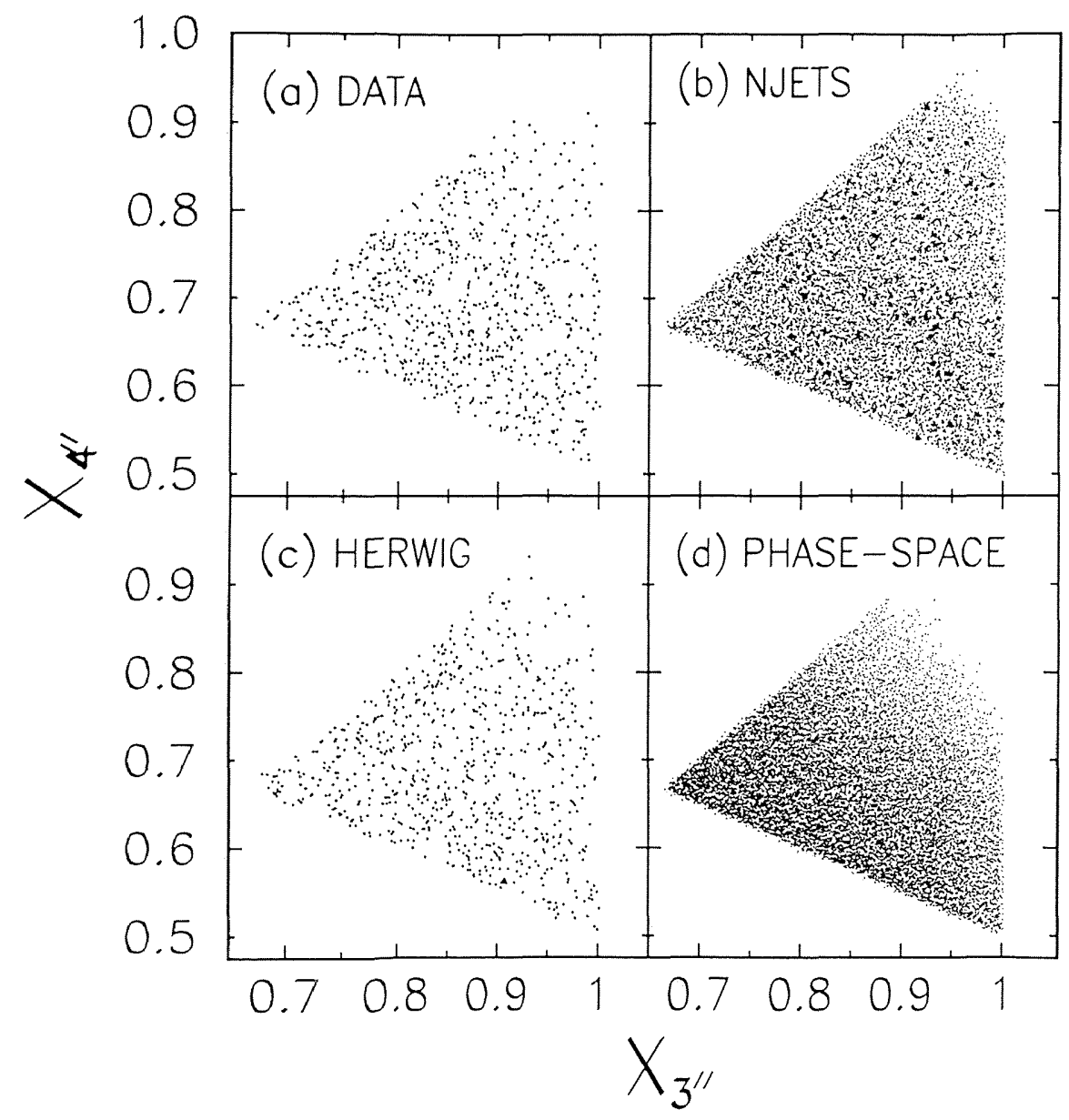

Figure 6.6: Inclusive five-jet Dalitz distributions for events that satisfy the requirement $m_{5 J}>750 \mathrm{GeV} / c^{2}$, shown for (a) data, (b) NJETS, (c) HERWIG, and (d) phase-space model predictions. 

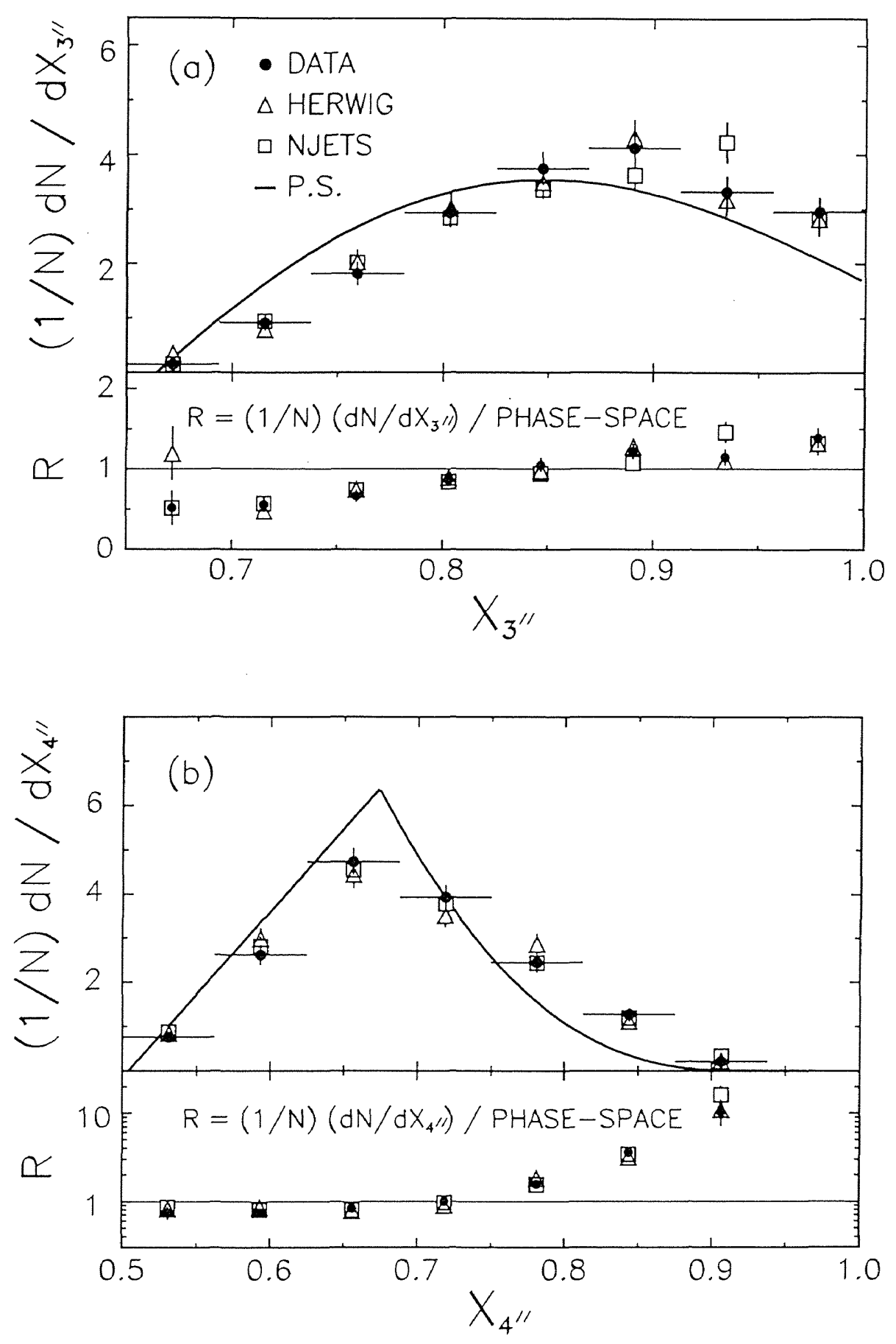

Figure 6.7: Dalitz distributions for inclusive five-jet topologies that satisfy the requirement $m_{5 J}>750 \mathrm{GeV} / c^{2}$. Data (points) are compared with HERWIG predictions (triangles), NJETS predictions (squares), and phase-space model predictions (curves) for (a) $X_{3^{\prime \prime}}$, and (b) $X_{4^{\prime \prime}}$. 


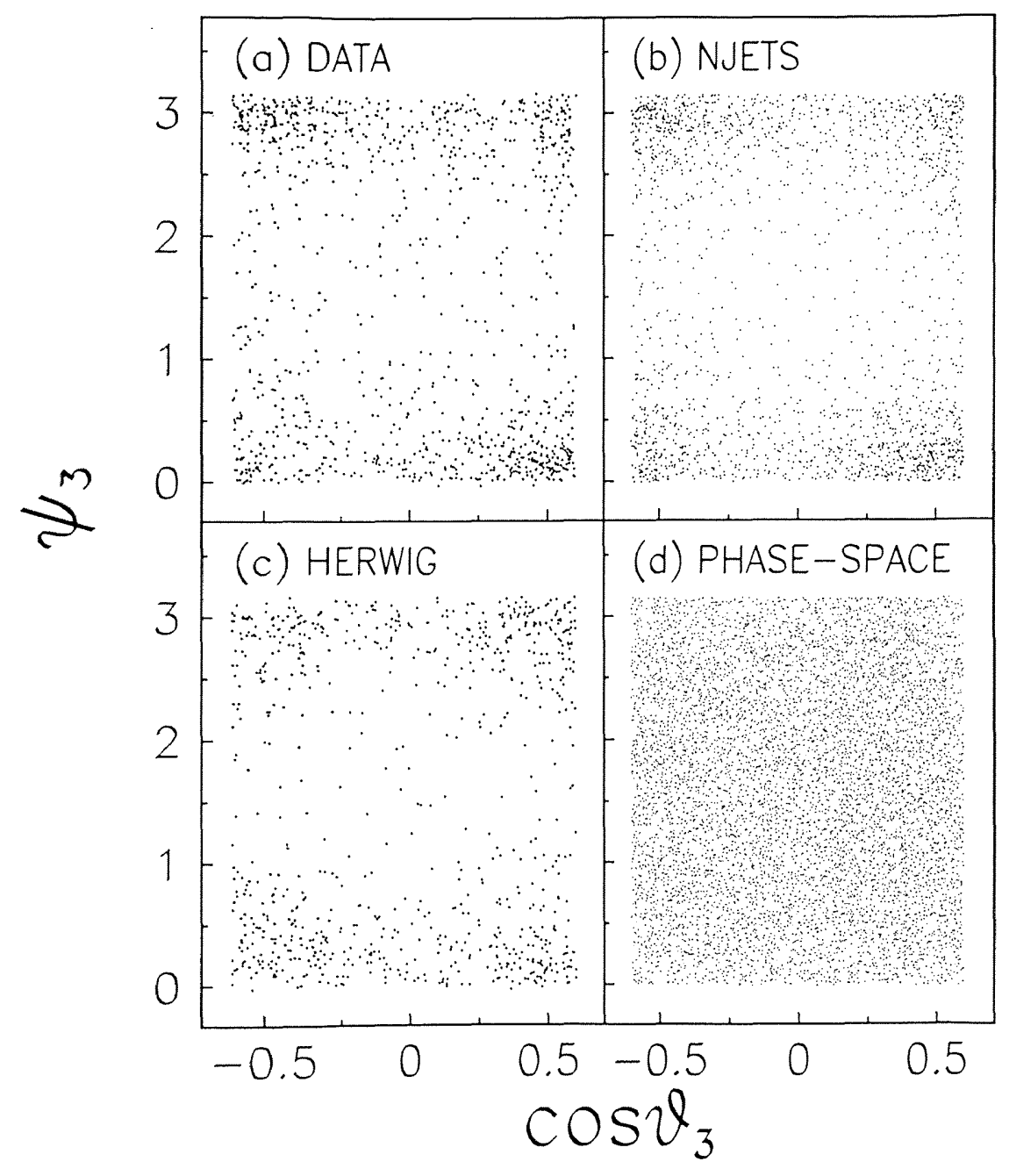

Figure 6.8: Inclusive three-jet angular distributions for events that satisfy the requirements $m_{3 J}>600 \mathrm{GeV} / c^{2}, X_{3}<0.9$, and $\left|\cos \theta_{3}\right|<0.6$. Event populations in the $\left(\cos \theta_{3}, \psi_{3}\right.$ )-plane are shown for (a) data, (b) NJETS, (c) HERWIG, and (d) phasespace model predictions. 

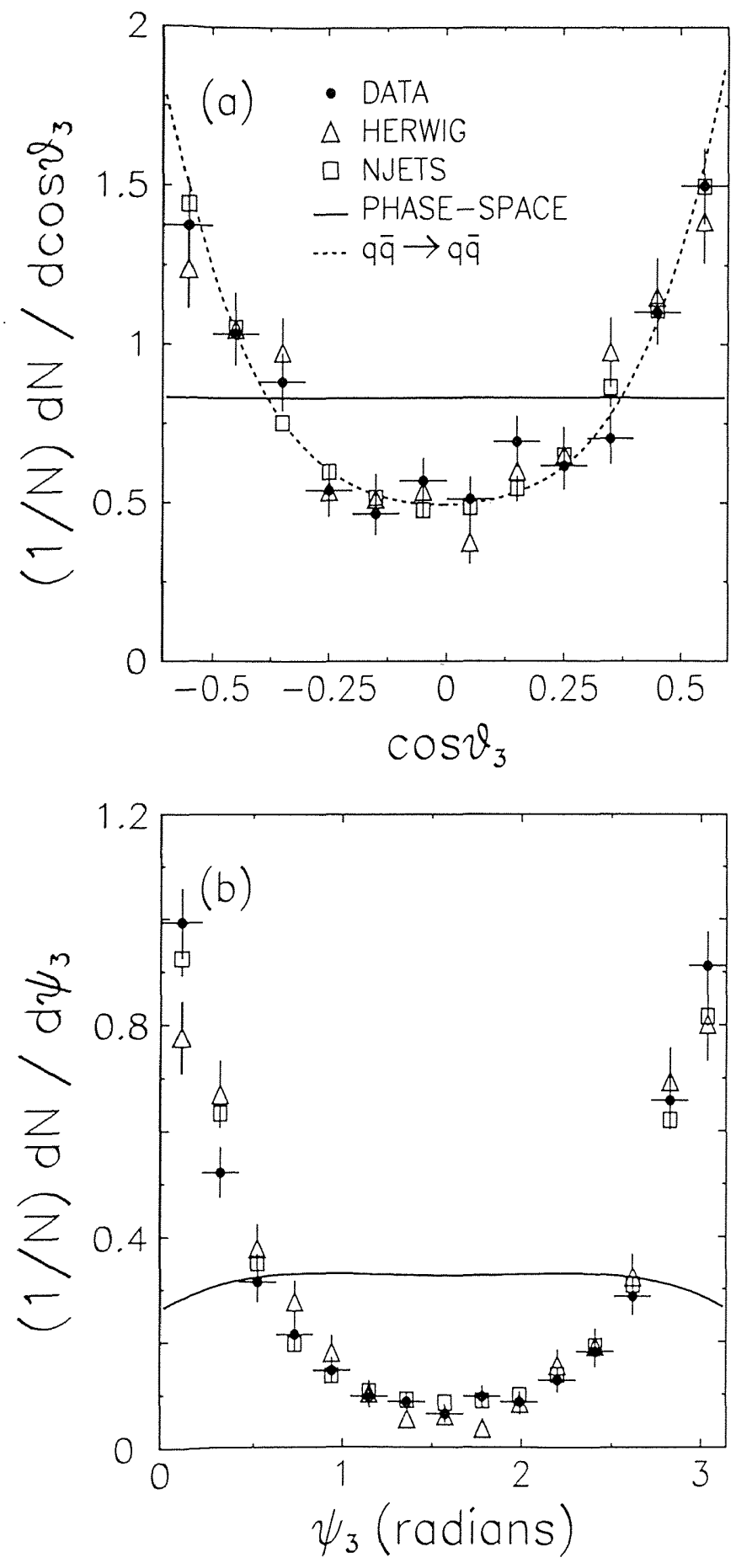

Figure 6.9: Inclusive three-jet angular distributions for events that satisfy the requirements $m_{3 J}>600 \mathrm{GeV} / c^{2}, X_{3}<0.9$, and $\left|\cos \theta_{3}\right|<0.6$. Data (points) are compared with HERWIG predictions (triangles), NJETS predictions (squares), and phase-space model predictions (curves) for (a) $\cos \theta_{3}$ and (b) $\psi_{3}$. The broken curve in the $\cos \theta_{3}$ figure is the LO QCD prediction for $q \bar{q} \rightarrow q \bar{q}$ scattering. 


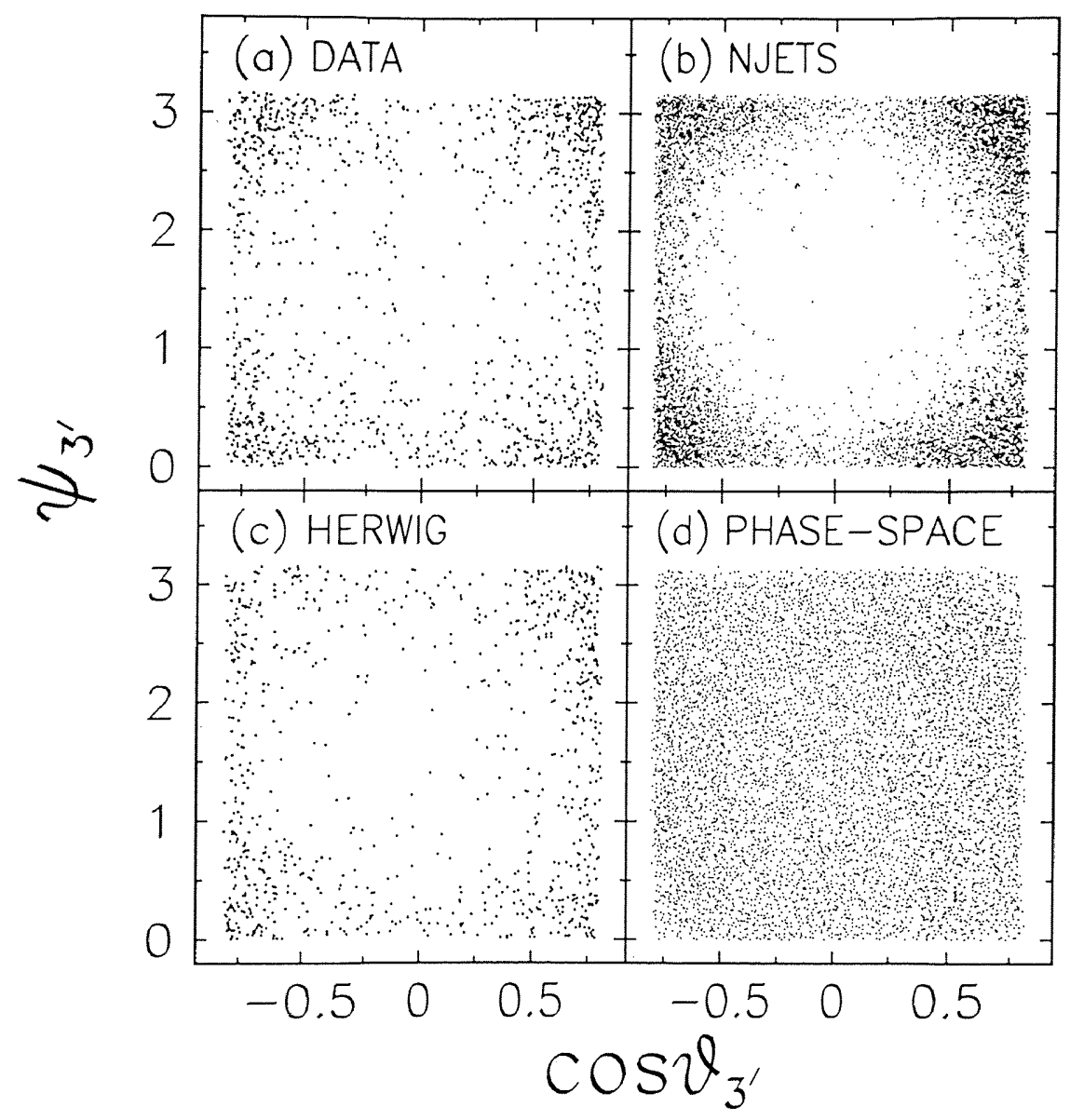

Figure 6.10: Inclusive four-jet angular distributions for events that satisfy the requirements $m_{4 J}>650 \mathrm{GeV} / c^{2}, X_{3^{\prime}}<0.9$, and $\left|\cos \theta_{3^{\prime}}\right|<0.8$. Event populations in the $\left(\cos \theta_{3^{\prime}}, \psi_{3^{\prime}}\right)$-plane are shown for (a) data, (b) NJETS, (c) HERWIG, and (d) phasespace model predictions. 

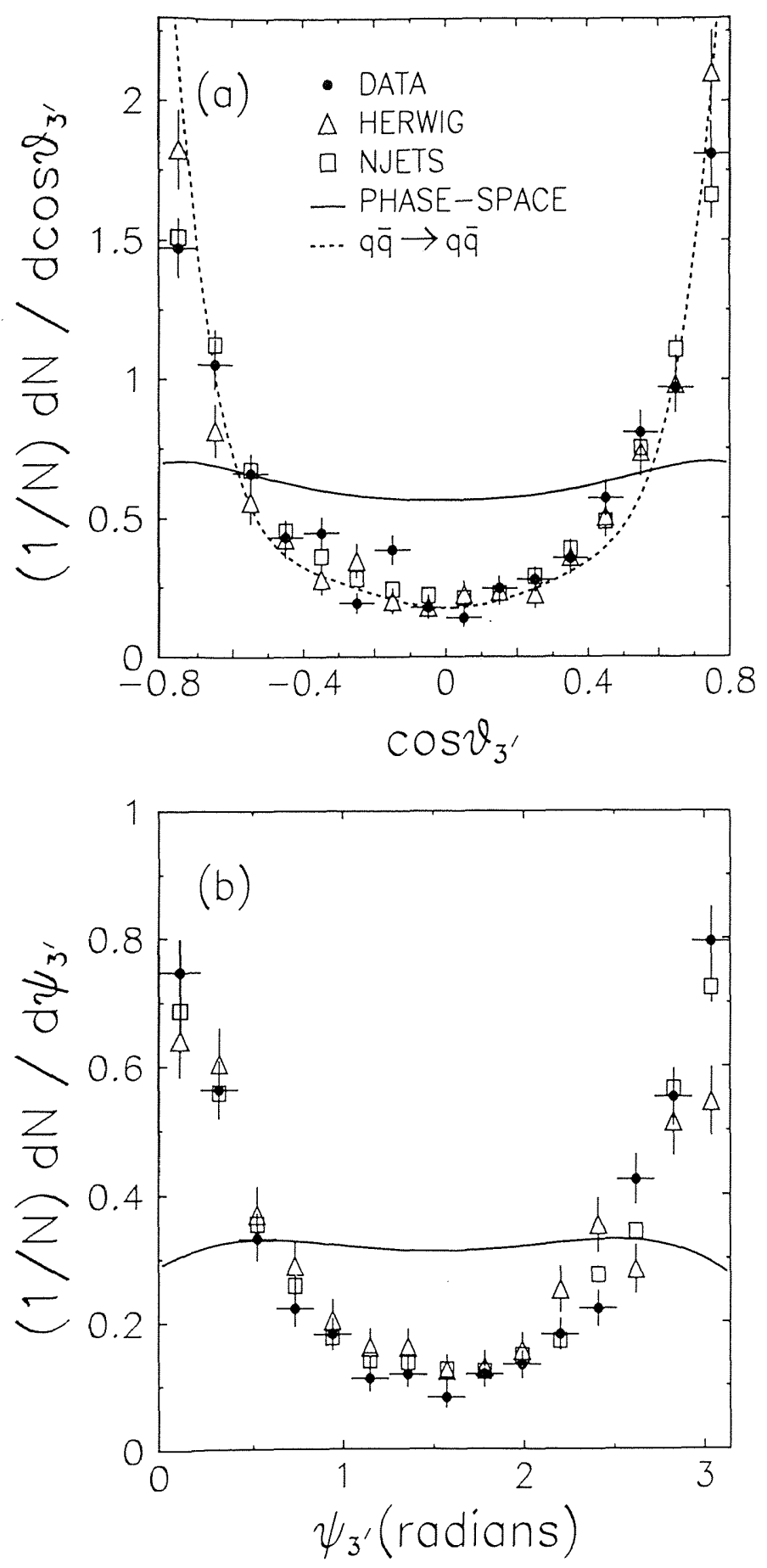

Figure 6.11: Inclusive four-jet angular distributions for events that satisfy the requirements $m_{4 J}>650 \mathrm{GeV} / c^{2}, X_{3^{\prime}}<0.9$, and $\left|\cos \theta_{3^{\prime}}\right|<0.8$. Data (points) are compared with HERWIG predictions (triangles), NJETS predictions (squares), and phase-space model predictions (curves) for (a) $\cos \theta_{3^{\prime}}$ and (b) $\psi_{3^{\prime}}$. The broken curve in the $\cos \theta_{3^{\prime}}$ figure is the LO QCD prediction for $q \bar{q} \rightarrow q \bar{q}$ scattering. 


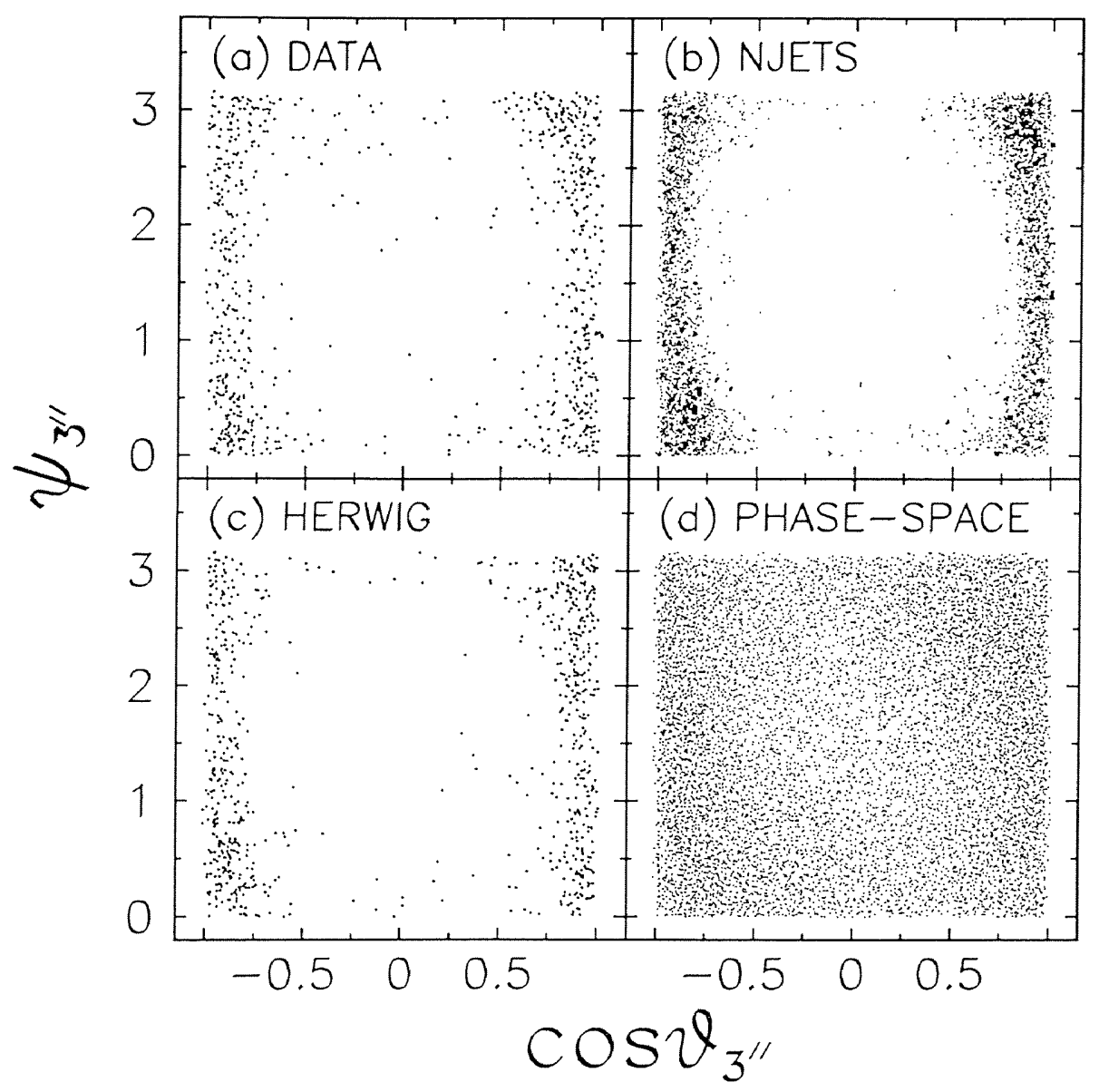

Figure 6.12: Inclusive five-jet angular distributions for events that satisfy the requirement $m_{5 J}>750 \mathrm{GeV} / c^{2}$. Event populations in the $\left(\cos \theta_{3^{\prime \prime}}, \psi_{3^{\prime \prime}}\right)$-plane are shown for (a) data, (b) NJETS, (c) HERWIG, and (d) phase-space model predictions. 

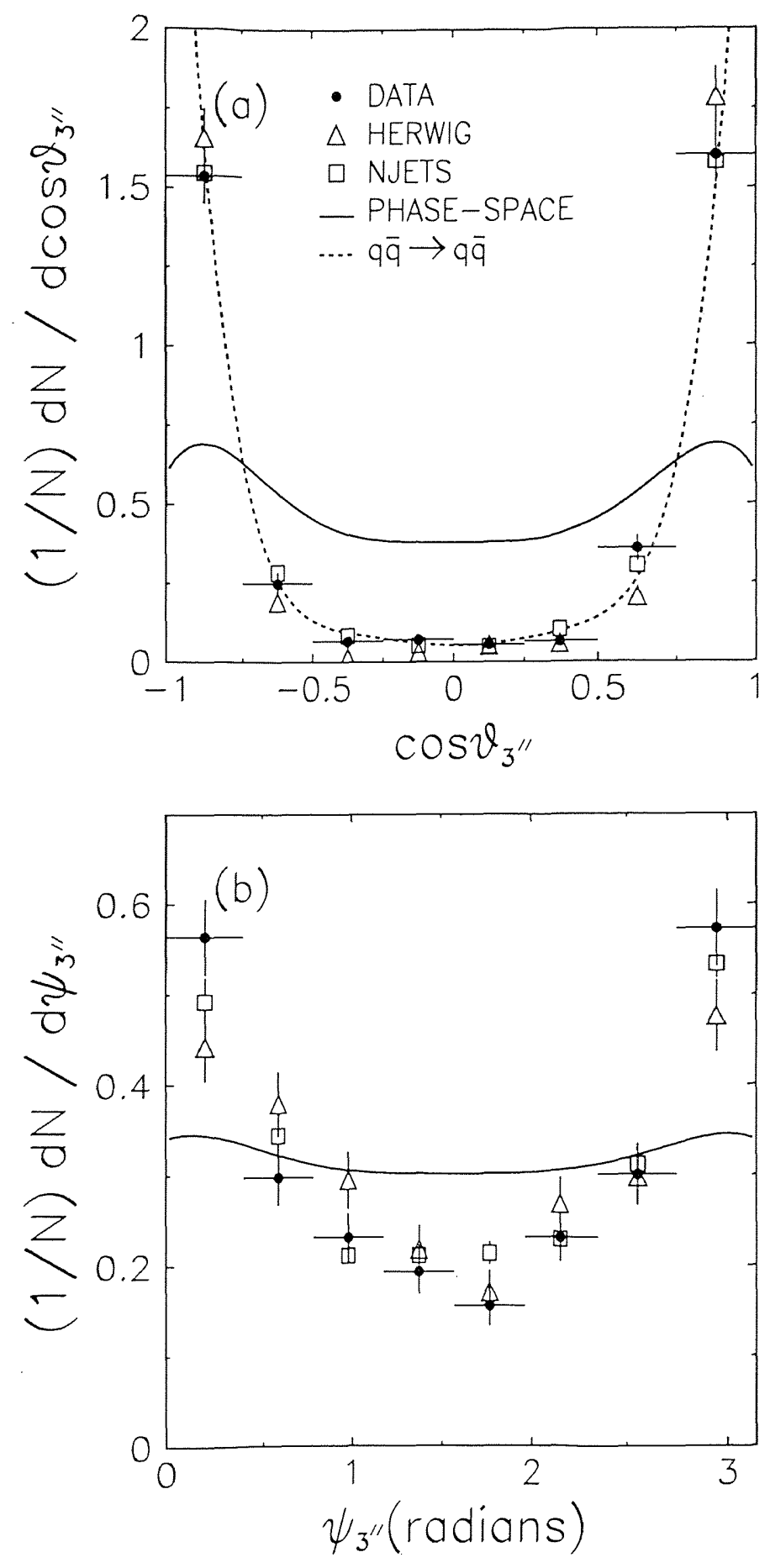

Figure 6.13: Inclusive five-jet angular distributions for events that satisfy the requirement $m_{5 J}>750 \mathrm{GeV} / c^{2}$. Data (points) are compared with HERWIG predictions (triangles), NJETS predictions (squares), and phase-space model predictions (curves) for (a) $\cos \theta_{3^{\prime \prime}}$ and (b) $\psi_{3^{\prime \prime}}$. The broken curve in the $\cos \theta_{3^{\prime \prime}}$ figure is the LO QCD prediction for $q \bar{q} \rightarrow q \bar{q}$ scattering. 


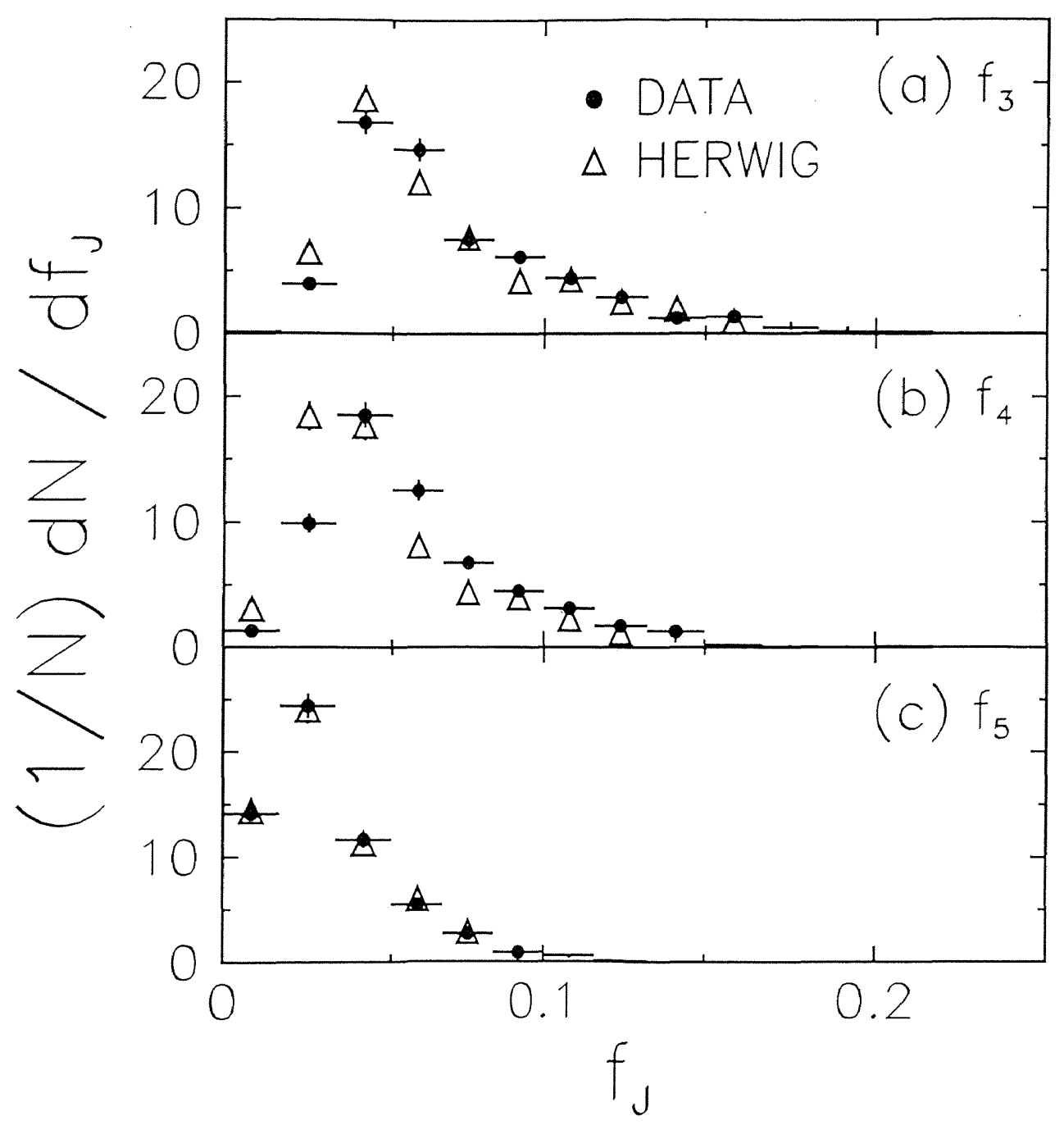

Figure 6.14: Single-jet mass fraction distributions for inclusive three-jet events. Data (points) compared with HERWIG predictions (triangles), shown for (a) the highest energy jet in the three-jet rest-frame, (b) the second-to-highest energy jet, and (c) the third-to-highest energy jet. 


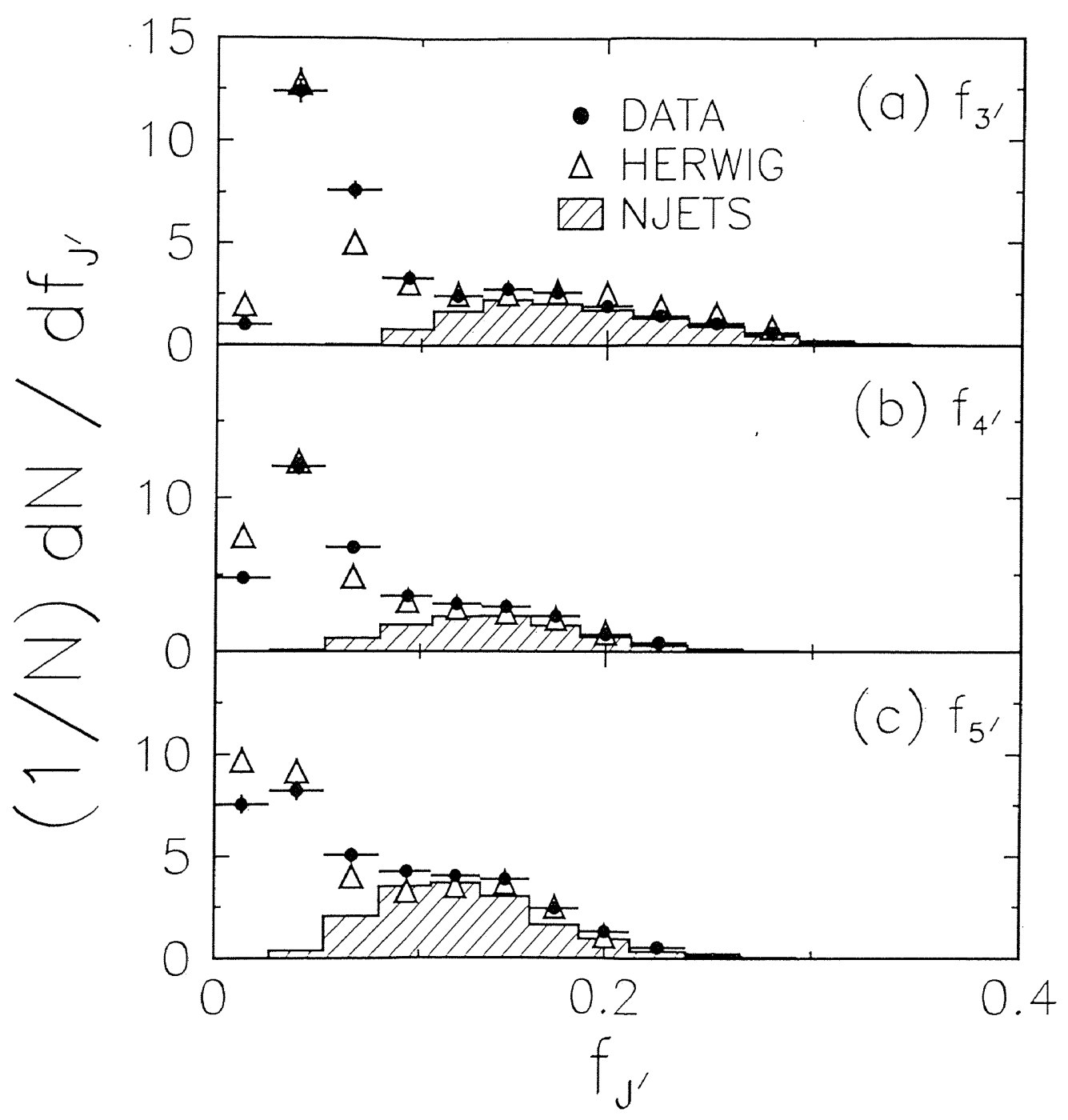

Figure 6.15: Single-body mass fraction distributions for inclusive four-jet events. Data (points) compared with HERWIG predictions (triangles), and NJETS predictions (histograms), shown for (a) the highest energy body in the three-body rest-frame, (b) the second-to-highest energy body, and (c) the third-to-highest energy body. 


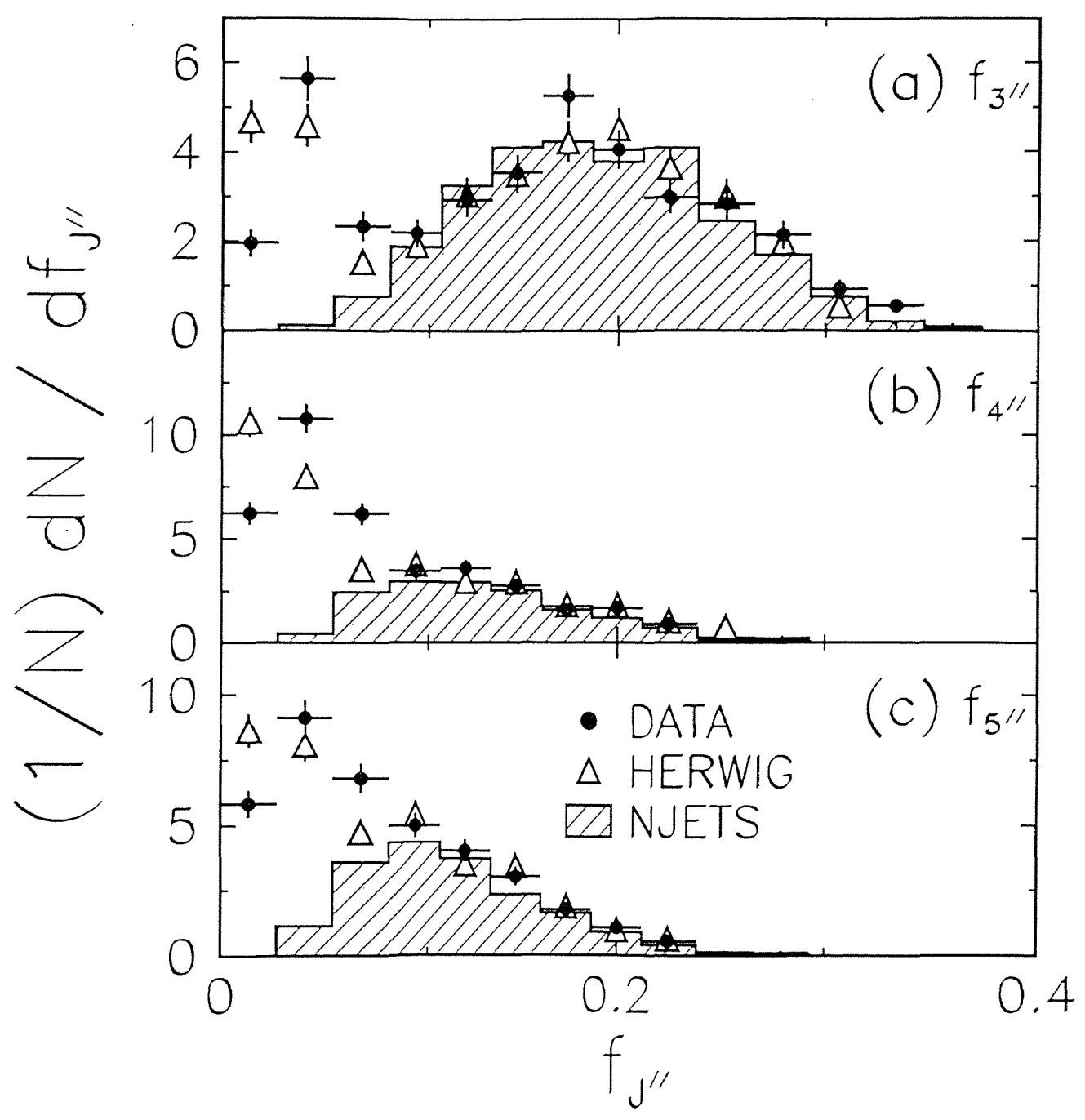

Figure 6.16: Single-body mass fraction distributions for inclusive five-jet events. Data (points) compared with HERWIG predictions (triangles), and NJETS predictions (histograms), shown for (a) the highest energy body in the three-body rest-frame, (b) the second-to-highest energy body, and (c) the third-to-highest energy body. 

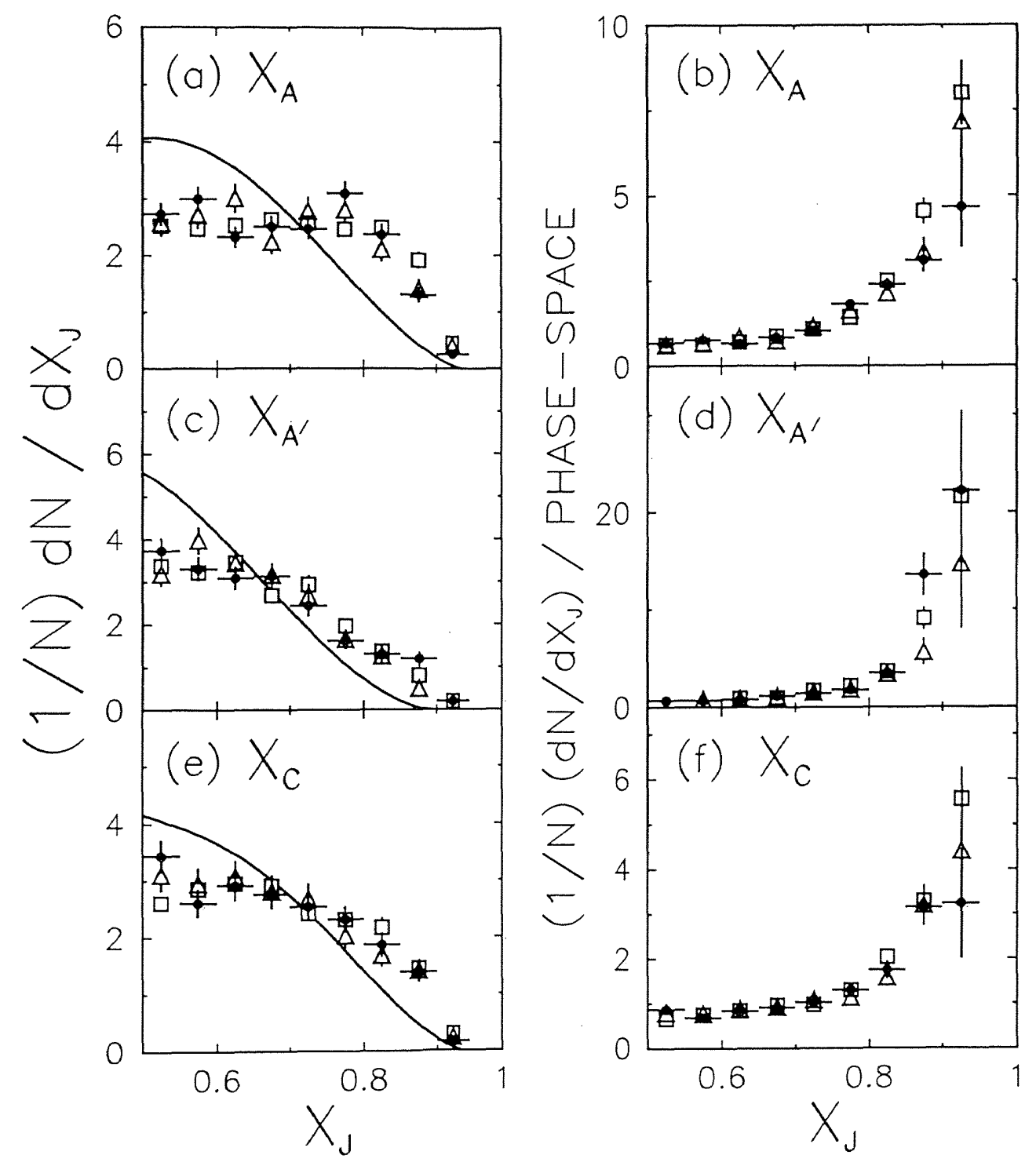

Figure 6.17: The two-body energy sharing distributions for inclusive four-jet and fivejet events. Data (points) are compared with HERWIG predictions (triangles), NJETS predictions (squares), and phase-space predictions (curves) for (a) $X_{A}$, (b) $X_{A}$ after dividing by the phase-space model predictions, (c) $X_{A^{\prime}}$, (d) $X_{A^{\prime}}$ after dividing by the phase-space model predictions, (e) $X_{C}$, and (f) $X_{C}$ after dividing by the phase-space model predictions. 

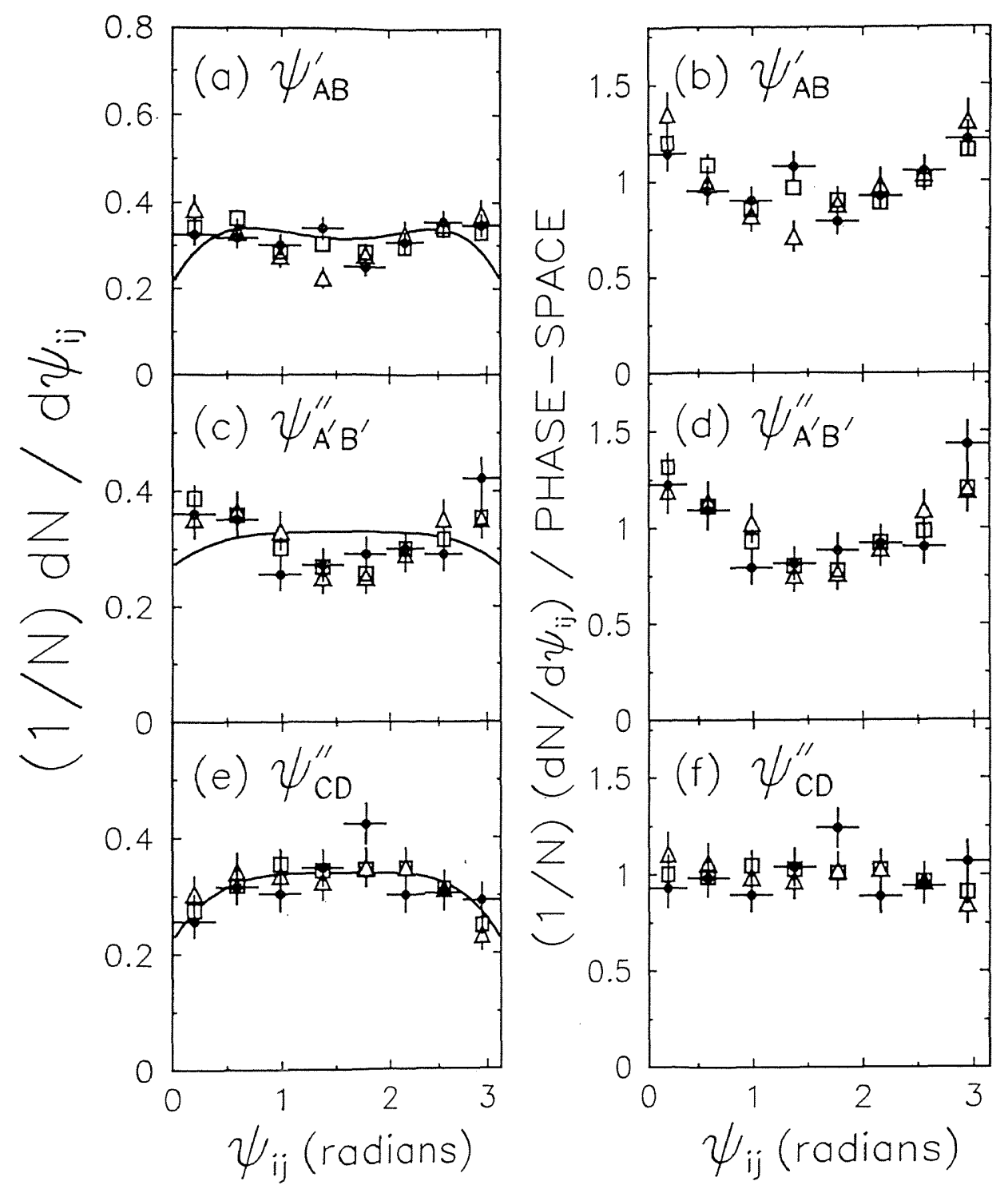

Figure 6.18: Two-body angular distributions for inclusive four-jet and five-jet events. Data (points) are compared with HERWIG predictions (triangles), NJETS predictions (squares), and phase-space predictions (curves) for (a) $\psi_{A B}^{\prime}$, (b) $\psi_{A B}^{\prime}$ after dividing by the phase-space model predictions, (c) $\psi_{A^{\prime} B^{\prime}}^{\prime \prime}$, (d) $\psi_{A^{\prime} B^{\prime}}^{\prime \prime}$ after dividing by the phasespace model predictions, (e) $\psi_{C D}^{\prime \prime}$, and (f) $\psi_{C D}^{\prime \prime}$ after dividing by the phase-space model predictions. 


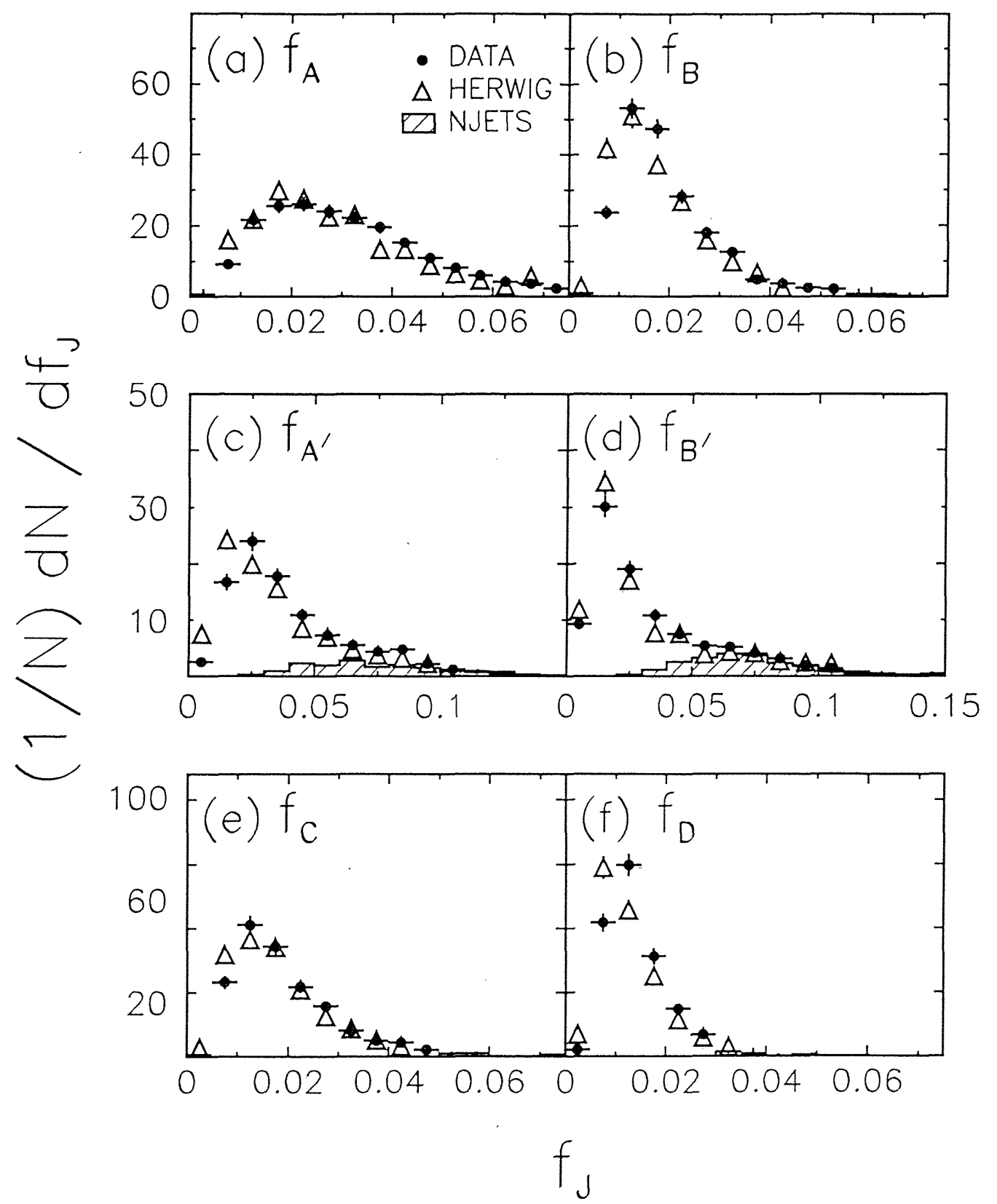

Figure 6.19: Single-body mass fraction distributions for two-body systems in inclusive four-jet and five-jet events. Data (points) are compared with HERWIG predictions (triangles), and NJETS predictions (histograms) for (a) $f_{A}$, (b) $f_{B}$, (c) $f_{A^{\prime}}$, (d) $f_{B^{\prime}}$, (e) $f_{C}$, and (f) $f_{D}$. 


\section{Chapter 7}

\section{Systematic Studies}

We have performed a number of systematic studies to check that our results are not subject to large theoretical uncertainties associated with choice of structure function, $Q^{2}$-scale, or effective minimum jet-jet separation. We have also checked that there are no large experimental uncertainties associated with contributions from multiple interactions at high luminosity or with the uncertainties on the jet energy scale.

\subsection{Theoretical Uncertainties}

We have compared the NJETS predictions of multijet events for structure function of KMRSD0, $\Delta R_{\min }=0.9$, and $Q^{2}$-scale of $\left\langle p_{T}\right\rangle^{2}$ with the observed data and the HERWIG predictions in this analysis. In order to check whether our multijet variables are sensitive to these parameter choices, we have generated NJETS Monte Carlo samples of three- and four-jet events for eight different sets of structure functions, $Q^{2}$-scales and $\triangle R_{\min }$ listed in Table 7.1. In Figures 7.1-7.6 NJETS predictions of the distributions of our three- and four-jet variables for these parameter choices (curves) are shown to be compared with the observed distributions (points). The $\chi^{2} \mathrm{~s}$, in which the statistical comparisons of the NJETS distributions to the observed distributions result, are summarized in Tables 7.2 and 7.3 for three-jet events and four-jet events respectively. We find that the NJETS predictions for our multijet variables are seen to be insensitive to these parameter choices. 


\begin{tabular}{c|lcc}
\hline \hline & Structure & & \\
Choice & Function & $Q^{2}$-scale & $\Delta R_{\min }$ \\
\hline 1 & CTEQ1M & $\left\langle p_{T}\right\rangle^{2}$ & 0.9 \\
2 & CTWQ1MS & $\left\langle p_{T}\right\rangle^{2}$ & 0.9 \\
3 & KMRSS0 & $\left\langle p_{T}\right\rangle^{2}$ & 0.9 \\
4 & KMRSD0 & $\left\langle p_{T}\right\rangle^{2}$ & 0.9 \\
5 & KMRSD- & $\left\langle p_{T}\right\rangle^{2}$ & 0.9 \\
6 & KMRSD0 & $\left\langle p_{T}\right\rangle^{2}$ & 0.8 \\
7 & KMRSD0 & $\left\langle p_{T}\right\rangle^{2}$ & 1.0 \\
8 & KMRSD0 & $m^{2}$ & 0.9 \\
\hline \hline
\end{tabular}

Table 7.1: Parameter choices used for the eight NJETS calculations.

\begin{tabular}{c|ccccccccc|c}
\hline \hline 3-jet & \multicolumn{7}{|c|}{$\chi^{2} /$ n.d.f. (NJETS-DATA) } & \\
\cline { 2 - 10 } variables & choice & 1 & 2 & 3 & 4 & 5 & 6 & 7 & 8 & n.d.f. \\
\hline$m_{3 J}$ & 1.10 & 0.73 & 0.85 & 1.27 & 1.32 & 1.18 & 1.31 & 1.43 & 6 \\
$X_{3}$ & 0.28 & 0.55 & 0.81 & 0.41 & 0.71 & 0.48 & 0.52 & 0.78 & 8 \\
$X_{4}$ & 1.19 & 1.45 & 1.28 & 1.42 & 1.59 & 0.91 & 1.03 & 0.79 & 6 \\
$\cos \theta_{3}$ & 1.03 & 1.10 & 0.93 & 0.95 & 1.01 & 0.73 & 1.06 & 0.91 & 11 \\
$\psi_{3}$ & 0.90 & 0.76 & 1.01 & 0.84 & 1.05 & 1.17 & 1.02 & 1.99 & 14 \\
\hline over all & 0.89 & 0.90 & 0.97 & 0.93 & 1.08 & 0.91 & 0.98 & 1.28 & 45 \\
\hline \hline
\end{tabular}

Table 7.2: Statistical comparison of agreement between observed 3-jet distributions and distributions predicted by NJETS for eight parameter choices. The $\chi^{2}$ s per degree of freedom are listed.

\begin{tabular}{|c|c|c|c|c|c|c|c|c|c|c|}
\hline \multirow{2}{*}{$\begin{array}{c}4 \text {-jet } \\
\text { variables }\end{array}$} & \multicolumn{9}{|c|}{$\chi^{2} /$ n.d.f. (NJETS-DATA) } & \multirow[b]{2}{*}{ n.d.f. } \\
\hline & choice & 1 & 2 & 3 & 4 & 5 & 6 & 7 & 8 & \\
\hline$m_{4, J}$ & & 0.15 & 0.69 & 0.24 & 0.66 & 0.19 & 0.57 & 0.28 & 0.60 & 6 \\
\hline$X_{3^{\prime}}$ & & 0.85 & 0.42 & 0.78 & 0.66 & 0.79 & 0.73 & 0.68 & 0.62 & 8 \\
\hline$X_{4^{\prime}}$ & & 1.19 & 0.62 & 0.67 & 0.40 & 0.86 & 0.85 & 0.58 & 0.81 & 6 \\
\hline $\cos \theta_{3^{\prime}}$ & & 2.07 & 1.72 & 2.36 & 1.70 & 2.35 & 2.02 & 1.63 & 2.33 & 15 \\
\hline$\psi_{3^{\prime}}$ & & 1.09 & 0.76 & 0.95 & 1.36 & 0.93 & 0.90 & 1.33 & 2.03 & 14 \\
\hline$X_{A}$ & & 4.45 & 3.25 & 3.87 & 3.80 & 4.14 & 3.29 & 2.59 & 3.60 & 8 \\
\hline$\psi_{A B}^{\prime}$ & & 1.31 & 0.93 & 1.12 & 1.01 & 1.08 & 1.39 & 0.75 & 1.07 & 7 \\
\hline over all & & 1.65 & 1.25 & 1.55 & 1.47 & 1.59 & 1.46 & 1.24 & 1.76 & 64 \\
\hline
\end{tabular}

Table 7.3: Statistical comparison of agreement between observed 4-jet distributions and distributions predicted by NJETS for eight parameter choices. The $\chi^{2}$ s per degree of freedom are listed. 


\subsection{Influence from Multiple Interactions}

To understand whether there is a significant distortion of our multijet distributions due to contributions from multiple interactions, we have separated our multijet sample into three subsamples containing events detected at the highest luminosities $\left(\mathcal{L}>8 \times 10^{30}\right.$ $\left.\mathrm{cm}^{-2} \mathrm{~s}^{-1}\right)$, at intermediate luminosities $\left(4<\mathcal{L}<8 \times 10^{30} \mathrm{~cm}^{-2} \mathrm{~s}^{-1}\right)$, and at low luminosities $\left(\mathcal{L}<4 \times 10^{30} \mathrm{~cm}^{-2} \mathrm{~s}^{-1}\right.$ ) (see Figure 7.7). The number of interactions $N_{\text {int }}$ per beam crossing expected for each luminosity range is:

$$
\begin{array}{lll}
N_{\text {int }} \sim 0.4 & \text { for } & \mathcal{L}=2 \times 10^{30} \mathrm{~cm}^{-2} \mathrm{~s}^{-1} \\
N_{\text {int }} \sim 1.1 & \text { for } & \mathcal{L}=6 \times 10^{30} \mathrm{~cm}^{-2} \mathrm{~s}^{-1} \\
N_{\text {int }} \sim 1.8 & \text { for } & \mathcal{L}=1 \times 10^{31} \mathrm{~cm}^{-2} \mathrm{~s}^{-1}
\end{array}
$$

Then the observed distributions in the three luminosity ranges are compared with each other in Figures 7.8-7.10, 7.11-7.14, and 7.15-7.19 for three-, four-, and five-jet variables respectively. In all the figures points, histograms, and squares correspond to events measured at the highest luminosities, intermediate luminosities, and low luminosities. We have performed the statistical comparison by calculating $\chi^{2}$ s between the observed data and the corresponding NJETS predictions of our multijet variables in each of luminosity range as shown in Tables 7.4, 7.5, 7.6 for three-, four-, and five-jet events. No luminosity dependence can be seen in these results.

\begin{tabular}{c|ccc|c}
\hline \hline \multirow{2}{*}{$\begin{array}{c}\text { 3-jet } \\
\text { variables }\end{array}$} & \multicolumn{3}{|c|}{$\chi^{2} /$ n.d.f. } & \\
\cline { 2 - 4 } & low & mid & high & n.d.f. \\
\hline$m_{3 J}$ & 1.38 & 1.07 & 0.33 & 6 \\
$X_{3}$ & 0.59 & 1.16 & 0.50 & 8 \\
$X_{4}$ & 0.57 & 0.88 & 0.76 & 6 \\
$\cos \theta_{3}$ & 1.23 & 0.95 & 0.73 & 11 \\
$\psi_{3}$ & 1.73 & 1.18 & 0.83 & 14 \\
\hline over all & 1.20 & 1.06 & 0.67 & 45 \\
\hline \hline
\end{tabular}

Table 7.4: Statistical comparison of agreement between NJETS predictions and observed distributions of 3 -jet events in three different luminosity ranges. low: $\mathcal{L}<$ $4 \times 10^{30} \mathrm{~cm}^{-2} \mathrm{~s}^{-1}$, mid: $4<\mathcal{L}<8 \times 10^{30} \mathrm{~cm}^{-2} \mathrm{~s}^{-1}$, and high: $\mathcal{L}>8 \times 10^{30} \mathrm{~cm}^{-2} \mathrm{~s}^{-1}$ respectively. The $\chi^{2}$ s per degree of freedom are listed. 


\begin{tabular}{c|ccc|c}
\hline \hline \multirow{2}{*}{$\begin{array}{c}\text { 4-jet } \\
\text { variables }\end{array}$} & \multicolumn{3}{|c|}{$\chi^{2} /$ n.d.f. } & \\
\cline { 2 - 4 } & low & mid & high & n.d.f. \\
\hline$m_{4 J}$ & 1.52 & 1.19 & 0.35 & 6 \\
$X_{3^{\prime}}$ & 1.34 & 1.61 & 0.75 & 8 \\
$X_{4^{\prime}}$ & 0.43 & 0.19 & 1.07 & 6 \\
$\cos \theta_{3^{\prime}}$ & 0.80 & 1.07 & 1.39 & 15 \\
$\psi_{3^{\prime}}$ & 1.99 & 0.53 & 1.58 & 14 \\
$\psi_{A B}^{\prime}$ & 0.99 & 1.10 & 1.41 & 7 \\
$X_{A}$ & 2.40 & 2.53 & 3.09 & 8 \\
\hline over all & 1.38 & 1.13 & 1.44 & 64 \\
\hline \hline
\end{tabular}

Table 7.5: Statistical comparison of agreement between NJETS predictions and observed distributions of 4 -jet events in three different luminosity ranges. low: $\mathcal{L}<$ $4 \times 10^{30} \mathrm{~cm}^{-2} \mathrm{~s}^{-1}$, mid: $4<\mathcal{L}<8 \times 10^{30} \mathrm{~cm}^{-2} \mathrm{~s}^{-1}$, and high: $\mathcal{L}>8 \times 10^{30} \mathrm{~cm}^{-2} \mathrm{~s}^{-1}$ respectively. The $\chi^{2}$ s per degree of freedom are listed.

vspace $5 \mathrm{~mm}$

\begin{tabular}{c|ccc|c}
\hline \hline \multirow{2}{*}{$\begin{array}{c}5 \text {-jet } \\
\text { variables }\end{array}$} & \multicolumn{3}{|c|}{$\chi^{2} /$ n.d.f. } & \multirow{2}{*}{} \\
\cline { 2 - 4 } & low & mid & high & n.d.f. \\
\hline$m_{5 J}$ & 1.53 & 1.01 & 0.42 & 8 \\
$X_{3^{\prime \prime}}$ & 0.16 & 1.20 & 1.25 & 7 \\
$X_{4^{\prime \prime}}$ & 2.17 & 0.50 & 0.08 & 6 \\
$\cos \theta_{3^{\prime \prime}}$ & 0.67 & 1.93 & 1.19 & 7 \\
$\psi_{3^{\prime \prime}}$ & 0.50 & 1.10 & 1.59 & 7 \\
$\psi_{A^{\prime} B^{\prime}}^{\prime \prime}$ & 1.79 & 0.60 & 0.90 & 7 \\
$\psi_{C D}^{\prime \prime}$ & 0.42 & 0.53 & 1.26 & 7 \\
$X_{A^{\prime}}$ & 0.94 & 2.10 & 0.68 & 7 \\
$X_{C}$ & 0.69 & 0.66 & 1.60 & 7 \\
\hline over all & 0.97 & 1.08 & 1.00 & 63 \\
\hline \hline
\end{tabular}

Table 7.6: Statistical comparison of agreement between NJETS predictions and observed distributions of 5 -jet events in three different luminosity ranges. low: $\mathcal{L}<$ $4 \times 10^{30} \mathrm{~cm}^{-2} \mathrm{~s}^{-1}$, mid: $4<\mathcal{L}<8 \times 10^{30} \mathrm{~cm}^{-2} \mathrm{~s}^{-1}$, and high: $\mathcal{L}>8 \times 10^{30} \mathrm{~cm}^{-2} \mathrm{~s}^{-1}$ respectively. The $\chi^{2}$ 's per degree of freedom are listed. 


\subsection{Jet Energy Scale}

Finally, to understand the uncertainties associated with the uncertainties on the jet energy scale we have increased (decreased) all measured jet energies by $+7 \%(-7 \%)$ and remade the multijet distributions. The resulting distributions are compared with the distributions using the nominal energy scale in Figures 7.20-7.22, 7.23-7.26, and 7.27-7.31 for three-, four-, and five-jet variables respectively. In all the figures points, histograms, and squares correspond to events with energies scaled by $+7 \%, 0 \%$, and $-7 \%$. In Tables 7.7-7.9 we have summarized the resulting $\chi^{2}$ s. We see that the agreements of observed multijet mass distributions with the NJETS predictions get worse as jet energies shifted by $+7 \%$ for all of three-, four-, and five-jet events. The multijet masses are the only dimensional variables, hence they are expected to be more sensitive to experimental uncertainties associated with the uncertainties on the jet energy scale than the other variables. Except for the multijet mass distributions we see that the uncertainty on the measured distributions associated with the uncertainty on the jet energy scale is less than the statistical uncertainties.

\begin{tabular}{c|ccc|c}
\hline \hline \multirow{2}{*}{$\begin{array}{c}3 \text {-jet } \\
\text { variables }\end{array}$} & \multicolumn{3}{|c|}{$\chi^{2} /$ n.d.f. } & \\
\cline { 2 - 4 } & $-7 \%$ & nominal & $+7 \%$ & n.d.f. \\
\hline$m_{3 \mathrm{~J}}$ & 0.41 & 1.27 & 4.51 & 6 \\
$X_{3}$ & 1.34 & 0.41 & 0.95 & 8 \\
$X_{4}$ & 0.64 & 1.42 & 1.17 & 6 \\
$\cos \theta_{3}$ & 1.46 & 0.95 & 1.44 & 11 \\
$\psi_{3}$ & 0.62 & 0.84 & 0.61 & 14 \\
\hline over all & 0.93 & 0.93 & 1.47 & 45 \\
\hline \hline
\end{tabular}

Table 7.7: Statistical comparison of agreement between NJETS predictions and observed distributions of 3 -jet events with energies shifted by $-7 \%, 0 \%$, and $+7 \%$. The $\chi^{2}$ s per degree of freedom are listed. 


\begin{tabular}{c|ccc|c}
\hline \hline 4-jet & \multicolumn{3}{|c|}{$\chi^{2} /$ n.d.f. } & \\
\cline { 2 - 4 } variables & $-7 \%$ & nominal & $+7 \%$ & n.d.f. \\
\hline$m_{4 J}$ & 0.36 & 0.66 & 4.85 & 6 \\
$X_{3^{\prime}}$ & 1.48 & 0.66 & 0.41 & 8 \\
$X_{4^{\prime}}$ & 1.03 & 0.40 & 0.72 & 6 \\
$\cos \theta_{3^{\prime}}$ & 0.71 & 1.70 & 0.85 & 15 \\
$\psi_{3^{\prime}}$ & 1.07 & 1.36 & 1.74 & 14 \\
$\psi_{A B}^{\prime}$ & 1.43 & 1.01 & 0.63 & 7 \\
$X_{A}$ & 2.52 & 3.80 & 3.23 & 8 \\
\hline over all & 1.19 & 1.47 & 1.63 & 64 \\
\hline \hline
\end{tabular}

Table 7.8: Statistical comparison of agreement between NJETS predictions and observed distributions of 4 -jet events with energies shifted by $-7 \%, 0 \%$, and $+7 \%$. The $\chi^{2}$ s per degree of freedom are listed.

\begin{tabular}{c|ccc|c}
\hline \multirow{2}{*}{$\begin{array}{c}5 \text {-jet } \\
\text { Variables }\end{array}$} & \multicolumn{3}{|c|}{$\chi^{2} /$ n.d.f. } & \\
\cline { 2 - 4 } & $-7 \%$ & nominal & $+7 \%$ & n.d.f. \\
\hline$m_{5 \mathrm{~J}}$ & 1.42 & 0.52 & 1.81 & 8 \\
$X_{3^{\prime \prime}}$ & 2.03 & 1.08 & 1.75 & 7 \\
$X_{4^{\prime \prime}}$ & 0.89 & 0.42 & 0.47 & 6 \\
$\cos \theta_{3^{\prime \prime}}$ & 2.88 & 1.12 & 0.57 & 7 \\
$\psi_{3^{\prime \prime}}$ & 0.79 & 1.49 & 2.09 & 7 \\
$\psi_{A^{\prime} B^{\prime}}$ & 1.12 & 0.95 & 1.60 & 7 \\
$\psi_{C D}^{\prime \prime}$ & 1.86 & 1.16 & 0.59 & 7 \\
$X_{A^{\prime}}$ & 2.38 & 1.76 & 1.84 & 7 \\
$X_{C}$ & 1.63 & 1.47 & 2.36 & 7 \\
\hline over all & 1.68 & 1.11 & 1.47 & 63 \\
\hline \hline
\end{tabular}

Table 7.9: Statistical comparison of agreement between NJETS predictions and observed distributions of 5 -jet events with energies shifted by $-7 \%, 0 \%$, and $+7 \%$. The $\chi^{2}$ s per degree of freedom are listed. 


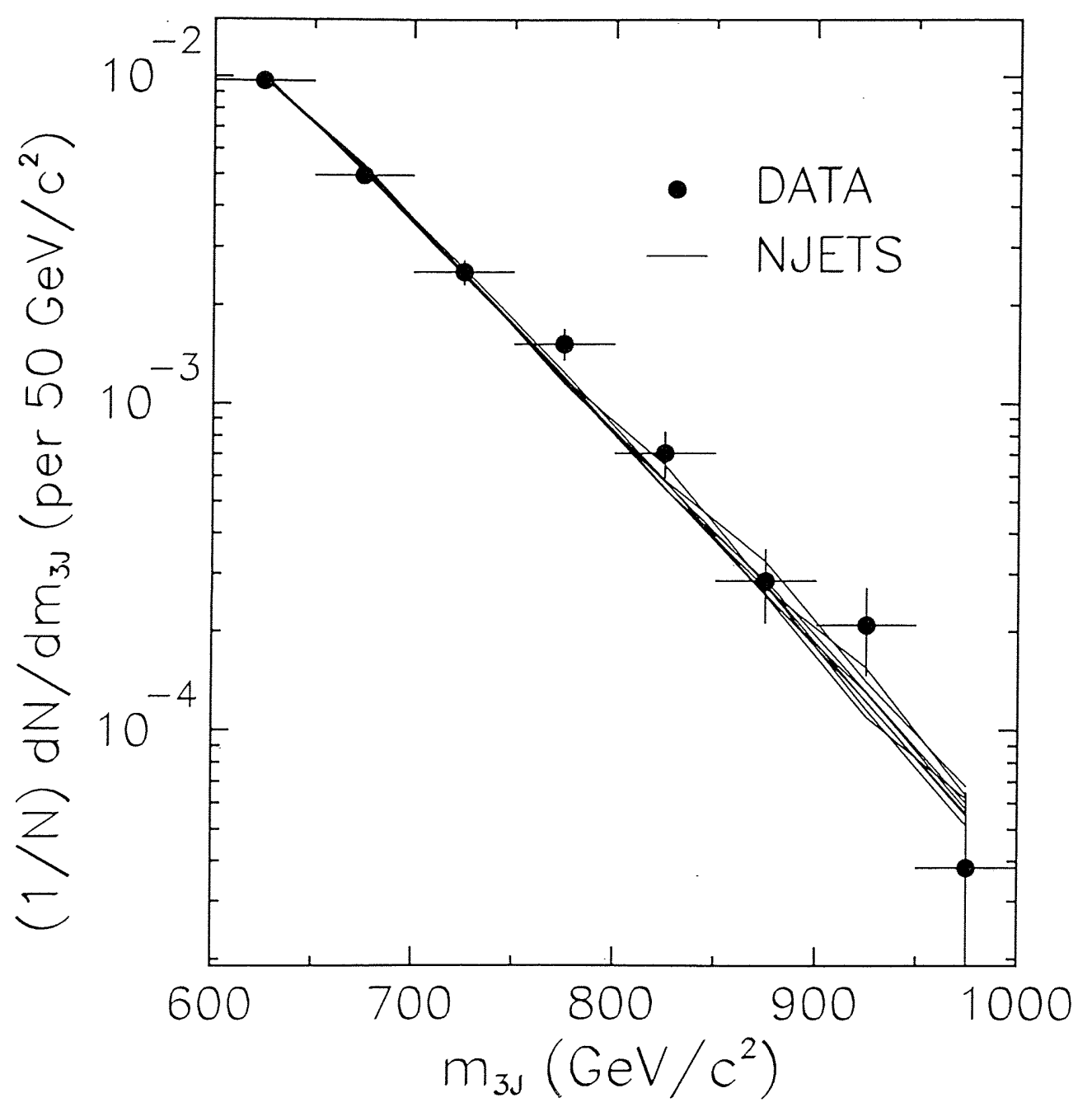

Figure 7.1: The NJETS predictions of the leading 3-jet mass distribution for various choices of structure functions, $Q^{2}$-scales, and $\Delta R_{\min }$. Eight curves in the figure correspond to the eight choices listed in Table 7.1. The histogram is the measured $m_{3 J}$ distribution. 

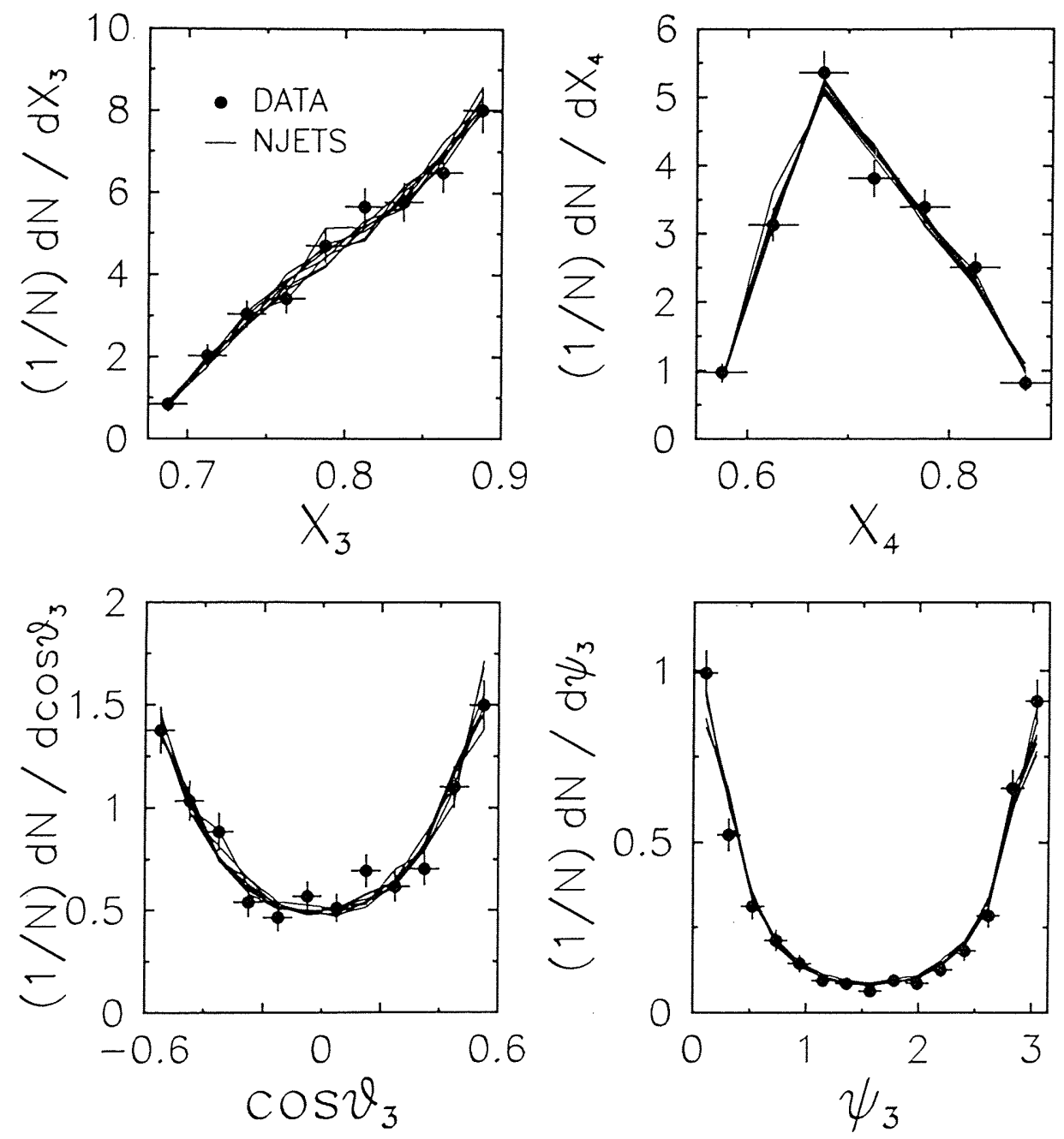

Figure 7.2: The NJETS predictions of the three-body distributions of 3-jet events for various choices of structure functions, $Q^{2}$-scales, and $\Delta R_{\min }$. Eight curves in each figure correspond to the eight choices listed in Table 7.1. Histograms are the measured distribution. (a) $X_{3}$, (b) $X_{4}$, (c) $\cos \theta_{3}$, and (d) $\psi_{3}$. 


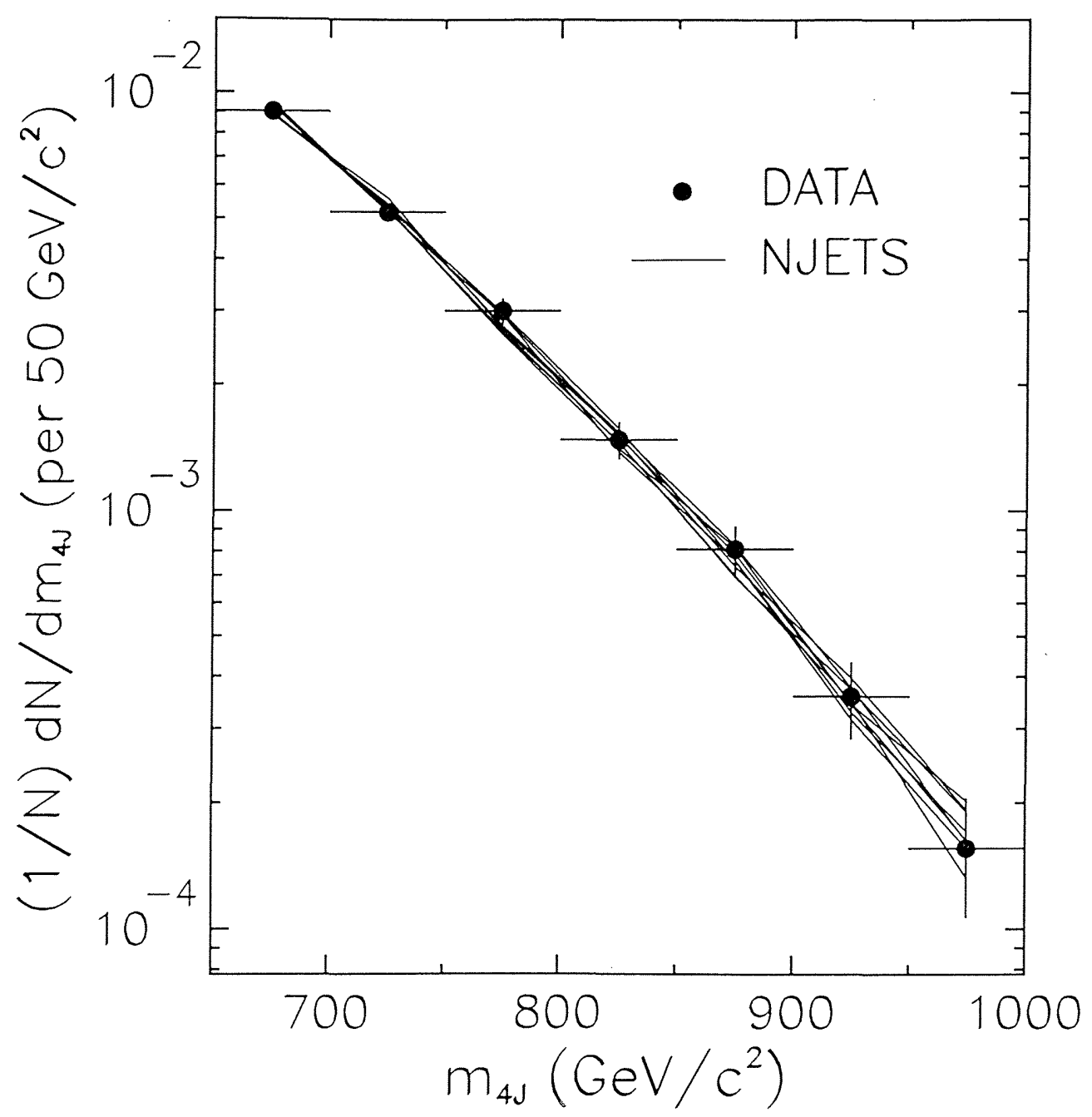

Figure 7.3: The NJETS predictions of the $m_{4 J}$ distribution of 4-jet events for various choices of structure functions, $Q^{2}$-scales, and $\Delta R_{\text {min }}$. Eight curves in the figure correspond to the eight choices listed in Table 7.1. The histogram is the measured $m_{4} J$ distribution. 

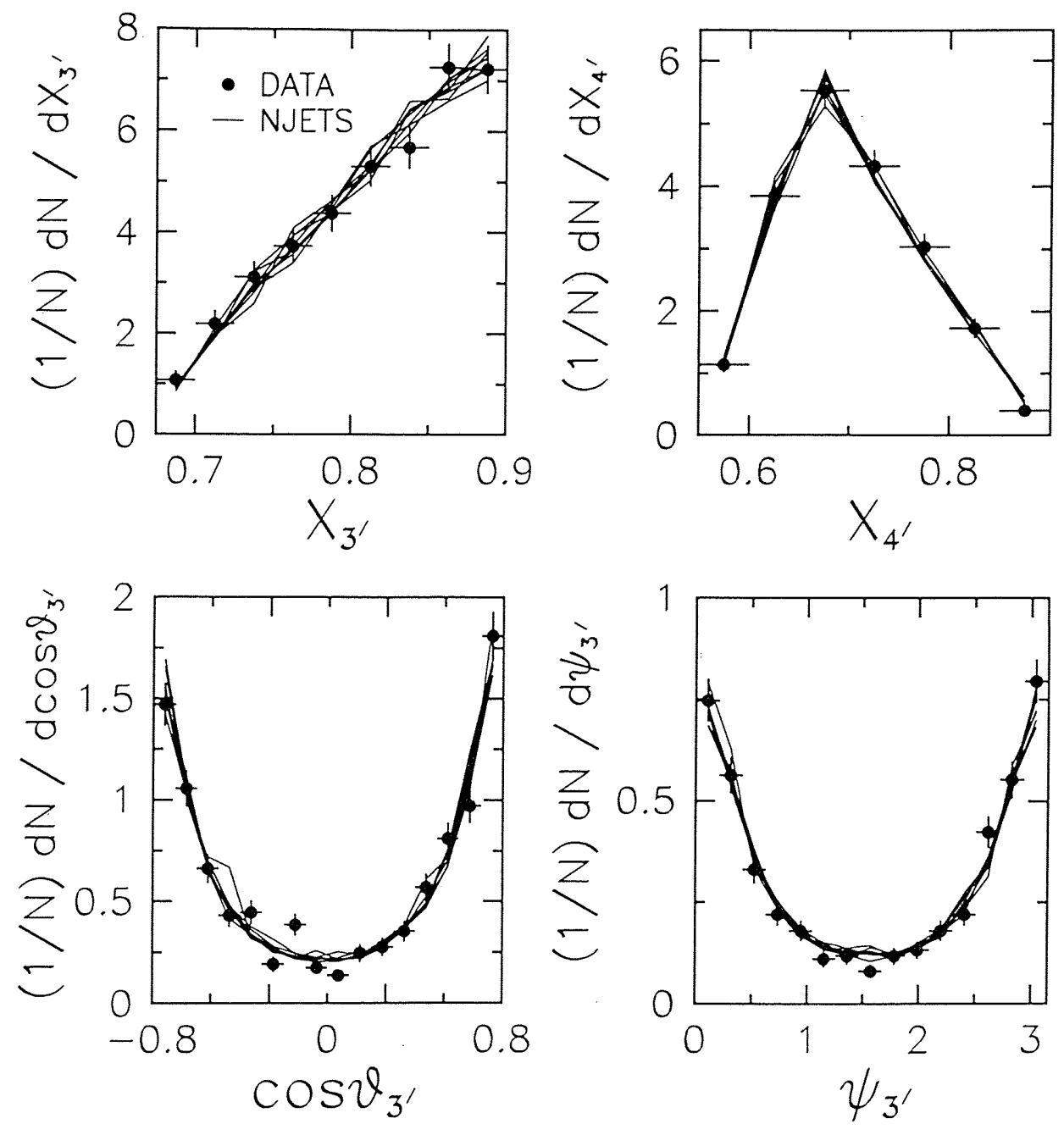

Figure 7.4: The NJETS predictions of the three-body distributions of 4-jet events for various choices of structure functions, $Q^{2}$-scales, and $\Delta R_{\min }$. Eight curves in each figure correspond to the eight choices listed in Table 7.1. Histograms are the measured distribution. (a) $X_{3^{\prime}}$, (b) $X_{4^{\prime}}$, (c) $\cos \theta_{3^{\prime}}$, and (d) $\psi_{3^{\prime}}$. 


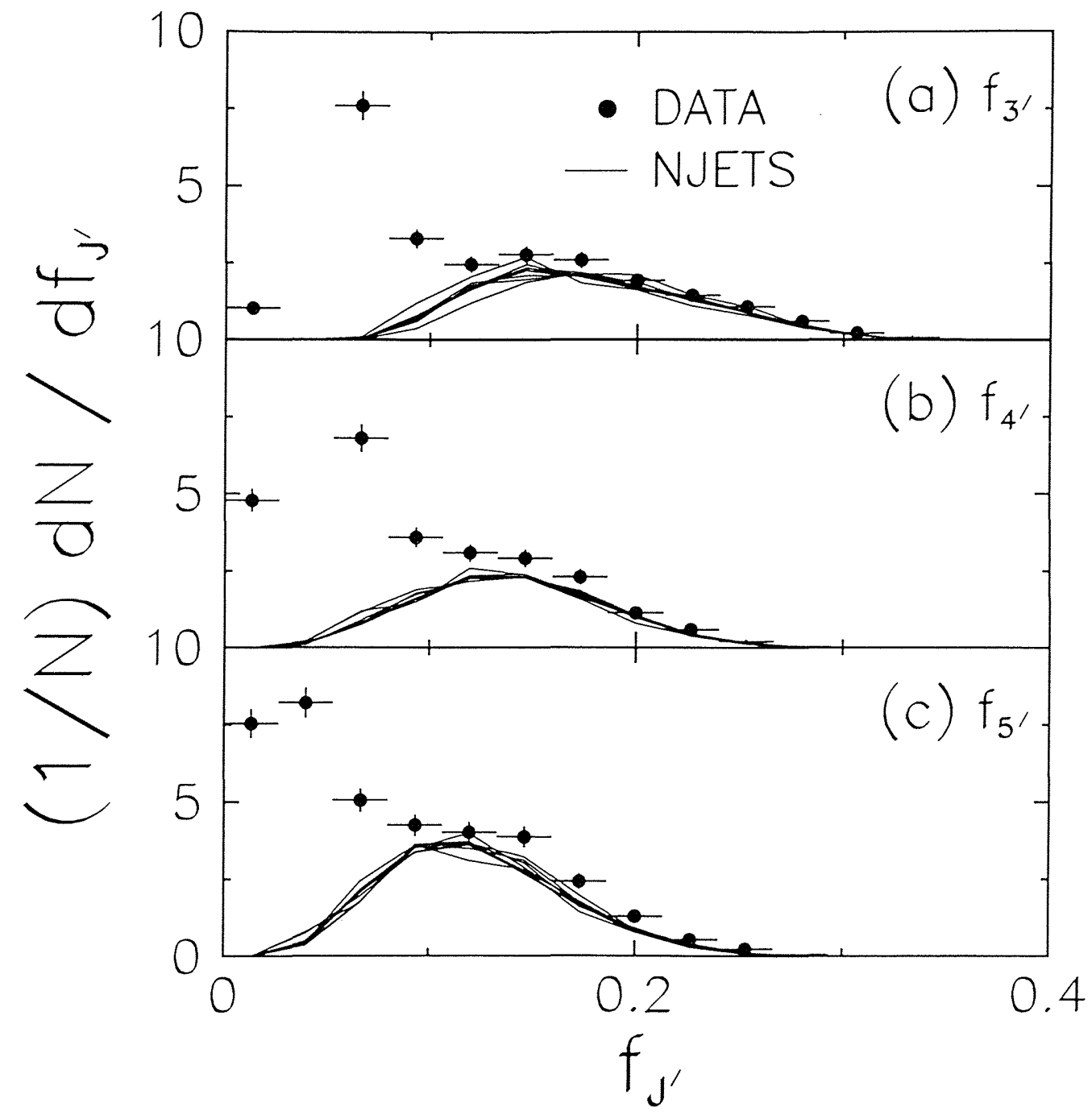

Figure 7.5: The NJETS predictions of the $f_{J^{\prime}}\left(J^{\prime}=3^{\prime}, 4^{\prime}, 5^{\prime}\right)$ distributions of 4-jet events for various choices of structure functions, $Q^{2}$-scales, and $\Delta R_{\min }$. Eight curves in each figure correspond to the eight choices listed in Table 7.1. Histograms are the measured distribution. (a) $f_{3^{\prime}}$, (b) $f_{4^{\prime}}$, and (c) $f_{5^{\prime}}$. 

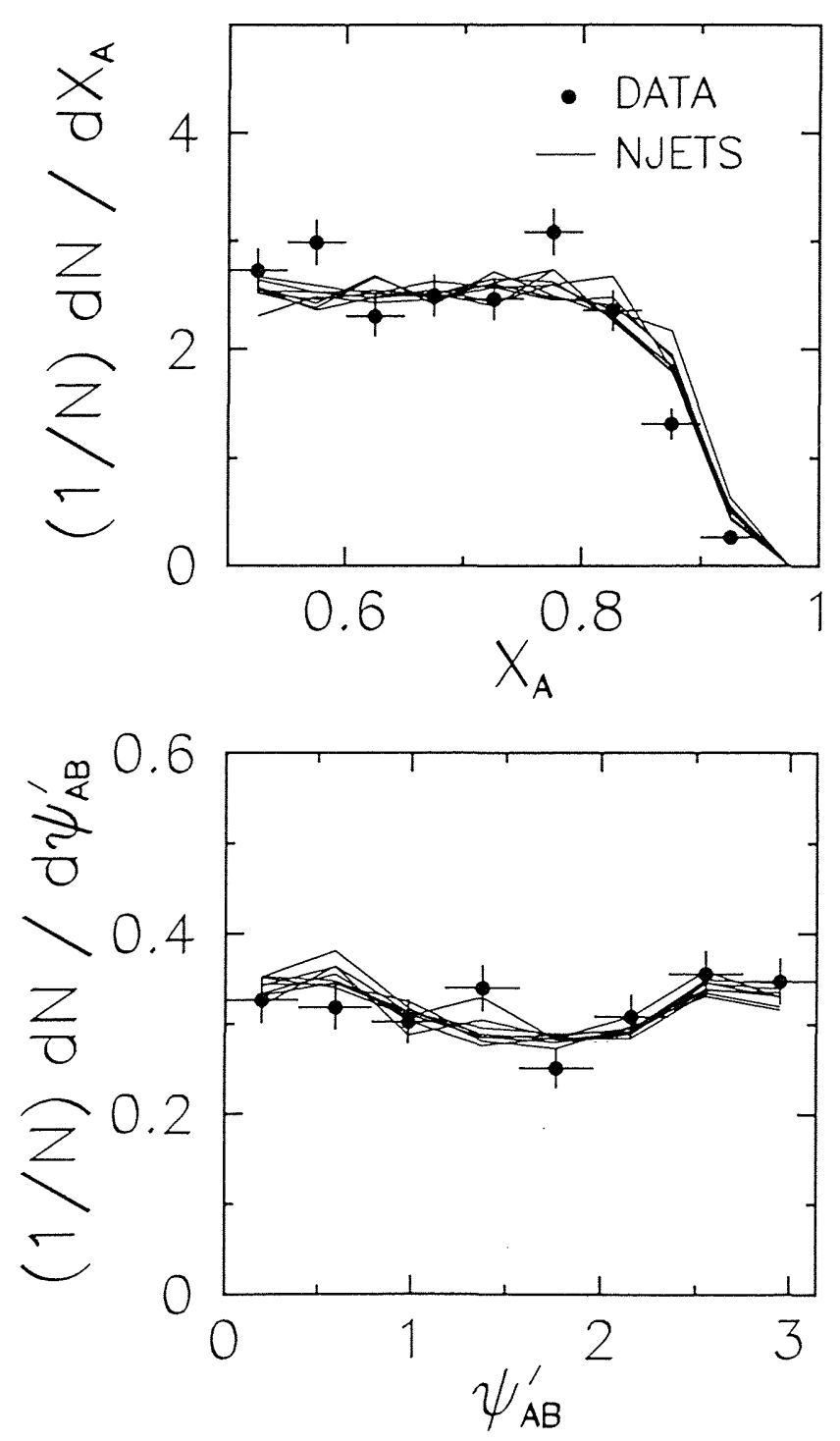

Figure 7.6: The NJETS predictions of the two $(A+B)$-body distributions of 4 -jet events for various choices of structure functions, $Q^{2}$-scales, and $\Delta R_{\min }$. Eight curves in each figure correspond to the eight choices listed in Table 7.1. Histograms are the measured distribution. (a) $X_{A^{\prime}}$, (b) $\psi_{A B}^{\prime}$. 


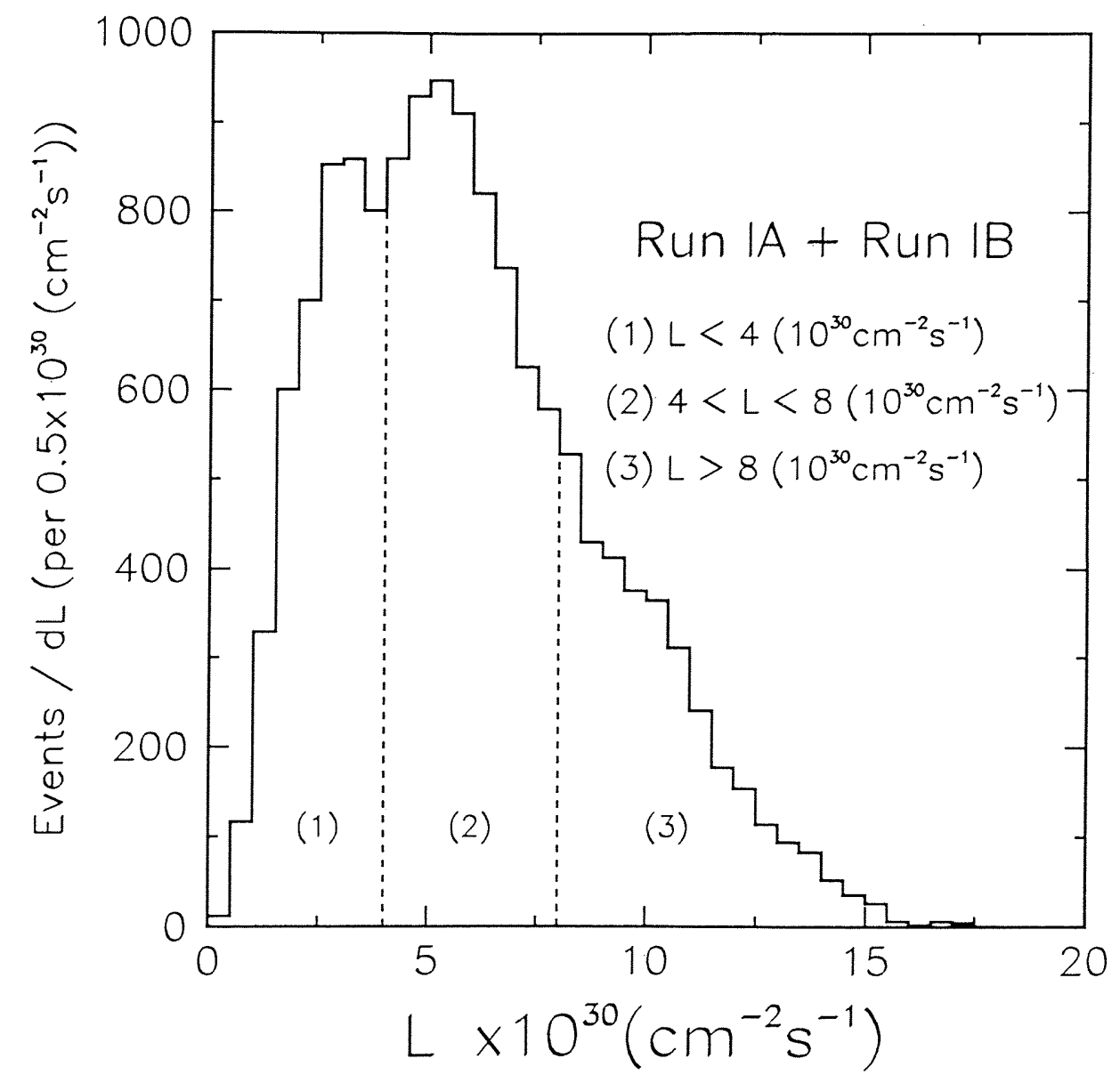

Figure 7.7: The instantaneous luminosity distribution. The multijet data sample was separated into three subsamples containing events detected at low luminosities $(\mathcal{L}<$ $\left.4 \times 10^{30} \mathrm{~cm}^{-2} \mathrm{~s}^{-1}\right)$, intermediate luminosities $\left(4<\mathcal{L}<8 \times 10^{30} \mathrm{~cm}^{-2} \mathrm{~s}^{-1}\right)$, and high luminosities $\left(\mathcal{L}>8 \times 10^{30} \mathrm{~cm}^{-2} \mathrm{~s}^{-1}\right)$. 


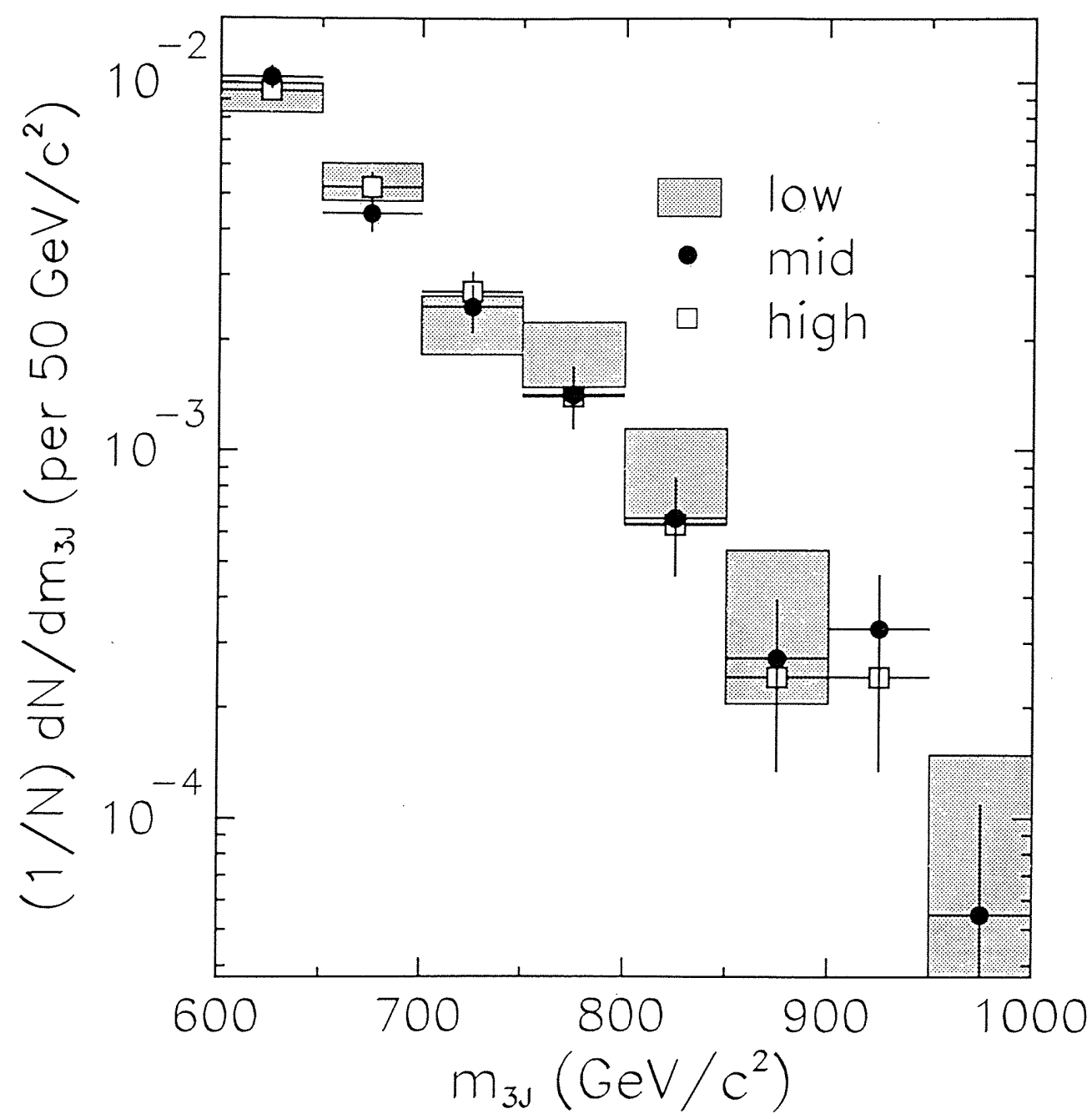

Figure 7.8: The observed $m_{3 J}$ distributions of 3-jet events in three different ranges of instantaneous luminosities. In the figure the histogram corresponds to the lowest luminosity range $\left(\mathcal{L}<4 \times 10^{30} \mathrm{~cm}^{-2} \mathrm{~s}^{-1}\right)$, points correspond to the intermediate luminosity range $\left(4<\mathcal{L}<8 \times 10^{30} \mathrm{~cm}^{-2} \mathrm{~s}^{-1}\right)$, and squares correspond to the highest luminosity range $\left(\mathcal{L}>8 \times 10^{30} \mathrm{~cm}^{-2} \mathrm{~s}^{-1}\right)$, respectively. 

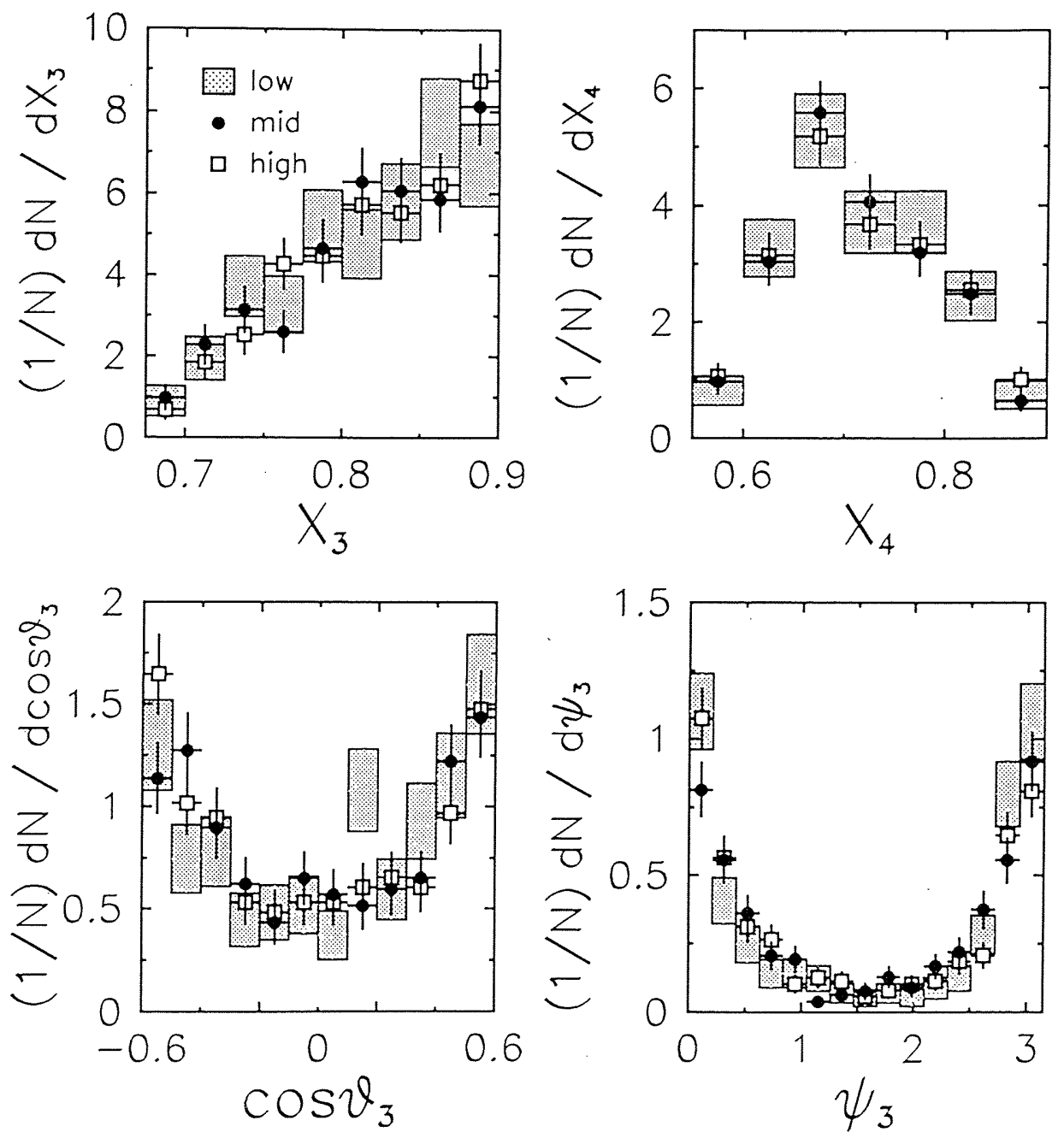

Figure 7.9: The observed three-body distributions of 3 -jet events in three different ranges of instantaneous luminosities. In the figure the histograms correspond to the lowest luminosity range $\left(\mathcal{L}<4 \times 10^{30} \mathrm{~cm}^{-2} \mathrm{~s}^{-1}\right)$, points correspond to the intermediate luminosity range $\left(4<\mathcal{L}<8 \times 10^{30} \mathrm{~cm}^{-2} \mathrm{~s}^{-1}\right)$, and squares correspond to the highest luminosity range $\left(\mathcal{L}>8 \times 10^{30} \mathrm{~cm}^{-2} \mathrm{~s}^{-1}\right)$, respectively. (a) $X_{3}$, (b) $X_{4}$, (c) $\cos \theta_{3}$, and (d) $\psi_{3}$. 


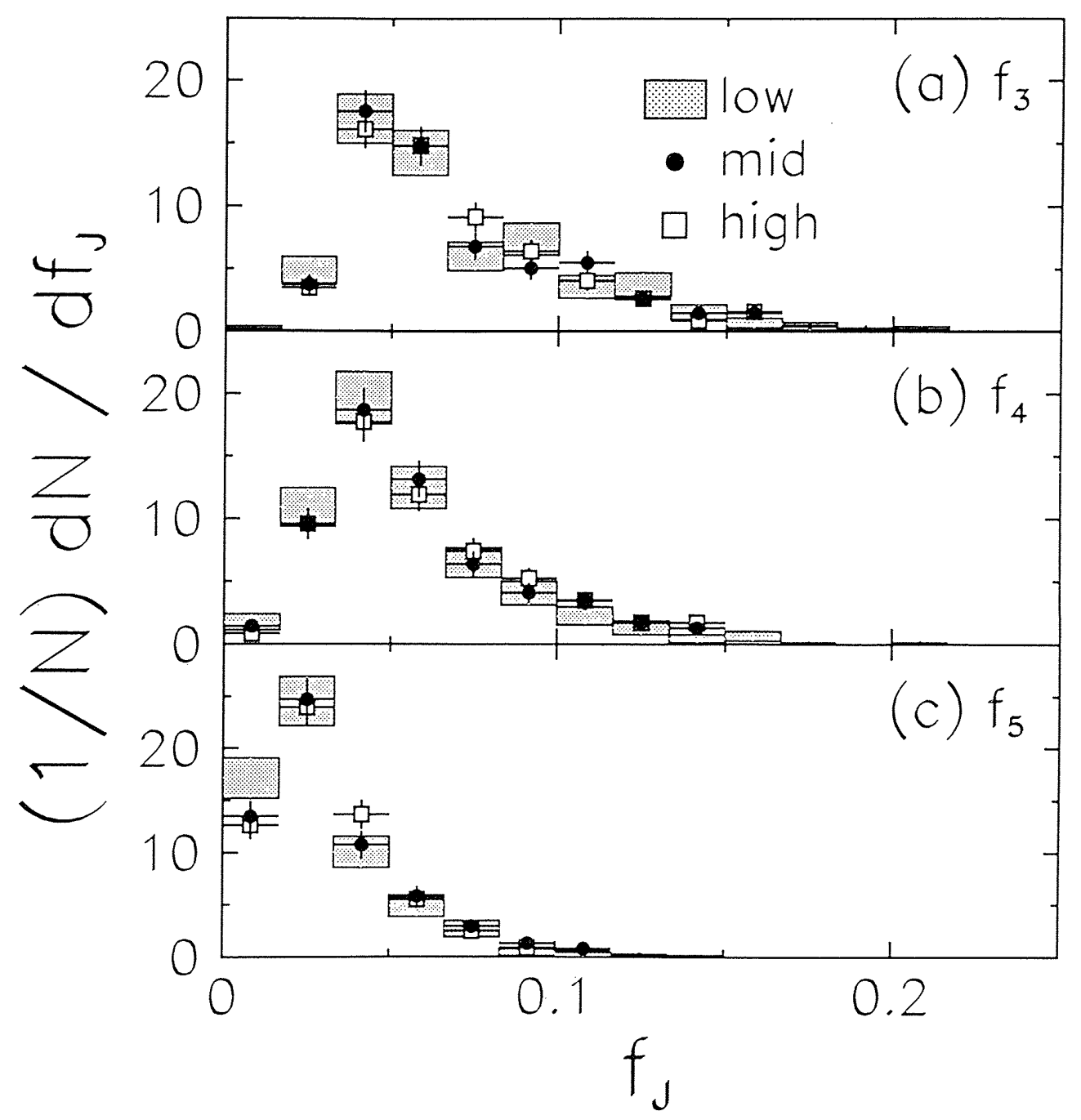

Figure 7.10: The observed $f_{J}(J=3,4,5)$ distributions of 3 -jet events in three different ranges of instantaneous luminosities. In the figure the histograms correspond to the lowest luminosity range $\left(\mathcal{L}<4 \times 10^{30} \mathrm{~cm}^{-2} \mathrm{~s}^{-1}\right)$, points correspond to the intermediate luminosity range $\left(4<\mathcal{L}<8 \times 10^{30} \mathrm{~cm}^{-2} \mathrm{~s}^{-1}\right)$, and squares correspond to the highest luminosity range $\left(\mathcal{L}>8 \times 10^{30} \mathrm{~cm}^{-2} \mathrm{~s}^{-1}\right)$, respectively. (a) $f_{3},(\mathrm{~b}) f_{4}$, and (c) $f_{5}$. 


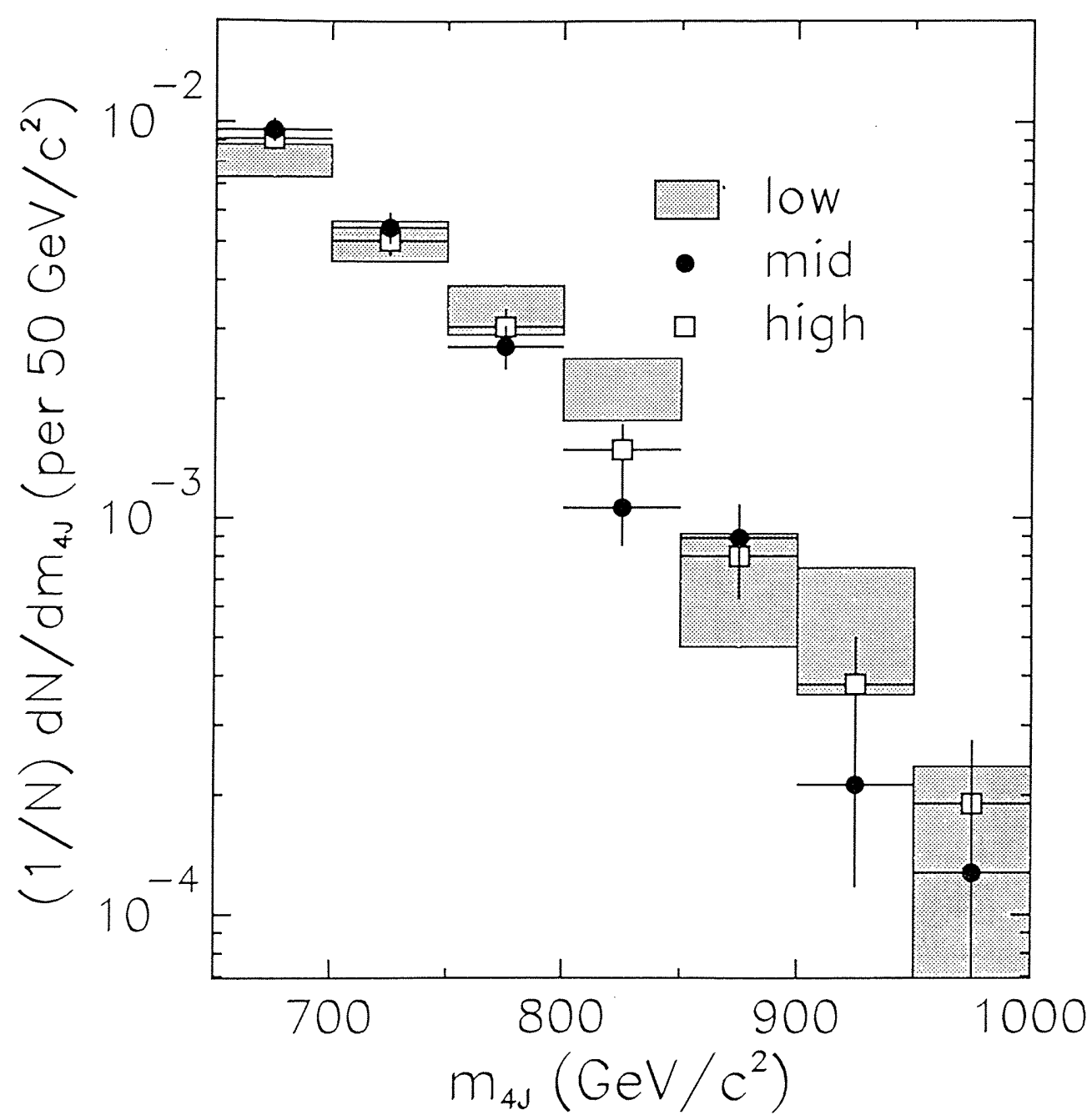

Figure 7.11: The observed $m_{4 J}$ distributions in three different ranges of instantaneous luminosities. In the figure the histogram corresponds to the lowest luminosity range $\left(\mathcal{L}<4 \times 10^{30} \mathrm{~cm}^{-2} \mathrm{~s}^{-1}\right)$, points correspond to the intermediate luminosity range $(4<$ $\left.\mathcal{L}<8 \times 10^{30} \mathrm{~cm}^{-2} \mathrm{~s}^{-1}\right)$, and squares correspond to the highest luminosity range $(\mathcal{L}>$ $8 \times 10^{30} \mathrm{~cm}^{-2} \mathrm{~s}^{-1}$ ), respectively. 

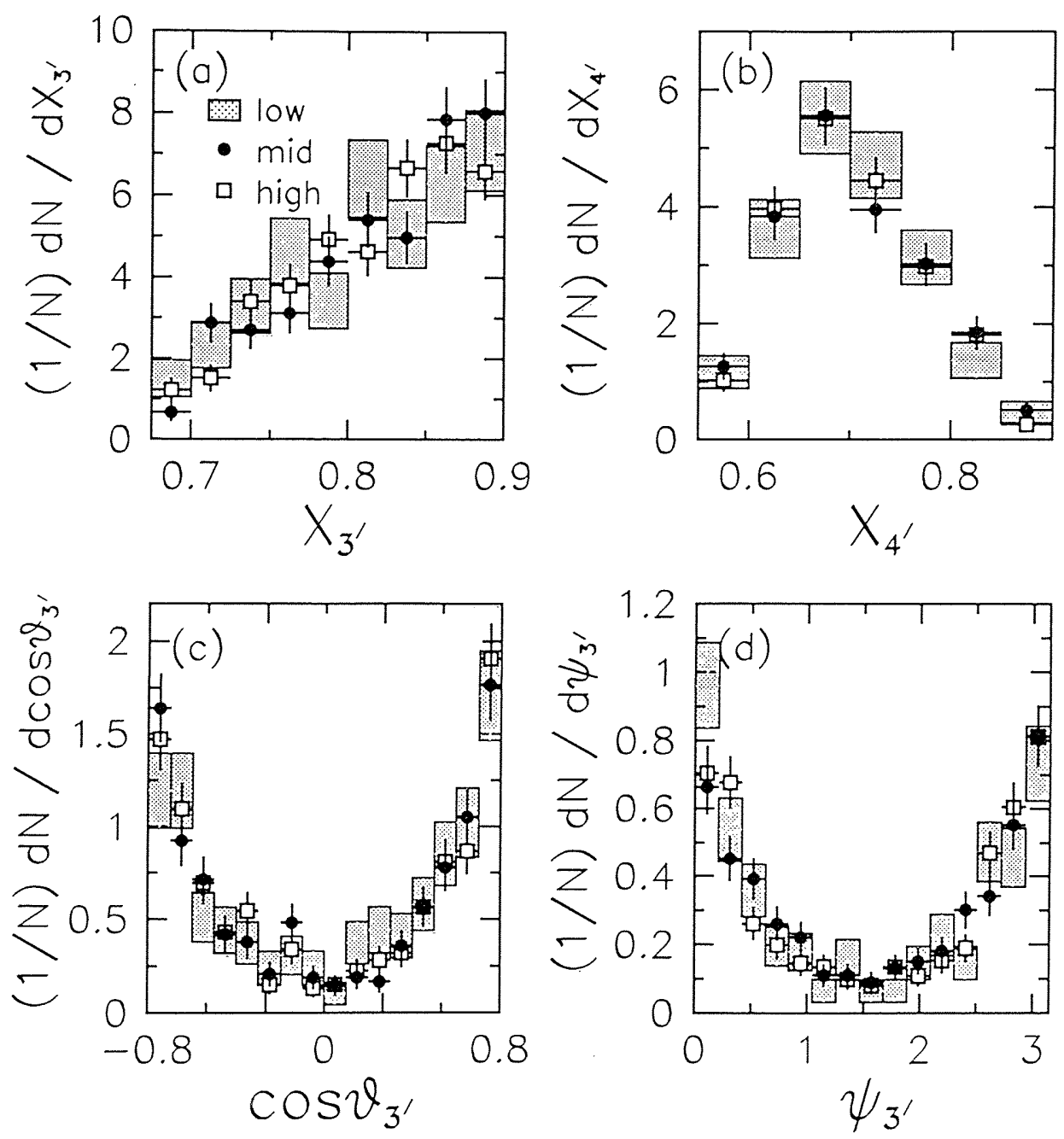

Figure 7.12: The observed three-body distributions of 4-jet events in three different ranges of instantaneous luminosities. In the figure the histograms correspond to the lowest luminosity range $\left(\mathcal{L}<4 \times 10^{30} \mathrm{~cm}^{-2} \mathrm{~s}^{-1}\right)$, points correspond to the intermediate luminosity range $\left(4<\mathcal{L}<8 \times 10^{30} \mathrm{~cm}^{-2} \mathrm{~s}^{-1}\right)$, and squares correspond to the highest luminosity range $\left(\mathcal{L}>8 \times 10^{30} \mathrm{~cm}^{-2} \mathrm{~s}^{-1}\right)$, respectively. (a) $X_{3^{\prime}}$, (b) $X_{4^{\prime}}$, (c) $\cos \theta_{3^{\prime}}$, and (d) $\psi_{3^{\prime}}$. 


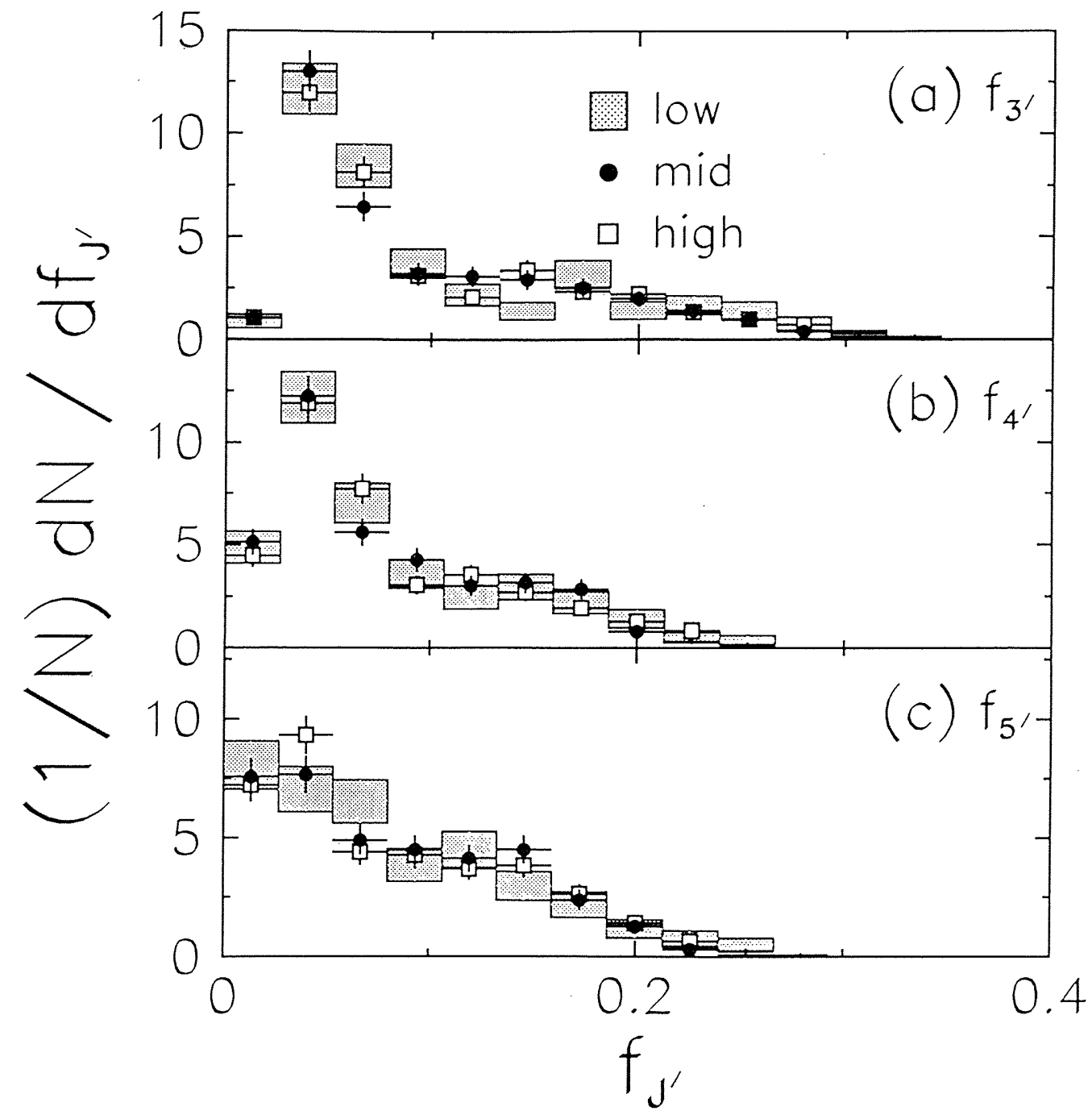

Figure 7.13: The observed $f_{J^{\prime}}\left(J^{\prime}=3^{\prime}, 4^{\prime}, 5^{\prime}\right)$ distributions of 4 -jet events in three different ranges of instantaneous luminosities. In the figure the histograms correspond to the lowest luminosity range $\left(\mathcal{L}<4 \times 10^{30} \mathrm{~cm}^{-2} \mathrm{~s}^{-1}\right)$, points correspond to the intermediate luminosity range $\left(4<\mathcal{L}<8 \times 10^{30} \mathrm{~cm}^{-2} \mathrm{~s}^{-1}\right)$, and squares correspond to the highest luminosity range $\left(\mathcal{L}>8 \times 10^{30} \mathrm{~cm}^{-2} \mathrm{~s}^{-1}\right)$, respectively. (a) $f_{3^{\prime}}$, (b) $f_{4^{\prime}}$, and (c) $f_{5^{\prime}}$. 

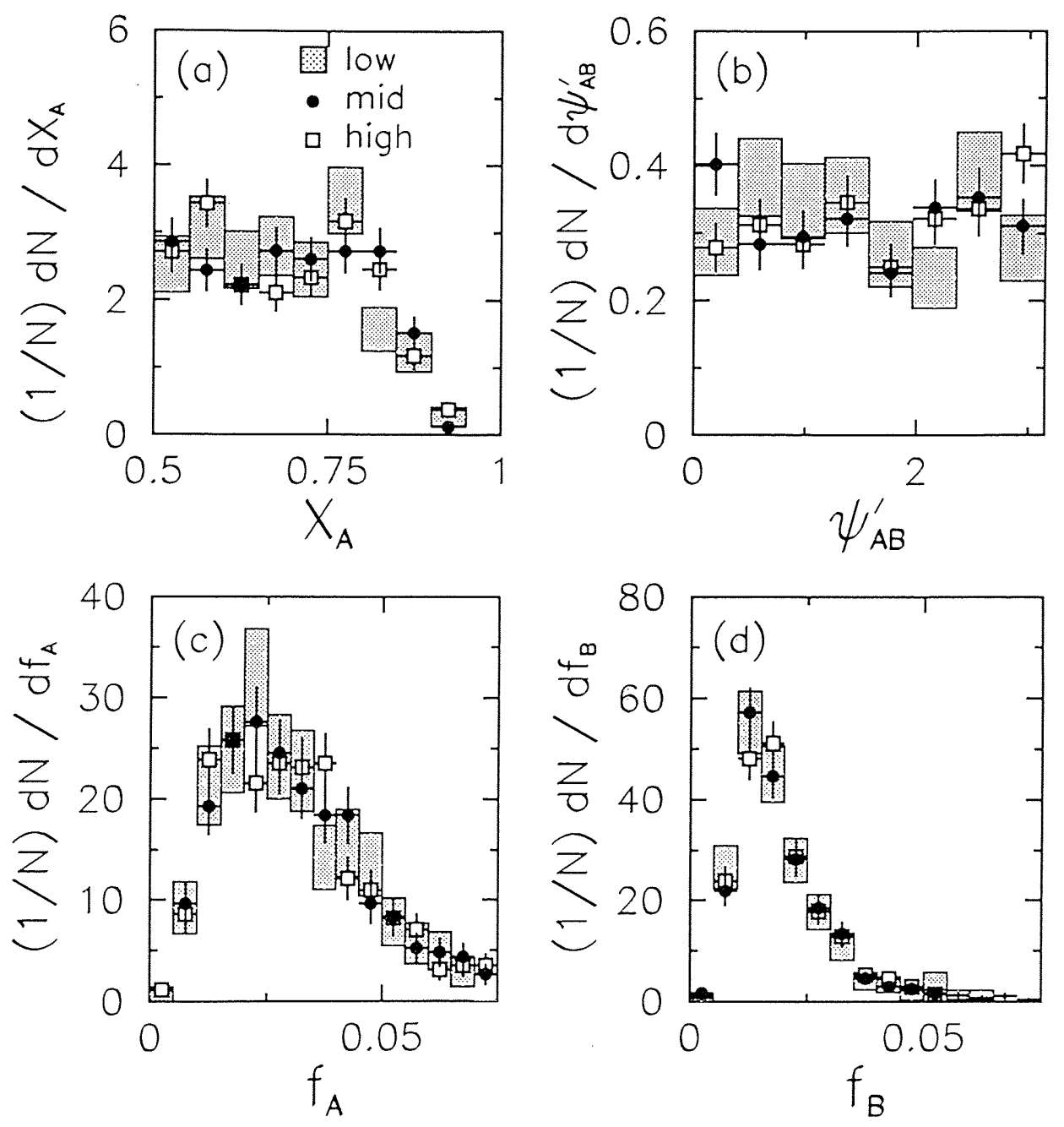

Figure 7.14: The observed two $(A+B)$-body distributions of 4-jet events in three different ranges of instantaneous luminosities. In the figure the histograms correspond to the lowest luminosity range $\left(\mathcal{L}<4 \times 10^{30} \mathrm{~cm}^{-2} \mathrm{~s}^{-1}\right)$, points correspond to the intermediate luminosity range $\left(4<\mathcal{L}<8 \times 10^{30} \mathrm{~cm}^{-2} \mathrm{~s}^{-1}\right)$, and squares correspond to the highest luminosity range $\left(\mathcal{L}>8 \times 10^{30} \mathrm{~cm}^{-2} \mathrm{~s}^{-1}\right)$, respectively. (a) $X_{A}$, (b) $\psi_{A B}^{\prime}$, (c) $f_{A}$, and $(\mathrm{d}) f_{B}$. 


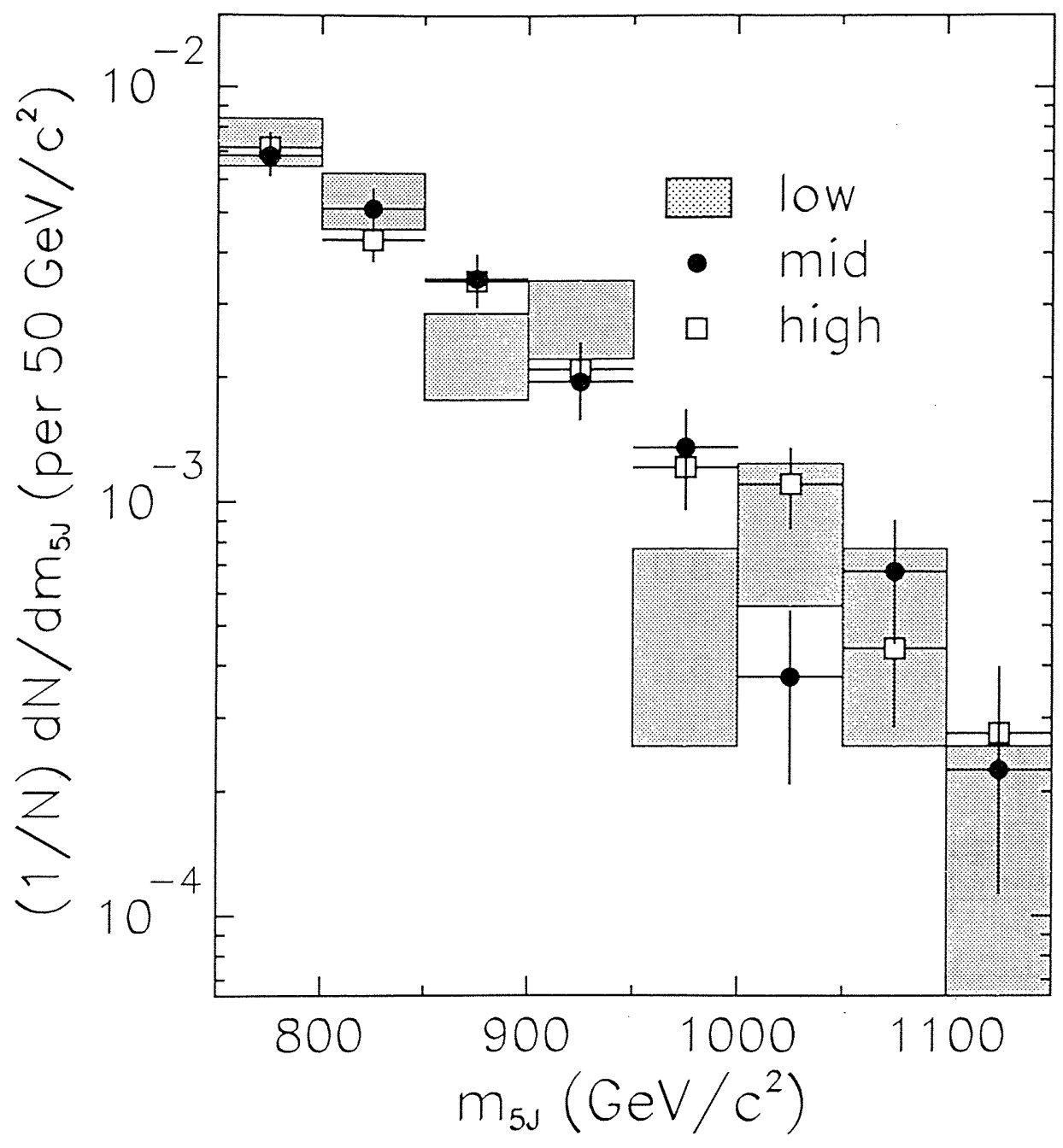

Figure 7.15: The observed $m_{5 J}$ distributions in three different ranges of instantaneous luminosities. In the figure the histogram corresponds to the lowest luminosity range $\left(\mathcal{L}<4 \times 10^{30} \mathrm{~cm}^{-2} \mathrm{~s}^{-1}\right)$, points correspond to the intermediate luminosity range $(4<$ $\left.\mathcal{L}<8 \times 10^{30} \mathrm{~cm}^{-2} \mathrm{~s}^{-1}\right)$, and squares correspond to the highest luminosity range $(\mathcal{L}>$ $8 \times 10^{30} \mathrm{~cm}^{-2} \mathrm{~s}^{-1}$ ), respectively. 

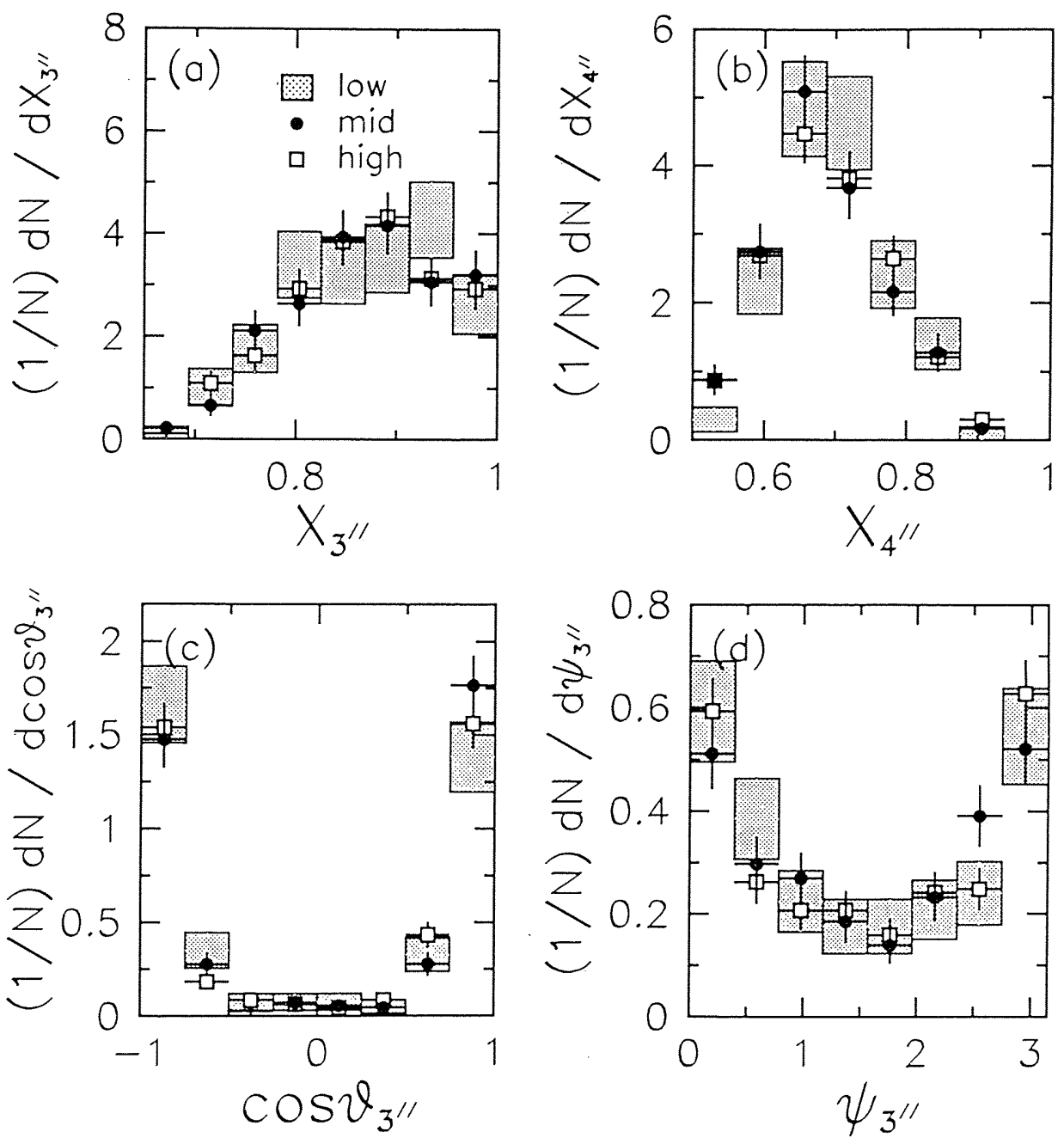

Figure 7.16: The observed three-body distributions of 5-jet events in three different ranges of instantaneous luminosities. In the figure the histograms correspond to the lowest luminosity range $\left(\mathcal{L}<4 \times 10^{30} \mathrm{~cm}^{-2} \mathrm{~s}^{-1}\right)$, points correspond to the intermediate luminosity range $\left(4<\mathcal{L}<8 \times 10^{30} \mathrm{~cm}^{-2} \mathrm{~s}^{-1}\right)$, and squares correspond to the highest luminosity range $\left(\mathcal{L}>8 \times 10^{30} \mathrm{~cm}^{-2} \mathrm{~s}^{-1}\right)$, respectively. (a) $X_{3^{\prime \prime}}$, (b) $X_{4^{\prime \prime}}$, (c) $\cos \theta_{3^{\prime \prime}}$, and (d) $\psi_{3^{\prime \prime}}$. 


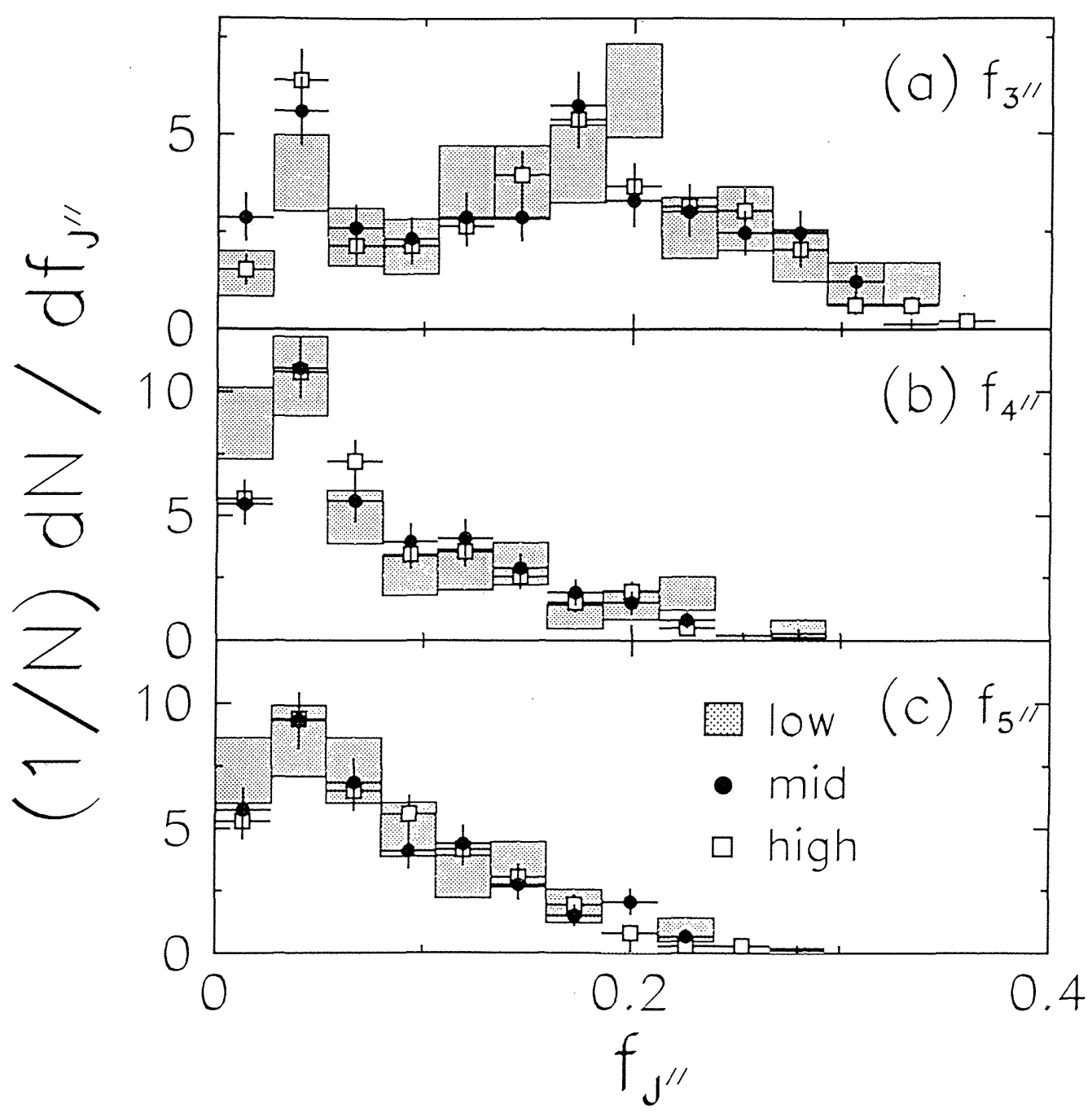

Figure 7.17: The observed $f_{J^{\prime \prime}}\left(J^{\prime \prime}=3^{\prime \prime}, 4^{\prime \prime}, 5^{\prime \prime}\right)$ distributions of 5-jet events in three different ranges of instantaneous luminosities. In the figure the histograms correspond to the lowest luminosity range $\left(\mathcal{L}<4 \times 10^{30} \mathrm{~cm}^{-2} \mathrm{~s}^{-1}\right)$, points correspond to the intermediate luminosity range $\left(4<\mathcal{L}<8 \times 10^{30} \mathrm{~cm}^{-2} \mathrm{~s}^{-1}\right)$, and squares correspond to the highest luminosity range $\left(\mathcal{L}>8 \times 10^{30} \mathrm{~cm}^{-2} \mathrm{~s}^{-1}\right)$, respectively. (a) $f_{3^{\prime \prime}},(\mathrm{b}) f_{4^{\prime \prime}}$, and (c) $f_{5 \prime \prime}$ 

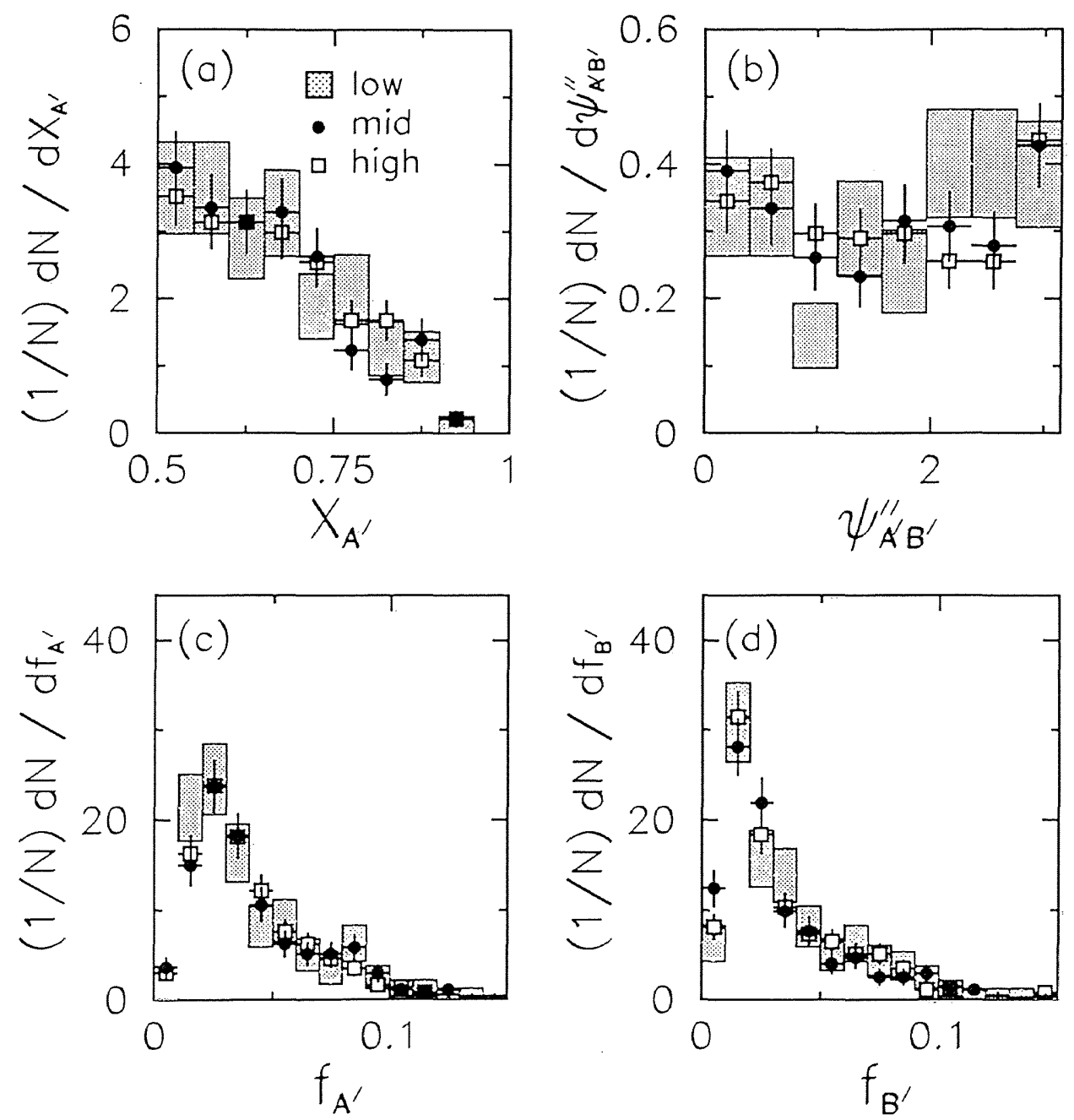

Figure 7.18: The observed two $\left(A^{\prime}+B^{\prime}\right)$-body distributions of 5 -jet events in three different ranges of instantaneous luminosities. In the figure the histograms correspond to the lowest luminosity range $\left(\mathcal{L}<4 \times 10^{30} \mathrm{~cm}^{-2} \mathrm{~s}^{-1}\right)$, points correspond to the intermediate luminosity range $\left(4<\mathcal{L}<8 \times 10^{30} \mathrm{~cm}^{-2} \mathrm{~s}^{-1}\right)$, and squares correspond to the highest luminosity range $\left(\mathcal{L}>8 \times 10^{30} \mathrm{~cm}^{-2} \mathrm{~s}^{-1}\right)$, respectively. (a) $X_{A^{\prime}}$, (b) $\psi_{A^{\prime} B^{\prime}}^{\prime \prime}$, (c) $f_{A^{\prime}}$, and $(\mathrm{d}) f_{B^{\prime}}$. 

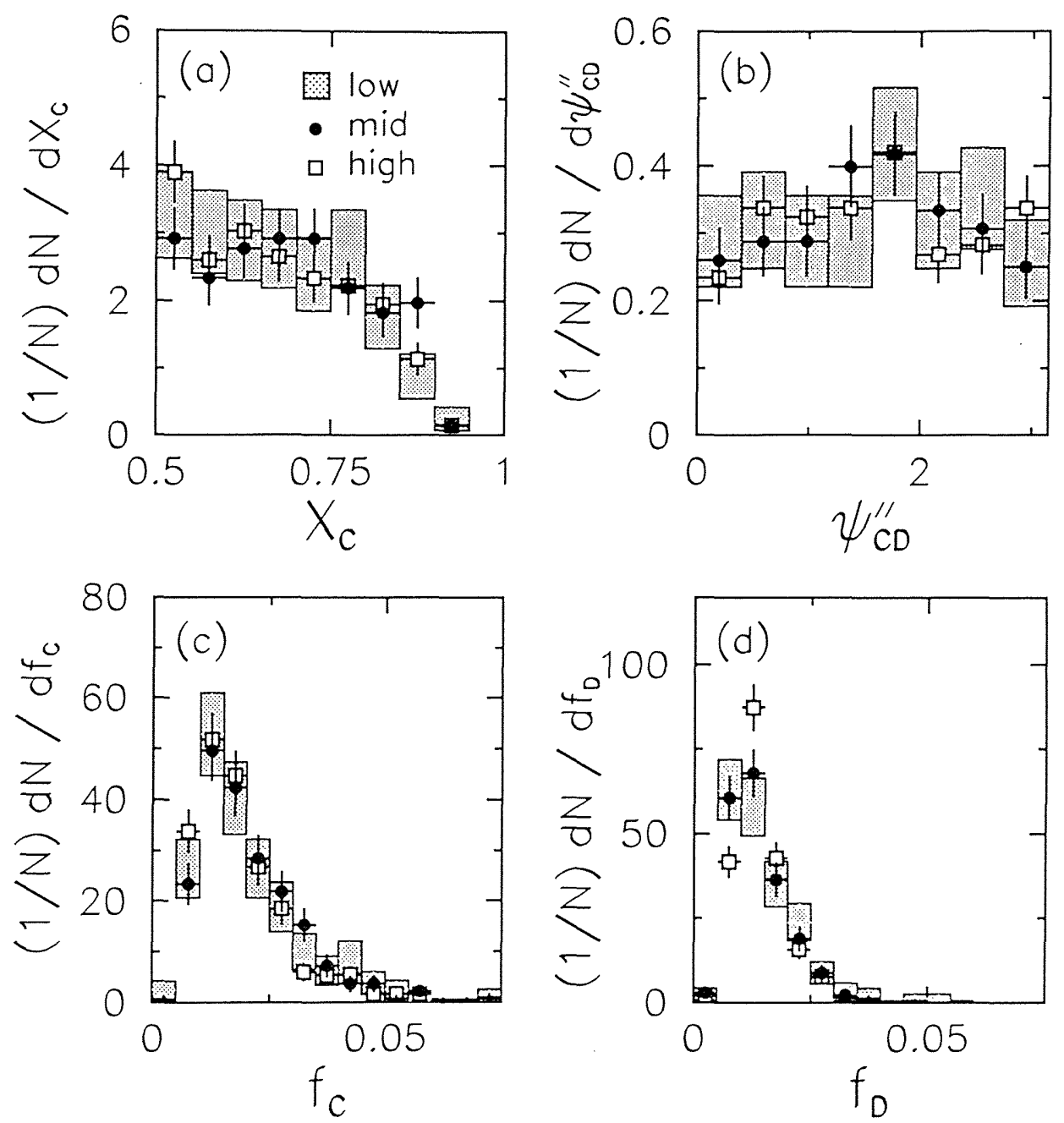

Figure 7.19: The observed two $(C+D)$-body distributions of 5 -jet events in three different ranges of instantaneous luminosities. In the figure the histograms correspond to the lowest luminosity range $\left(\mathcal{L}<4 \times 10^{30} \mathrm{~cm}^{-2} \mathrm{~s}^{-1}\right)$, points correspond to the intermediate luminosity range $\left(4<\mathcal{L}<8 \times 10^{30} \mathrm{~cm}^{-2} \mathrm{~s}^{-1}\right)$, and squares correspond to the highest luminosity range $\left(\mathcal{L}>8 \times 10^{30} \mathrm{~cm}^{-2} \mathrm{~s}^{-1}\right)$, respectively. (a) $X_{C}$, (b) $\psi_{C D}^{\prime \prime}$, (c) $f_{C}$, and (d) $f_{D}$. 


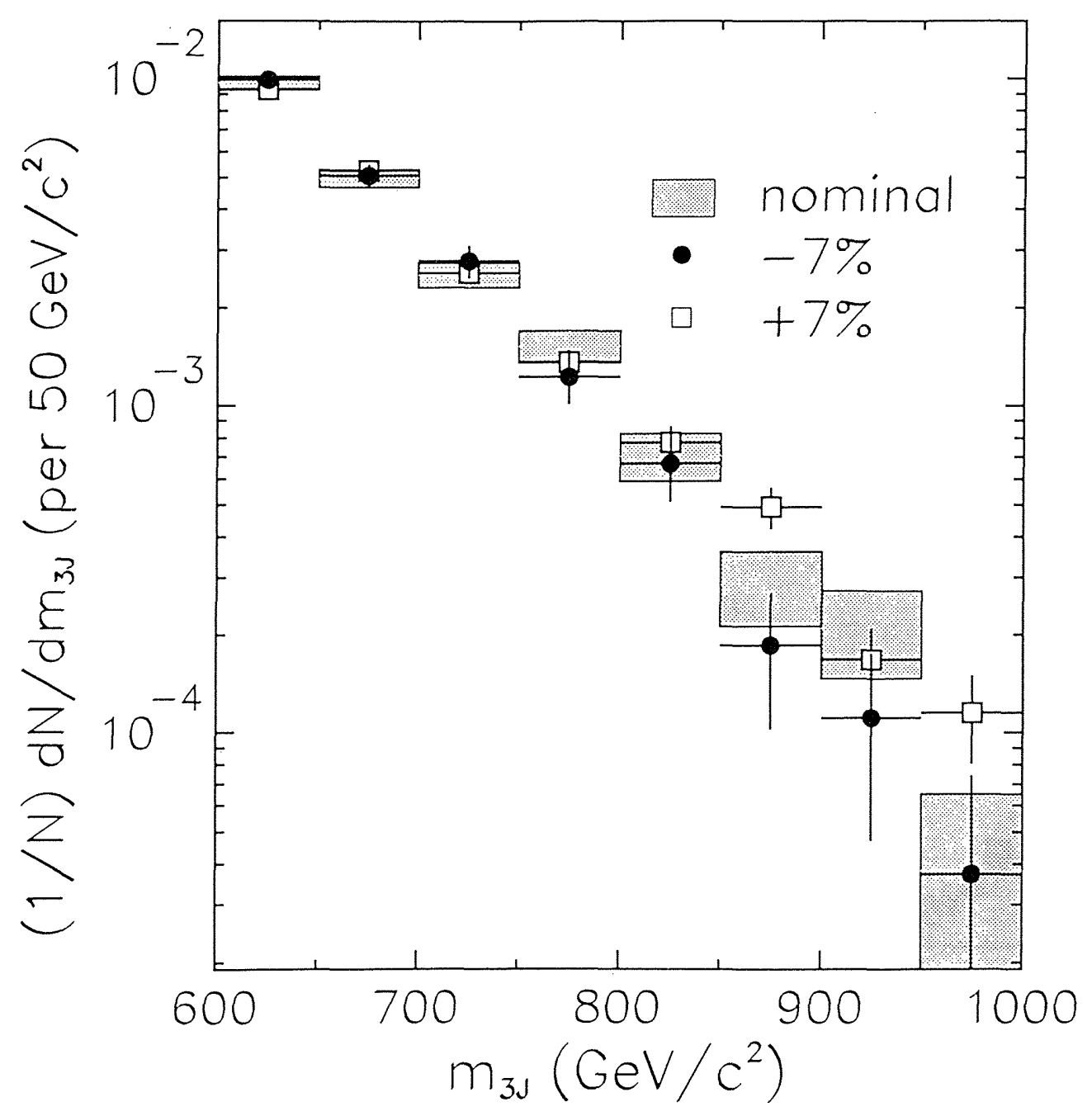

Figure 7.20: The observed $m_{3 J}$ distributions for three data samples in which the jet energies were scaled by $-7 \%, 0 \%$, and $+7 \%$. In the figure the histograms corresponds to events with the nominal energy scale, points and squares correspond to events with energies shifted by $-7 \%$ and $-7 \%$, respectively. 

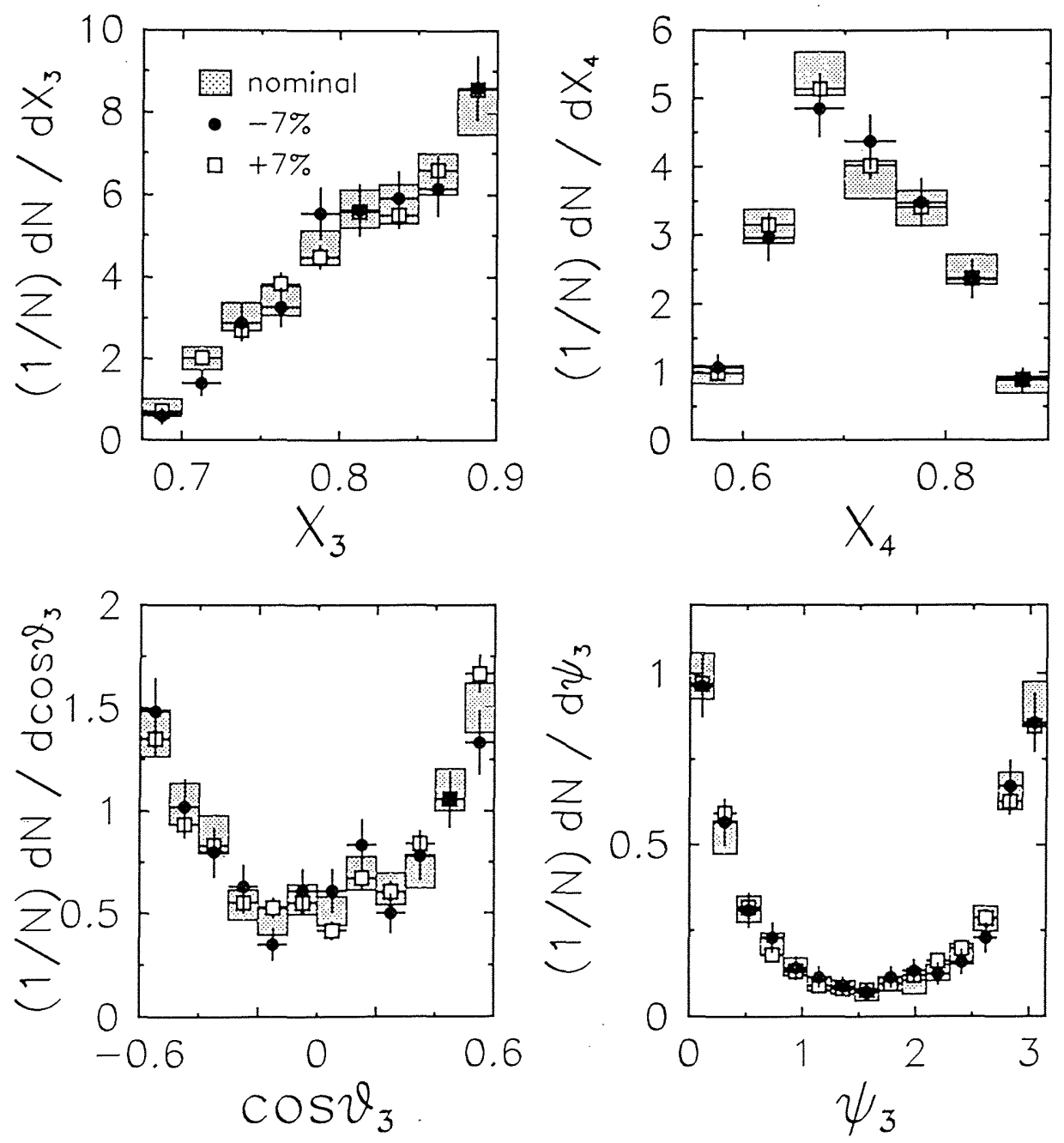

Figure 7.21: The observed three-body distributions of 3-jet events for three data samples in which the jet energies were scaled by $-7 \%, 0 \%$, and $+7 \%$. In the figure histograms correspond to events with the nominal energy scale, points and squares correspond to events with energies shifted by $-7 \%$ and $-7 \%$, respectively. (a) $X_{3}$, (b) $X_{4},(\mathrm{c}) \cos \theta_{3}$, and (d) $\psi_{3}$. 


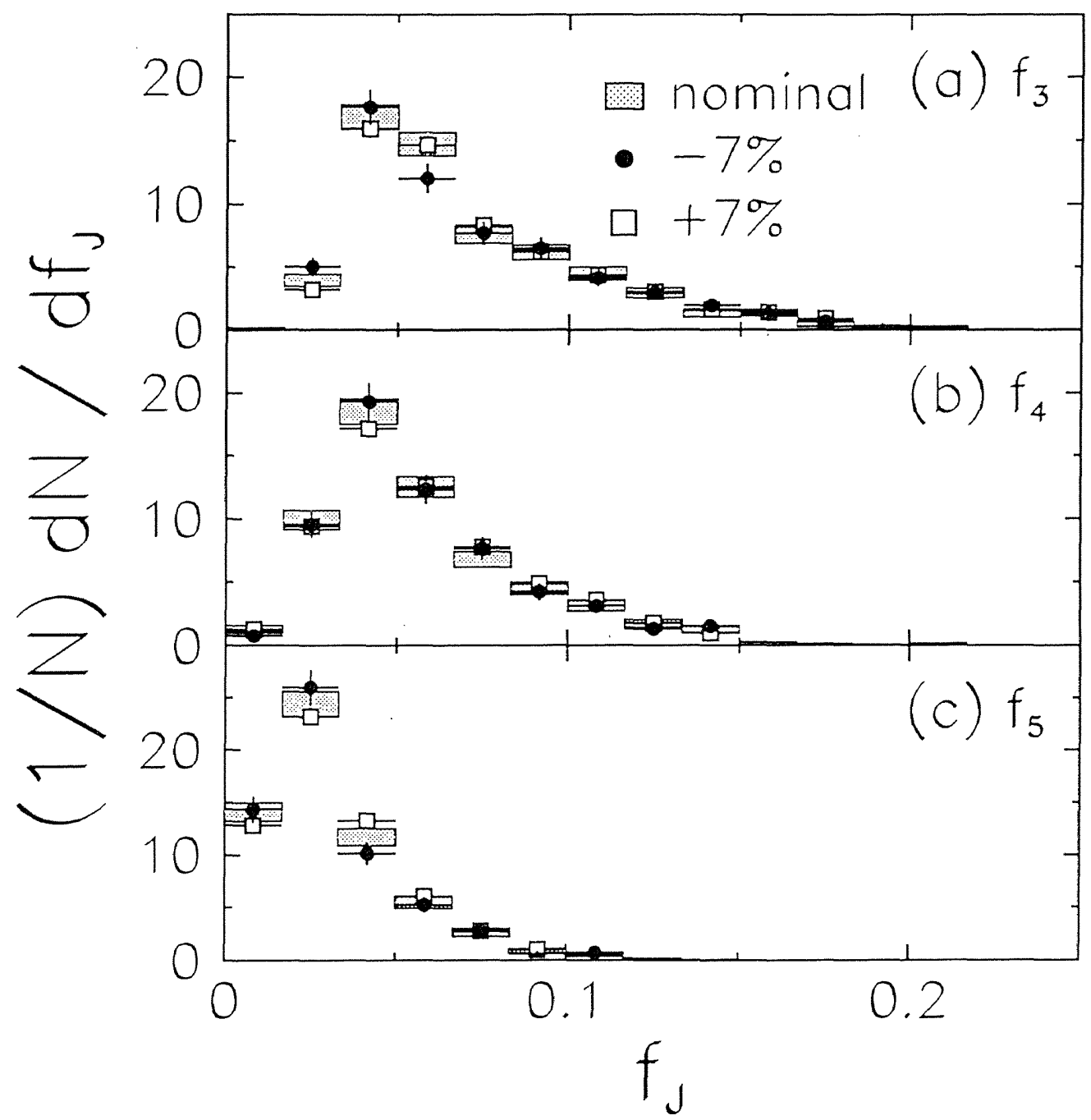

Figure 7.22: The observed $f_{J}(J=3,4,5)$ distributions of 3 -jet events for three data samples in which the jet energies were scaled by $-7 \%, 0 \%$, and $+7 \%$. In the figure histograms correspond to events with the nominal energy scale, points and squares correspond to events with energies shifted by $-7 \%$ and $-7 \%$, respectively. (a) $f_{3}$, (b) $f_{4}$, and $(\mathrm{c}) f_{5}$. 


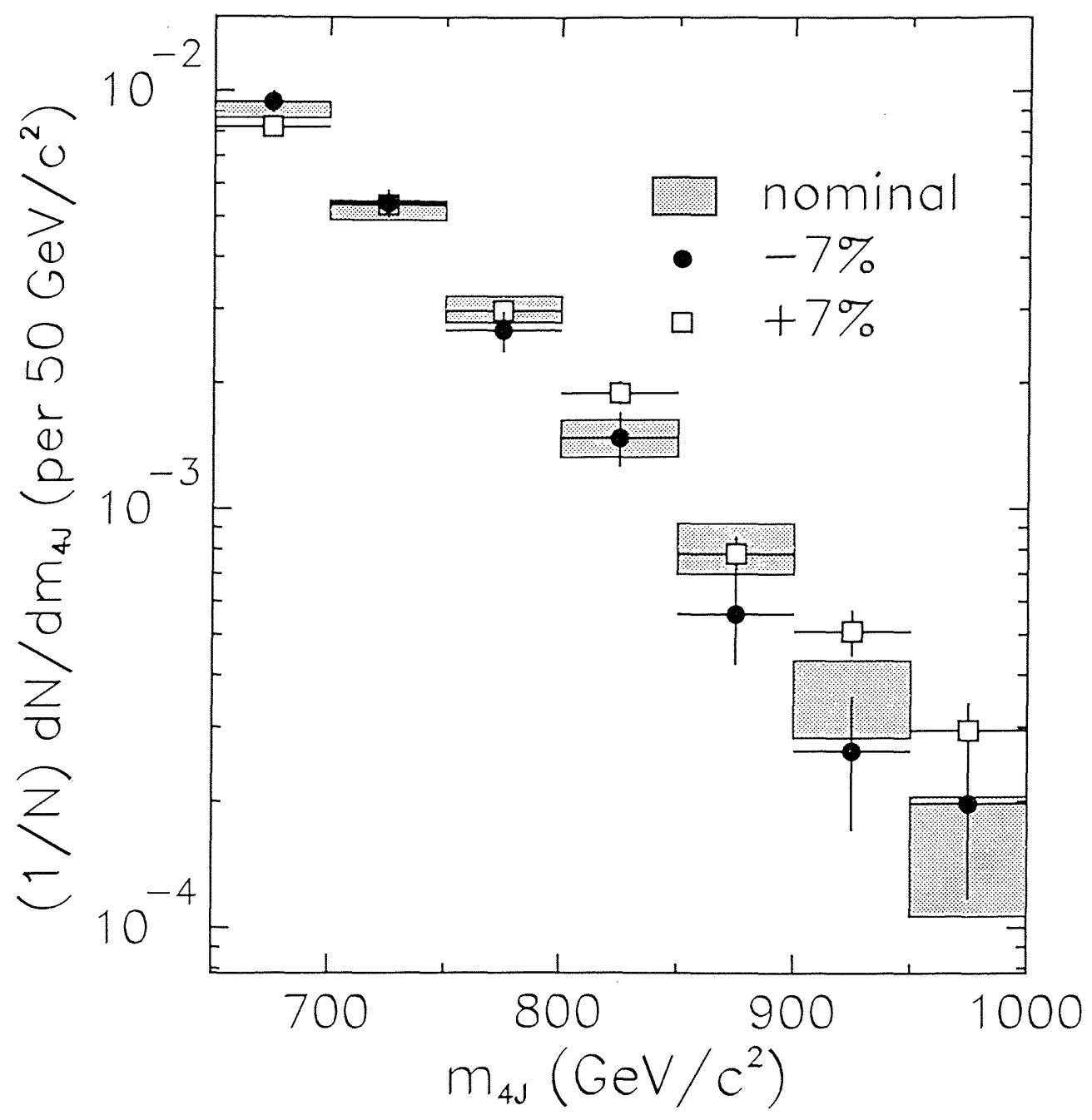

Figure 7.23: The observed $m_{4 J}$ distributions for three data samples in which the jet energies were scaled by $-7 \%, 0 \%$, and $+7 \%$. In the figure the histogram corresponds to events with the nominal energy scale, points and squares correspond to events with energies shifted by $-7 \%$ and $-7 \%$, respectively. 

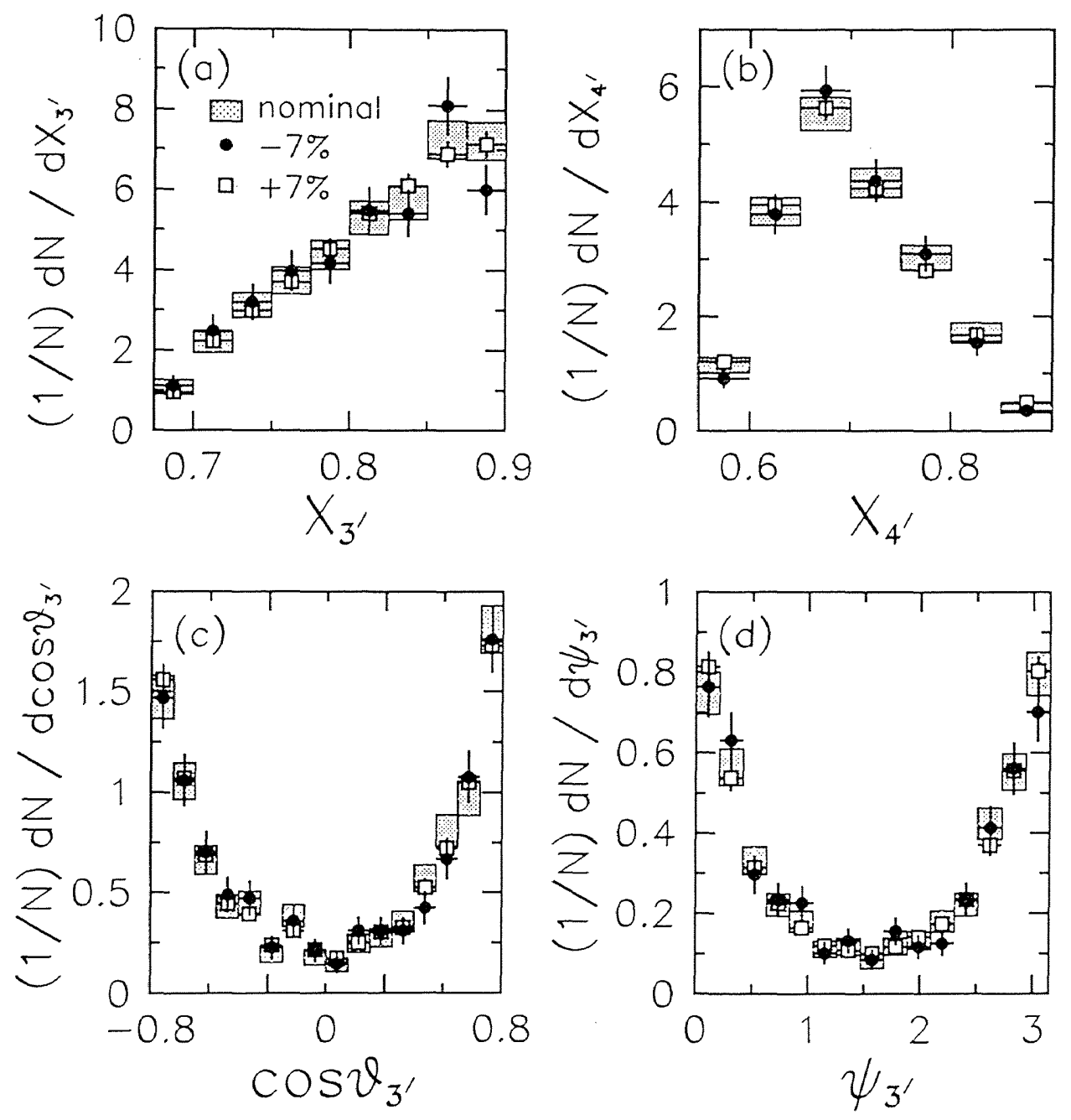

Figure 7.24: The observed three-body distributions of 4-jet events for three data samples in which the jet energies were scaled by $-7 \%, 0 \%$, and $+7 \%$. In the figure the histograms correspond to events with the nominal energy scale, points and squares correspond to events with energies shifted by $-7 \%$ and $-7 \%$, respectively. (a) $X_{3^{\prime}}$, (b) $X_{4^{\prime}},(\mathrm{c}) \cos \theta_{3^{\prime}}$, and (d) $\psi_{3^{\prime}}$ 


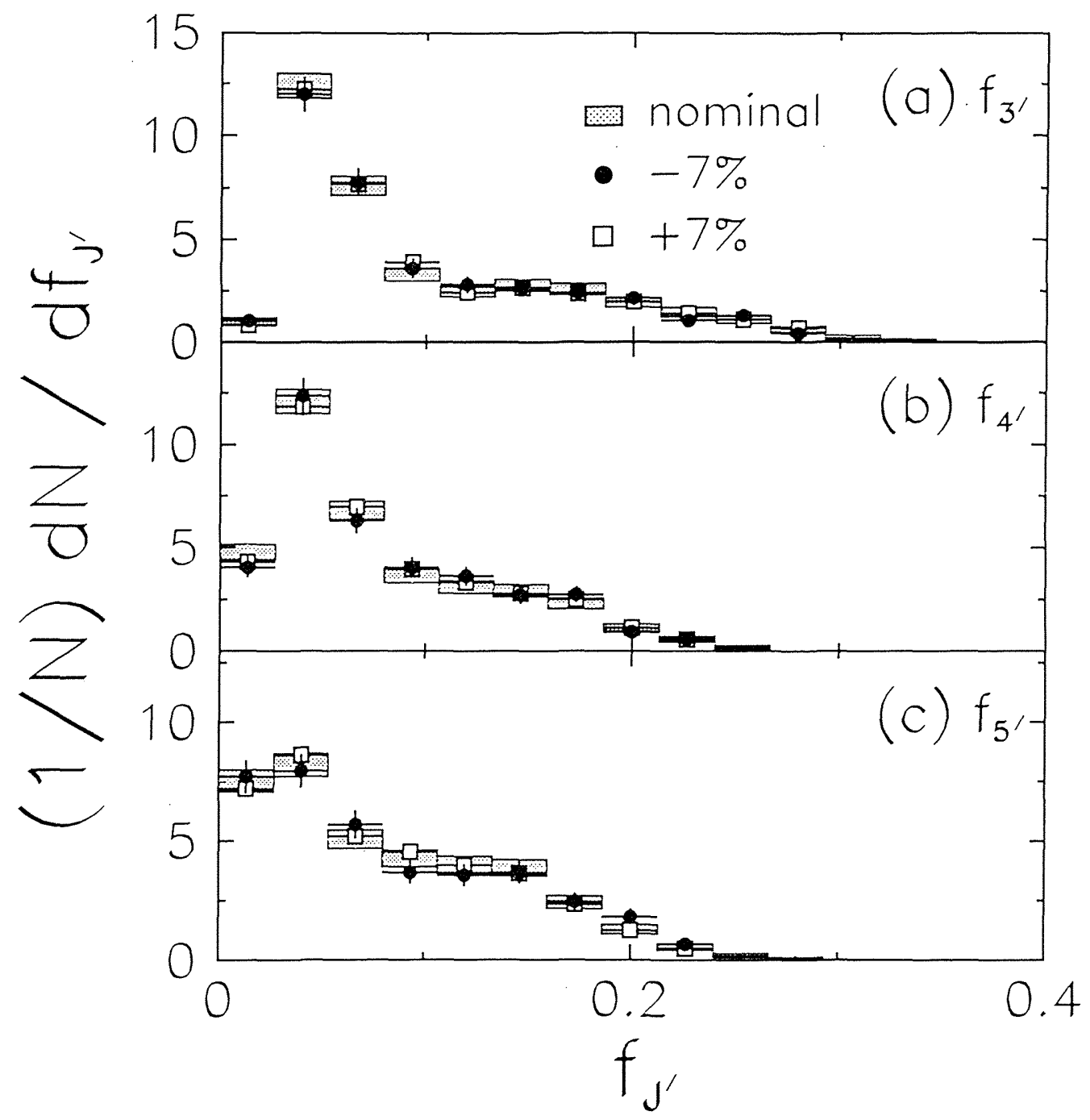

Figure 7.25: The observed $f_{J^{\prime}}\left(J^{\prime}=3^{\prime}, 4^{\prime}, 5^{\prime}\right)$ distributions of 4-jet events for three data samples in which the jet energies were scaled by $-7 \%, 0 \%$, and $+7 \%$. In the figure the histograms correspond to events with the nominal energy scale, points and squares correspond to events with energies shifted by $-7 \%$ and $-7 \%$, respectively. (a) $f_{3^{\prime}}$, (b) $f_{4^{\prime}}$, and $(\mathrm{c}) f_{5^{\prime}}$. 

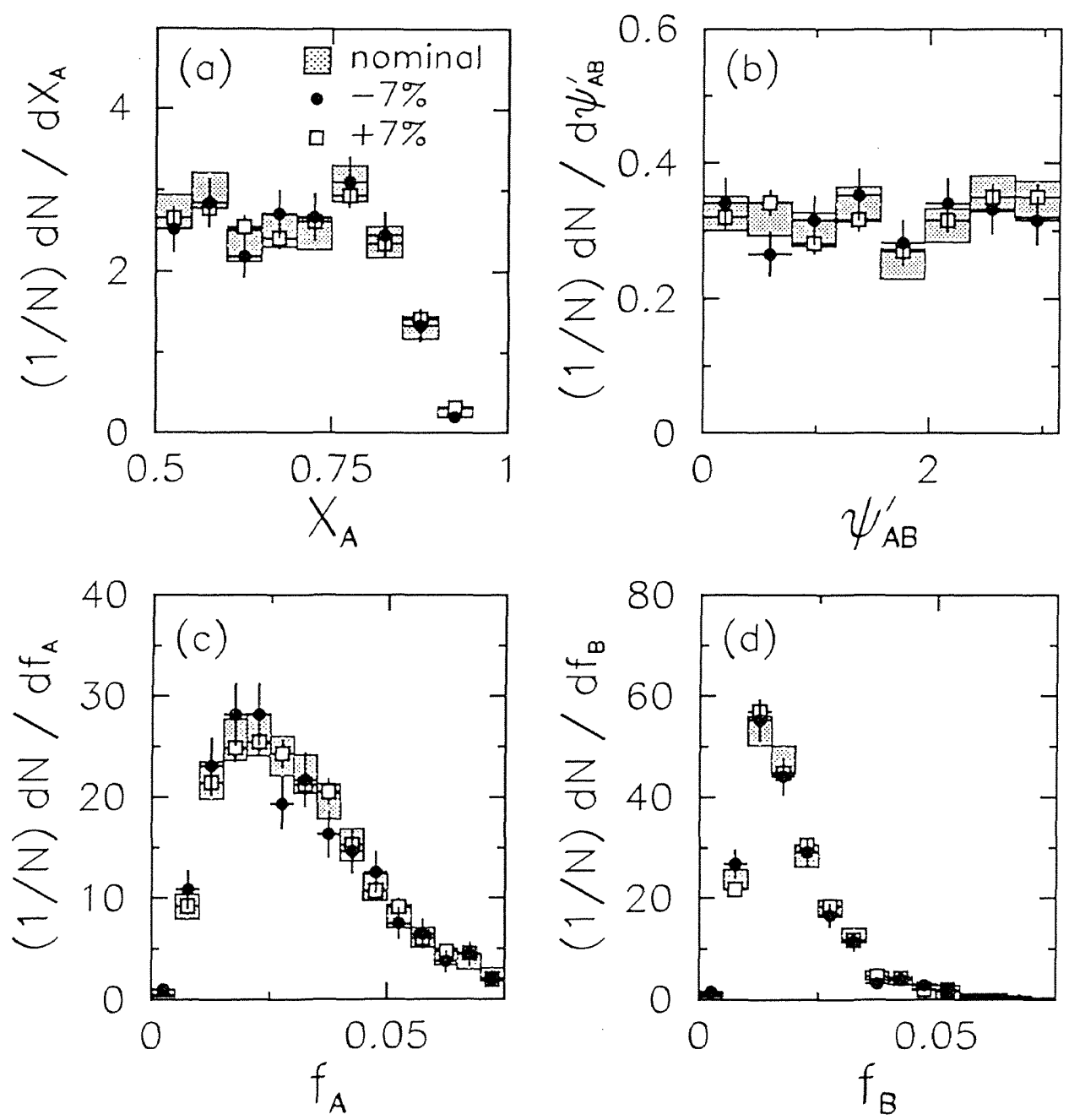

Figure 7.26: The observed two $(A+B)$-body distributions of 4-jet events for three data samples in which the jet energies were scaled by $-7 \%, 0 \%$, and $+7 \%$. In the figure the histograms correspond to events with the nominal energy scale, points and squares correspond to events with energies shifted by $-7 \%$ and $-7 \%$, respectively. (a) $X_{A}$, (b) $\psi_{A B}^{\prime},(\mathrm{c}) f_{A}$, and (d) $f_{B}$. 


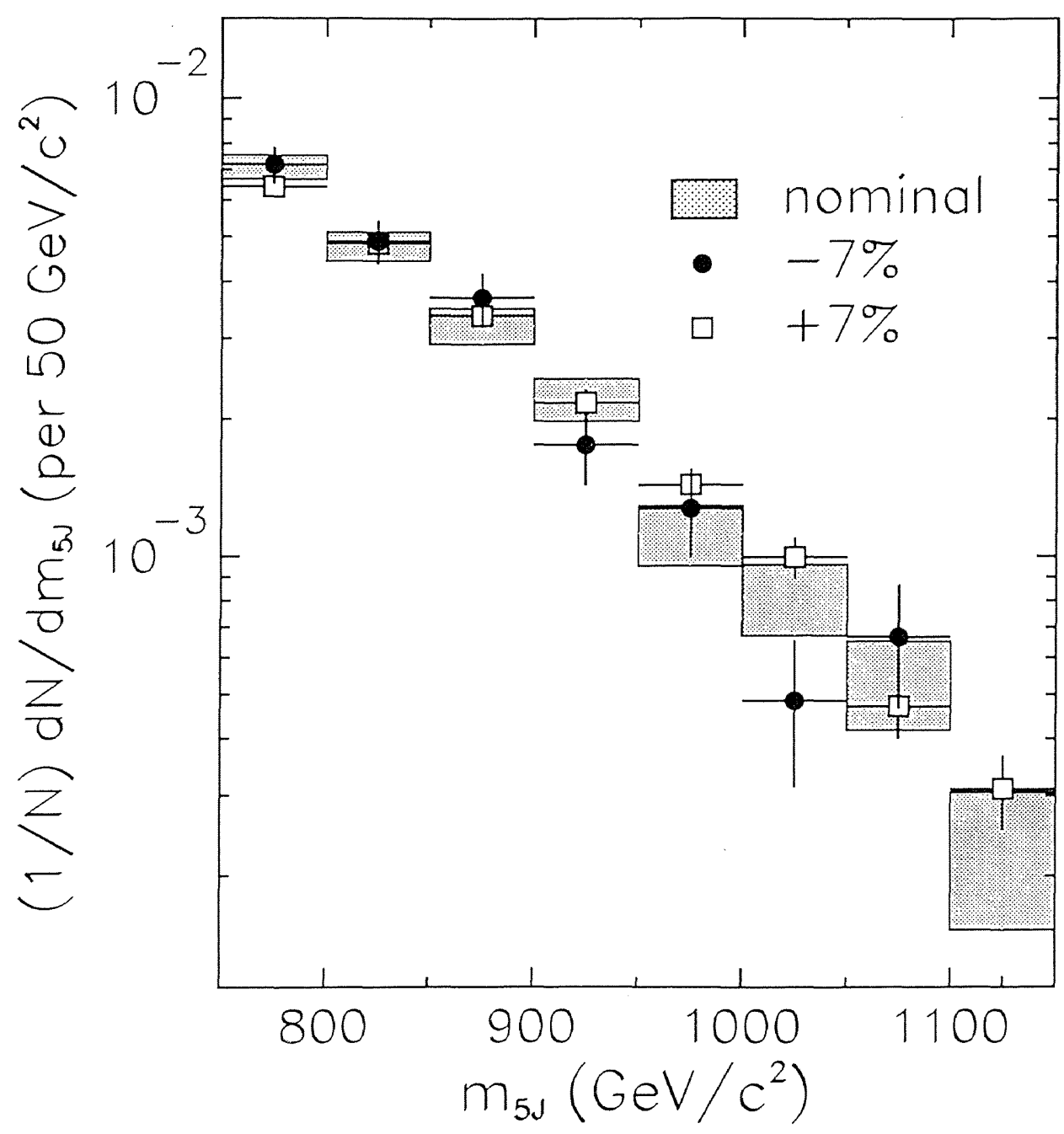

Figure 7.27: The observed $m_{5 J}$ distributions of 5 -jet events for three data samples in which the jet energies were scaled by $-7 \%, 0 \%$, and $+7 \%$. In the figure the histogram corresponds to events with the nominal energy scale, points and squares correspond to events with energies shifted by $-7 \%$ and $-7 \%$, respectively. 

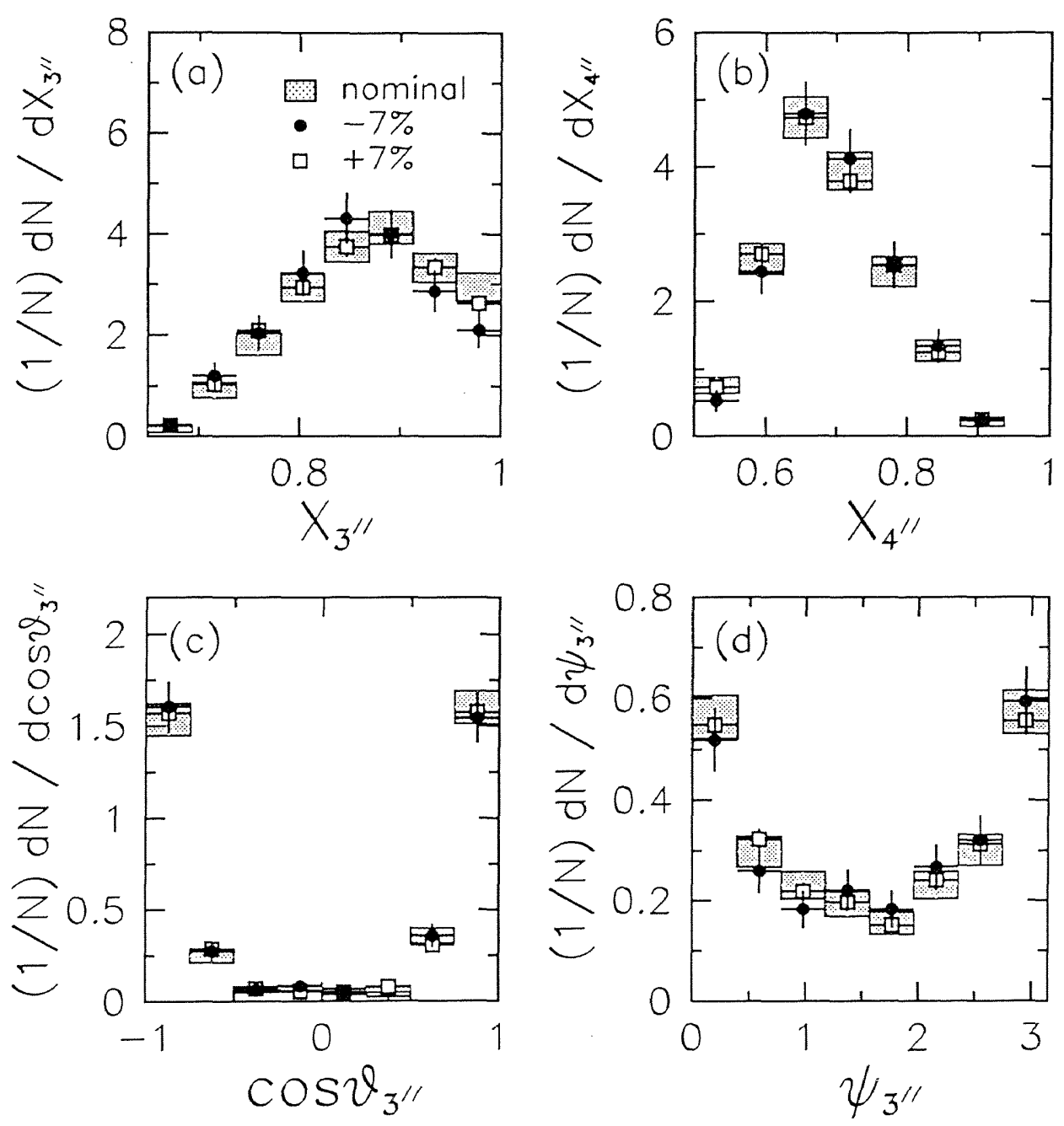

Figure 7.28: The observed three-body distributions of 5-jet events for three data samples in which the jet energies were scaled by $-7 \%, 0 \%$, and $+7 \%$. In the figure the histograms correspond to events with the nominal energy scale, points and squares correspond to events with energies shifted by $-7 \%$ and $-7 \%$, respectively. (a) $X_{3^{\prime \prime}}$, (b) $X_{4^{\prime \prime}},(\mathrm{c}) \cos \theta_{3^{\prime \prime}}$, and (d) $\psi_{3^{\prime \prime}}$. 


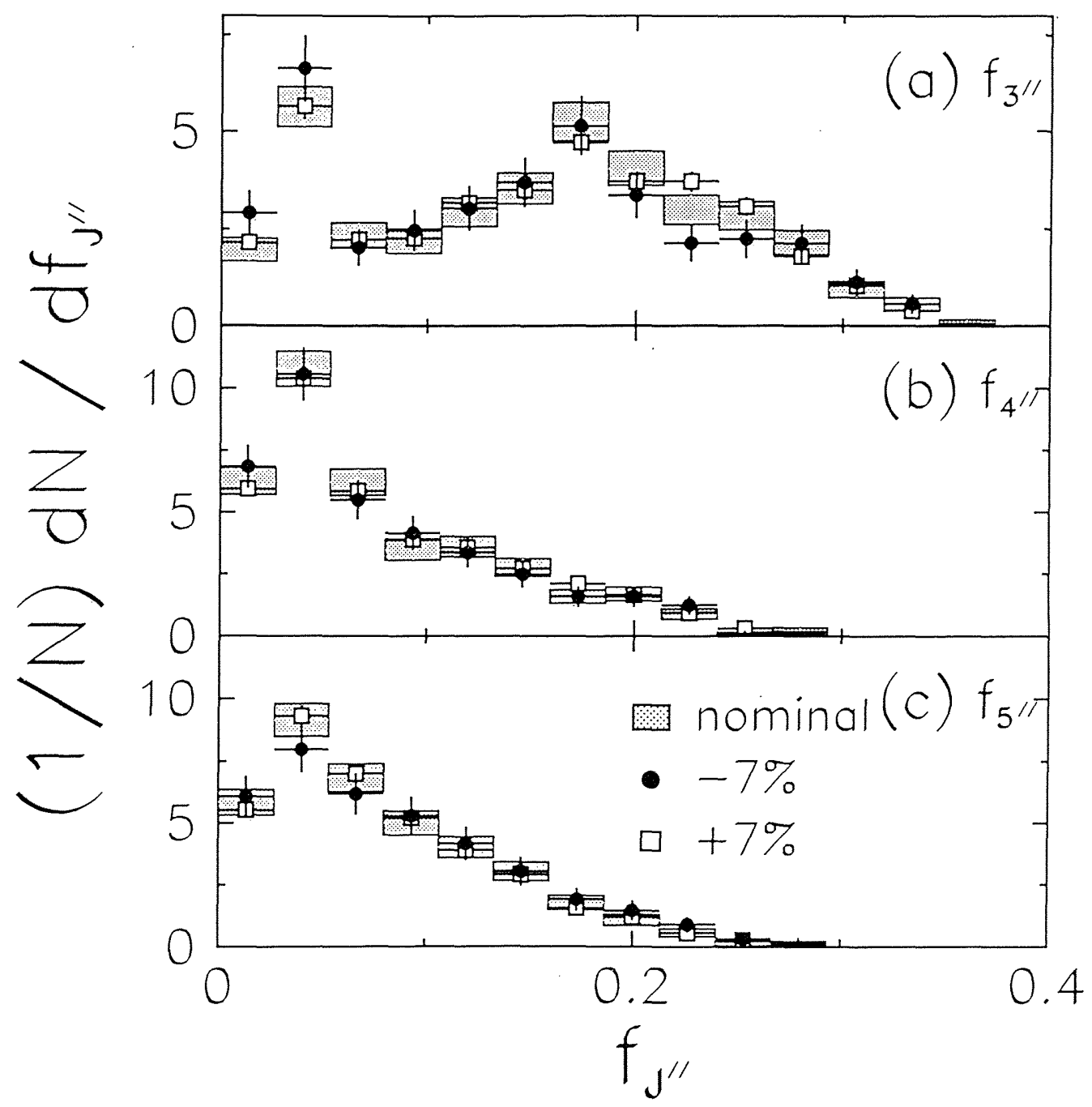

Figure 7.29: The observed $f_{J^{\prime \prime}}\left(J^{\prime \prime}=3^{\prime \prime}, 4^{\prime \prime}, 5^{\prime \prime}\right)$ distributions of 5-jet events for three data samples in which the jet energies were scaled by $-7 \%, 0 \%$, and $+7 \%$. In the figure the histograms correspond to events with the nominal energy scale, points and squares correspond to events with energies shifted by $-7 \%$ and $-7 \%$, respectively. (a) $f_{3^{\prime \prime}},(\mathrm{b}) f_{4^{\prime \prime}}$, and $(\mathrm{c}) f_{5^{\prime \prime}}$ 

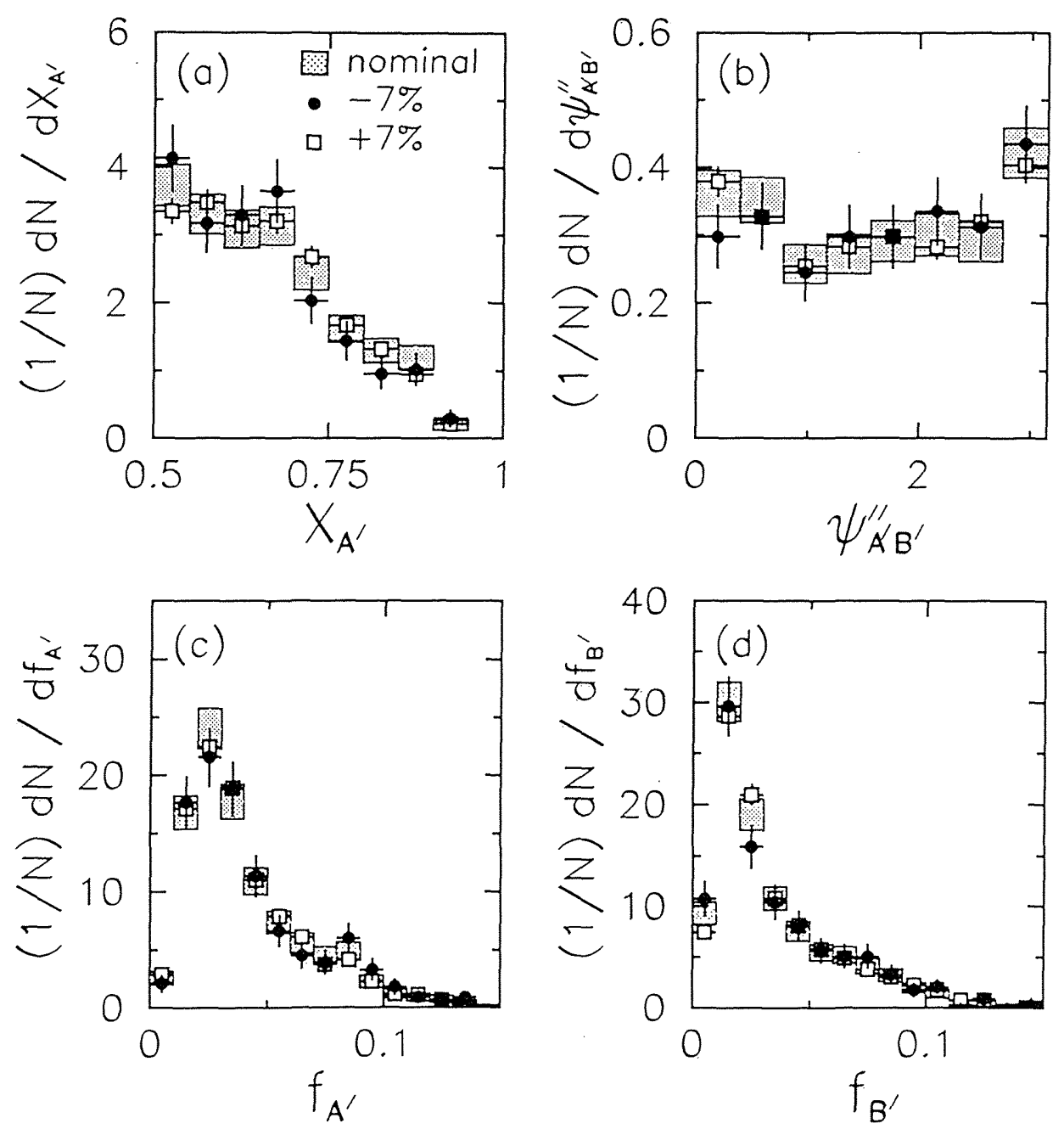

Figure 7.30: The observed two $\left(A^{\prime}+B^{\prime}\right)$-body distributions of 5-jet events for three data samples in which the jet energies were scaled by $-7 \%, 0 \%$, and $+7 \%$. In the figure the histograms correspond to events with the nominal energy scale, points and squares correspond to events with energies shifted by $-7 \%$ and $-7 \%$, respectively. (a) $X_{A^{\prime}}$, (b) $\psi_{A^{\prime} B^{\prime}}^{\prime \prime},\left(\right.$ c) $f_{A^{\prime}}$, and (d) $f_{B^{\prime}}$. 

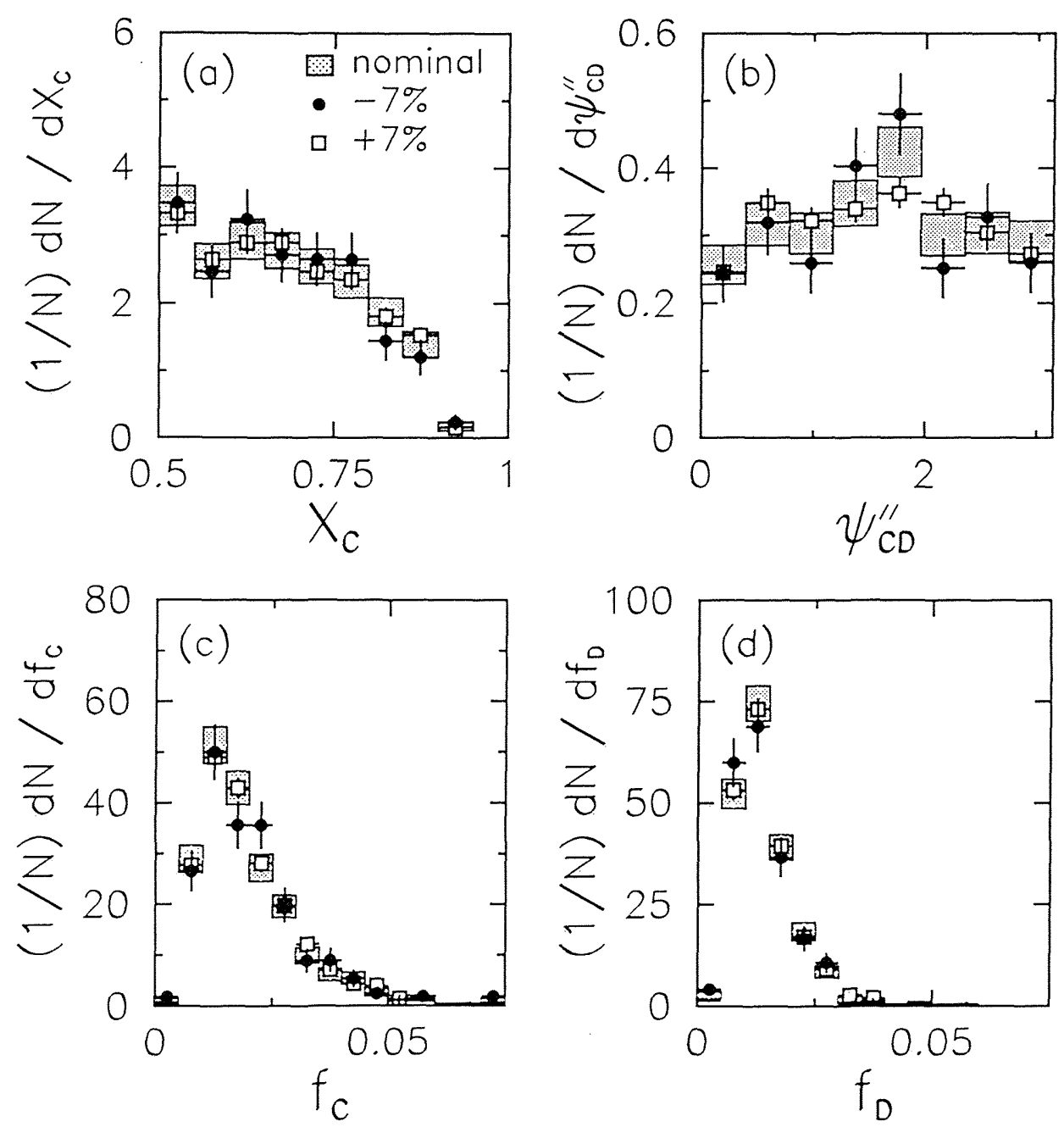

Figure 7.31: The observed two $(C+D)$-body distributions of 5-jet events for three data samples in which the jet energies were scaled by $-7 \%, 0 \%$, and $+7 \%$. In the figure the histograms correspond to events with the nominal energy scale, points and squares correspond to events with energies shifted by $-7 \%$ and $-7 \%$, respectively. (a) $X_{C}$, (b) $\psi_{C D}^{\prime \prime},(\mathrm{c}) f_{C}$, and (d) $f_{D}$. 


\section{Chapter 8}

\section{Conclusions}

The properties of high-mass three-jet, four-jet, and five-jet events produced at the Fermilab Tevatron proton-antiproton collider have been compared with NJETS LO QCD matrix element predictions, HERWIG QCD parton shower Monte Carlo predictions, and predictions from a model in which events are distributed uniformly over the available multibody phase-space. We conclude that the LO QCD predictions give a reasonable first description of the observed three-jet, four-jet, and five-jet multijet distributions that correspond to a set of variables that span the full multijet parameter space. In contrast, the phase-space model is unable to describe the shapes of multijet distributions in regions of parameter space where the QCD calculations predict large contributions from initial- and final-state gluon radiation. We do not see clear evidence for any deviation from the predicted multijet distributions that might indicate new phenomena associated with the presence of many hard partons in the final state. The agreement between data, NJETS, and HERWIG suggests that $2 \rightarrow 2$ scattering plus gluon radiation provides a good approximation to the full LO QCD matrix element for events with three, four, or even five jets in the final state. 


\section{Bibliography}

[1] F. Abe et al. (CDF Collaboration), Phys. Rev. Lett. 75, 608 (1995).

[2] F. A. Berends et al., Phys. Lett. B 118, 124 (1981); Z. Kunst and E. Pietarinen, Nucl. Phys. B 164, 45 (1980); T. Gottschalk and D. Sivers, Phys. Rev. D 21, 102 (1980).

[3] J. Gunion and Z. Kunst, Phys. Lett. B 159, 167 (1985); S. Parke and T. Taylor, Nucl. Phys. B 269, 410 (1986); Z. Kunst, Nucl. Phys. B 271, 333 (1986); J. Gunion and Z. Kunst, Phys. Lett. B 176, 163 (1986); J. Gunion and J. Kalinowski, Phys. Rev. D 34, 2119 (1986); S. Parke and T. Taylor, Phys. Rev. D 35, 313 (1987); F. A. Berends and W. T. Giele, Nucl. Phys. B 294, 700 (1987); M. Mangano, S. Parke, and Z. Xu, Nucl. Phys. B 298, 673 (1988); M. Mangano and S. Parke, Nucl. Phys. B 299, 190 (1987).

[4] F.A. Berends, W. Giele, and H. Kuijf, Nucl. Phys. B 333, 120 (1990); Phys. Lett. B 232, 266 (1990); F.A. Berends and H. Kuijf, Nucl. Phys. B 353, 59 (1991).

[5] F. A. Berends, W. T. Giele, and H. Kuijf, Phys. Lett. B 232, 266 (1989).

[6] F. A. Berends and W. T. Giele, Nucl. Phys. B 306, 759 (1988).

[7] Z. Kunst and W. J. Stirling, Phys. Lett. B 171, 307 (1986); Phys. Rev. D 56, 2493 (1988); C. J. Maxwell, Phys. Lett. B 192, 190 (1987); Nucl. Phys. B 316, 321 (1989); S. J. Parke and T. R. Taylor, Phys. Rev. Lett. 56, 2459 (1986); M. L. Mangano and S. J. Parke, Phys. Rev. D 39, 758 (1989); C. J. Maxwell and S. J. Parke, Phys. Rev. D 44, 2727 (1991). 
[8] G. Arnison et al. (UA1 Collaboration), Phys. Lett. B 123, 115 (1983); B 132, 214 (1983); B 136, 294 (1984); B 177, 244 (1986); C. Albajar et al. (UA1 Collaboration), Phys. Lett. B 209, 127 (1988).

[9] G. Arnison et al. (UA1 Collaboration), Phys. Lett. B 158, 494 (1985).

[10] A Study of Two-Jet and Three-Jet Production at the CERN Proton-Antiproton Collider, Elizabeth Buckley (Thesis), Dept. of Physics, Queen Mary College, University of London (1986).

[11] M. Banner et al. (UA2 Collaboration), Phys. Lett. B 118, 203 (1982); P. Bagnaia et al. (UA2 Collaboration), Phys. Lett. B 138, 430 (1984); B 144, 283 (1984); J. A. Appel et al. (UA2 Collaboration), Phys. Lett. B 165, 441 (1985); J. Alitti et al. (UA2 Collaboration), Z. Phys. C 49, 17 (1991).

[12] J. A. Appel et al. (UA2 Collaboration), Z. Phys. C 30, 341 (1986).

[13] F. Abe et al. (CDF Collaboration), Phys. Rev. Lett. 62, 3020 (1989); 64, 157 (1990); 69, 2896 (1992); 71, 2542 (1993); Phys. Rev. D 41, 1722 (1990); D 48, 998 (1993).

[14] F. Abe et al. (CDF Collaboration), Phys. Rev. D 45, 1448 (1992).

[15] S. Abachi et al. (D0 Collaboration), FERMILAB Conf-95/214-E, submitted to the Int. Conf. on High Energy Physics, Brussels, Belgium, July 27 - Aug. 2 (1995).

[16] J. Alitti et al. (UA2 Collaboration), Phys. Lett. B 268, 145 (1991).

[17] F. Abe et al. (CDF Collaboration), Phys. Rev. D 47, 4857 (1993).

[18] F. Abe et al. (CDF Collaboration), Nucl. Instrum. Methods A 271, 387 (1988).

[19] G. Arnison et al. (UA1 Collaboration), Phys. Lett. B 132, 214 (1983).

[20] J. Alitti et al., CERN Report No. CERN-PPE/90-105 (1990).

[21] F. Abe et al. (CDF Collaboration), Phys. Rev. D 45, 2249 (1992). 
[22] G. Marchesini and B. Webber, Nucl. Phys. B 310, 461 (1988).

[23] H.L. Lai et al., Preprint MSU-HEP-41024, CTEQ-404, (to be published).

[24] A.D. Martin, R.G. Roberts, W.J. Stirling; Phys. Lett. B 306, 145 (1993).

[25] CERN Program Library Manual 1989.10.03, Routine W515, p. 6.503.

[26] S. Geer and T. Asakawa, FERMILAB-PUB-95/333, to be published in Phys. Rev. D.

[27] J. Collins and D. Soper, Phys. Rev. D 16, 2219 (1977).

[28] R. H. Dalitz, Phyl. Mag. 44, 1068 (1953); E. Fabri, Nuovo Cimento. 11, 479 (1954). 


\section{Appendix A}

\section{Event Pictures}

In the analysis described in this thesis the jet $E_{T}$ requirement $\left(E_{T}>20 \mathrm{GeV}\right)$ is soft compared with the $\sum E_{T}$ requirement $\left(\sum E_{T}>420 \mathrm{GeV}\right)$. Therefore, events in different parts of the Dalitz plane will have very different kinematic configurations. For example, the three-jet events in the sample have topologies that range from "mercedes" type events where all three jets are hard, to events which are essentially two-jet events with a much softer third jet. To illustrate the types of events entering the analysis Figures A.1, A.2, and A.3 show respectively examples of three-jet, four-jet, and five-jet events occupying various parts of the three-body Dalitz plane. In particular, in each figure there is one event from each of the three corners of the kinematically allowed region, and one event from the center of the kinematically allowed region. The event parameters are listed in Tables A.1, A.2, and A.1 


\begin{tabular}{l|rrrr}
\hline \hline Run & 58148 & 63650 & 45753 & 46290 \\
Event & 2443 & 393239 & 238791 & 75308 \\
\hline$\sum E_{T}(\mathrm{GeV})$ & 534 & 542 & 467 & 507 \\
$m_{3 J}(\mathrm{GeV})$ & 712 & 603 & 651 & 627 \\
$X_{3}$ & 0.89 & 0.89 & 0.68 & 0.85 \\
$X_{4}$ & 0.89 & 0.57 & 0.66 & 0.70 \\
$\cos \theta_{3}$ & 0.57 & 0.26 & 0.02 & 0.50 \\
$\psi_{3}$ & 2.78 & 0.51 & 0.21 & 0.44 \\
$f_{3}$ & 0.04 & 0.06 & 0.06 & 0.05 \\
$f_{4}$ & 0.06 & 0.05 & 0.05 & 0.04 \\
$f_{5}$ & 0.03 & 0.04 & 0.04 & 0.01 \\
\hline \hline
\end{tabular}

Table A.1: Parameters of three-jet events shown in Figure A.1.

\begin{tabular}{l|rrrr}
\hline \hline Run & 58321 & 45776 & 61436 & 66247 \\
Event & 91783 & 239066 & 123216 & 52624 \\
\hline$\sum E_{T}(\mathrm{GeV})$ & 501 & 553 & 434 & 584 \\
$m_{4 J}(\mathrm{GeV})$ & 736 & 663 & 675 & 659 \\
$X_{3^{\prime}}$ & 0.90 & 0.87 & 0.68 & 0.84 \\
$X_{4^{\prime}}$ & 0.88 & 0.57 & 0.67 & 0.71 \\
$\cos \theta_{3^{\prime}}$ & 0.67 & 0.49 & -0.54 & 0.14 \\
$\psi_{3^{\prime}}$ & 2.80 & 0.55 & 3.08 & 2.52 \\
$f_{3^{\prime}}$ & 0.18 & 0.04 & 0.03 & 0.06 \\
$f_{4^{\prime}}$ & 0.10 & 0.04 & 0.16 & 0.03 \\
$f_{5^{\prime}}$ & 0.03 & 0.09 & 0.01 & 0.08 \\
$X_{A}$ & 0.61 & 0.54 & 0.74 & 0.81 \\
$\psi_{A B}^{\prime}$ & 1.17 & 1.53 & 1.57 & 3.03 \\
$f_{A}$ & 0.05 & 0.03 & 0.04 & 0.03 \\
$f_{B}$ & 0.03 & 0.02 & 0.03 & 0.01 \\
\hline \hline
\end{tabular}

Table A.2: Parameters of four-jet events shown in Figure A.2. 


\begin{tabular}{l|rrrr}
\hline \hline Run & 43306 & 46911 & 62867 & 65085 \\
Event & 24330 & 47090 & 10476 & 73048 \\
\hline$\sum E_{T}(\mathrm{GeV})$ & 523 & 627 & 597 & 493 \\
$m_{5 J}(\mathrm{GeV})$ & 920 & 962 & 801 & 878 \\
$X_{3^{\prime \prime}}$ & 0.99 & 0.99 & 0.71 & 0.87 \\
$X_{4^{\prime \prime}}$ & 0.90 & 0.55 & 0.67 & 0.70 \\
$\cos \theta_{3^{\prime \prime}}$ & -0.92 & -0.70 & -0.08 & -0.98 \\
$\psi_{3^{\prime \prime}}$ & 2.28 & 2.98 & 2.63 & 2.88 \\
$f_{3^{\prime \prime}}$ & 0.17 & 0.28 & 0.22 & 0.17 \\
$f_{4^{\prime \prime}}$ & 0.04 & 0.12 & 0.05 & 0.07 \\
$f_{5^{\prime \prime}}$ & 0.01 & 0.02 & 0.04 & 0.03 \\
$X_{A^{\prime}}$ & 0.51 & 0.60 & 0.51 & 0.51 \\
$\psi_{A^{\prime} B^{\prime}}^{\prime \prime}$ & 0.19 & 0.69 & 1.80 & 1.87 \\
$f_{A^{\prime}}$ & 0.06 & 0.04 & 0.10 & 0.09 \\
$f_{B^{\prime}}$ & 0.04 & 0.06 & 0.03 & 0.01 \\
$X_{C}$ & 0.74 & 0.85 & 0.73 & 0.64 \\
$\psi_{C D}^{\prime \prime}$ & 0.87 & 2.53 & 2.41 & 1.00 \\
$f_{C}$ & 0.01 & 0.04 & 0.03 & 0.01 \\
$f_{D}$ & 0.01 & 0.01 & 0.02 & 0.01 \\
\hline \hline
\end{tabular}

Table A.3: Parameters of five-jet events shown in Figure A.3. 


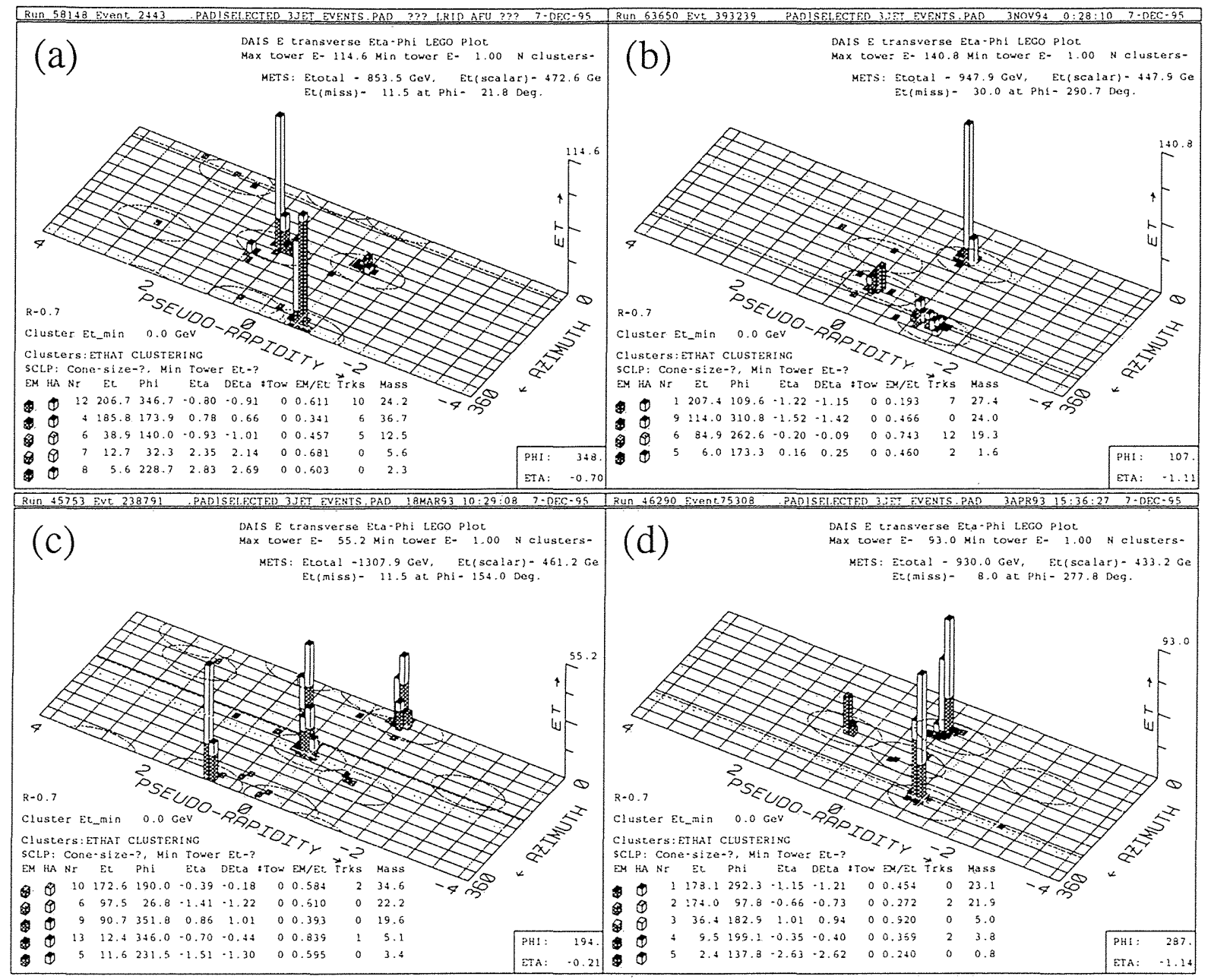

Figure A.1: Event Pictures for events with 3 Jets and (a) $X_{3}=0.89$ and $X_{4}=0.89$, (b) $X_{3}=0.89$ and $X_{4}=0.57$, (c) $X_{3}=0.68$ and $X_{4}=0.66$, and (d) $X_{3}=0.85$ and $X_{4}=0.70$. 


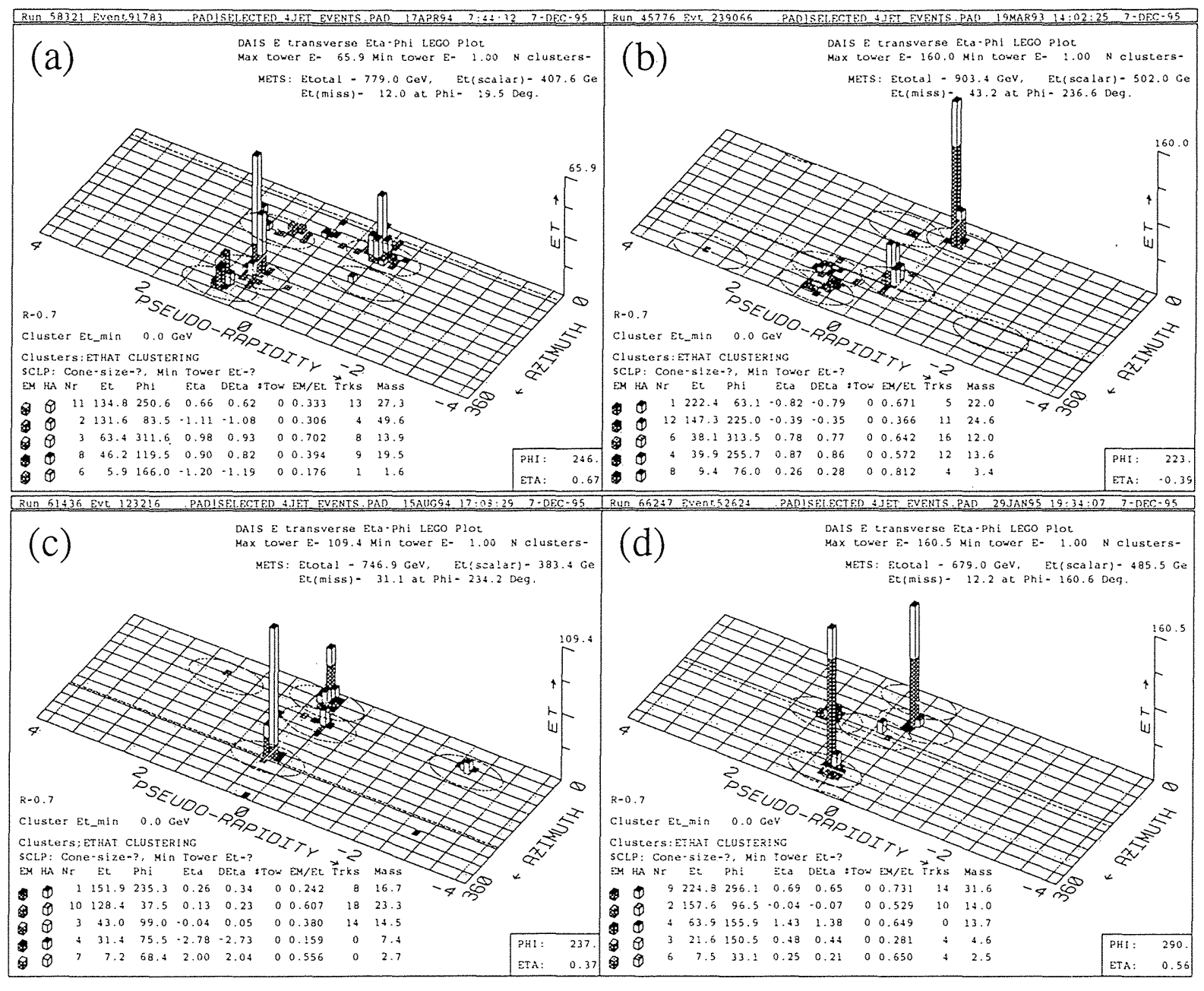

Figure A.2: Event Pictures for events with 4 Jets and (a) $X_{3^{\prime}}=0.90$ and $X_{4^{\prime}}=0.88$, (b) $X_{3^{\prime}}=0.87$ and $X_{4^{\prime}}=0.57$, (c) $X_{3^{\prime}}=0.68$ and $X_{4^{\prime}}=0.67$, and (d) $X_{3^{\prime}}=0.84$ and $X_{4^{\prime}}=0.71$. 


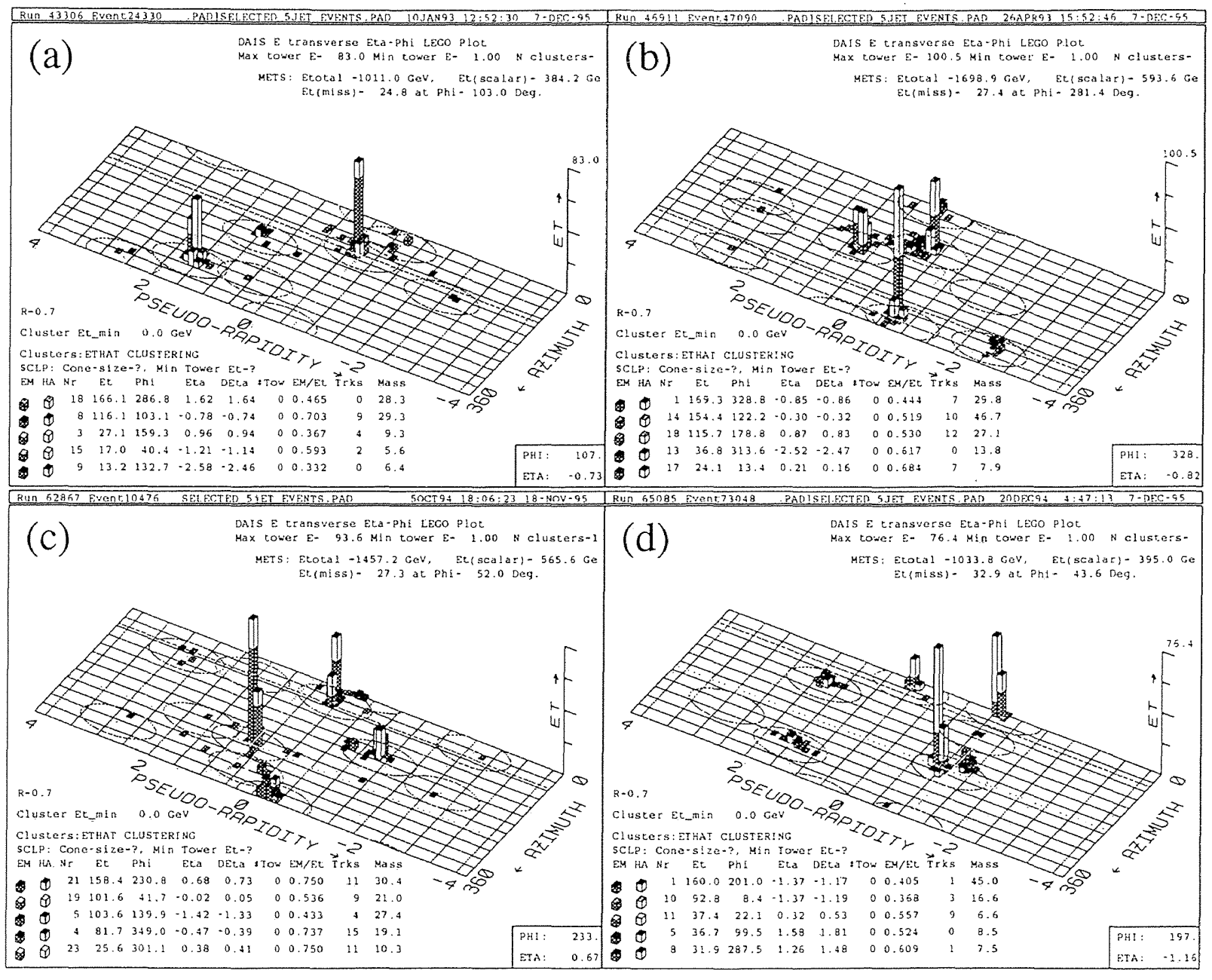

Figure A.3: Event Pictures for events with 5 Jets and (a) $X_{3^{\prime \prime}}=0.99$ and $X_{4^{\prime \prime}}=0.90$, (b) $X_{3^{\prime \prime}}=0.99$ and $X_{4^{\prime \prime}}=0.55$, (c) $X_{3^{\prime \prime}}=0.71$ and $X_{4^{\prime \prime}}=0.67$, and (d) $X_{3^{\prime \prime}}=0.87$ and $X_{4^{\prime \prime}}=0.70$. 


\section{Appendix B}

\section{Validity of our $\chi^{2}$ test}

We have checked the validity of our $\chi^{2}$ test by a Monte Carlo technique. We generated M.C. samples of a Gaussian distribution and histogramed in 5 bins. Then we calculated $\chi^{2}$ 's by comparing the first 1000 histograms with the Gaussian distribution which is given by a function and was used to generate the histograms. The definition of $\chi^{2}$ is:

$$
\chi_{1}^{2} \equiv \sum \frac{\left(y_{1}(i)-G\left(x_{i}\right)\right)^{2}}{\sigma_{y_{1}}(i)^{2}},
$$

where $y_{1}(i)$ is the content of a generated histogram in the $i$-th bin, $\sigma_{y_{1}}(i)$ is the statistical error in the bin, and $G\left(x_{i}\right)$ is the Gaussian function. This comparison results in a set of $1000 \chi^{2}$ 's. We made another $\chi^{2}$ distribution by comparing the first 1000 histograms with the second 1000 histograms. All bins have statistical errors and the definition of $\chi^{2}$ is:

$$
\chi_{2}^{2} \equiv \sum \frac{\left(y_{1}(i)-y_{2}(i)\right)^{2}}{\sigma_{y_{1}}(i)^{2}+\sigma_{y_{2}}(i)^{2}}
$$

This form is exactly the same as one used in our analysis. Finally we compared the $\chi_{2}^{2}$ distribution with the $\chi_{1}^{2}$ distribution and the $\chi_{\exp }^{2}$ distribution statistically expected for N.D.F $=4$. (Note: Number of degrees of freedom is given by number of bins $\mathrm{min}$ See Figure B.1-(a). In the figure the $\chi_{2}^{2}$ distribution (points) are compared with the $\chi_{1}^{2}$ distribution (histogram) and the theoretical $\chi_{\exp }^{2}$ distribution (curve). They are seen to be in a good agreement with each other. Figures B.1-(b), -(c), and -(d) are distributions for numbers of bins of 10,20 , and 40 respectively. These results suggest that our method used to estimate the levels of agreements is reasonable. 

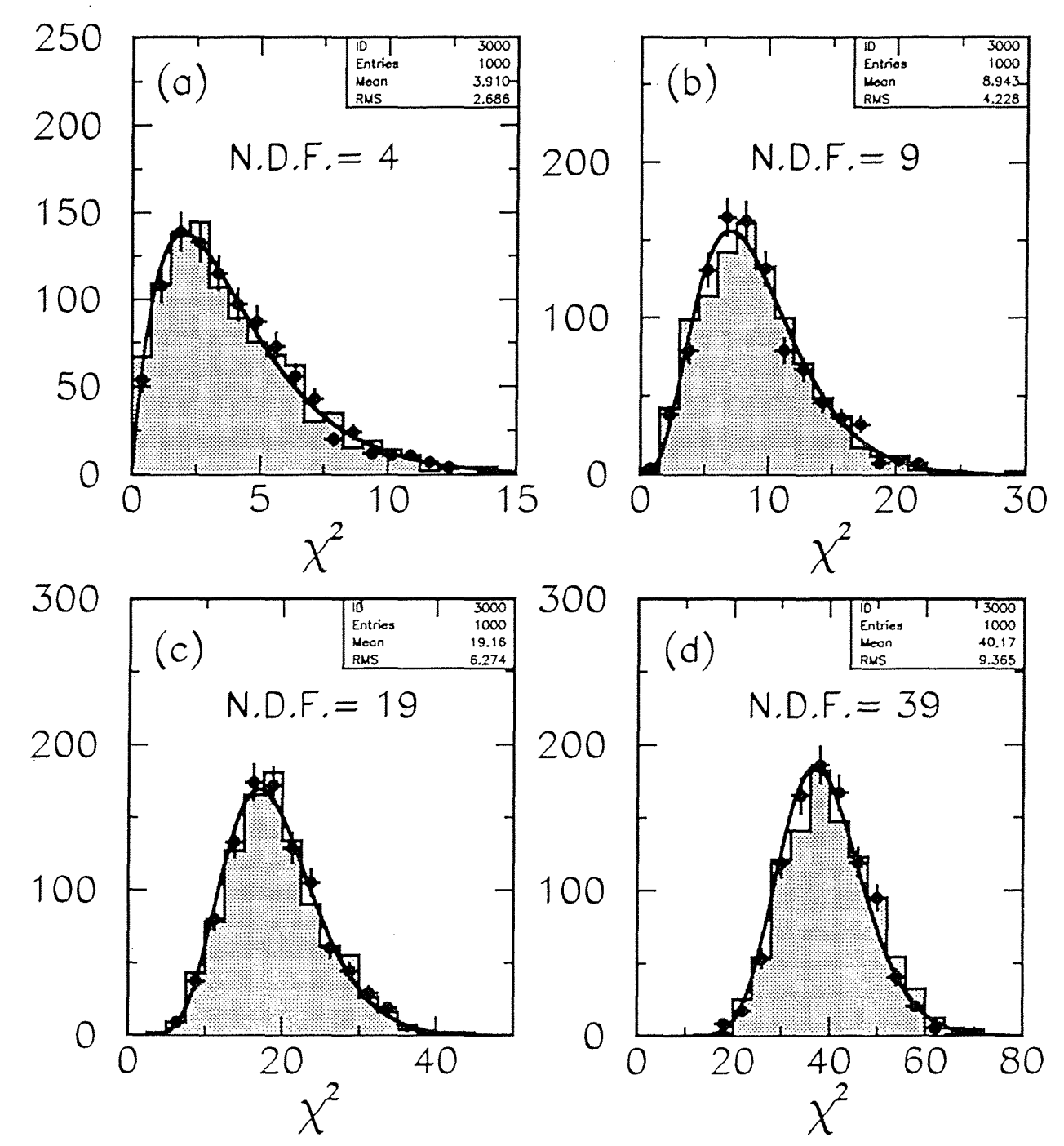

Figure B.1: The $\chi^{2}$ distributions. $\chi_{2}^{2}$ (points) compared with $\chi_{1}^{2}$ (histogram) and $\chi_{\exp }^{2}$ (curve). (a), (b), (c) and (d) are for the number of bins $=5,10,20$, and 40, respectively. 


\section{Appendix C}

\section{Analytic Form of $X$ Variable}

To gain some insight into the shape of the phase-space model prediction for the distribution of $X_{A}$, which was defined as a four-jet variable in Chapter 4, consider a system of four-massless particles labeled randomly 1,2,3 and 4. The four-body phase-space $\Phi_{4}$ is given by:

$$
\begin{aligned}
\Phi_{4} & =\int \frac{d p_{1}^{3}}{2 E_{1}} \frac{d p_{2}^{3}}{2 E_{2}} \frac{d p_{3}^{3}}{2 E_{3}} \frac{d p_{4}^{3}}{2 E_{4}} \delta^{(4)}(E, p) \\
& =\frac{1}{16} \int \frac{d p_{1}^{3} d p_{2}^{3} d p_{3}^{3}}{E_{1} E_{2} E_{3} E_{4}} \delta(E)
\end{aligned}
$$

where $E_{i}$ and $p_{i}$ are the energy and the momentum of particle $i$, and $\delta$ function represents the four-momentum conservation. (C.2) can be written with $p_{12}$ which is the absolute value of the momentum of $1+2$ system, and $\phi$ which is the azimuthal angel of $\overrightarrow{p_{3}}$ with $\overrightarrow{p_{1}}+\overrightarrow{p_{2}}$ as follows:

$$
\begin{aligned}
\Phi_{4} & =\frac{\pi^{2}}{2} \int d E_{1} d E_{2} d E_{3} d P_{12} d \phi \\
& \sim \int d E_{1} d E_{2} d E_{3} d p_{12}
\end{aligned}
$$

We define a variable $\mathrm{X}$ such that:

$$
X \equiv \frac{E_{1}}{E_{1}+E_{2}} \text { for } E_{1}>E_{2}
$$

Using $X,(C .4)$ is written as follows:

$$
\Phi_{4} \sim \int \frac{1}{(1-X)^{2}} E_{2} d E_{3} d p_{12} d X
$$


The integrations with all of the variables except for $X$ provides the shape of the $X$ distribution as follows:

$$
\frac{d \Phi_{4}}{d X} \sim-\frac{1}{X^{3}}+\frac{3}{X^{2}}-2
$$

